\title{
Colorimetric Aptasensors for the Detection of Methamphetamine in Saliva
}

A thesis submitted to the Victoria University of Wellington in fulfilment of the requirements for the degree of Doctor of Philosophy in Chemistry

$$
\text { by }
$$

Anindita Sen

Supervised by Prof. Justin M. Hodgkiss

Victoria University of Wellington 



\section{Abstract}

There has been a long-standing need and an increasing demand for a low cost, sensitive, selective, simple, and portable detector (semi quantitative/quantitative) for improved screening of drugs of abuse. Methamphetamine (meth) was chosen as the primary drug to detect due to its obvious prevalence as the most abused substance in New Zealand. Impairment caused by meth use while driving and at workplaces are a cause of serious concern which needs immediate attention of scientists so that rapid and reliable on-site applicable quantitative tests can be developed to address the growing concern. Currently, urine drug tests are most common, however these tests suffer from concerns relating to privacy invasion, or chances of adulteration when privacy is granted to the suspect. In order to overcome these issues, saliva is emerging as an alternative and promising matrix for meth detection.

In this context, this project aims to develop a non-invasive assay that produces a colourimetric response in the presence of meth in human saliva. The assay features coloured gold nanoparticles (AuNPs) coated with synthetic DNA aptamers acting as the receptor for methamphetamine. Aptamers are seen as promising for biosensing applications owing to the ease of synthetically evolving nucleotide sequences to specifically bind a range of targets, along with their high stability and ease of synthesis. This colorimetric aptasensor assay not only allows the visual determination of analyte in the sample by naked eye, but simultaneously a quantitative interpretation is possible by UV/Vis spectroscopy.

Using an aptamer selected to bind to meth, we first optimized the AuNPs based colorimetric assay in buffer. In this assay, colourimetric signals are generated when target binding induces aptamer dissociation from AuNP surfaces, causing them to aggregate and change colour. Whereas this assay assumes an equilibrium competition between aptamers binding to AuNPs versus the target, we discovered a pronounced time dependence in the aptamer-AuNP interactions. Over time, the aptamers became more strongly bound to the AuNPs and less available to interact with the target. This knowledge led us to demonstrate an assay with a modified sequence of steps to mitigate this problem, enabling successful detection of meth in buffer below the legal cut-off level for saliva $25 \mathrm{ng} / \mathrm{mL}(\sim 167 \mathrm{nM})$.

Our new knowledge of aptamer-AuNP interactions also suggested that traditional aptamer selection strategies are not ideal for subsequent application in colourimetric sensors. Therefore, 
we obtained a new meth binding aptamer that was selected via a novel method based on its association/dissociation with AuNPs in the presence of target. Indeed, we found that colourimetric sensors using the aptamer selected in this way did not suffer from the problems identified above and ultimately exhibited better sensing performance.

Following this, a demonstration of meth detection in saliva was also achieved at levels of detection approaching the legal cut-off level. As well as comparing the response in saliva versus buffer and different testing protocols, successful blind tests were also conducted with unknown saliva samples. Extensive new knowledge about the nature of interactions between AuNPs and the salivary matrix was gained to underpin these sensing demonstrations, and this knowledge also highlights further challenges to overcome before these sensors can be applied outside of the lab.

To facilitate the translation of the assay into an on-site applicable system, it is necessary to look for simple detection systems that are suitable for roadside or workplace applications and reliable and sensitive enough to replace the standard UV-Vis spectrophotometer for quantifying the assay. This motivated the prototype concept of a cost-effective, sensitive, and reliable single channel absorption based light meter device integrated with a sample holder and LED. The promising detection levels achieved ( $63 \mathrm{nM}$ or $9.4 \mathrm{ng} / \mathrm{mL})$ with the device costing less than $\$ 100$ showed immense scope for the future development of a simple on-site applicable quantitative drug detection device.

The knowledge created in this work provide a clear picture about the challenges and opportunities in the detection of drugs and other small molecules. Provided that aptamers are selected with consideration for the downstream sensing mechanism, sensitive, robust, and simple sensing assays are close to reality for real samples such as saliva. 


\section{Acknowledgements}

The journey of $\mathrm{PhD}$ has been unique and tremendously humbling in terms of both personal and scientific developement. As I complete this PhD journey, I would like to acknowledge certain people who have been instrumental in the successful completion of my $\mathrm{PhD}$.

Firstly, I would like to thank my supervisor Professor Justin Hodgkiss whose support and guidance has been priceless in guiding me through the difficult times in my $\mathrm{PhD}$ journey. Immense gratitude to him for giving me the opportunity to research on such a relevant and interesting topic and encouraging me to develop my scientific acumen.

My sincere gratitude to the 'Hodgkiss group' for providing a friendly environment throughout my journey. I would especially like to mention Clément and Yasmin for their supportive and motivating discussions to help me keep my spirits up during this journey.

I would like to thank Professor Joanne Harvey and Paul for giving me useful feedback on my manuscripts and especially Paul for clarifying my conceptual understanding on several topics.

I would like to acknowledge the contribution of Karen in helping to speed up the process of designing the light meter device. Special thanks to Manu from SCPS workshop for prioritizing the building of the light meter device on such short notice.

I would like to acknowledge the useful discussions with my collaborators from ESR and for providing saliva samples for my research. I am grateful to my University for granting me access to my office space which helped me write up my thesis without delays during an ongoing pandemic situation.

I would like to acknowledge the MBIE (Ministry of Business Innovation and Employment) scholarship for funding my research and living costs in New Zealand.

Special thanks to my family for always being there for me, believing in me and motivating me to move forward each day. Most importantly, I would like to thank my best friend and husband, Rishabh for supporting me through my highs and lows during this journey like a rock. Without your support, it would have been hard to complete this journey! 


\section{Research outputs}

\section{Expected journal articles}

1) "Accounting for interaction kinetics between gold nanoparticles and aptamers enables high performance colorimetric sensors" Sen A, Poulsen H, Sester C, Hodgkiss J. (manuscript submitted to Analytical Chemistry)

2) "The nature of interferences when applying colorimetric aptasensors in human saliva" Sen A, Poulsen H, Hodgkiss J. (manuscript in preparation for submission)

3) "Fabrication and validation of a cost-effective and sensitive handheld device for methamphetamine detection" Sen A, Hodgkiss J (manuscript in preparation)

\section{Conference posters and oral presentations}

1) Selected for attending and presenting a poster on 'Aptasensors for the detection of methamphetamine' in the NIMS-MANA international winter workshop Japan. 2018

2) Runner-up position secured in '3-minute thesis' competition held at Victoria University of Wellington yearly. 2018

3) 3-minute introductory talk and poster presentation titled 'Colorimetric Biosensors for the detection of Methamphetamine in oral fluid' in an international conference AMN9 held in Wellington, NZ. 2019

4) Oral presentation titled 'Accounting for the interaction kinetics between gold nanoparticles and aptamers enables high performance colorimetric sensors' at the $15^{\text {th }}$ international conference and exhibition on Biosensors and Bioelectronics October 2223, Montreal, Canada 2021. 


\section{Abbreviations and common terms}

\begin{tabular}{|c|c|}
\hline Aptamer & DNA oligonucleotides that bind to targets specifically \\
\hline Aptasensor & Aptamer based biosensor \\
\hline AuNPs & Gold nanoparticles \\
\hline BWB & Binding and washing buffer \\
\hline cDNA & Complimentary DNA \\
\hline DNA & Deoxyribonucleic acid \\
\hline ds DNA & Double stranded DNA \\
\hline ITC & Isothermal titration calorimetry \\
\hline LOD & Limit of Detection \\
\hline LSPR & Localized Surface Plasmon Resonance \\
\hline MDA & 3,4-Methylenedioxyamphetamine \\
\hline MDMA & Methylenedioxymethamphetamine \\
\hline Meth & Methamphetamine \\
\hline POC & Point-of-Care \\
\hline SELEX & Systematic evolution of ligands by exponential enrichment \\
\hline SG & SYBR Green I dye \\
\hline SPR & Surface Plasmon Resonance \\
\hline SMs & Small Molecules \\
\hline ss DNA & Single stranded DNA \\
\hline
\end{tabular}




\section{Contents}

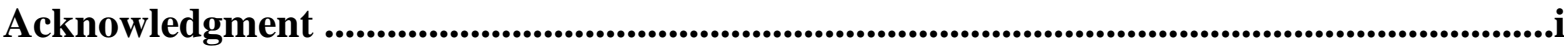

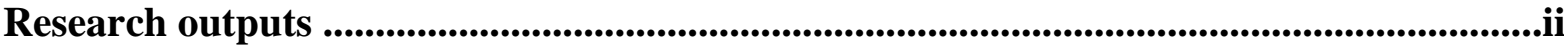

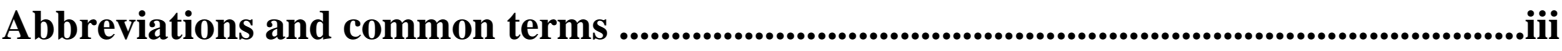

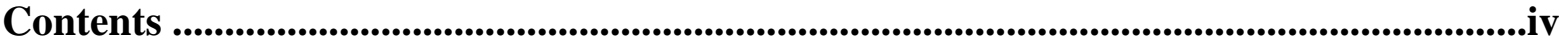

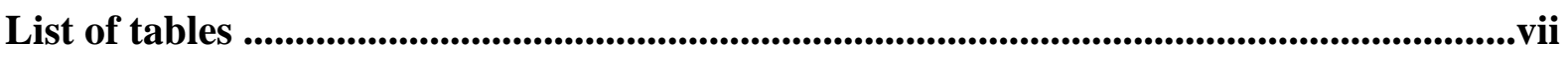

List of figures and schemes .......................................................................................................................viii

1. Introduction .................................................................... 1

1.1 Background............................................................. 3

1.1.1 Methamphetamine: The problem................................. 3

1.1.2 Oral fluid as a preferred matrix of detection of drugs of abuse........... 6

1.2 Biosensors.................................................................. 11

1.2.1 Optical biosensors............................................. 14

1.2.2 Colorimetric biosensors.............................................. 16

1.2.3 AuNP aggregation assays....................................... 16

1.2.3.1 Colloidal AuNPs stabilization: DLVO and non-DLVO forces........ 17

1.2.3.2 Strategies for AuNPs colloidal stabilization...................... 18

1.2.3.3 Mechanisms of AuNP aggregation.............................. 19

1.3 Aptamers................................................................ 24

1.3.1 Production of aptamers by SELEX.............................. 28

1.4 Aptasensors........................................................... 31

1.4.1 AuNPs based aptasensors for small molecule detection................ 33

1.4.2 Colorimetric aptasensors for drugs of abuse detection................ 37

1.5 Quantifying colorimetric aptasensors.................................. 40

1.6 Challenges of small molecule detection with AuNPs aptasensors............. 41

1.7 Objectives, research questions and knowledge gaps..................... 42

2. General methodology..................................................... 46

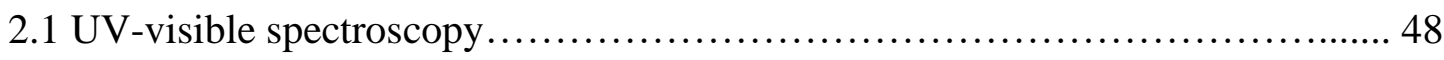

2.2 Circular dichroism................................................... 50

2.3 Dynamic light scattering and Zeta potential................................ 52

2.4 Isothermal titration calorimetry.......................................... 56 
3. Accounting for interaction kinetics between gold nanoparticles and aptamers enables high performance colorimetric sensors.............................59

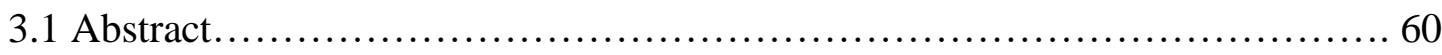

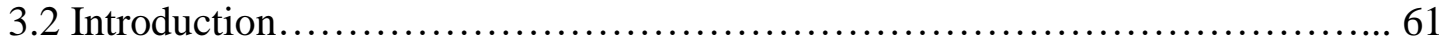

3.3 Experimental section................................................ 64

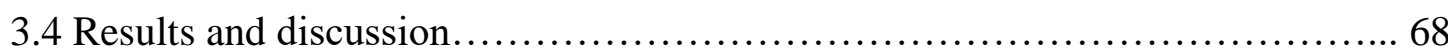

3.5 Chapter conclusion.................................................. 86

4. AuNP SELEX generated aptamers for the detection of methamphetamine using

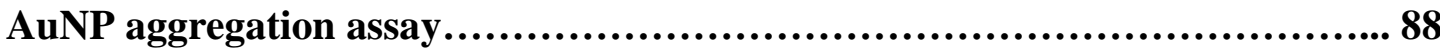

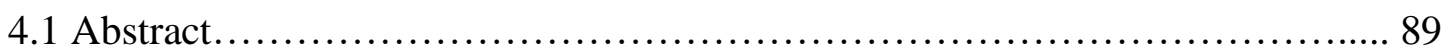

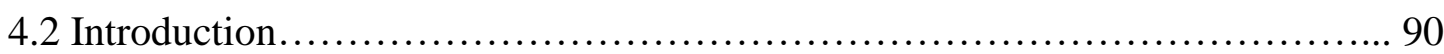

4.3 Experimental section................................................. 92

4.4 Results and discussion............................................... 92

4.5 Chapter conclusion................................................... 104

5. The nature of interferences when applying colorimetric aptasensors in human saliva.............................................................................. 106

5.1 Abstract.................................................................. 107

5.2 Introduction........................................................ 108

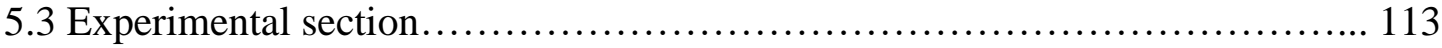

5.4 Results and discussion.............................................. 116

5.5 Chapter conclusion..................................................... 149

6. Fabrication and validation of a cost-effective and sensitive handheld light meter device for the detection of methamphetamine................................. 152

6.1 Abstract........................................................... 153

6.2 Introduction......................................................... 154

6.3 Results and discussion............................................... 155

6.4 Chapter conclusion.............................................. 165

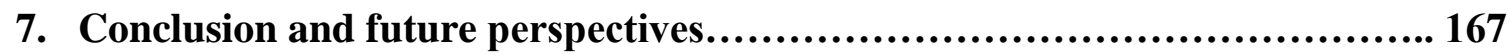

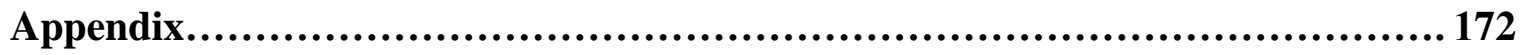




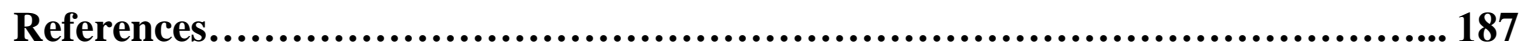




\section{List of tables}

Table 1.1 List of conventional techniques that are normally used to detect drugs/confirm drug tests

Table 1.2 A comparison of different parameters existing among most widely used OFD test kits........................................................................ 10

Table 3.1 Sequences of the used oligonucleotides (in 5' $-3^{\prime}$ 'direction) .................... 65

Table 3.2 Comparison between sensors for methamphetamine detection reported in the

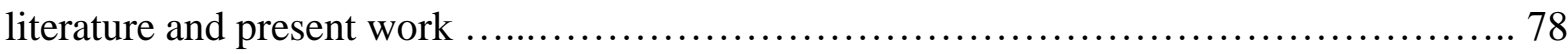

Table 3.3 Summary of main aptamer sequences used, assay formats used and LOD achieved ...... 80

Table 4.1 Summary of main aptamer sequences used, characteristics and LOD achieved ........... 102

Table 5.1 The measurement of meth detected in oral fluid ........................ 133

Table 5.2 The spiked vs predicted concentrations obtained when meth was spiked before dilution and pre-treatment to evaluate real world application suitability

Table 5.3 Spiked vs predicted values when referencing against passive drooling collection method of saliva 136

Table 5.4 Spiked vs predicted values when referencing against Quantisal collection method of saliva

Table 5.5 Details of the sample mixtures containing a mixture of 3 drugs

Table 5.6 Calibration of the obtained signal from the 42-mer aptamer in saliva samples that were spiked with meth after dilution/pre-treatment 146

Table 5.7 Calibration of the obtained signal from 42-mer aptamer after spiking meth prior to pre-treatment in saliva 148

Table A1 Zeta-potential values of 34-mer meth binding aptamer and 34-mer random aptamer with and without meth 183 


\section{List of figures and schemes}

Figure 1.1 Chemical structure of methamphetamine and its metabolite amphetamine...... 5

Figure 1.2 Schematic showing the basic components of a biosensor .................. 12

Figure 1.3 Classification of biosensors and /or aptasensors ......................... 13

Figure 1.4 Illustration of the basic difference between SPR and LSPR modes ........... 15

Figure 1.5 Physical and chemical properties of AuNPs and schematic illustration of the

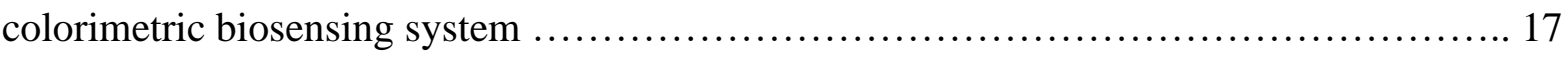

Figure 1.6 Schematic illustration of AuNPs colloidal stabilization A) electrostatic B) steric C) combination of electrostatic and steric forces

Figure 1.7 Schematic showing the basic principle of the interparticle cross-linking induced aggregation mechanism

Figure 1.8 Schematic of interparticle cross-linking mechanism without an external crosslinker. 21

Figure 1.9 Schematic of non-cross-linking mechanisms

Figure 1.10 Schematic showing the unique conformational change undergone by aptamers induced by the target. 25

Figure 1.11 The schematic illustration of the basic structure of a DNA

Figure 1.12 Schematic illustration of the traditional SELEX procedure that uses bead immobilized target for aptamer selection

Figure 1.13 Schematic of an AuNPs based aptasensors for colorimetric detection of analytes.

Figure 1.14 Mechanism of AuNPs aptasensors for ATP detection 34

Figure 1.15 Multiplexed colorimetric detection of cocaine and meth by a novel aptasensors

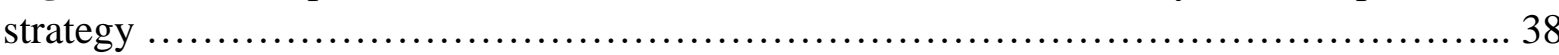

Figure 1.16 Colorimetric DNAzyme based aptasensors for the detection of meth .......... 40

Figure 2.1 Schematic showing the main components of a UV-Vis spectrometer .......... 49

Figure 2.2 Schematic showing the working of a CD spectrophotometer $\ldots \ldots \ldots \ldots \ldots \ldots \ldots . \ldots$

Figure 2.3 Schematic showing the working principle of a DLS instrument ............... 53

Figure 2.4 Schematic representation of the theory of zeta potential $\ldots \ldots \ldots \ldots \ldots \ldots \ldots \ldots . \ldots 5$

Figure 2.5 Schematic showing the principle of an ITC instrument ..................... 57

Scheme 3.1 Possible mechanism of the signal enhancement for the aptamer dissociation assay (target last) format compared to the aptamer association assay (target first) format ... 64 
Figure 3.1 Colorimetric response of the parent 75-mer and truncated 34-mer towards a range of meth concentrations using the aptamer dissociation assay format $\ldots \ldots \ldots \ldots \ldots \ldots \ldots \ldots . \ldots 1$

Figure 3.2 Secondary structure of the 75-mer aptamer predicted by the Mfold program .... 72

Figure 3.3 (A) Salt titration curve showing the optimal salt concentration shifting towards right as a function of time for the 34-mer meth binding aptamer (B) Salt titration curve with the target showing the decreased sensitivity towards met hover time $\ldots \ldots \ldots \ldots \ldots \ldots \ldots \ldots . \ldots \ldots$

Figure 3.4 Plot of zeta potential vs time showing the increasing stability of aptamer adsorbed AuNPs and decreasing sensitivity in the presence of meth over time

Figure 3.5 A) Colorimetric aptasensors response comparison for the 34-mer meth binding aptamer using the aptamer association assay vs dissociation assay B) 34-mer aptamer association assay response along with bare and random 34-mer controls and inset showing the linear fit.

Figure 3.6 Specificity examinations of interfering molecules methamphetamine (MA), amphetamine (AMP), ephedrine (EPH), pseudoephedrine (PSEUDO), methylenedioxyamphetamine (MDA), 3,4-methylenedioxymethamphetamine (MDMA) ...79

Figure 3.7 CD experimental data for the 34-mer meth aptamer alone and in the presence of meth............................................................................ 81

Figure 3.8 A) Raw ITC thermogram B) Integrated heat profile of 34-mer ................ 83

Scheme 4.1 Illustration of the AuNP SELEX procedure which uses in-solution selection of aptamers for small molecule targets that are non-immobilized and free in solution

Figure 4.1 OM-C6 responds best among the two other new generation SELEX developed

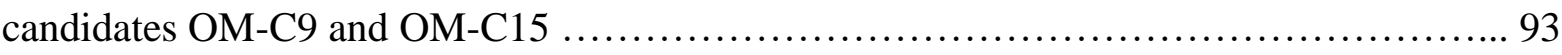

Figure 4.2 Salt optimization graph for the 81 -mer ................................ 94

Figure 4.3 Colorimetric sensing response of 75-mer and 81-mer aptamers with bare AuNPs and negative control aptamer ICPD-04 f...................................... 96

Figure 4.4 M-fold predicted structures of parent 81-mer and its two halves constituting the intact double stranded probable binding regions, 42-mer and 39-mer

Figure 4.5 Colorimetric response of the parent 81-mer versus the two constituent halves, 42-

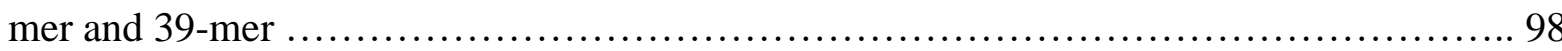

Figure 4.6 Salt optimization curve of the 42 -mer ................................. 99

Figure 4.7 Colorimetric aptasensors response of the 42-mer towards meth using the association and dissociation assay to show the effect of change pf assay sequence

Figure 4.8 Summary of colorimetric responses of the affinity-based SELEX selected aptamers 75-mer and truncated 34-mer and the 81-mer and the truncated 42-mer........

Figure 4.9 CD experimental data for 42-mer meth aptamer alone and in the presence of meth.

Figure 5.1 The three major pairs of salivary glands 
Figure 5.2 Illustration of major modes of transportation of biomolecules and small molecules from blood to saliva

Figure 5.3 The image shows the composition, function and the factors affecting salivary flow and composition

Figure 5.4 The different methods of saliva pre-treatment and processing to remove interfering proteins that were tried and tested

Figure 5.5 Graphical and visual illustration of the problem with application of undiluted/untreated whole saliva into the AuNP aggregation assay

Figure 5.6 (A) Image of pre-treated saliva sample diluted with buffer after sonication for 30 minutes (B) Image showing that AuNPs could only be susceptible to aggregation to salt $(0.5$ $\mathrm{M} \mathrm{NaCl}$ ) when whole saliva was diluted more than 150 times

Figure 5.7 Kinetics of aggregation in real undiluted filtered (reduced mucins) saliva

Figure 5.8 Kinetics of electrolyte interference vs protein interference

Scheme 5.1 Illustration of proposed mechanisms of interference from the salivary matrix and AuNPs aggregation aptasensors

Figure 5.9 Graphical illustration of the increasing sensitivity towards increasing ionic strength with each targeted protein removal strategy

Figure 5.10 UV-Vis characterization of interactions between AuNPs and salivary matrix under different targeted processing strategies employed to pre-treat saliva

Figure 5.11 DLS characterization of the effect of two targeted protein removal approach on AuNPs aptamer samples

Figure 5.12 Summary of DLS size investigations of bare AuNPs with and without aptamer in diluted untreated saliva and in the final HCS obtained after 2x syringe filtration .130

Figure 5.13 Plot clearly showing the improvement in response obtained by using the POC technique to remove proteins 131

Figure 5.14 Salt optimization of 34-mer aptamer in oral fluid

Figure 5.15 Colorimetric response of the 34-mer

Figure 5.16 Graphical illustration of the effect of filtration on the concentration of meth ..134

Figure 5.17 Graphical representation of spiked vs predicted values correlation in undiluted saliva

Figure 5.18 Illustration of the signal suppression in saliva medium collected via two different methods, passive drooling and Quantisal methods 139

Figure 5.19 Specificity examinations of unknown mixtures of interfering molecules in saliva with the 34-mer dissociation AuNPs assay 140

Figure 5.20 Specificity examination in saliva samples containing singe interfering compound. 
Figure 5.21 Optimum salt concentration/ionic strength needed for the optimum response of the colorimetric sensor was found to be $48 \mathrm{mM}$

Figure 5.22 Optimum salt concentration/ionic strength needed for the optimum response of the colorimetric sensor

Figure 5.23 Colorimetric response of 42-mer aptamer in saliva that was spiked with meth after dilution and pre-treatment

Figure 5.24 A) Visual B) Graphical comparison of sensing responses of traditional vs AuNP SELEX selected aptamers in saliva

Figure 5.25 Colorimetric response obtained from old vs new SELEX selected aptamers at meth concentrations spiked prior to pre-treatment of saliva samples ....

Figure 6.1 A) Concept of the light proof attachment containing the light source and sample holder, parts labelled B) the mini Bluetooth digital light meter detector C) the final device that was made by attaching the light proof attachment with the digital light meter detector D) Close-up image of the LED attached to the lid and closing over the sample vial

Figure 6.2 Graphical illustration of stability study of the light source and detector over time to confirm the stability of the set up

Figure 6.3 A) Colorimetric response of the 42-mer meth aptasensors in terms of absorbance at $520 \mathrm{~nm}$ obtained from the standard plate reader instrument B) Colorimetric response obtained from the light meter device

Figure 6.4 Calibration curve of the 42-mer aptasensors obtained with A) the standard plate reader absorbance $\mathrm{B}$ ) the light meter device 164

Figure A1 Colorimetric screening of a family of meth aptamers obtained from Auramer Bio.

Figure A2 (A) UV-Vis characterization of AuNPs in dispersed and aggregated states (B) DLS size characterization for AuNPs and (AuNPS, 75-mer aptamer) in aqueous media ...173

Figure A3 (A) Salt dependent aggregation for AuNPs mixed with increasing 75-mer aptamer coverage (B) Sensitivity optimization of various concentrations of aptamer (33, 66 and 99 $\mathrm{nM}$ ) such that the stoichiometric ratio of [Aptamer] : [AuNPs] are 3:1, 6:1 and 9:1 ......174

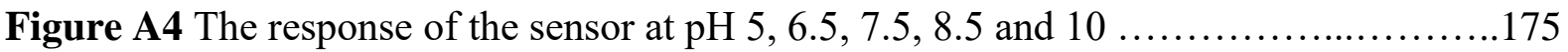

Figure A5 Salt optimization graph for A) 75-mer dissociation assay B) 34-mer dissociation assay C) 34-mer association assay D) 34-mer aptamer in oral fluid.... 176

Figure A6 Linear fit curves of A) 75-mer and B) 34-mer

Figure A7 Control studies to evaluate the effect of meth on the adsorption of aptamers ....179

Figure A8 Bare AuNPs control for the different interfering molecules at $200 \mathrm{nM}$

Figure A9 SG assay with a methamphetamine titration against 75-mer aptamer. 
Figure A10 SG assay with a methamphetamine titration against 34-mer aptamer..........182

Figure A11 Predicted M-fold 2-D structure of 34-mer random sequence ....................... 184

Figure A12 (A) Illustration of colour changes occurring across samples over a range of meth concentrations from $0-1500 \mathrm{nM}$ (B) Aggregation plot showing ratio of absorbances at $670 / 521$ vs concentration of meth (nM) to compare the sensing responses between parent and truncated sequences using the dissociation and association assay 185

Figure A13 Comparison of colorimetric responses from the aptamer association and aptamer dissociation assay formats in oral fluid 186 
CHAPTER 1

\section{Introduction}




\section{Chapter overview}

This chapter will introduce the problem of methamphetamine in New Zealand, then move on to describing the current state of the art detection methods and matrices along with their shortcomings. The chapter will then introduce the concept of biosensing and its various transduction modes particularly focusing on colorimetric biosensors and discussing about the conventionally used bioreceptors while introducing the concept of aptamers. The chapter will briefly discuss the various kinds of analytes detected by biosensors especially focusing on small molecule targets and the challenges associated with their detection. This chapter shall discuss in detail the aptasensors developed for small molecules especially focusing on drugs of abuse such as cocaine and methamphetamine molecule. The need for a point-of-care detector for colorimetric aptasensors for quantitative detection is also discussed in the end. Finally, this chapter shall state the goals and identify the research questions or challenges of this project. 


\subsection{Background}

\subsubsection{Methamphetamine: The problem}

Methamphetamine (meth) was first synthesized in Japan, in 1893 by a Japanese chemist, from another stimulant known as ephedrine. However, meth was difficult to synthesize until 1919 when another Japanese chemist Akira Ogata streamlined the process. ${ }^{1,2}$ Ephedrine was reduced using red phosphorus and iodine to make crystals of meth giving the world its first crystal meth. Early on, meth was used as a treatment for narcolepsy, asthma and as a weight loss drug. The crystal meth could easily be dissolved in water which made administration very easy by injection. It was widely used during the World War II to keep troops awake and fighting by both sides for example the Japanese administered meth to Kamikaze pilots before their suicide missions in the war. In the Vietnam war methamphetamine used by American troops alone exceeded the amount used by the rest of the world combined. The problem became serious when meth was being used widely after the war not just by the military, but it crept into the lives of common public which eventually led to meth being outlawed in the USA in $1970{ }^{2,3}$ Until the late 1980's illicit meth abuse was endemic to California and later it's illegal use spread to all other parts of the country. It's entry into the New Zealand drug market has started in the late 1990's. Before that, NZ like its counterparts Britain and USA began aggressively marketing the derivative of meth which is amphetamine as an active ingredient of oral medication for weight loss etc. It is since then that the unchecked rise and abuse of first amphetamine and then meth began leading to the illegal synthesis and manufacture later in the 1990's and the first clandestine methamphetamine laboratory was discovered in 1996 by the police in NZ. ${ }^{4}$ After the arrival of meth which is referred to as $\mathrm{P}$ or 'pure' locally in NZ, the drug culture of NZ changed. A shift from the demand for opiates, cannabinoids and cocaine was observed owing to the supply problems which depends on the cross-border trafficking. Whereas, the supply of meth could be uninterrupted because of the possibility of manufacturing easily via locally available precursors. Moreover, meth differed from other stimulant drugs in its ease of administration such as the possibility of smoking crystal meth proved more convenient compared to intranasal or oral administration which was necessary in case of other drugs. ${ }^{5,6}$

The meth problem is indicative of a far dangerous situation than the simple drug abuse related selfdestruction of an individual that is mostly limited to the abuser. Meth abuse causes an individual 
to be violent (most of the time). The increase in crime rates induced by meth is a matter of worry for the whole community. Research shows that more than two thirds of people who are in prison have admitted to using drugs while in prison and the remaining one-third admitted being under the influence of drugs during committing crimes. ${ }^{7}$ In addition, studies have also shown that most fatal automobile accidents have happened because of the involvement of drivers who have been under the influence of drugs. Therefore, there is an urgent need for drug testing at roadsides and workplaces to ensure a meth free safe environment. Workplace routine drug testing should be carried out to ensure and promote safe workplace culture, health, and productivity amongst employees. Moreover, drug testing has been a part of every stage of criminal justice system and is also a mandatory and integral part in sports.

\section{Chemical and physical properties of meth}

The IUPAC id of meth is N-Methyl-1-phenylpropan-2-amine and it has a molecular weight of 149.237 g/mol. Dextromethamphetamine (D-meth) and levomethamphetamine (L-meth) are the two isomers of methamphetamine. D-meth is the more potent and powerful central nervous system stimulant. Rare conditions such as attention deficit disorder and short-term treatment of obesity utilizes d-methamphetamine as treatment, and it is pharmaceutically derived. Synthetic techniques of synthesis for the illicit manufacture of methamphetamine lead to the production of relatively pure D-meth which can be easily manufactured in clandestine laboratories. Some techniques may also produce D/L-mixtures. These illicitly derived methamphetamine is available for a relatively low cost owing to their ease of production. L-meth on the other hand is relatively less potent and is sold over the counter as a nasal decongestant product. ${ }^{8}$

Meth is mainly used as a recreational drug and is a potent central nervous system stimulant. It is structurally related to its metabolite amphetamine differing only by the presence of a methyl group (Figure 1.1). Both meth and amphetamine are used to treat attention deficit hyperactivity disorder (ADHD) and narcolepsy. In the USA oral meth is sold by the trade name Desoxyn over the counter for the treatment of ADHD as well as obesity. Whereas in Canada amphetamine is the active component of several medications such as Adderall and Dexedrine prescribed for the treatment of ADHD. The mechanism of action of both meth and amphetamine is same, that is both increases the levels of monoamine neurotransmitters which are dopamine, serotonin and norepinephrine in the brain causing that stimulation of the central nervous system. ${ }^{9}$ Both meth and amphetamine are 
known as psychostimulants because of their ability to increase dopamine concentrations in brain which is the control center for body movements, motivation and pleasure. However, meth is a more potent psychostimulant compared to amphetamine. The effects of D-meth may last for 6-12 hours. Generally, meth users remain hyperactive, restless, and irritable after the initial 'rush', some may become more aggressive and violent which have unpleasant consequences. Long term effects also include oral and dental disintegration, severe itching, and paranoia. Short term health effects of meth include insomnia, loss of appetite and weight loss, elevated body temperature and heart rate. Withdrawal symptoms include depression, anxiety, psychosis, and serious drug cravings. Unfortunately, there are no approved medications that can be used to treat meth addiction. The only respite lay in behavioral and motivational therapies. ${ }^{10}$ It is considered a scheduled drug due to its high potential for addiction and abuse in most countries.
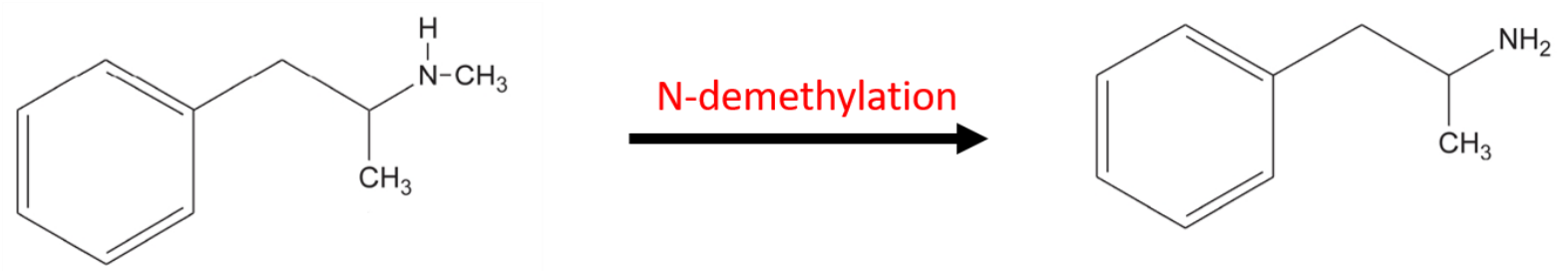

Figure 1.1 Chemical structure of methamphetamine and it's metabolite amphetamine

Meth can be rapidly absorbed by all administration routes such as smoking, intravenous, intranasal an oral administration. However, the onset of effects is fastest via intravenous and smoked routes. Meth is primarily metabolized in the liver by three chemical processes: aromatic hydroxylation, $\mathrm{N}$-demethylation to form amphetamine and deamination. The pKa of meth is 10.1 which is due to the basic nitrogen moiety in meth's chemical structure. The high lipophilicity of meth and its low plasma-protein binding (10-20\%) influences its distribution and excretion processes. Acidic urine favours meth concentration and reduces its half-life in the body whereas basic urine slows down excretion and prolongs residence time in the body Due to meth's small size, lipophilicity and low plasma protein binding, it concentrates into saliva (slightly acidic matrix) due to ion trapping. Also, since saliva is more acidic when compared to plasma, the concentration of meth is enhanced in saliva compared to in plasma. 


\subsubsection{Oral fluid as a preferred matrix of detection of drugs of abuse}

Conventionally, the preferred matrix for drug testing has been urine and blood/plasma. However, alternative drug testing matrices such as oral fluid, hair and sweat are being investigated owing to their advantages over the conventionally used matrices. For example, the alternative matrices offer non-invasive sampling for testing. Urine may also be considered to be a non-invasive sample but it does involve invading privacy of the donor if the collection needs to be monitored to rule out opportunities of adulteration/cheating. The alternate matrices may have some disadvantages for example hair, which may provide the drug use history of a donor but it may also mislead if the donor's hair has only been in contact by hand of a chronic meth user or the donor has been in the vicinity of drug vapours inhaled by others because the hair could be externally contaminated in that way and lead to unnecessary woes for the donor. Similarly sweat as a matrix may be collected by sweat patches but limited research/knowledge exists about the interpretation of drug concentration correlations in the patches. ${ }^{11,12,13}$

In this context oral fluid is rapidly emerging as a promising alternative for detecting drugs of abuse owing to the following advantages over other matrices:

1) Complete non-invasive sample collection even in terms of privacy and can be carried out by any person or the donor himself with very little training required

2) Guarantees relative safety in sample handling as opposed to blood

3) Saliva offers monitoring of instant/ most recent drug use due to oral contamination if the drug is administered orally (eg. smoked) whereas the appearance of drug in blood is only after a few hours of oral administration. This facilitates detection of drugs such as amphetamines, marijuana, cocaine etc.) at high concentrations in saliva

4) Saliva offers detection of drugs in their unmetabolized parent form because drugs in saliva appear after passive diffusion through the blood membrane which facilitates easy extraction and confirmation without the need for derivatization 
There are also some disadvantages of saliva as a matrix of detection:

1) The immunoassay kits for saliva drug tests are designed for testing the drug metabolites and hence the unmetabolized form of the drug found in saliva may be a problem.

2) The elevated concentrations of drug in oral fluid after smoking/ oral administration is not indicative of the level of impairment as it would not indicate the actual plasma levels. Therefore relating a dose concentration with that of saliva concentration is a problem due to oral contamination

3) Shorter drug detection windows compared to urine

4) Need for greater assay sensitivity

However, the advantages outweigh the disadvantages listed and recently oral fluid seems like an attractive and cost-effective alternative to traditionally used matrices especially for screening large populations.

\section{Saliva/plasma correlation and kinetics}

Saliva/plasma concentrations can be theoretically calculated by the mathematical model developed using the following equation $1.1^{11}$

$$
\text { Basic Drugs }-\frac{S}{P}=\frac{1+10^{(p K a-p H s)} x f p}{1+10^{(p K a-p H p)} x f s}
$$

Where, $\mathrm{S}=$ concentration of drug in saliva, $\mathrm{P}=$ concentration of drug in plasma, $\mathrm{pKa}=\mathrm{pKa}$ of drug, $\mathrm{pHs}=\mathrm{pH}$ of saliva, $\mathrm{pHp}=\mathrm{pH}$ of plasma, $\mathrm{fp}=$ free unbound fraction of drug in plasma, $\mathrm{fs}=$ free unbound fraction of drug in saliva

Most pharmacokinetics studies have been done based on plasma drug concentrations and if the equations could be shown to be predictable then the existing databases on plasma drug concentration information could be used for predicting saliva drug concentrations and then it could be established as a strong alternative matrix for detecting drugs of abuse. ${ }^{11,14}$ 
Limited reports exist on methamphetamine detection in saliva. In the body, methamphetamine is metabolized into amphetamine via $\mathrm{N}$-demethylation. Reports on amphetamine detection in saliva and plasma was conducted by Wan et al. after administering $10 \mathrm{mg}$ amphetamine orally to patients. After determining the drug concentrations in saliva and plasma the S/P ratio was calculated. ${ }^{15}$ The calculated ratio was always greater than that theoretically predicted because of the oral contamination factor involved during oral administration. However, it was claimed that the study suggested that the mathematical model could be used to predict amphetamine concentrations in plasma when the saliva concentrations was known. Eventually it was reported by Vapaatalo et.al ${ }^{11}$ that low concentrations of amphetamine found in saliva made it difficult to do Thin layer chromatography (TLC) to determine amphetamine concentrations in saliva suggesting saliva as an unsuitable matrix for detecting amphetamine.

However, methamphetamine has been detected in saliva by Kajutani et al. and Suzuki et al. ${ }^{11}$ Suzuki et al. reported detecting methamphetamine in saliva of meth users after two days of last use. The concentrations recovered in saliva after extraction procedures was detectable in 3 out of the 19 saliva samples tested. Amphetamine could not be detected at all in these saliva samples.

It has been reported that the collection procedure affects the saliva concentrations of drugs. Many collection procedures exist which can be divided into two main categories: stimulated and unstimulated. While unstimulated saliva collection involves no contamination by any external factors and the $\mathrm{pH}$ can be pretty much controlled, stimulated saliva collection on the other hand may provide enough sample volume and quick collection times, these samples are often contaminated with external factors such as by the components of the stimulant use which is mainly citric acid based. This also results in alteration of the $\mathrm{pH}$ and the deposition of drug may get affected. $\mathrm{pH}$ alteration may also affect the subsequent detection especially when the detection method used is $\mathrm{pH}$ sensitive. However, studies on effect of saliva collection methods are very limited. ${ }^{16}$

\section{Conventional techniques for the detection of meth in oral fluid}

Existing techniques to detect methamphetamine include Gas Chromatography-Mass Spectroscopy $(\mathrm{GC}-\mathrm{MS})^{8}$, Liquid Chromatography-MS (LC-MS $)^{17}$, Capillary Electrophoresis $(\mathrm{CE})^{18}$, Immunoassays ${ }^{19}$ etc. ${ }^{20,21,22,23}$ Techniques such as mass spectrometry combined with 
chromatography have been reported to be more reliable than immunoassays. A study involving enzyme-linked immunosorbent assay (ELISA) for the detection of three main drugs namely meth, amphetamine and methylenedioxymethamphetamine (MDMA) in oral fluid samples was conducted and a concentration of $>10,000 \mathrm{ng} / \mathrm{mL}$ was needed to produce a positive response which clearly indicated the unsuitability of these assays for application in real cases as these concentrations are in huge excess of what is normally found in forensic samples. ${ }^{24}$ Whereas GCMS have been used to detect 30 psychoactive drugs including meth and metabolites in oral fluid samples with a Limit of detection (LOD) of $6.9 \mathrm{ng} / \mathrm{mL} .{ }^{25}$ Liquid chromatography-tandem mass spectrometry (LC-TMS) has been performed to screen 32 drugs in oral fluid with LOQs as low as $3 \mathrm{ng} / \mathrm{L} .{ }^{26}$ Similarly LC-MS has been used for the detection of drugs in oral fluid with a cut-off value of $3 \mathrm{ng} / \mathrm{mL} .{ }^{27}$ A promising analytical techniques that can be used for detection of drugs of abuse could be Selective ion flow tube mass spectrometry (SIFT-MS). ${ }^{28}$ However, no such report of its application for detection of methamphetamine has been found in the literature. These techniques are nevertheless sensitive and highly reliable but not suitable for POC testing and are definitely limited by cost and need for trained expertise. Table 1.1 lists some of the conventionally used techniques for drugs of abuse detection and their sensitivities.

Table 1.1 List of conventional techniques that are normally used to detect drugs/confirm drug tests

\begin{tabular}{|l|l|l|}
\hline Method & Analyte & LOD $(\mathbf{n g} / \mathbf{m L})$ \\
\hline Immunoassay & Meth, Amphetamine, MDMA & 10,000 \\
\hline GC-MS & Meth and its metabolites & 6.9 \\
\hline LC-TMS & Meth and other psychoactive drugs & 3 \\
\hline LC-MS & Meth & 3 \\
\hline
\end{tabular}

Commercial meth oral drug test kits that are available on the international market are listed in Table 1.2. The data in Table 1.2 has been obtained from a study conducted by M. Asbridge and R. Ogilvie on roadside oral fluid drug testing in Canada. ${ }^{29}$ The different devices have been compared on the basis of cut off values which is the drug concentration level at which the device can detect a given drug, sensitivity and specificity. It seems a lot of variations exist among the 
different drug test kits which makes it inaccurate and unadvisable to use these kits independently without supporting lab based tests. ${ }^{30}$

Table 1.2 A comparison of different parameters existing among most widely used OFD test kits.

\begin{tabular}{|c|c|c|c|c|c|}
\hline Device & Mechanism & $\begin{array}{l}\text { Cut-off } \\
\text { (ng/mL) }\end{array}$ & $\begin{array}{l}\text { Sensitivity } \\
(\%)\end{array}$ & $\begin{array}{l}\text { Specificity } \\
(\%)\end{array}$ & $\begin{array}{l}\text { Test time } \\
\text { (mins) }\end{array}$ \\
\hline $\begin{array}{l}\text { Dräger Drug } \\
\text { Test 5000® }\end{array}$ & $\begin{array}{l}\text { OF is collected on a swab on a } \\
\text { test cassette, which is inserted } \\
\text { into a reader. }\end{array}$ & 35 & $50-86.4 \%$ & $94-100 \%$ & 9 \\
\hline Alere DDS2® & $\begin{array}{l}\text { A test cartridge is inserted in an } \\
\text { analyzer and OF sample is } \\
\text { inserted in the test cartridge. }\end{array}$ & 50 & - & - & 6 \\
\hline DrugWipe 5S® & $\begin{array}{l}\text { A collection swab is saturated } \\
\text { and paired with a test body. A } \\
\text { liquid ampule with buffer } \\
\text { solution is added to the test } \\
\text { body. The results can be read } \\
\text { from test device or the } \\
\text { electronic DrugRead reader. }\end{array}$ & 25 & $60 \%$ & $96.5-100 \%$ & $3-8$ \\
\hline RapidSTAT@ & $\begin{array}{l}\text { A collection swab with aroma } \\
\text { field is saturated, placed in a } \\
\text { buffer solution, agitated, and } \\
\text { removed. Seven drops of the } \\
\text { buffer fluid is added to each } \\
\text { well of the test device and left } \\
\text { for } 4 \text { minutes. The result can be } \\
\text { read from the test device or the } \\
\text { electronic reader. }\end{array}$ & 25 & $76.2-100 \%$ & $71-99 \%$ & $7-12$ \\
\hline
\end{tabular}


In summary, traditional, and existing methods for detecting meth in plasma, urine, saliva etc. employ expensive apparatuses and tedious procedures. They are inconvenient because they involve bulky, expensive, and complicated instruments. Their operation requires trained experts. These reasons make them unsuitable for applications in POC devices ${ }^{31}$ for road side testing or work place testing. Moreover, the commercial drug test kits which overcome the limitations of the traditional procedures are wrought with problems such as reliability and sensitivity. However, the increasing meth abuse by people and the resulting activities after, such as driving and working under its influence is triggering the need for rapid, simple and cost-effective assays for meth detection. ${ }^{31}$ In this context, biosensors will be briefly described, a popular and promising platform for detection of small molecules in biological matrices with immense scope for miniaturization.

\subsection{Biosensors}

Biosensors are bio analytical devices which can convert a biological or chemical event occurring at their surface or in their vicinity into an interpretable electrical signal. Unique attributes of a biosensor include high specificity, sensitivity, reproducibility, robustness, or independence from

physical parameters like $\mathrm{pH}$, temperature etc., simplicity and efficiency. ${ }^{32}$ Biosensors are desirable owing to their rapid and accurate detection capabilities of environmental pollutants, pathogens, hormones drugs etc.

A biosensor consists of four basic components as shown in Figure 1.2, an analyte, bio-receptor or probe or bio-recognition element (BRE), a signal transduction element (STE) or transducer and a signal output or display. Several BRE's or probes can be used to fabricate biosensors such as, antibodies, DNA aptamers, enzymes, microbes, polymers etc. The BRE and STE are in close proximity to each other such that the STE is able to convert or transduce any interaction occurring between the affinity pairs i.e. the BRE and the analyte, into a useful electrical signal that can be displayed and analyzed by the output. ${ }^{33}$ 


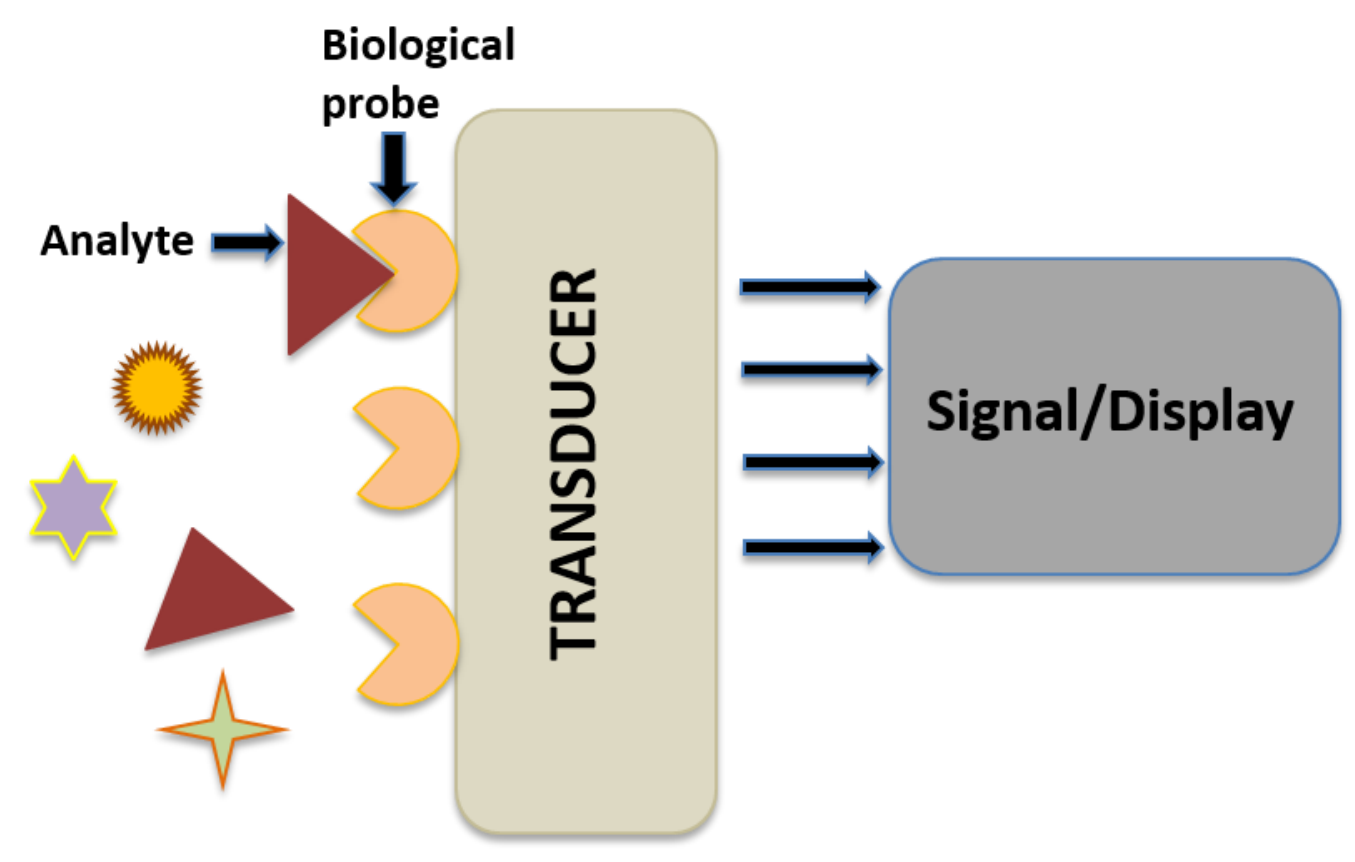

Figure 1.2 Schematic showing the basic components of a biosensor.

\section{Classification of biosensors}

Biosensors can be classified on the basis of their two main components: a) BRE and/or b) on the basis of STE (Figure 1.3) ${ }^{34}$ Based on the choice of the BRE's biosensors can be classified into molecular imprinted polymer-based biosensors, aptasensors and immunosensors. ${ }^{35}$ On the basis of transducer used, biosensors can be grouped as electrochemical biosensors that convert a chemical or biological reaction into an electrical signals such as current, voltage, impedance etc., optical biosensors produce signals in the form of a visual colour change or change in refractive index (and plasmon peak) which is directly proportional to the concentration of analyte, mechanical/micromechanical biosensors records changes in oscillations (e.g. piezoelectric crystal), or pressure etc arising from mass bound on the sensor surface and convert them into a useful electrical signal. ${ }^{36}$ 


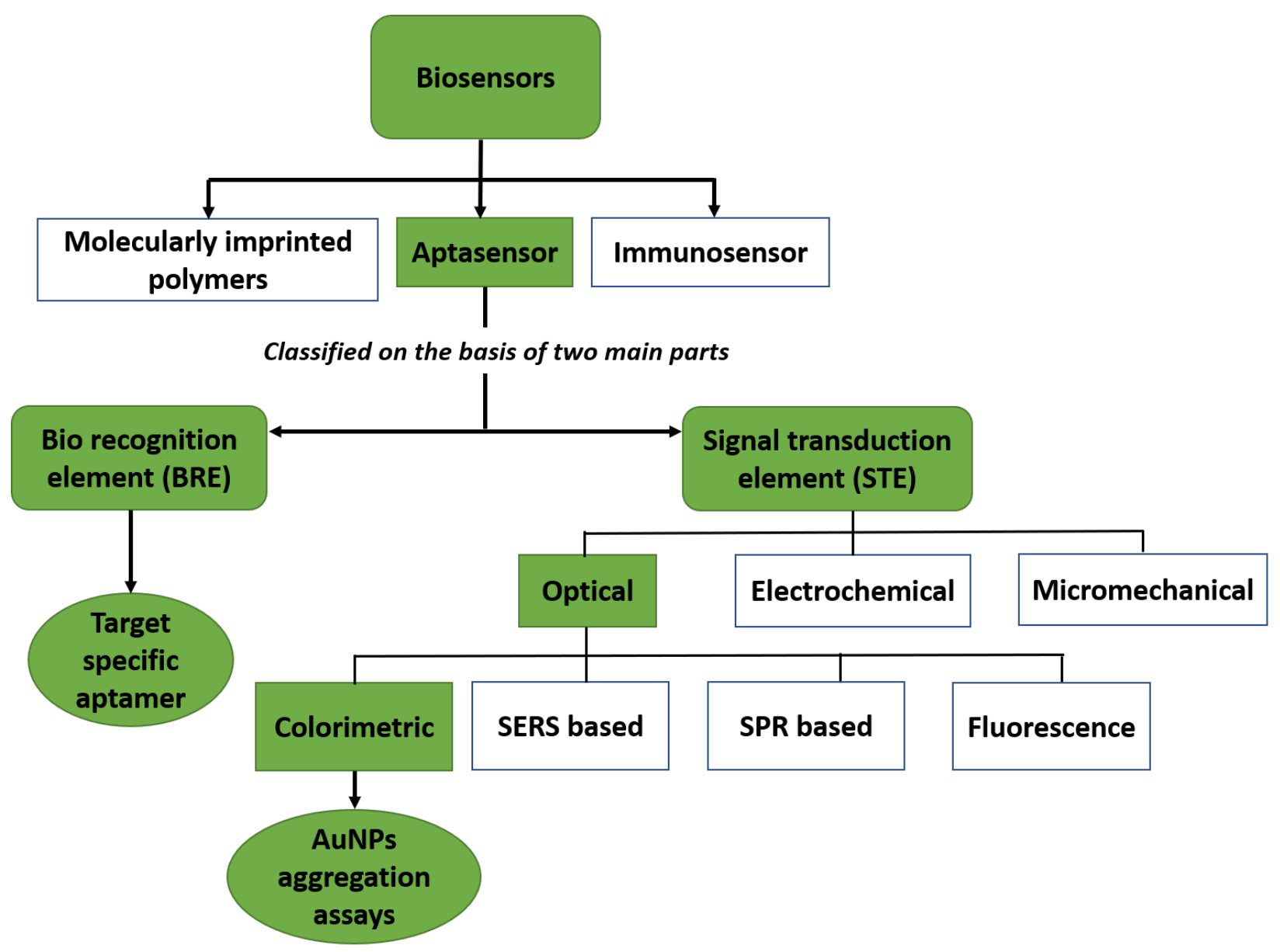

Figure 1.3 Classification of biosensors and/or aptasensors

\section{Brief History}

It is well known among biosensor enthusiasts and researchers that the first true biosensor was advanced by Prof. Leland C. Clark, Jr also referred to as the father of biosensors in 1956 for detection of oxygen and in 1962 he developed an enzyme based amperiometric biosensor for detection of glucose. These developments were followed by more discoveries which led to the first potentiometric biosensor for urea detection by Guilbault and Montalvo, Jr in 1969. Later on in 1975, the first commercial biosensor was developed by Yellow Springs Instrument and from then on till 1992 some major developments led to the first hand-held biosensor for blood glucose monitoring, iSTAT which was developed in 1992 and after that the rest is history. Exceptional progress has been made in the field of biosensors ever since and today it's the most widely applied field attracting scientists and researchers with an interdisciplinary background. ${ }^{32}$ 
Biosensors have a wide range of applications focused around improving the quality of life. They have applications in environmental monitoring, food safety, disease detection, drug detection etc. Due to the wide range of applications and the immense scope for scalability in biosensors, there has been a long-standing demand for portable and on-site diagnostic systems. Of the various transduction modes, optical biosensors have been most suitable for point-of-care diagnostics due to its ease of adaptability. ${ }^{37,38}$

\subsubsection{Optical biosensors}

Optical biosensors can be classified into colorimetric, surface plasmon resonance (SPR) based biosensors, Surface Enhanced Raman spectroscopy (SERS) based biosensors, and fluorescencebased biosensors. Of these, the colorimetric biosensors which are based on the localized surface plasmon resonance (LSPR) effect and the SPR based biosensors are the most popular. To understand LSPR it is important to briefly discuss the SPR effect as well. ${ }^{39}$

Surface plasmons (SP) occur when photons of an incident light wave interact with the valence electrons of metals leading to a collective oscillation of conduction band electrons that are in resonance with the incident light electric field. The plasmons propagate parallel to the metal surface, thereby forming an SP wave. The SP wave propagates on the boundary between the metal and the dielectric, exponentially attenuating in the dielectric. A change in the refractive index due to molecular interactions on the metal surface is detected by an SP wave in SPR based biosensors. When these plasmons are confined in a metal nanoparticle with dimensions comparable to those of the incident light and the particles free electrons are engaged in collective oscillation, the generated effect is called LSPR. The LSP which is a non-propagating SP is distributed in a small region around the particle and the LSPR can be adjusted by the particle properties i.e. shape, size and composition.

An SPR based biosensor operates on the principle of interaction of light with metal or conducting material present at the interface of two dielectric media (usually glass and liquid) at a specific angle which generates plasmons and a resultant dip in intensity of reflected light at a certain angle. This allows recording the change in the resonance angle which is proportionate to the analyte concentration. With this system it is possible to monitor biological interactions in real time without labelling, which is offered by only a few other techniques such as ellipsometry and quartz crystal 
microbalance. ${ }^{40}{ }^{41} \mathrm{~A}$ variety of analytes may be detected using SPR for example, detection of soluble vascular endothelial growth factor receptor ${ }^{42}$, mycotoxins ${ }^{43}$, antibiotics in milk ${ }^{44}$ as well as heavy metals such as Arsenic etc. ${ }^{45}$

In contrast to SPR based biosensors LSPR biosensors are based on the interaction of light with metallic nanostructures exploiting their unique optical properties which are absent in bulk metal structures. The major difference as illustrated in Figure 1.4, between the two is that excited plasmons oscillate locally on the nanostructures rather than along the metal/dielectric interface. ${ }^{38}$

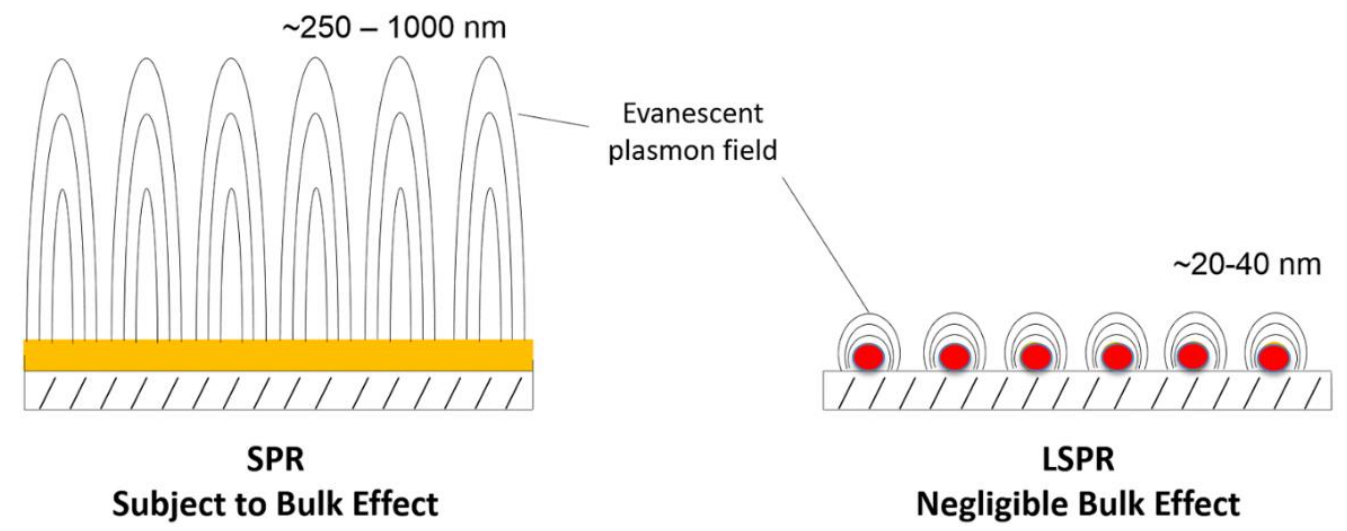

Figure 1.4 Illustration of the basic difference between SPR and LSPR modes. A prominent resonant absorbance peak is produced in the visible range of light by metal nanoparticles in LSPR while the SPR involves no metal nanostructures and no such visible properties.

The best example of LSPR is the red colour of dispersed AuNPs in solution. Typically, when visible light is incident on AuNPs that are smaller than the incident wavelength of light, the surface electrons (6s conduction band electrons) of AuNP start to oscillate collectively with a resonant frequency that depends on the NP size and shape. ${ }^{46}$ The wavelength that they absorb is dependent on the size of the particles. Small AuNPs (e.g. 10-20 nm) absorb green light which corresponds to an absorption band at $520 \mathrm{~nm}$ in the visible range and thus the AuNPs solution appears red in colour. Similarly, when the AuNPs aggregate, their surface plasmons combine (broadening of plasmon band) to give an absorption band in the $620-780 \mathrm{~nm}$ range (red light). Thus, there is a band shift and the aggregated particle solution occurs purple/blue to naked eye. This predictable colour change forms the basis for absorption based colorimetric assays. ${ }^{47}$ The LSPR sensors can 
be distinguished into two broad categories: aggregation sensors and refractive index sensors. As the name suggests aggregation sensors involve drastic changes in the colour of a solution of nanoparticles due to plasmon coupling that is visible to the naked eye. On the other hand, the red shift induced by a change in the refractive index around metal nanostructures forms the basis of refractive index sensors. In this thesis colorimetric AuNP aggregation assays will be described in detail. $^{48}$

\subsubsection{Colorimetric biosensors}

Colorimetric biosensors basically involve a colour change proportional to the concentration of a measured substance (analyte), which can be visualized and quantified in most cases.

The plasmonic nanoparticles act as transducers to report a change in the refractive index which can occur as a result of any molecular binding event on its surface which causes a spectral shift or red shift visible to the naked eye. This way molecular binding can be monitored, and such a device is known as a colorimetric biosensor. Noble metal nanoparticles of silver and gold are the most widely used in the construction of plasmonic biosensors. Although silver exhibits sharper and more intense LSPR bands compared to gold, the higher chemical stability of gold nanoparticles favour its application for biosensing. Colorimetric biosensors are becoming attractive platforms for the detection of small molecule targets owing to their high sensitivity and specificity. They are highly attractive for point-of-care detection of several analytes ranging from viruses to protein biomarkers, whole cells and even small molecule analytes like drugs, hormones, and antibiotics. Compared to other biosensors which require expensive instrumentation and scientific expertise such as SPR, imaging ellipsometry, sandwich assays using chemically functionalized nanoparticles (NPs), they are simple and cost effective and provide visual detection with rapid reading. ${ }^{49}$

\subsubsection{AuNP aggregation assays}

One of the most widely implemented and effective nanomaterial for the construction of colorimetric biosensors is AuNPs. AuNP based biosensing assays are gaining considerable attention due to their simplicity and versatility. The assay takes advantage of LSPR i.e. the predictable colour change associated with the aggregation (from red to blue due to plasmon coupling) and dispersion (from blue to red due to localized surface plasmon resonance) of AuNPs 
in response to an addition of electrolyte (change in their external environment) ${ }^{50}$. Figure 1.5 clearly illustrates the various properties of AuNPs which make them a suitable candidate for colorimetric biosensing applications. ${ }^{50}$

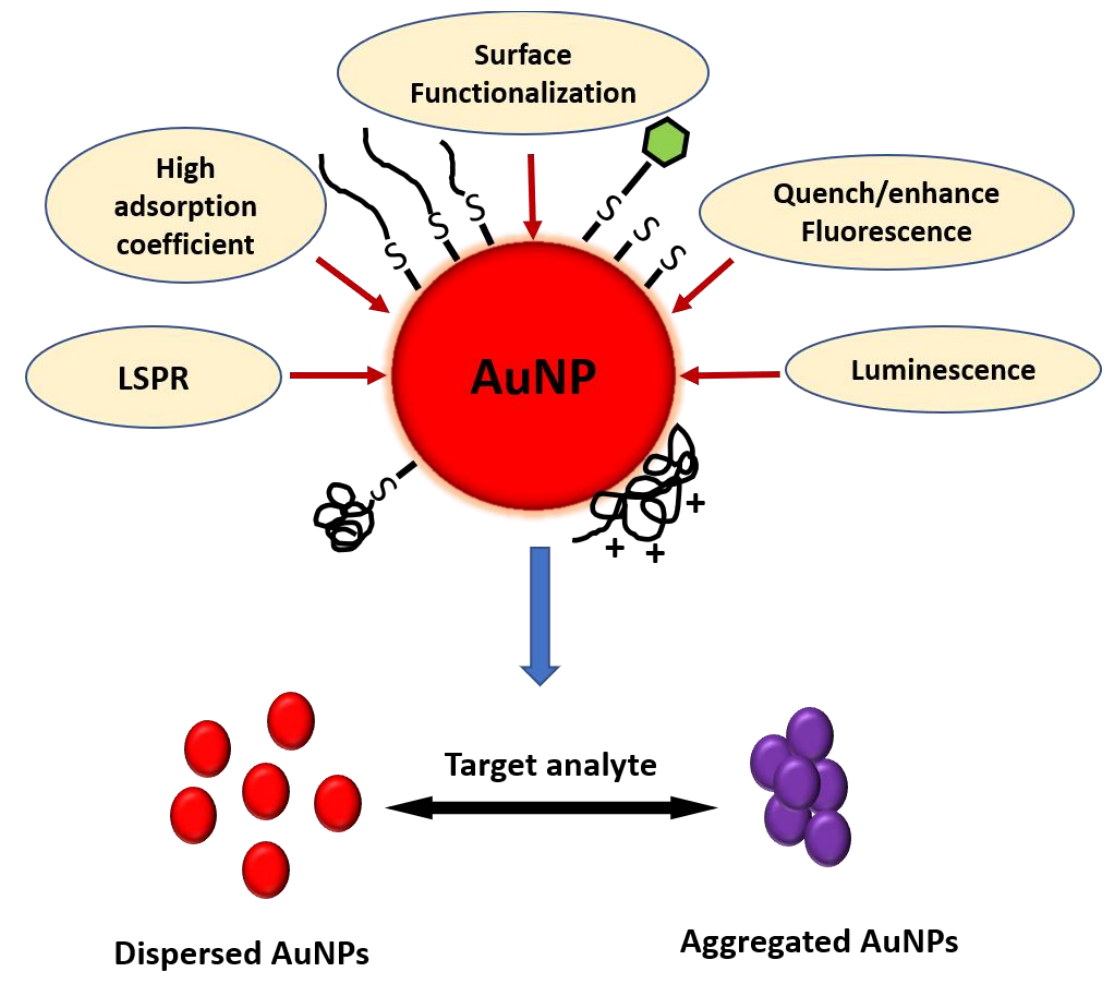

Figure 1.5 Physical and chemical properties of AuNPs and schematic illustration of the AuNPs based colorimetric (aggregation/dispersion) biosensing system. The unique properties of AuNPs such as their localized surface plasmon resonance, high adsorption capacity, surface functionalization possibilities, fluorescence quenching/enhancing properties, luminescence, conductivity etc make them a suitable choice for application into biosensing systems.

\subsubsection{Colloidal AuNPs stabilization: DLVO and non-DLVO forces}

The stability of colloidal AuNPs is of prime importance which needs to be considered before its application into various biomedical platforms. AuNPs in solution can be stabilized by introducing molecules that interact with AuNPs surface via electrostatic and/or binding interactions. For this purpose, AuNPs act as transducers that can be stabilized or functionalized with various sensing probes such as antibodies ${ }^{51}$, nucleic acid aptamers ${ }^{52}$ or chemical ligands. 
The phenomenon of stabilization of AuNPs in a well-defined and controlled environment has been generally explained by the DLVO (Derjaguin-Landau-Verwey-Overbeck) theory. The DLVO theory accounts for the stability as a balance between attractive van der Waals interaction and the repulsive electrostatic interactions. The theory says that the potential energy variations that occur when two particles approach each other are a result of the resultant net attraction and repulsive forces as a function of the interparticle distance as shown by equation 1.2. ${ }^{50}$

$$
\Delta G=\Delta G_{\text {van der Waals }}+\Delta G_{\text {electrostatic }}
$$

Where, the net interaction potential G between two AuNPs is determined by the van der Waals attraction $G_{\text {van der Waals }}$ and the electrostatic repulsion $G_{\text {electrostatic }}$ values.

Despite the existence of a considerable body of research which complies with the DLVO theory, it has long been contradicted and understood that the classical DLVO theory does not completely explain the results across different experimental conditions. For example, the model fails to predict based on DLVO theory when two particles approach closer than a few nanometers. In such instance several non-DLVO forces such as hydrophobic, steric and solvation forces come into play. Therefore, the classical DLVO theory can be modified to account for the new idea and the new equation 1.3 becomes,

$$
\Delta G=\Delta G_{\text {van der Waals }}+\Delta G_{\text {electrostatic }}+\Delta G_{\text {non-DLVO forces }}
$$

\subsubsection{Strategies for AuNPs colloidal stabilization}

AuNPs stabilization strategies can be broadly classified into three main ways, electrostatic, steric and electrosteric (a combination of both electrostatic and steric) mechanisms (Figure 1.6). In aqueous solutions bare uncharged AuNPs tend to aggregate because of van der Waals attractive forces. However, for applications to develop colorimetric biosensors, the strategies to achieve colloidal stabilization by introducing repulsive forces between particles in the absence of target analyte are of utmost importance. ${ }^{50}$ 
A

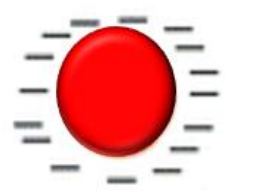

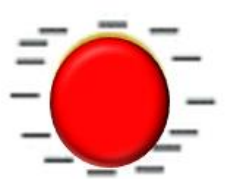

B

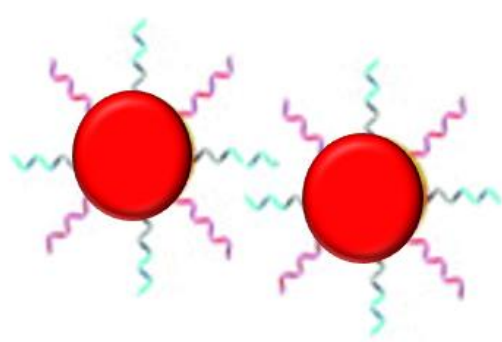

C

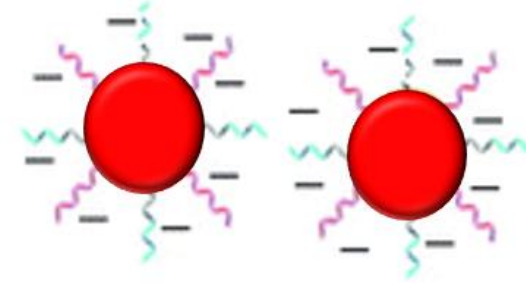

Figure 1.6 Schematic illustration of AuNPs colloidal stabilization A) electrostatic B) Steric C) combination of electrostatic and steric forces

Electrostatic: In the case of electrostatic stabilization, the particles carry similar electrical charge such that together with the counter ions in the medium they form a electrical double layer which helps to maintain the colloidal stability against van der Waals attractive forces. This assembly is highly sensitive to salt because the thickness of the electrical double layer is dependent on the bulk ionic strength if the solution. ${ }^{53}$

Steric: In case of steric stabilization, the physical barrier created by the bound or adsorbed molecule prevents the particles from crowding. The strength of steric stabilization is independent of ionic strength of the solution and depends largely on the capping density and molecular size. ${ }^{53}$

Both electrostatic and steric stabilization can be achieved by highly charged biomolecules such as nucleic acids, proteins, drugs etc.

\subsubsection{Mechanisms of AuNP aggregation}

The principle of colorimetric AuNPs aggregation assays involve the preparation of a stable colloid whose stability is disrupted by the addition of an analyte followed by a visible colour change. For successful aggregation of AuNPs, it is necessary that the distance between the particles is less than 2.5 times their individual diameters. ${ }^{50}$ The resulting aggregation induces the plasmon coupling of the AuNPs causing a red shift/broadening of the SPR band and the visual colour change from red 
to blue/purple. ${ }^{54}$ The mechanisms involved that lead to AuNPs aggregation can be classified into two broad categories: Interparticle cross linking aggregation and non-cross-linking aggregation

\section{Interparticle cross-linking aggregation}

In this mechanism the AuNPs are brought in contact with each other through linkages (via a cross linker) between the AuNPs (Figure 1.7). This occurs by using cross linkers that have two binding sites and can connect/link two AuNPs or this can occur via direct interaction without cross linkers. Specific binding forces such as H-bonding, electrostatic interaction, metal-ligand coordination etc. associated with biological recognition events such as DNA hybridization, DNA-ligand interactions, DNA cleavage etc. overcome the interparticle repulsive forces to cause aggregation. Examples of colorimetric assays based on cross-linking aggregation mechanism involve the monitoring of alkaline phosphatase (ALP) activity using Zinc to modulate the aggregation of AuNPs functionalized with zinc responsive polypeptide and many more. ${ }^{55}$

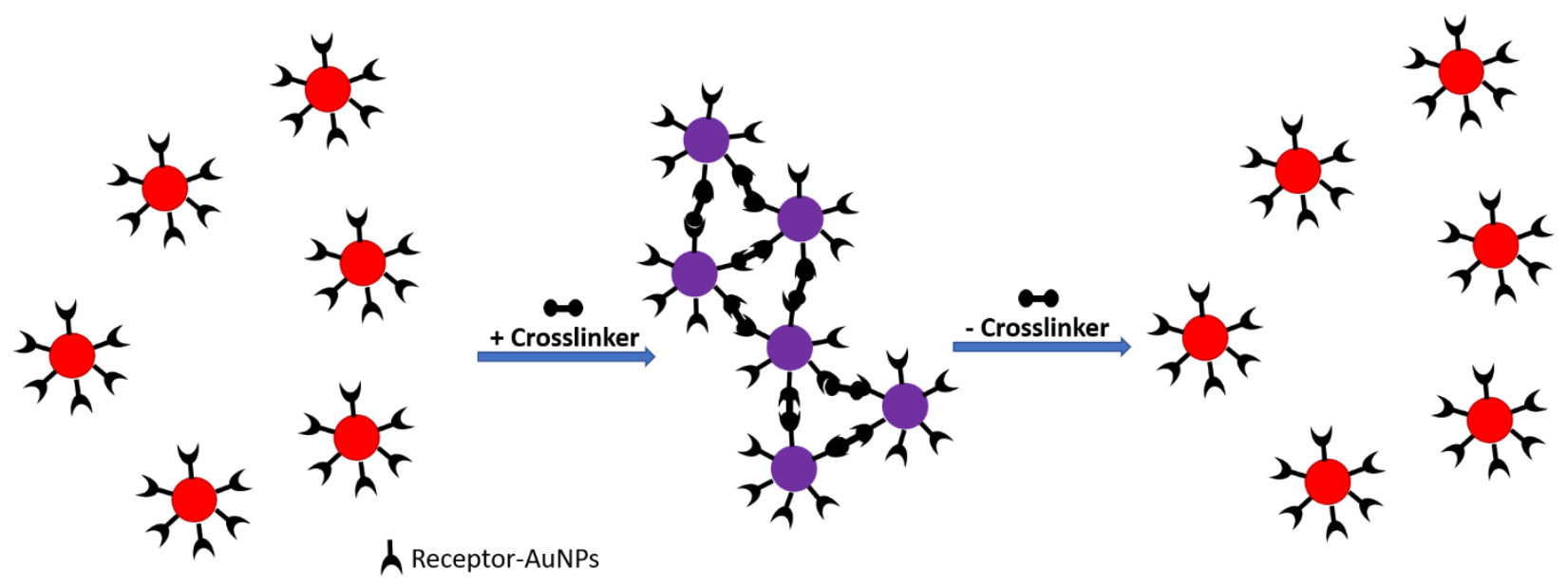

Figure 1.7 Schematic showing the basic principle of the interparticle cross-linking induced aggregation mechanism

Interparticle cross linking aggregation that occur without cross linkers can take place by employing two different AuNPs solutions functionalized with complementary ligands (Figure 1.8). Examples include DNA hybridization and antibody antigen reactions. 

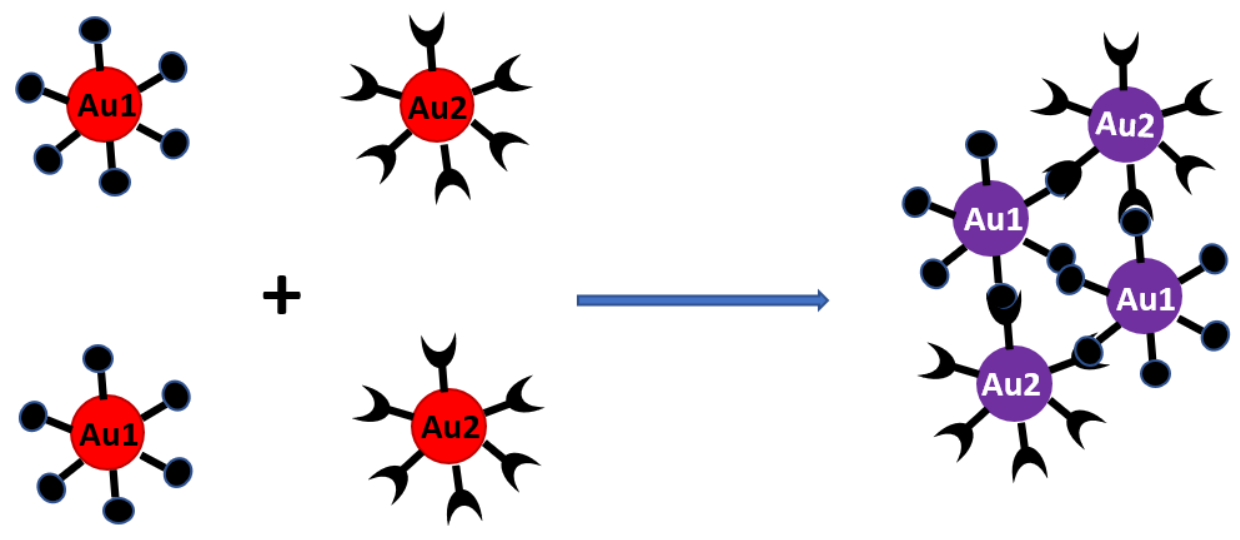

Figure 1.8 Schematic of interparticle cross-linking mechanism without an external crosslinker

This mechanism requires tedious modifications of ligands or targets to enable them to form interparticle bonds or cross-linking reactions. Mainly limited to cases wherein the targets or ligands have at least two binding sites. It is a time-consuming process and the reaction can be easily hampered if the probes have poorly accessible ligand sites.

\section{Non-cross-linking aggregation}

To overcome the shortcomings mentioned in case of cross-linking aggregation, non-cross-linking aggregation harnesses the ability of the AuNPs to interact with each other without actual bond formation (Figure 1.9). This is possible when there exists a strong interaction between the ligand and the analyte such that the interaction removes the adsorbed ligand from the AuNPs surface or induces a conformational change which alters the AuNPs resistance to salt induced aggregation. The strategy was pioneered by Sato and his colleagues to detect DNA polymorphisms. ${ }^{56,57}$ The idea was that when a target polynucleotide would be complimentary to the ligand in sequence and chain length, hybridization would occur leading to dsDNA formation on the surface of AuNPs. This would in turn alter the AuNPs tolerance to salt. 
A
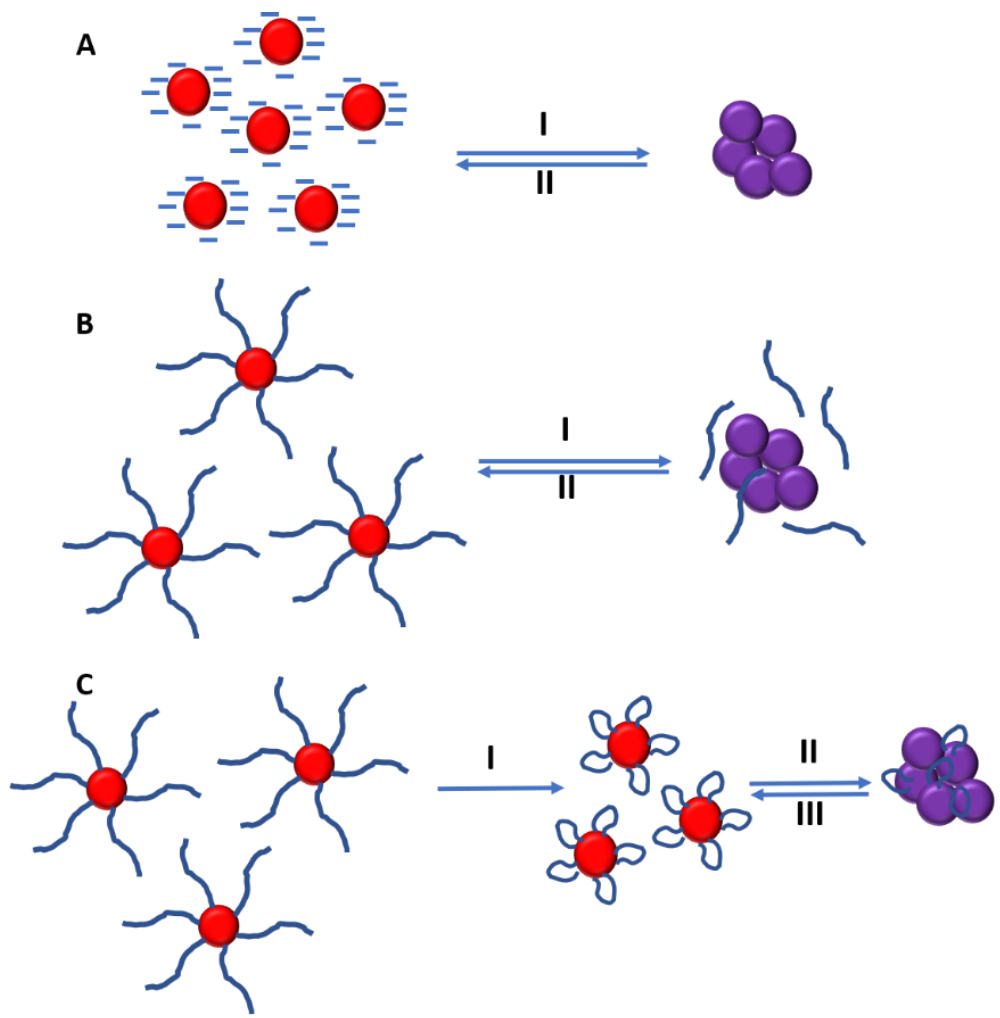

Figure 1.9 Schematic of non-cross-linking mechanisms: A) (I) Removal of electrostatic forces induce aggregation from red to blue (II) acquisition of repulsive forces dissociates aggregation. B) (I) Removal of steric or electrosteric forces induces aggregation and (II) vice versa C) (I) Aggregation induced by conformational control of the steric agent (II) finally leading to aggregation (III) Vice versa

Biosensing based on non-cross-linking colloidal aggregation offers one of the most simple, sensitive and label free assays that most commonly offer instant visual determination of the sensing event. Unlike interparticle cross-linking mechanism which is time consuming as well as require either a target analyte/receptor with multiple binding sites or a cross linker for inducing the reaction, non-cross-linking mechanism has various desirable attributes. However, high ionic strength solutions are frequently used in non-cross-linking mechanism and it is important to choose an appropriate solution and determine the nature of interference that the ionic strength solution may cause in order to obtain optimum assay performance.

Early experiments used AuNPs as sensing probes to detect hybridization interactions between DNA strands which caused colloidal aggregation followed by a visual colour change. The strategy involved mixing two AuNP colloid functionalized with non-complementary DNA strands followed by addition of a third DNA strand complimentary to both the existing strands such that 
the addition of the third strand induced immediate aggregation induced by the hybridization event and appearance of a drastic colour change from red to blue/purple. This event could be reversed by inducing dehybridization by either changing the ionic strength or raising the temperature above the melting point of DNA. ${ }^{48}$ Later on, Li and Rothberg extended the same strategy to detect DNA hybridization using unmodified gold nanoparticles. ${ }^{58,59}$ The detection strategy exploited the differential affinity of double stranded DNA (dsDNA) and single stranded DNA (ssDNA) for AuNPs. dsDNA finds it difficult to adsorb AuNPs due to electrostatic repulsion between negatively charged rigid phosphate backbone and AuNPs whereas ssDNA easily adsorbs onto AuNPs owing to their conformational flexibility and protects AuNPs against salt induced aggregation. Colloidal AuNPs samples that contain ssDNA appear red under a certain ionic strength which would lead to electrostatic screening and aggregation in their absence but colloids containing dsDNA are destabilized and turn purple. These assays have been reported to be sensitive enough to detect single base mismatches and have detection limits lower than 100 femtomoles.

AuNP aggregation assays are not restricted to DNA sensing rather protein sensing has also been demonstrated. Such reactions involving biomolecular interaction between antigen protein and antibodies are known as immunoreaction. For example, the detection of anti-protein A was successful via an immunoreaction between protein A functionalized AuNPs. ${ }^{60}$ Other examples include detection of concanavalin-A, a carbohydrate binding protein via mannose stabilized AuNPs. ${ }^{61}$

Application of AuNPs aggregation assays have also been extended to probe enzymatic activity. Examples include phosphorylation process of kinases, protein catabolism of proteases (thrombin and lethal factor), DNA cleavage by endonucleases etc. ${ }^{62,63}$ Apart from these applications, colorimetric aggregation assays can also be performed in complex biological media. For example, antibody-nanoshell conjugates have been used to detect immunoglobulin in saline serum and whole blood environments. However, application of such aggregation assay in a rather complicated body fluid such as real human saliva is yet to be reported.

Demonstration of AuNPs based label free colorimetric assay has been developed for the picomolar detection of thrombin in blood plasma. ${ }^{64}$ Unmodified AuNPs have been used to develop a label free colorimetric assay for detecting the adenosine deaminase activity by exploiting the strong 
interaction between amino group of adenosine and AuNPs. The assay was highly sensitive yielding a detection limit of $1.526 \mathrm{U} / \mathrm{L} .{ }^{65}$ Demonstration of successful AuNP aggregation assays for the detection of a wide variety of biologically relevant small molecules has been reported in literature. Oxytetracycline was detected with high sensitivity and specificity using aptasensors with a detection sensitivity of $25 \mathrm{nM}^{66}$ Similarly, 17ß-estradiol was detected using DNA oligonucleotides with detection levels of nanomolar in biological matrix (urine) and picomolar levels in buffer. ${ }^{67}$ Also, detection of streptomycin in raw milk was successfully demonstrated with an LOD of 60.2 nM. ${ }^{68}$

In this context, in the next section aptamers would be introduced briefly before transitioning into aptamer-based biosensors or aptasensors discussion.

\subsection{Aptamers}

Aptamers are short ssDNA or RNA nucleic acid sequences that are mostly synthetically derived (and hence also referred to as oligonucleotides) from a library of random nucleic acid sequences through an iterative process known as SELEX (Systematic evolution of ligands by exponential enrichment). Aptamers have the ability to fold into unique 3-dimensional structures and bind target molecules with high affinity and specificity (Figure 1.10).

Aptamers were descried for the first time in 1990 by A.D. Ellington and proposed to be superior to antibodies. ${ }^{69}$ Antibodies are proteins that are produced by the immune system in response to an infection. Therefore, they need to be extracted from a living organism after they have been induced to produce it.

The main advantages of aptamers over antibodies are:

- The specificity and affinity of aptamers can be tailored via easy chemical modifications

- Excellent stability in a range of ionic conditions, temperature and $\mathrm{pH}$

- Cost effectiveness and robustness due to easy chemical synthesis without involving live animals 
- Aptamers can be easily generated with high specificity and affinity against low molecular weight targets/targets which lack immunogenicity

- Least batch to batch variability

- Longer shelf life

- Relatively small size of aptamers enables their penetration into for example tumor cells easily

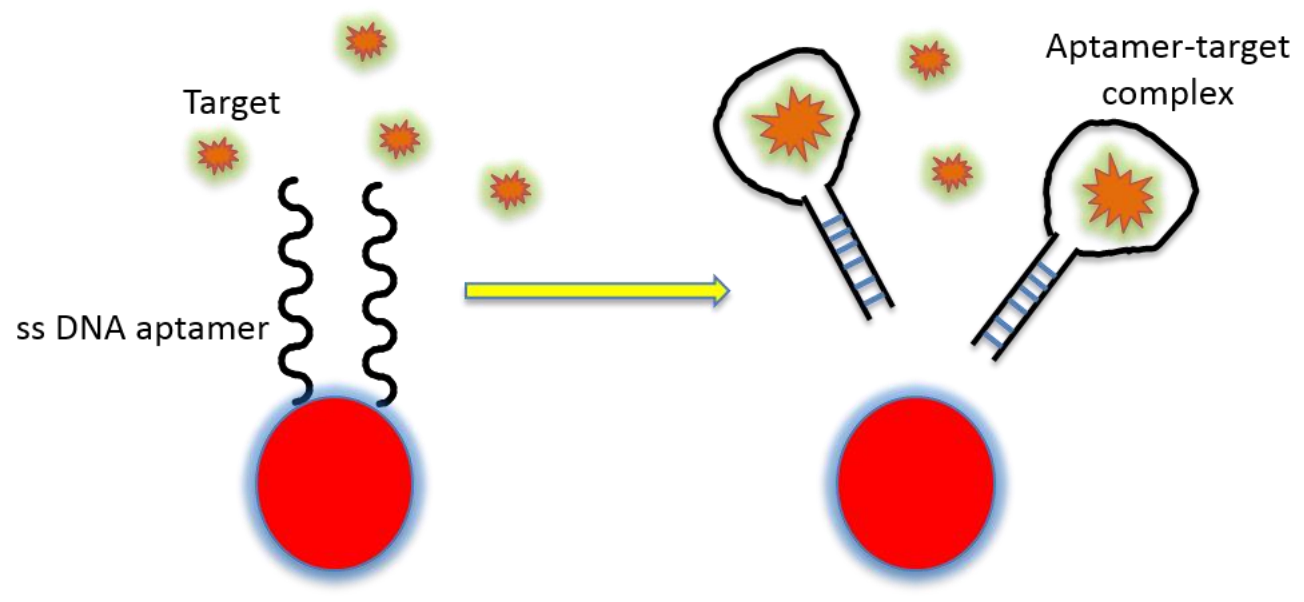

Figure 1.10 Schematic showing the unique conformational change undergone by aptamers induced by the target

Following the discovery of aptamers, they were extensively tested and used in a variety of applications. However, till date only a handful of aptamers have been commercialized in diagnostics and therapeutics. The most prominent (FDA approved) therapeutic aptamer that was commercialized in 2004 is Macugen by Pfizer. ${ }^{70}$ The Macugen is used to treat neovascular agerelated macular degeneration (AMD). Few other aptamers for the treatment of AMD and other renal diseases are in the advanced stages of their clinical trials. However, in case if diagnostics, numerous aptamers have been successfully developed and applied to diagnostic platforms but their translation into point-of-care (POC) is still in its inception. 
In this thesis, DNA aptamers have been used as a bioreceptor/probe to investigate the presence of methamphetamine in oral fluid using a colorimetric aptasensors system. However, before moving into the details, it is necessary to introduce the structure/building blocks of DNA aptamers and their selection procedure.

\section{Overview of DNA structure}

DNA or Deoxyribonucleic acid is a complex molecule which are the carriers of genetic information necessary for the structural and functional viability of an organism. Every multicellular organism has cells which contains the full set of DNAs necessary for the survival of that organism. During reproduction in organisms. A portion of each organism's DNA is passed into their offspring. Therefore, DNA ensures a level of continuity among generations while allowing for new combinations that contribute to the diversity of life.

All DNA is composed of smaller basic units known as nucleotides (Figure 1.11). Each nucleotide consists of three components, a nitrogenous base, a carbon-based sugar molecule and a phosphate group attached to the sugar molecule. Depending on the nitrogenous bases, nucleotides are primarily of four types namely, Adenine (A), Guanine (G), Cytosine (C) and Thymine (T). The deoxyribose sugar molecule is a 5-carbon ring shaped molecule wherein each carbon atom is referred to by a number from 1' to 5'. Of all the carbon atoms, 5 ' is the most notable because it is attached to the phosphate group and opposite to the 5' is the 3' which has a hydroxyl group attached to it. These are the carbon atoms which determine the binding functions of the nucleotides. Adjacent nucleotide molecules attach to each other via the 3' end of one molecule with the 5' end of another nucleotide forming a phosphodiester bond. Such joined nucleotides together are known as a polynucleotide. Such alternating sugar phosphate arrangement forms the backbone of the DNA. 


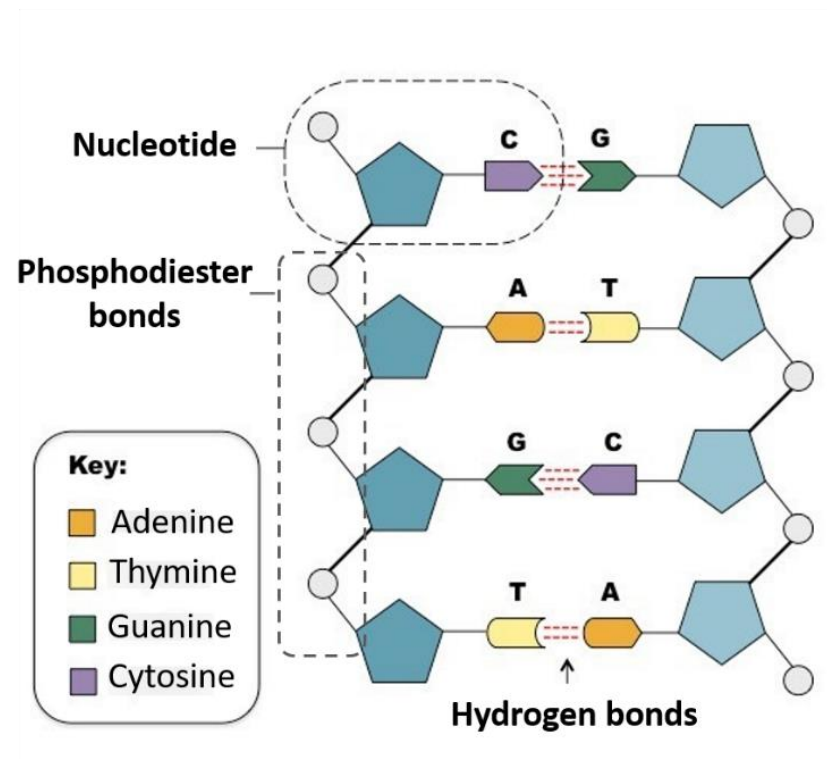

Antiparallel DNA strands

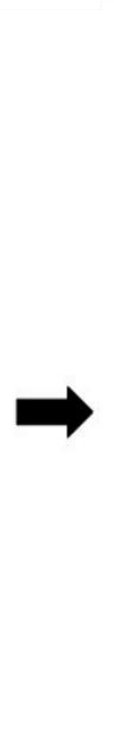

Sugar phosphate

backbones

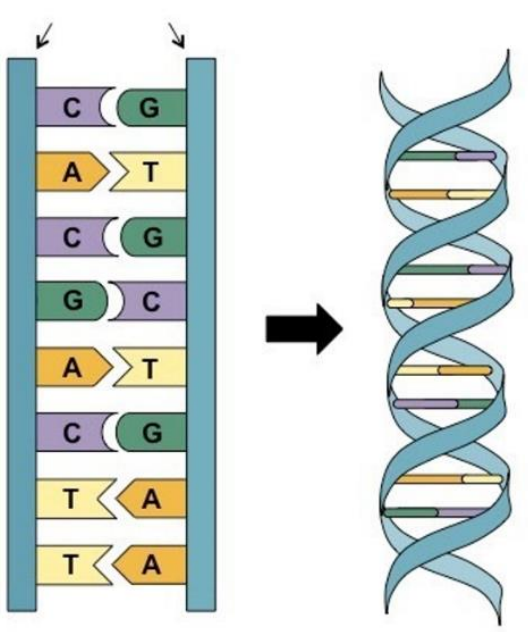

DNA ladder

Double helix

Figure 1.11 The schematic illustration of the basic structure of a DNA

DNA can often be found as a single stranded polynucleotide form, but it is most stable in its double stranded form. Double stranded DNA is formed when the nitrogen base of one DNA polynucleotide strand binds with the nitrogen base of another DNA strand via hydrogen bonds. These bonds are not random but are very specific in nature such that an Adenine bonds via two hydrogen bonds with thymine only and Guanine bonds via two three bonds with Cytosine only. These bonds help to form a double stranded DNA which looks like a ladder with sugar-phosphate side supports and base-pair rungs. The two polynucleotide chains that make up the DNA double strand run opposite to each other such that the sugar-phosphate ends are antiparallel. Apart from the ladderlike structure, another key characteristic of double stranded DNA is its unique threedimensional structure. In 1952 the first images of DNA molecule were captured in X-ray diffraction by scientist Dr. Rosalind Franklin, however, the details in the images were sparse but it was still interpreted that the nucleotides were arranged in a spiral shape known as a helix. Around the same time Watson and Crick were also investigating the definitive form of the stable structure of DNA inside cells nuclei. Watson and Crick used Franklin's images and with the support of their own evidence proposed that the DNA had a double helix structure which is a ladder-like structure that is twisted throughout its length. All evidences by Franklin, Watson and Crick were published in 1953 in Nature. ${ }^{71,72}$ 
The structural stability of the double stranded DNA is maintained by a combination of noncovalent interactions such as hydrogen bonds between nitrogenous bases, $\pi-\pi$ stacking between base pair and hydrophobic interactions between the water molecules and bases. DNA is highly negatively charged at neutral $\mathrm{pH}$ due to the phosphate backbone. At low $\mathrm{pH}$ the backbone is protonated affecting the structure of the DNA.

\subsubsection{Production of aptamers by SELEX}

The process of selecting an aptamer specifically for a target molecule is known as SELEX. The process was first discovered by Turk, Gold, Ellington and Szostak in 1990. ${ }^{73,52}$ Traditionally aptamers have been selected in-vitro but recent advances have enabled the selection in-vivo within cultured cells as well. In addition to the traditional protocol of SELEX many modified versions of selection and generation of aptamers in order to enhance affinity and specificity have been developed in the recent decade. The different modified SELEX include: Affinity Matrix SELEX, Capillary electrophoresis-SELEX, Capture-SELEX, Graphene oxide-SELEX, Gold nanoparticleSELEX. ${ }^{74,75,76}$ However, the fundamental idea of selection procedure of both DNA and RNA aptamers remain the same (Figure 1.12).

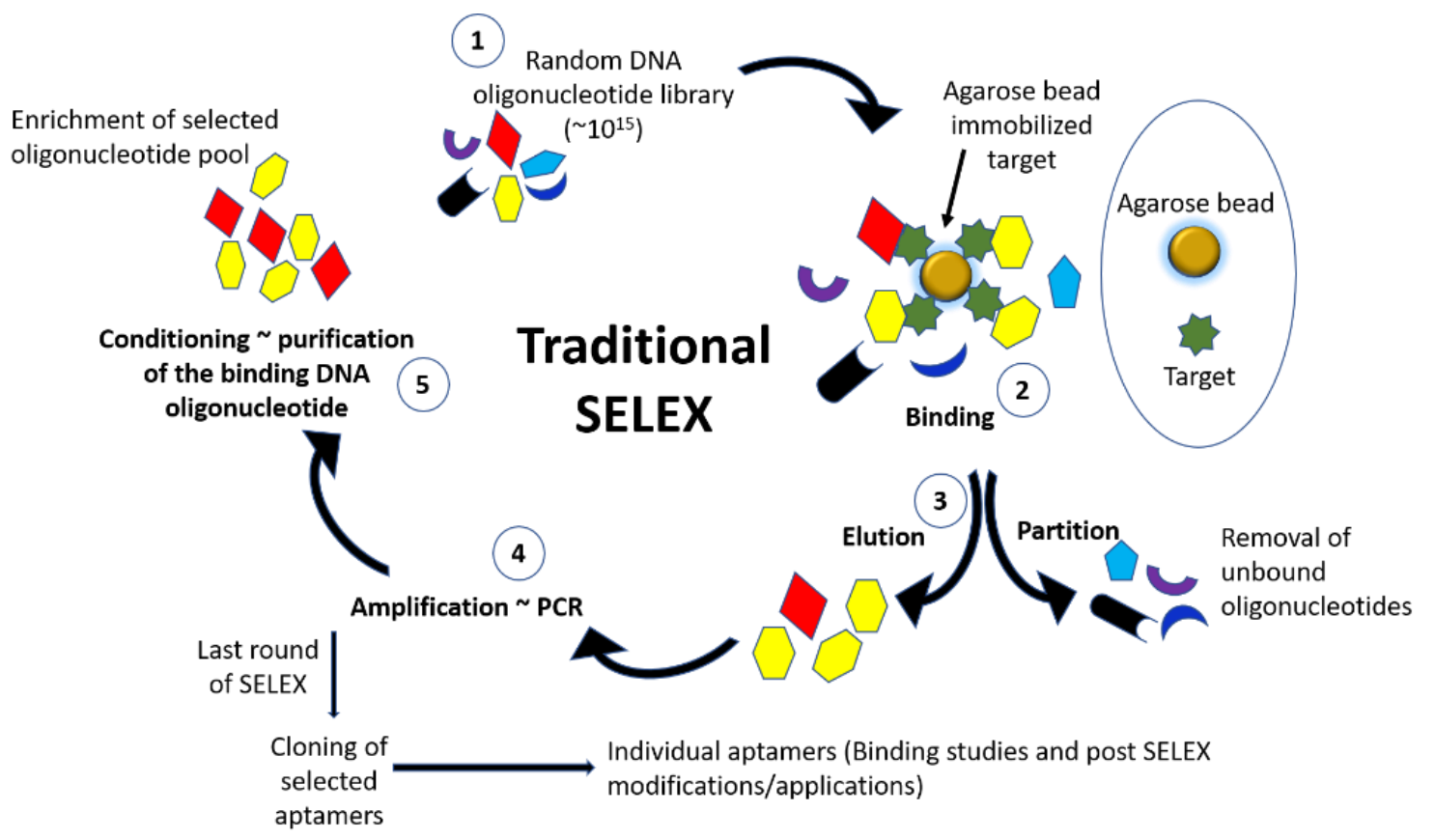

Figure 1.12 Schematic illustration of the traditional SELEX procedure that uses bead immobilized target for aptamer selection. 
The steps include chemical synthesis of a large pool of ssDNA library consisting of $10^{14}-10^{15}$ oligonucleotides. Generally, a ssDNA oligonucleotide consists of a core binding region which is essentially the binding region and this region differs in the nucleotide pattern in every oligonucleotide sequence to add diversity. This region also includes the supporting nucleotides which are part of the binding domain as they help the oligonucleotide to form binding conformations. At the ends of the sequence are primer regions whose nucleotide composition are known, and these are necessary for the PCR amplification step. Next steps involve, selecting ssDNA oligonucleotides based on their binding affinities for target wherein a library consisting of oligonucleotides is incubated with the target such that molecular recognition occurs via a combination of forces namely hydrophobic interactions, electrostatic interactions, hydrogen bonds and steric hindrances. Mostly the SELEX protocols differ from each other based on how the oligonucleotides are incubated with the target. For example, in the capture-SELEX developed by Stoltenberg et al. a docking sequence was inserted into the random regions of the oligos in the library such that these oligos could be captured/immobilized onto a magnetic bead that had a complimentary sequence to the docking sequence. This enabled the incubation with the target analyte free in solution and the corresponding release of the analyte binding oligos (on the basis of structural change induced by the target) from the beads. A lot of variations can be included such as incubating oligonucleotides with target free in solution rather than immobilized on beads etc. Next, the oligonucleotides (80-100 bp average size) with the strongest interactions are retained/ partitioned after washing off/eluting the weaker ones and amplified to form a second enriched pool. This iterative process of selection and enrichment is repeated until oligonucleotides (now called aptamers) with the strongest binding affinity are selected and isolated. This aptamer obtained after the upstream selection is comprised of the binding region, supporting nucleotides (which help in stabilizing aptamer secondary structure) and primer regions. Among these, the primer regions are completely redundant and nonessential and normally have no role in the binding process. ${ }^{77,78}$

Conventionally, 10-12 rounds of selection are required to obtain the enriched library consisting of aptamers with high affinity and specificity. The final enriched library consisting of target binding aptamers is sequenced to learn the exact nucleotide composition of the oligonucleotides. Next, the aptamers are validated post selection using standard characterization techniques such as pulldown assays, gel electrophoresis, Isothermal titration calorimetry or bio-layer interferometry. ${ }^{79-81}$ 
Nowadays, several other analytical approaches have been introduced along with SELEX to enhance aptamer selection procedure. These techniques include capillary electrophoresis, particle display technology and microfluidic chips. Moreover, since the traditional SELEX approach requires 10-12 iterated rounds of DNA amplification, this process becomes time consuming and more prone to errors. This is due to a reported high chance of PCR amplifying non-binders over the binders in $70 \%$ of attempts. ${ }^{82,83}$ As reported by Le et al. this issue could be addressed by one step ideal-filter capillary electrophoresis (IFCE) where the binders and non-binders move in opposite directions under the influence of electric field. ${ }^{84}$ The study concluded that the IFCE was significantly more efficient than the conventional SELEX in being faster, robust and having wider applicability. Similarly, other advancements in the SELEX procedure involve development of tissue SELEX, in-vivo SELEX, and 3D-cell SELEX. ${ }^{85,86,87}$ The recently developed SELEX method not only shortened the time from weeks to hours and from multiple selection rounds to as low as one selection round, the novel selection strategies also accounted for the aptamer modifications that may be required during application of aptamers as therapeutic carriers inside the body. The SELEX procedure is advancing at a fast pace and new generation SELEX are incorporated with these advancements to make them more dynamic and user friendly.

Most commonly, during SELEX selection of aptamers specificity selection is also included which ensures that the oligonucleotides that bind to other structurally similar molecules as target analyte are counter selected and removed so that the final selected aptamers have both high affinity as well as high specificity for the target analyte. This facilitates the aptamers selectivity performance for its target when applied to competing environments later.

Another important consideration when selecting aptamers using SELEX is the environment in which the SELEX is performed. Normally, the SELEX is carried out in a buffer that is specifically suited for the aptamer-target binding keeping in mind that the same buffer composition will be used during the application of aptamers in sensing systems. It is also important to select aptamers in complex media such as plasma, whole blood and saliva so as to ensure that the aptamer is able to remain functional and retain its target binding conformations in real complex samples. ${ }^{88}$

Post SELEX characterization of the aptamers involve affinity or equilibrium dissociation constant $\left(\mathrm{K}_{\mathrm{D}}\right)$ determination. This can be achieved by Isothermal titration calorimetry (ITC) along with additional information about the nature of binding and the thermodynamics of the interaction. A 
complete qualitative and qualitative characterization profile of the aptamer-target interaction post SELEX is necessary for designing suitable biosensors platforms. ${ }^{89}$

\subsection{Aptamer based biosensors or Aptasensors}

Biosensors using aptamers as BRE (Bio-recognition element) are known as aptasensors. Figure 1.13 highlights the general concept of an AuNP based aptasensors platform. In this section aptamer based AuNP aggregation assays will be discussed in detail with examples from literature regarding the advancement of this system.

Dispersed AuNPs
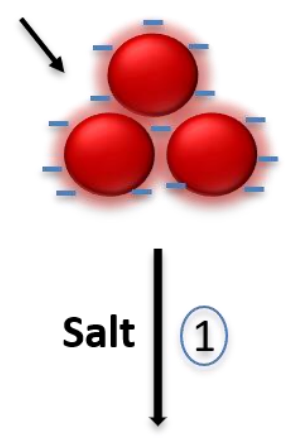

\section{Aptamers}
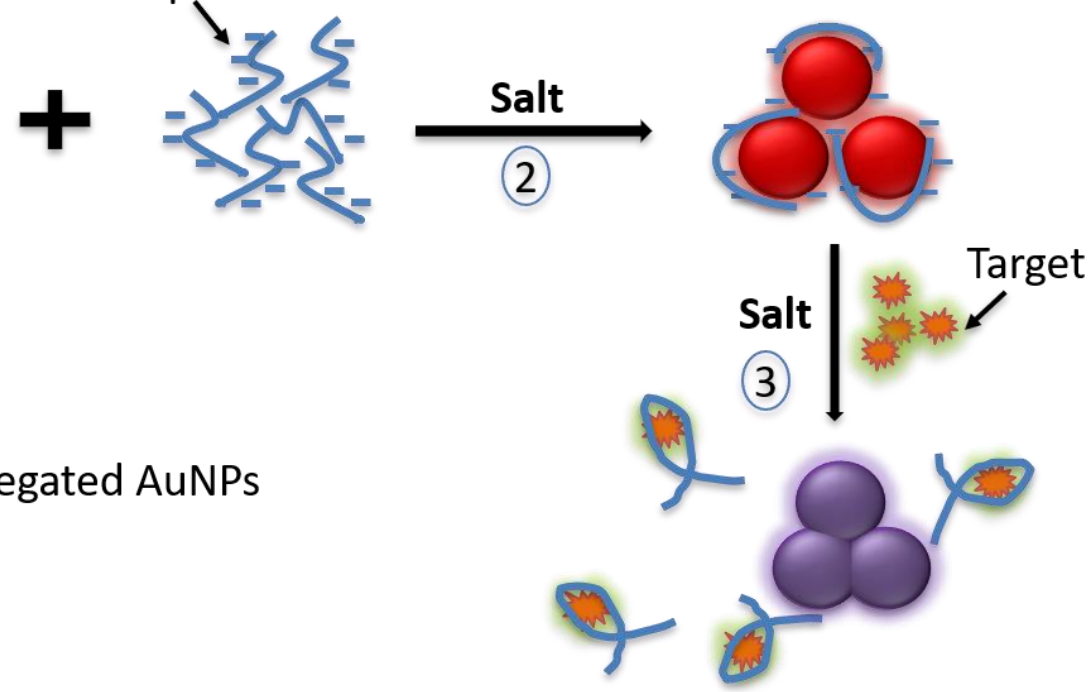

Figure 1.13 Schematic of an AuNPs based aptasensors for colorimetric detection of analytes. In the absence of aptamers AuNPs aggregate when subjected to certain ionic strength conditions (1). In the presence of aptamers, the AuNPs are protected against the ionic strength effect (2). Lastly, in such conditions when target is added, the aptamers preferably bind to the target leaving the AuNPs unprotected towards ionic strength effect (3).

Despite the small molecular weight of aptamers which range approximately around 5-30 kDa, aptamers are able to bind a variety of target molecules from proteins and peptides, bacteria/pathogens, whole cells including live cancer cells, toxins, viruses, tissues, hormones and other small molecules with high affinity and specificity. ${ }^{88}$ These nucleotide analogues of antibodies are increasingly gaining popularity in the research front as a potential alternative to the contemporary protein based probes. ${ }^{83}$ Owing to their high stability, low cost of production without 
involvement of animals, negligible batch to batch variations, ease of synthesis and labelling with minimal effect to their binding affinities and a predictable secondary structure, DNA aptamers have become the choice of researchers for application in biosensing and therapeutic platforms. ${ }^{90}$ These 'protein like' nucleic acids show a plethora of opportunities and researchers all over the world are engaged in exploring them.

Since the developement of the strategy to use unmodified AuNPs to detect DNA hybridization by $\mathrm{Li}$ and Rothberg, the strategy was later extended to detect aptamer targets basing on the assumption that target bound aptamers would not efficiently adsorb on AuNPs and free aptamers would adsorb on AuNPs and enhance their stability. ${ }^{91}$ DNA aptamers as probes in AuNP biosensing assays (aptasensors) are being considered a potential candidate for on-site detection of drugs for their high specificity and sensitivity. The key to designing colorimetric based biosensors is the control of the colloidal stability with an analyte of interest. ${ }^{47}$

AuNPs possess very high extinction coefficients and are very good candidates to signal that molecules are present in their surface or vicinity via distinct colour changes. DNA aptamers tend to adsorb to gold nanoparticles via physisorption due to the attractive interactions or metal coordination interactions occurring between the DNA nucleoside bases and AuNPs. These physisorbed aptamers on AuNPs form probes for AuNP-aptasensors, which are quite popular owing to their simplicity and low cost, and it has been exploited widely till date with nearing 1000 publications coming out every year recently. The lack of need for any time consuming and tedious chemical modifications makes this system particularly user-friendly. The interaction between gold and both ds and ssDNA is fine-tuned by a variety of factors such as electrostatic interactions, hydrophobic forces, affinity interactions between DNA bases and gold. ${ }^{92}$ However, the exact mechanism is a matter of scientific debate till date and not fully established.

Electrostatic interactions depend on the conformation adopted by DNA. For example, double stranded and highly folded structures would mostly have their phosphate backbone exposed thus carrying excess negative charge whereas ssDNA would have a more flexible conformation exposing either nitrogen bases or phosphate. ${ }^{58}$ This factor obviously results in different adsorption abilities of DNA strands to gold. DNA composition also plays an important role in determining the strength of interaction between DNA and gold. It has been proposed that DNA bases follow the following affinity trend towards AuNPs such that $\mathrm{A}>\mathrm{C}>=\mathrm{G}>\mathrm{T} \cdot{ }^{93}$ It is popularly believed that 
out of the four bases except Adenine, all three interact with gold via physisorption and adenine via chemisorption. The affinity of adenine towards gold is almost comparable to gold-thiol interactions. The dependence of DNA adsorption on gold is also dependent on the length of the DNA sequence. Shorter the length of aptamers greater is the adsorption on AuNPs. ${ }^{58}$ This is because short aptamers have a kinetic advantage over longer DNA sequences and result in faster adsorption, however, once adsorbed, longer aptamers offer better protection against salt induced aggregation than shorter aptamers.

Interestingly, when looking at ssDNA that exist as hairpin structures an unusual sequence length is preferred for adsorption as reported by He et al. ${ }^{94}$ The study showed that aptamers with 18-20 base pairs (bp) long hairpin (double stranded region) structure with sticky ends shorter than a certain length find it difficult to adsorb to AuNPs and result in lesser tolerance towards salt whereas as aptamers with a relatively short hairpin region ( $5 \mathrm{bp}$ ) and longer sticky end length are able to prevent AuNP aggregation. The adsorption increases with the increase in length of sticky end DNA of hairpin forms up to a certain range. This observation was owed to the effect of the double stranded hairpin region which affected adsorption of DNA with shorter sticky ends. This finding helped development of a AuNP colorimetric DNA assay with picomolar sensitivity and specificity and provided a new direction for AuNPs colorimetric assays utilizing aptamers with hairpin structures suggesting the effect of double stranded stem regions need to be accounted for when assuming faster adsorption of shorter DNA only on the basis of length. Nevertheless, the use of DNA aptamers in conjunction with unmodified AuNPs have been in unprecedented use for the development of successful colorimetric assays.

\subsubsection{AuNPs based aptasensors for small molecule detection}

Bare AuNPs are unstable in solution and a passivating layer of citrate or surfactants, proteins or DNA determine their colloidal stability along with the ionic strength, $\mathrm{pH}$ and temperature of solution. DNA aptamers adsorb to citrate stabilized AuNPs in solution by displacing loosely adsorbed citrate and confer a higher stability to the AuNPs against salt induced aggregation. This system is highly sensitive to the presence of an analyte of interest to which the aptamer has high affinity for. Depending on the affinity of the aptamer for the target, a conformational switch in the aptamer changes it from a ssDNA AuNP adsorbed state to a target binding three dimensional AuNP non-adsorbing state. This leaves the AuNPs susceptible to the increased ionic strength 
condition created and the aggregated AuNPs exhibit a blue/purple colour visible to the naked eye. This is the principle of a functional AuNPs colorimetric aptasensor. The extent of desorption and hence aggregation is directly proportional to the concentration of the target and this phenomenon is widely exploited in bio sensing. AuNPs diameter is an important factor and the range of size most exploited for these assays lay between 10-27 nm diameter. ${ }^{46}$

AuNPs based aggregation assays offer a promising platform for detection of small molecules. It provides a rapid and simple procedure to signal binding between aptamer and target. The most extensively studied small molecule aptamer with over 1000 reports in the past decade has been the ATP binding aptamer. Unmodified AuNPs have been used for the detection of ATP by exploiting the differential electrostatic interactions of dsDNA vs ssDNA. The mechanism has been illustrated in Figure 1.14. Using this simple strategy an LOD of $100 \mathrm{nM}$ was achieved. The assay was also successfully applied in diluted breast cancer cell lysates. ${ }^{95}$ Huo et.al. also reported ATP detection using unmodified AuNPs wherein the aptasensors was able to detect ATP as low as $50 \mathrm{nM}$ visually. ${ }^{96}$

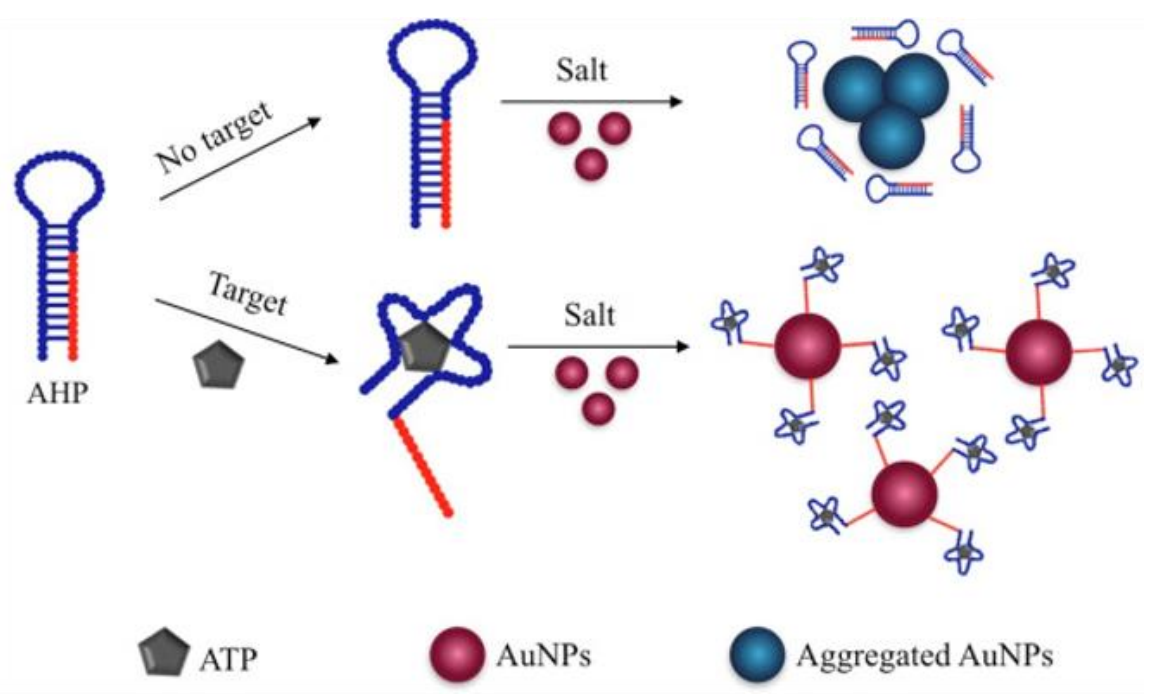

Figure 1.14 Mechanism of AuNPs aptasensors for ATP detection. The dsDNA aptamers in the absence of targets were unable to protect AuNPs from salt induced aggregation. However, in the presence of ATP, the dsDNA aptamer undergoes a conformational change to bind ATP and the free ss region of the ATP bound 
aptamer complex is able to protect AuNPs from salt induced aggregation. Figure reused with permission from publisher.

Derbyshire reported using an AuNP based assay to investigate cross-reactivity of three aminoglycoside binding RNA aptamers to eight different aminoglycosides. ${ }^{97}$ Similarly, AuNP aggregation assays have been used to assess binding of seven different small molecule binding aptamers. Antibiotics detection using AuNPs based apatasensor have been widely reported. Ampicillin (a penicillin-like antibiotic) used to treat different bacterial infections is used extensively in agriculture and medicine for the treatment of infections. An overdose of AMP could cause accumulation in foodstuff which may lead to serious complications. The first aptasensor using dual detection using both colorimetric and fluorescence methods was developed to detect AMP was by Song et al. ${ }^{98}$ The aptamer for AMP was selected via magnetic bead-based SELEX. Shayesteh and Ghavami reported the detection of AMP using a poly-A aptamer which was found to be a better substitute to thiol modified aptamers for the construction of aptasensor. ${ }^{99}$ Similarly aptasensors for kanamycin, another small molecule antibiotic was developed following the traditional AuNPs based aptasensor where a high affinity kanamycin binding aptamer was selected using affinity chromatography SELEX for the detection of kanamycin which interacted specifically with the GG region of the aptamer. ${ }^{33}$ Later, Sharma et al. developed a novel 'turnon/turn-off' strategy which combined ssDNA aptamers with tyrosine reduced AuNPs to form a peroxidase nanozyme for the detection of Kanamycin. ${ }^{100}$ This strategy eliminated the need for sodium chloride addition and resulted in a rapid one-step detection within 3-8 minutes with 15 folds enhanced detection sensitivity than conventional aptasensor approaches.

Multiplexing is a major advantage that aptasensors offer over antibody-based ELISA. Niu et al. exploited the multiplexing capacity of AuNP based aptasensors and detected more than one antibiotic simultaneously. ${ }^{101}$ The principle of the multiplex aptasensor reported by Niu et al. functions such that the presence of any one of the three targets would cause the corresponding aptamer to desorb from the AuNPs and a colour change would be observed. This technique however fails to discriminate or determine the exact target that is present. However, it could still be used in circumstances where screening of more than one analytes in a sample may be required.

The point-of-care or on-site application of aptasensors has been reported by Ha et al. for the detection of kanamycin. ${ }^{102}$ A label free nitrocellulose membrane paper chip-based method for the 
visual detection of kanamycin was developed which could also be extended to extract quantitative information by image analysis software. Similarly, a lateral flow assay for kanamycin was developed by Liu et al. using AuNPs-aptamer as probes, silver nanoparticles as signal amplification element along with biotin streptavidin chemistry for a highly stable and specific aptasensor. $^{103}$

Kanamycin has also been detected using an aptasensor in milk. The detection strategy involved an aptamer functionalized AuNPs which turned purple on the addition of kanamycin binding aptamer because of the kanamycin binding aptamer hybridizing with the complementary strand functionalized AuNPs. ${ }^{104}$ On the addition of milk samples containing kanamycin, the solution turned red due to disaggregation caused by the dehybridization of kanamycin binding aptamer which competitively bound to kanamycin. Another strip based portable detection strategy which involved construction of a novel cellulose nanofibrous membrane grafted with glutamic acid into cellulose acetate (G-CA NFMs), to which kanamycin binding aptamer was attached was developed by Abedalwafa et al. ${ }^{105}$ A complimentary strand functionalized AuNPs were used to complete the novel assembly via DNA hybridization. The addition of kanamycin disassembled the signal probe by replacing the cDNA. The resulting colour change was monitored, and this method gave excellent performance in milk and drinking water. Kanamycin detection in the complex matrix of serum has also been reported by Shayesteh and khsroshahi wherein, the specific interactions between cationic polymer PDDA, kanamycin and kanamycin binding aptamer were exploited to control the stability of AuNPs. ${ }^{106}$ AuNPs are stable with aptamer combined PDDA on their surface but in the presence of kanamycin, the aptamer desorbed and the solution aggregated. This method has been used to detect kanamycin in serum clinically. Several other antibiotic drugs such as tobramycin, streptomycin, neomycin, daunomycin, chloramphenicol, tetracycline, oxytetracycline, etc. have been detected extensively using AuNP based aptasensors. Moreover, apart from antibiotics heavy metals such as arsenic and mercury have also been detected using aptasensors. ${ }^{107,108}$

The major knowledge gap that exists in all the above discussed works is the extensive study of interactions between these small molecules and AuNPs apart from the aptamer. It is pertinent to investigate this interaction which may be dominant and may have been the reason for the colorimetric signals, but these control studies were missing in the above discussed works. Liu et 
al. has reported extensively about the interaction between several small molecule targets such as adenosine, dopamine, melamine, kanamycin, chloramphenicol, arsenic and mercury with AuNPs which were found to be dominating and interfered with the functioning of AuNPs aggregation assays. ${ }^{109-114}$ Through the studies it was possible for the group to prove that in most cases there was no binding between the aptamer and target rather, the dominant effect of small molecule target on AuNPs produced the observed colorimetric signals which were erroneously being interpreted as aptamer-target binding signals. Therefore, it becomes very important to properly investigate the mechanism of interaction of the target with AuNPs as the most important control in order to substantiate binding claims between aptamer and target. ${ }^{115}$

Moreover, it is also necessary to evaluate the nature of the interaction kinetics between the aptamer and AuNPs. Reports of tight binding interactions between aptamer and AuNPs have been reported by Liu et.al. who determine that around $2 \%$ of aptamers are able to desorb from AuNPs after adsorption and target introduction could not reverse the tight adsorption leading to complete sensor failure. ${ }^{116}$ Although the phenomena cannot be generalized but it does highlight the unsuitability of AuNPs-aptamer adsorption based assays especially when applied to small molecule detection whose likelihood of inducing a significant conformational change is quite limited.

\subsubsection{Colorimetric aptasensors for drugs of abuse detection}

The first colorimetric aptasensor was reported in 2002 for cocaine (a plant-based drug) with a limit of detection LOD $2 \mu \mathrm{M}$ by Stojanovic and Landry. The colorimetric assay was based on the principle of dye replacement strategy wherein a chromogenic dye complexed with a cocaine binding aptamer is replaced by the analyte causing an immediate change in the visible spectrum followed by precipitation of the dye. The work was motivated to overcome the sensitivity and complexity issues observed with previously reported fluorescent aptasensors for cocaine. The colorimetric assays offer simple preparation combined with rapid transduction. ${ }^{117}$ Later on, visual detection of methamphetamine using AuNPs based colorimetric aptasensor was reported by Yarbakht and Nikkhah ${ }^{118}$ in 2015 with an LOD of $5 \mathrm{mM}$ and by Q. Shi et al. around the same time also reported the visual detection of methamphetamine in urine with an LOD of $0.82 \mu \mathrm{M}$ using unmodified AuNPs assay. ${ }^{119}$ In order to achieve higher sensitivity and stability, Mao et.al. developed a novel nanostructure consisting of gold nanomaterial coated with a silver Ag core shell to form Au@Ag nanoparticles. ${ }^{120}$ The principle of detection is illustrated in Figure 1.15. A non- 
aggregation strategy was followed to detect methamphetamine and cocaine wherein, the Au@Ag nanostructure was modified with a reporter DNA sequence to form the reporter probe and a magnetic bead functionalized with capture DNA sequence acted as the capture probe. Both these probes were complimentary to a specific part of the target binding DNA and together in the absence of target, the aptamer facilitated the formation of a sandwich assembly. This sandwich complex formed could be removed from solution with the help of an external magnetic field and its removal would reduce the absorbance intensity of Au@Ag NPs. However in the presence of target, the sandwich complex could not be formed due to the non-availability of free aptamer and therefore the concentration of the $\mathrm{Au} @ \mathrm{Ag}$ analysed in the supernatant (after removal of sandwich complexes) would be directly proportional to the concentration of target such that more targets, more free Au@Ag NPs in solution which cannot be removed by a magnet (because of no sandwich complex formation).

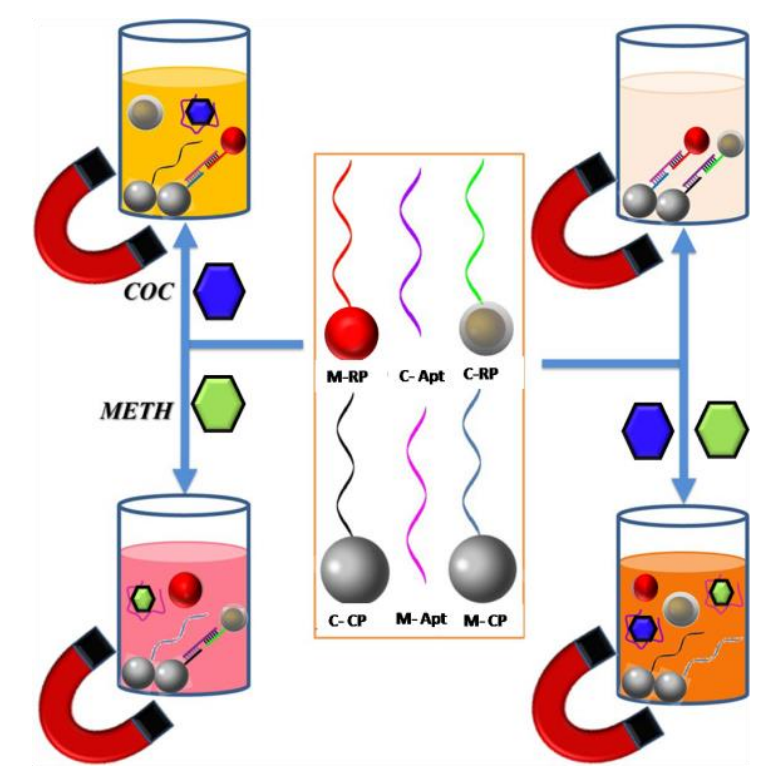

Figure 1.15 Multiplexed colorimetric detection of cocaine and meth by a novel aptasensor strategy using AuNPs and Au@Ag based nanostructures developed by Yang et.al. The image has been modified and adapted from the publication. ${ }^{120}$

Therefore, the absorbance intensity would increase in the presence of target and this could also be visualized as the presence of target and hence more Au@Ag in solution would exhibit deep yellow colouration. The system was used for multiplexed detection of cocaine and meth simultaneously by adding AuNPs as another reporter probe. In case of multiplex detection, the problem of 
overlapping spectra in the supernatant was solved by using an automatic non-negative matrix factorization (NMF) analysis. The LOD achieved for methamphetamine was $0.1 \mathrm{nM}$ and for cocaine $0.5 \mathrm{nM}$. The system also stable and functional in environmental matrices such as wastewater. The study concluded that the stability of the Au@Ag was so high that there was no problem of aggregation induced sedimentation which is a problem in the traditional AuNP aggregation assays.

More recently, cocaine has been detected with a detection limit of $0.97 \mathrm{nM}$ using simple unmodified AuNPs based colorimetric aptasensors strategy by Sanli et al. ${ }^{121}$ The sensor response was also validated in synthetic saliva using bovine serum albumin to mimic the biological aspect of actual oral fluid and it was found that the response of the sensor in synthetic saliva was suppressed by $64.5 \%$. The study did not report detection in real samples which would have helped in the evaluation of the performance of the aptasensors in saliva.

Apart from nanomaterial based colorimetric aptasensors, DNAzyme has been used for colorimetric biosensor for detection of methamphetamine. For example a label free DNAzyme based colorimetric hairpin aptasensor was developed by Nie et.al (2013) for the detection of cocaine with an LOD of $10 \mu \mathrm{M} .^{122}$ Lodha et.al. reported the detection of codeine using the strong coordination ability of codeine sulphate with AuNPs by displacing weakly bound citrate and finally crosslinking AuNPs. The detection was possible in less than 5 minutes with a LOD of $0.9 \mu \mathrm{M} .{ }^{123}$ The strategy (Figure 1.16) developed by Mao et.al. (2017) is based on the principle that the G-quadruplex hemin DNAzyme molecular beacon (DNAzyme MB) loses its peroxidase activity when it hybridizes with meth binding aptamer, however in the presence of meth, the aptamer dissociates from the DNAzyme MB and binds meth preferably. 


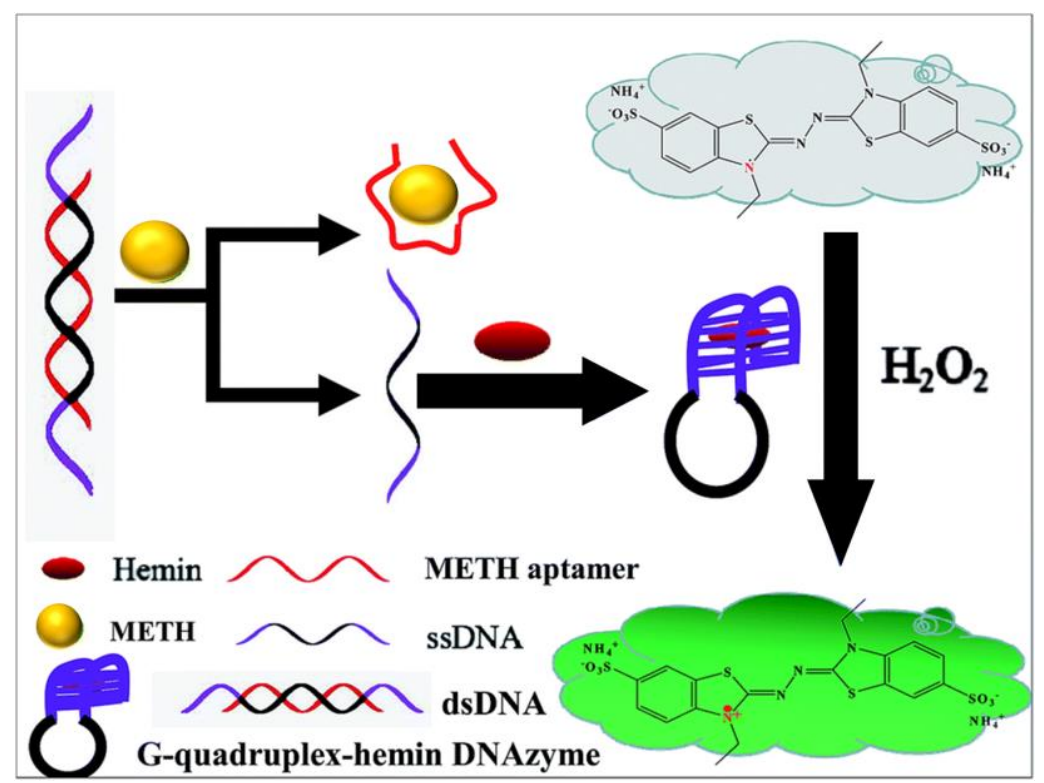

Figure 1.16 Colorimetric DNAzyme based aptasensors for the detection of meth developed by Mao et.al. The image has been adapted and modified from the publication. ${ }^{124}$

Then the DNAzyme regains its peroxidase activity and catalyzes the oxidation reaction involving a colorimetric substrate by $\mathrm{H}_{2} \mathrm{O}_{2}$. The colorimetric signal is then converted to measurable absorbance values. The LOD reported was $0.5 \mathrm{nM}$ in aqueous solutions. The system was reported to be promising for detection of methamphetamine in urine as well.

\subsection{Quantifying colorimetric aptasensors}

In the studies discussed above, a common need for quantitative analysis of colorimetric signals always requires a UV-Vis spectrophotometer. This is especially necessary for small molecule aptasensors which often suffer from less intense visual discrimination between the blank and test sample. Therefore, it becomes necessary for a spectroscopic analysis to determine and confirm the signal. A UV-Vis spectrophotometer is not portable which may limit the on-site application or determination of quantitative data. In all of the cited examples of colorimetric aptasensors, the use of a spectrophotometer has been mandatory. This shortcoming needs to be addressed in order to extract reliable quantitative data from visual signals. Therefore, it is necessary to think about portable detection systems which can offer sensitive quantitative analysis on site. For example, a low cost portable 3-D printed device to detect 8-oxo-dG in urine samples was developed with detection sensitivities comparable to the standard UV-Vis spectrophotometer. ${ }^{125}$ Smartphone 
based readout systems for aiding quantitative analysis is also a much-exploited area for AuNPs based colorimetric assays. For example, a smartphone-based readout system for a AuNPs based lateral flow assay was developed by Ruppert et.al. ${ }^{126}$ The authors used image processing to analyze the visual colorimetric signals obtained from the immunosensor. Other reports of such colorimetric immunosensors corroborated with image analysis for quantifying signals have been reported extensively. Few reports regarding AuNPs based aptasensors associated with image processing exists for example a recent report on detecting gentamycin using a colorimetric aptasensor was developed by Ramalingam et.al. wherein camera-based image processing as well as spectroscopic techniques were performed to evaluate it's POC application. ${ }^{127}$

\subsection{Challenges of small molecule detection with aggregation based AuNPs aptasensors}

Small molecules have been one of the most extensively studied aptamer targets. However, in the past decade, new aptamer development for SMs has been slow and this is indicative of several challenges associated with the success of SM binding aptamers. Major challenges of AuNPs aptasensors in general and for small molecules are:

- Small molecules present a unique challenge owing to their small size. This means that there are normally no more than one functional group which limits its recognition possibilities making detection challenging. It has been reported that the molecular weight of SMs is proportional to the affinity of aptamer (in most cases).

- Lack of specificity owing to the lack of suitable functional groups which often results in no hydrogen bond formation during binding to aptamer. Hydrogen bond formation is essential for specific recognition and binding. Therefore, fewer functional groups leads to low specificity.

- Due to the above-mentioned problems, low nanomolar levels of detection for SMs becomes very challenging to achieve as it requires high affinity and specificity

- Aggregation in AuNP assays can be non-specifically triggered due to a lot of other factors and not necessarily due to aptamer-target binding 
- SMs are prone to interact with AuNPs owing to their kinetic advantage over aptamers leading to total sensor failures in many cases

- The application of AuNP aggregation sensors to environmental matrices or biological matrices can be very challenging and require extensive sample pre-treatment procedures. Moreover, owing to the sensitive nature of the assays, it may be difficult resisting aggregation triggered by complex matrices even after pretreatment.

\subsection{Objectives, research questions and knowledge gaps}

The main objective of the thesis was the development and optimization of a colorimetric assay for the detection of methamphetamine in human saliva at cut-off levels of methamphetamine normally expected to be found in saliva for proving impairment ( $25 \mathrm{ng} / \mathrm{mL})$, using a family of methamphetamine aptamers selected by Auramer Bio. For the detection of meth in saliva, the saliva pre-treatment strategies needed to be designed keeping in mind the POC application of the sensor in future. Another objective of this thesis was to compare the effect of aptamer selection strategies on the performance of the sensor. Therefore, new generation SELEX selected aptamers were tested to evaluate that. Finally, a portable detection system was designed and investigated to meet the final objective of this thesis.

Regarding the discussion so far, it is necessary now to point out the knowledge gaps that exist while alluding to some potential problems that these aptasensor assays are facing. The main question that we must ask ourselves while developing colorimetric aptasensors is 'do aptamers always bind?' It is important to evaluate the binding interaction between aptamer and target via various control experiments to claim actual binding between aptamer and target. One of the major limitations with colorimetric aggregation aptasensor is the likelihood of getting misled by a visual colour change signal which may not always arise from aptamer target binding. Therefore, it is also necessary to evaluate the binding via independent techniques. In this thesis the problem of nonspecific interaction and artefacts have been strictly addressed to ensure the credibility of the colorimetric signals. 


\section{Do aptamer-AuNPs aggregation assays take place under a dynamic equilibrium?}

While the basis of the aggregation assay is assumed to be occurring under a competitive equilibrium between the aptamer-AuNPs and aptamer-target, it is important to investigate whether these interactions occur in such a straightforward manner. This is of high importance because it determines the sensitivity of the detection and puts a question regarding the suitability of these assays for small molecule detection. As per the assay principle the analyte induces a characteristic conformational change in the aptamer such that it leaves the AuNPs to bind to the target, however, in case of small molecules it is understandable that the conformational change may not be as significant due to various reasons, and the aptamer may remain trapped on the surface of AuNPs falling into a thermodynamic stability because of the activation energy to form binding state is very high. This may be possible in many cases and is a less researched area. In this thesis the adsorption kinetics of the aptamer AuNPs has been studied in Chapter 3 to reveal the time dependent adsorption and inactivation of aptamer structures on AuNPs such that the target binding capability of aptamers is compromised.

\section{Can these assays be applied to complex matrix of whole saliva without pre-treatment?}

AuNP aggregation assays are attractive platforms for their rapid sensing capabilities and high sensitivity. However, AuNPs are well known hotspots for biomolecule adsorption especially proteins which are mostly involved in tight interactions with AuNPs to form hard corona around it. Therefore, the application of these assays in whole biofluids such as saliva may be tricky due to a high possibility of proteins fouling the sensor surface. This thesis will address the challenging task of applying the sensor to the complex of matrix of saliva for the detection of meth in Chapter 5.

\section{What may be the effect of selection strategies on the aptamer in AuNP aggregation assay?}

The importance of selection strategies on the aptamer performance in a sensor which involves the aptamer interactions with a transducer before interacting with analyte may have a huge impact on the aptamer's recognition performance. Also, whether the target is immobilized or free in solution during selection stages of aptamer play a huge role in determining the aptamer's recognition capabilities in the downstream sensing platform. Recently, few studies have shown the enhanced performance of aptamers in sensing assays which were selected using modified selection strategies 
to closely match the sensor conditions. However, studies directly comparing aptamers selected using different selection strategies for the same target has not been reported yet. This thesis compares the sensing performance of two different aptamers selected via two independent SELEX strategies to investigate the actual effect of the SELEX strategy on the behaviour of the aptamer in Chapter 4.

\section{Do these assays hold promise for on-site detection?}

AuNPs aggregation assays are especially attractive platforms due to their translatability into onsite detection systems easily. While lateral flow assays have been quite popular as a translational platform from these aggregation assays, they are only reliable when the colour change is significant and offer a qualitative detection. However, for small molecules such as methamphetamine, the colour change may not be always obvious to the naked eye, necessitating more sensitive detection systems which can offer semi quantitative/quantitative measurements without the need for a dramatic colour change. In Chapter 6 of this thesis, the fabrication of a cost-effective light meter device has been demonstrated to facilitate on-site quantification of aptasensors response. 


\section{CHAPTER 2}

\section{General methodology}




\section{Chapter overview}

This chapter introduces briefly, the general principle of each technique and instrumentation used in the thesis. Each technique is described in the context of the AuNP aggregation assay, AuNPaptamer and protein interactions and aptamer characterization. The chapter begins with a description of the principles of UV-Vis absorption spectroscopy and its use to determine the analyte concentration in a AuNP assay. Circular Dichroism is described in terms of a characterization technique used to monitor aptamer structural changes in the presence of target. Next, DLS and zeta potential techniques are discussed with regard to their use in determining size and surface charge of AuNPs in the presence of different biomolecules. Finally, ITC is described as an independent qualitative technique to support the binding between aptamer and target. 


\subsection{UV-Vis absorption spectroscopy}

UV-Visible absorption spectroscopy is based on the interaction of matter with light. When light of a certain wavelength interacts with particles in solution, the electrons of the particles can be excited which enables them to jump from the ground state to an excited state by absorbing the incident light energy. The energy difference between the ground state and excited state levels of the electron is equal to the energy of visible or ultra-violet radiation absorbed or in other words, the energy difference corresponds to a particular absorption band/wavelength. The absorption bands are indicative of the molecular structure of the particles in a sample and a shift in the wavelength and intensity reflects the interactions between the molecule and its environmental constituents. The relationship between energy difference and wavelength is given by the Planck equation: ${ }^{128}$

$$
E=h v=\frac{h c}{\lambda}
$$

Where $\mathrm{E}=$ energy required to be absorbed by an electron to move from ground state to excited state, $\mathrm{h}=$ Planck's constant, $v=$ Frequency or wave number, $\mathrm{c}=$ speed of light, $\lambda=$ wavelength of incident light

From this equation it follows that the lower the energy required/ absorbed to excite the electrons, longer the wavelength of the absorption band.

$\mathrm{UV}$-Vis spectroscopy is also commonly used to quantify the concentration of an analyte. This is possible using Lambert-Beer's law which states how light is attenuated by the material it is passing through, more the number of absorbing molecules, greater the absorption value. The law states that the absorbance and hence the transmittance of a solution is directly proportional to the concentration of the absorbing species in the solution and the path length. ${ }^{129}$

The transmittance of a sample can be defined as a fraction of intensity of light transmitted over intensity of light incident i.e.

$$
T=\frac{I}{I_{0}}
$$

The relationship between absorbance and transmittance is logarithmic and can be defined as: 


$$
A=\log _{10} \frac{I_{0}}{I}
$$

Or

$$
A=-\log _{10} T
$$

According to Beers law:

$$
A=\log _{10}\left(\frac{I_{0}}{I}\right)=\varepsilon c l
$$

\section{Instrumentation:}

The basic components of a UV-Vis spectrophotometer are illustrated in Figure 2.1.

The UV-Vis spectrometer measures the intensity of the transmitted light of a sample compared to a reference measurement of the incident light source.

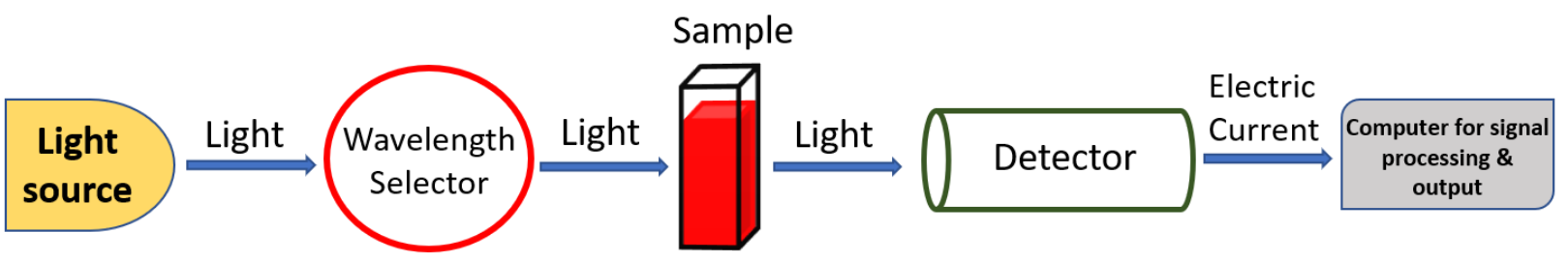

Figure 2.1 Schematic showing the main components of a UV-Vis spectrometer.

In this work, UV-Vis has been used for the characterization of AuNPs and determining concentration according to Beer's law. In this thesis, plasmon peak absorbances at 520 and 670 $\mathrm{nm}$, was used to determine the degree of aggregation. Moreover UV-Vis has also been used to characterize the AuNPs in samples containing saliva and to differentiate between aggregation and surface adsorption observed in AuNPs in a biological matrix.

Many examples of works involving AuNPs aggregation sensors using UV-Vis can be found in the literature. ${ }^{130,131,132,133}$ 


\subsection{Circular dichroism spectroscopy (CD)}

$\mathrm{CD}$ is a type of absorption spectroscopy which uses circularly polarized light and is only applicable for optically active i.e. chiral molecules. It is based on the differential absorption of left and right circularly polarized by a chiral molecule which absorbs the two polarizations with different efficiency. This difference in the absorption is measured to gain information on the structure of the molecule. ${ }^{134}$ In this work CD has been used to investigate conformational changes in DNA aptamers upon target binding.

\section{Instrumentation}

Light is linearly polarized and passed through a monochromator. The single wavelength light is then passed through a photo elastic modulator which circularly polarizes it such that the incident light on the sample switches between right and left circularly polarized light. As the incident light switches direction of polarization, the absorption changes and the differential molar absorptivity can be calculated. The most valuable application of CD in case of biomolecules comes with the possibility to monitor conformational changes in molecules. The schematic showing the working of a CD spectrometer has been shown in Figure 2.2.

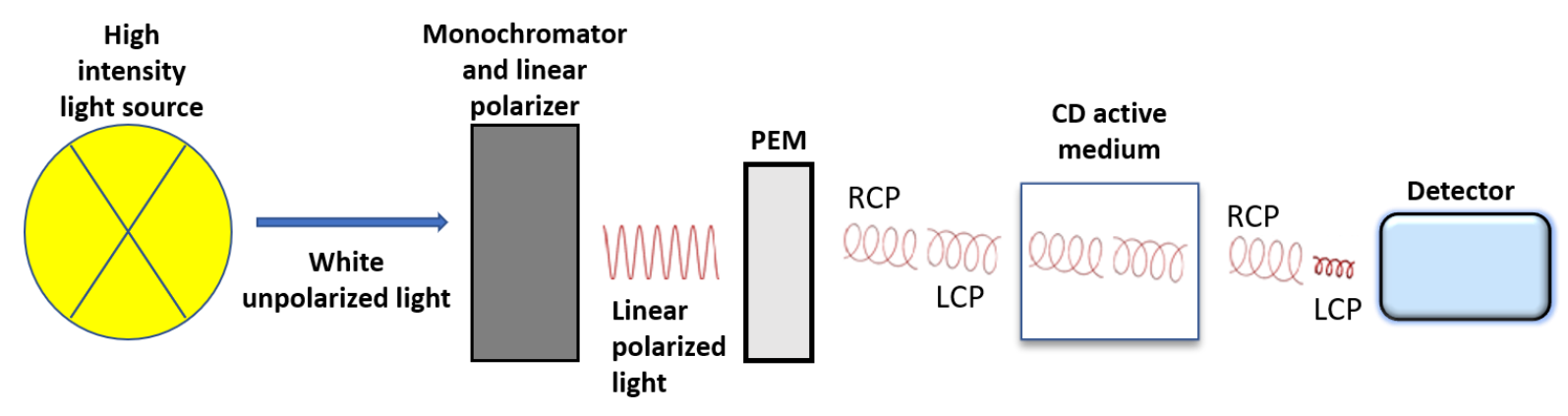

Figure 2.2 Schematic showing the working of a CD spectrophotometer

An optically active molecule will absorb two directions of circularly polarized light by different amounts, given by:

$$
\Delta A=A_{l}-A_{r}
$$


Where $\Delta A$ is the difference in absorbance and $A_{l}$ and $A_{r}$ signify the left absorption and right absorption of the circularly polarized light. Then, the Lambert-Beers law can be re-written as:

$$
A=\left(\varepsilon_{l}-\varepsilon_{r}\right) c l
$$

Where $\Delta \varepsilon=\left(\varepsilon_{l}-\varepsilon_{r}\right)$, is the molar circular dichroism representing the difference in molar absorptivity.

The absorption of the incident light by a chiral molecule changes the amplitude and the intensity and the square of the amplitude of the incident light. Since the molar absorptivity of LCP and RCP are different, therefore the amplitudes of the RCP (right circularly polarized) and LCP (left circularly polarized) light are also different and the resulting light wave is elliptically polarized.

The historically reported unit of $\mathrm{CD}$ experiments is molar ellipticity [ $\theta]$, which removes dependence on concentration and path length.

$$
\begin{aligned}
& {[\theta]=32.98 \Delta A} \\
& {[\theta]=3298 \Delta \varepsilon}
\end{aligned}
$$

Where 3298 converts from the units of molar absorptivity to degrees. $\mathrm{cm}^{2} . \mathrm{dmol}^{-1}$

In DNA molecules, the chirality exists because of the asymmetric backbone sugars and the helical arrangement of its constituents. Natural DNA most commonly adopts the B-form representing right-handed helix, with characteristic peaks at $280 \mathrm{~nm}$ (positive) and $245 \mathrm{~nm}$ (negative). The specific CD spectrum however is distinctive for each DNA depending on its sequence composition. RNA molecules most commonly adopt the A-form duplex which is characterized by a positive peak at $260 \mathrm{~nm}$ and a negative peak at $210 \mathrm{~nm}$. B-DNA form can undergo a shift to the Z-form (left-handed helix) by increasing the ionic strength. This was first observed by Pohl and Jovin. ${ }^{135}$ The B-Z transition can be induced by both salt and alcohol. ${ }^{136,137}$ It was found that RNA was also able to switch to the left hand structure may be due to functional reasons. ${ }^{138,139}$ Other structures such as the distinctive G-quadruplex structure can also be adopted by an initially B-form DNA with Guanin-rich regions. This transition may be brought about by an analyte such that the DNA undergoes a distinctive conformational change to a $\mathrm{G}$ quadruplex to accommodate the analyte in it's binding domain. ${ }^{140,141,142}$ 


\subsection{Dynamic light scattering (DLS) and Zeta-potential (ZP) characterization of nanoparticles}

In this work, DLS and ZP have been strictly used to only characterize and probe nanoparticle size and stability changes under different conditions. Therefore, the discussion on these techniques will be limited according to the scope of this work.

\subsubsection{DLS}

DLS has been used to characterize the hydrodynamic size of citrate stabilized AuNPs, AuNPs coupled with aptamers and AuNPs in a biological sample to characterize the surface adhesion between biomolecules and AuNPs which results in an increase in size.

\section{Instrumentation and principle}

Malvern Zetasizer nanoDLS has been used in this study. It uses a $4 \mathrm{~mW}$ He-Ne laser of $638 \mathrm{~nm}$. The APD (avalanche photodiode) detector is placed at $90^{\circ}$ which is less efficient in detecting backscattering than when placed at $173^{\circ}$ in other modern versions. Optically translucent plastic disposable cuvettes have been used with this instrument which require at least 1- $2 \mathrm{~mL}$ of sample.

The principle of DLS also known as photon correlation spectroscopy or quasi-elastic light scattering is based on measuring the scattered light intensity of the incident laser by a spherical particle in colloidal dispersion. The working principle can be seen in Figure 2.3. DLS accounts for the random Brownian motion of the spherical particles which scatter the incident light. The scattered light is collected by detector placed at $90^{\circ}$ for forward scattering or $173^{\circ}$ for backscattering and the fluctuations in the intensity of the scattered light correlated against decay times intervals such that the intensity autocorrelation function is obtained which is used to calculate the translational diffusion coefficient. ${ }^{143}$ 


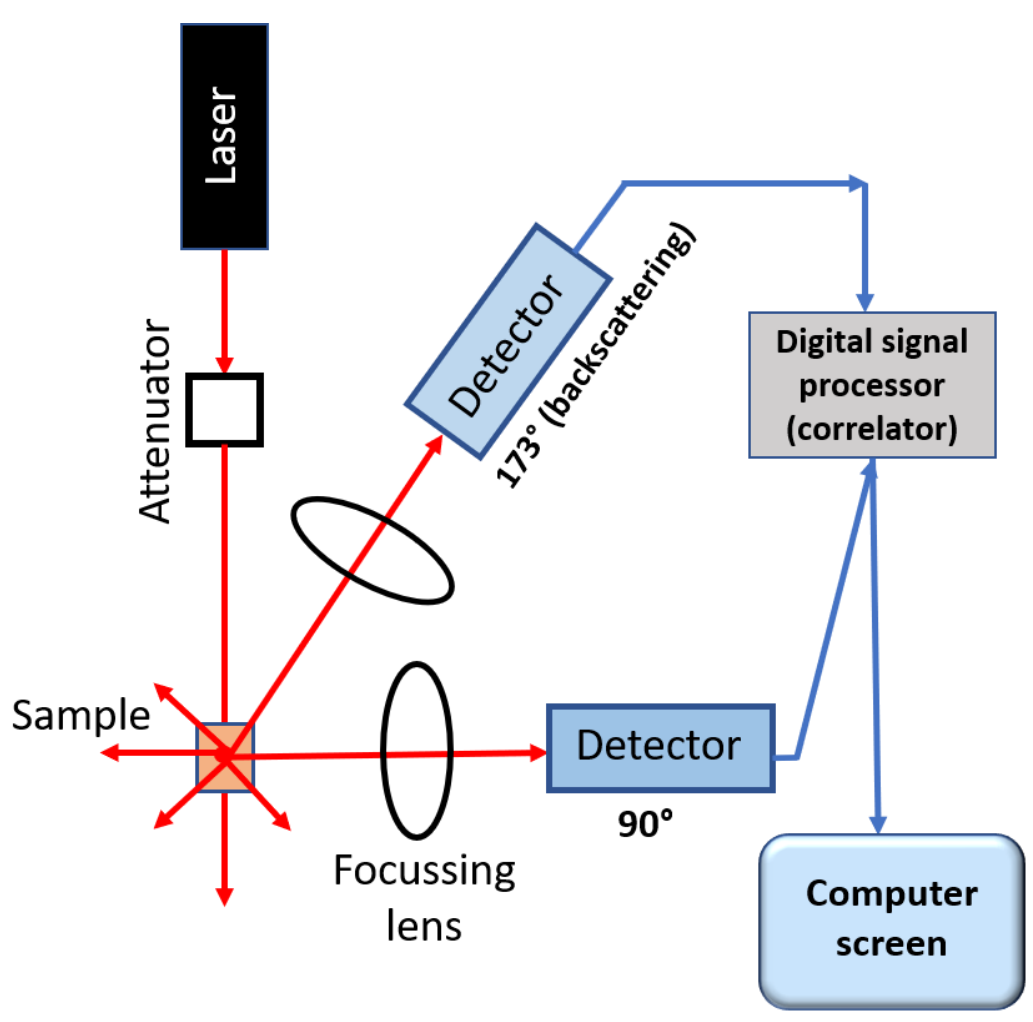

Figure 2.3 Working principle of a DLS instrument

The translational diffusion coefficient of the particles is measured, and the hydrodynamic size (nm) is calculated using the Stokes-Einstein's equation

$$
D_{t}=\frac{k_{B} T}{6 \pi \eta R_{H}}
$$

or

$$
D_{H}=\frac{k_{B} T}{3 \pi \eta D_{t}}
$$

Where, $D_{t}=$ translational diffusion coefficient, $k_{B}=$ Boltzmann constant $\left(1.38 \times 10^{-23} \mathrm{~J} / \mathrm{K}\right), \mathrm{T}=$ temperature, $\eta=$ absolute viscosity, $R_{H}=$ hydrodynamic radius and $D_{H}=$ hydrodynamic diameter It is evident from the equation, the hydrodynamic size or the DLS results depends on a lot of factors, such as viscosity of the solvent, instrument temperature, and refractive index of the 
solvent. The hydrodynamic size measured by DLS is the actual core size of the particle wrapped within a layer of hydrated/solvated outer layer whose composition changes with time.

Wang et al. ${ }^{144}$ has demonstrated the use of DLS as a facile and powerful tool for systemic investigation of surface interactions between AuNPs and DNA by monitoring the changes in the particle size of AuNPs. DLS was used to evaluate the kinetic adsorption profile and the changes in the conformation of both thiolated and non-thiolated DNA on the surface of AuNPs. DLS has also been used to probe surface interactions between biomolecules such as proteins and dispersed NPs. Performing DLS in biological matrices can be quite challenging owing to the dynamic nature of the adsorbed layers on the NPs and the constant fluctuations of the $R_{H}$ over time. ${ }^{143}$

\subsubsection{Zeta potential}

In this work, zeta potential has been used in order to evaluate the stability of citrate stabilized AuNPs solutions and confirmation of the decrease in the stability or the surface charge upon removal of excess citrate from AuNPs solution by washing. Moreover, zeta potential has also been used to confirm the adsorption of aptamers (highly negatively charged DNA oligonucleotides) which impart further stability upon adsorption to AuNPs, indicated by a more negative zeta potential value.

\section{Instrumentation and principle}

Colloidal particles in solution have a surface charge which enables them to stay suspended in a colloid. The net charge on the particle surface results in the development of surrounding charges that are opposite in charge and exist close to the surface. This gives rise to an electric double layer existing around each particle. The liquid layer around the article can be divided into two parts: an inner stern layer consisting of tightly bound counter ions to the surface of a charged particle, and a diffuse layer consisting of both counter ions and ions with the same charge as the surface loosely associated with the particle. When the particle moves due to gravity or under an applied electric field, the particles within the diffuse layer move it and particles beyond the boundary of diffuse layer carry the bulk dispersant charge such that a potential exists at the boundary of the diffuse layer or the slipping pane. This potential at the boundary of the slipping pane is known as the zetapotential. Zeta potential (also termed as electrokinetic potential), measures the potential difference existent between the slipping pane and the stern layer or the electric double layer of a 
colloidal particle moving under an applied electric field. Figure 2.4 highlights the principle of zeta potential measurement. ${ }^{143}$

The magnitude of the zeta potential indicates the strength of the stability of a colloid.

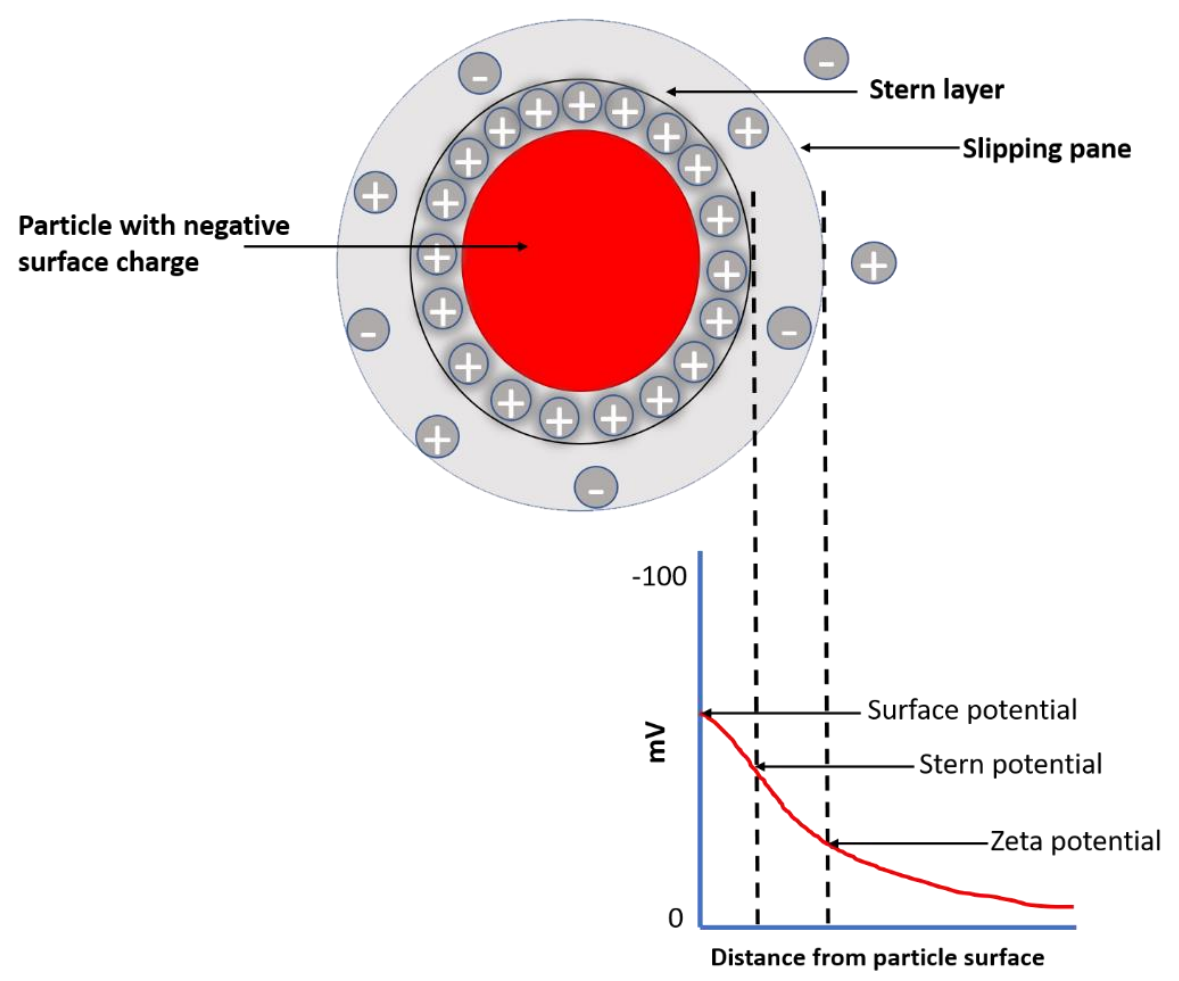

Figure 2.4 Schematic representation of the theory of zeta potential

For zeta potential measurements an incident light source from a laser is split into two to give rise to a reference beam and a incident beam which passes through the middle of the sample illuminating it and the scattered beam is then detected at an angle of 13 by a detector. Under an electric field, any particle moving through the detection volume will cause the intensity of the light detected to fluctuate with a frequency proportional to the particle mobility or the electrophoretic mobility. This information is passed on to the detector and subsequently to a computer where the software produces the digital information the form of frequency spectrum from which the electrophoretic mobility and hence the zeta potential can be calculated. 
Zeta potential has been used to probe the surface densities of various probe molecules (eg. DNA aptamers) differing in length as well as target binding and non-target binding aptamers to probe the difference in surface density of AuNPs-aptamer systems in the presence and absence of targets. ${ }^{145,146}$ More generally, several reports of zeta potential being used in conjunction with DLS to characterize the synthesized nanoparticles can be found in the literature. Zeta potential is mainly used to study surface charge, and hence the stability of nanoparticles. ${ }^{147,148}$

\subsection{Isothermal titration calorimetry (ITC)}

In this work, the ITC has been used as qualitative technique to demonstrate binding between the DNA aptamer and analyte. Several works have demonstrated the use of ITC to investigate the nature of binding interaction between protein and ligand as well as binding thermodynamics between DNA aptamers and protein or small molecules. ${ }^{149}$ ITC has primarily been used in the field of drug designing. ${ }^{150}$

\section{Principle and instrumentation}

Isothermal Titration Calorimetry or ITC is a type of calorimetry where the heat absorbed or generated when molecules interact is measured by the instrument. The degree of heat generated or absorbed may be small or large and can be measured by these sensitive instruments. Several parameters can be obtained from an ITC experiment such as the enthalpy $(\Delta H)$, dissociation constant $\left(\mathrm{K}_{\mathrm{d}}\right)$ and stoichiometry $(\mathrm{n})$. Moreover, ITC can be used as a qualitative measurement technique to confirm a binding event and may serve as a complementary validation for assays. The thermodynamic insight gained from an ITC experiment helps to shed light on the nature of interaction between the probe and analyte, whether polar or non-polar. ${ }^{151}$ 


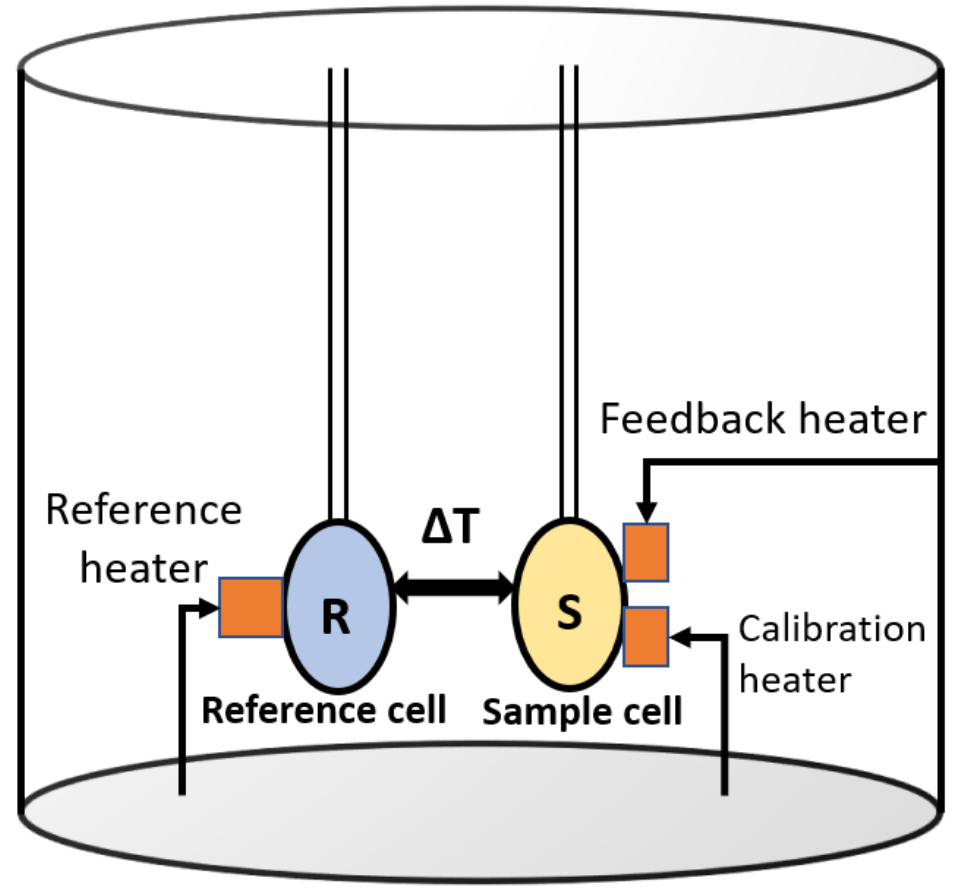

Figure 2.5 Schematic showing the principle of an ITC instrument

In a typical experiment, sample cell consists of a buffered solution of biomolecules and an analyte diluted in a matching buffer is titrated into the cell through a syringe (Figure 2.5). The heat change that occurs due to the interaction results in a difference in temperature between the reference cell and sample cell which the instrument detects and compensates to maintain the reference and sample cells at the same temperature. The power needed to maintain the isothermal condition is expressed as peaks (which represents the heats of interaction between probe and analyte) that become smaller and smaller as the probe saturates and then the constant peaks represent only heats of dilution. After the titration is complete the software integrates the individual peaks and constructs a Wiseman Plot. Thereafter, the ITC software can be used to fit the binding curve in an appropriate model to get the values of enthalpy, $\mathrm{Kd}$, stoichiometry, and the entropy, Gibbs free energy can be calculated. 


\section{CHAPTER 3}

Accounting for interaction kinetics between gold nanoparticles and aptamers enables high performance colorimetric sensors 


\subsection{Abstract}

DNA aptamers have emerged as promising probes for challenging analytes that cannot be easily detected by conventional probes, including for small molecule targets. Among the different signal transduction approaches, gold nanoparticle (AuNP) aggregation assays have been widely used to generate a colorimetric response from aptamer-target interactions. This sensor design relies on competition between the aptamer adsorbing to the AuNP surface versus interacting with the target, whereby target binding reduces the number of adsorbed aptamers which destabilizes AuNPs towards salt induced aggregation, thereby inducing a color change. However, this thermodynamic framework overlooks the potential influence of interaction kinetics of different aptamer conformations with AuNP surfaces and with targets in solution or near surfaces. Here, we show that aptamers become more strongly adsorbed on AuNPs over time, and these trapped aptamers are less responsive towards the target analyte. By varying the sequence of addition in sensing assays, we demonstrate that these interaction kinetics have a significant effect on the sensor response, and thereby produce an effective sensor for methamphetamine (meth) at biologically relevant levels in oral fluids. As well as underpinning new tools for assay development, this new knowledge also highlights the need for aptamer selection strategies that evolve aptamer sequences based on the functionality that they need to exhibit in an actual sensor. 


\subsection{Introduction}

Gold nanoparticle (AuNP)-based aggregation assays have emerged as simple, fast, cost effective yet sensitive assays with high potential for translation into on-site diagnostic systems, mostly for a yes/no or semi quantitative response. ${ }^{152,153,154}$ These assays have been demonstrated to be particularly suitable for detection of small molecules such as hormones, drugs etc. ${ }^{155,156}$ Although AuNPs do not themselves recognize targets, they are known to undergo distinct visible color changes associated with their surface plasmon resonance when changes in their surface charge induces aggregation. ${ }^{157}$ Dispersed AuNPs exhibit a red color in solution and a blue/purple color when aggregated. Bare AuNPs are unstable in solution and the rate of aggregation and flocculation are sensitive to the layer of surface charge (e.g., from citrate ions or surfactants from AuNP synthesis), ionic strength, $\mathrm{pH}$, and temperature. When biological probes such as antibodies, DNA aptamers, and peptides are adsorbed to the surface, surface charge can thus become dependent on the presence of the target, underpinning rapid and cost-effective colorimetric biosensors. ${ }^{156,158,50}$ For example, DNA aptamers - single stranded oligonucleotides also known as 'artificial antibodies' - adsorb to AuNPs via their bases. The strength of this interaction is affected by targetinduced changes in aptamer conformations such as stabilization of double stranded (hairpin) segments, typically leading to desorption from AuNPs and destabilization of AuNPs towards aggregation in the presence of the target. ${ }^{83,90,159,47}$

Previous studies have shown stronger affinity and faster adsorption kinetics towards AuNPs of single stranded (ss) DNA over double-stranded (ds) DNA ${ }^{71,160}$ owing to their higher structural flexibility and availability of bases for surface interaction. ${ }^{161}$ The binding affinity of ssDNA adsorption is largely dependent on the composition of bases as well as the oligonucleotide length. Moreover, longer DNA strands have a slow adsorption rate and a slower desorption rate which makes binding of longer ssDNA comparatively more favourable than short ssDNA, which have a smaller Langmuir constant. Longer DNA strands impart greater stability and protection to AuNPs surface against salt induced aggregation, whereas shorter DNA strands offer more loading capacity per AuNP. ${ }^{162}$

The nature and magnitude of driving forces for DNA adsorption to AuNPs has been debated. Initial attempts to explain the disparity in adsorption between ss and ds DNA ${ }^{161}$ relied on simple DLVOlike electrostatic ${ }^{163}$ interactions. However, follow up studies contradicted earlier assumptions 
about the nature of DNA-AuNP interactions, and led to the suggestion of hydrophobically driven adsorption. ${ }^{91,164}$ Here, the different solvation capabilities of ss and ds DNA, and the varying solvation properties of individual bases are invoked to explain the conformational and base dependent adsorption of ss DNA on AuNPs. Meanwhile, new versions of electrostatic models gained experimental and theoretical support, in which ss DNA can adsorb to AuNPs by displacing citrate ions, in an equilibrium that depends on the salt concentration. ${ }^{165}$ Moreover, in a low ionic strength environment, DNA is thought to adopt a more extended conformation on the AuNPs surface, and this may happen dynamically after initial adsorption has occurred. ${ }^{165}$ The involvement of specific chemical bonding interactions between individual bases and AuNPs - such as through the $\mathrm{N} 3$ and N7 sites of adenine and guanine bases ${ }^{165}$ - can also account for variation in binding affinities as a function of oligonucleotide sequence. ${ }^{93,166,167}$ These new insights and ongoing debates show that DNA adsorption to AuNPs is a non-trivial phenomena, and likely reflects a balance of electrostatic, hydrophobic and specific covalent interactions that are strongly dependent on $\mathrm{pH}$, ionic strength, conformation, length, composition, and sequence/structure of oligonucleotides. ${ }^{92}$

While our understanding of binding thermodynamics remains incomplete, less attention has been given to details of structure based adsorption kinetics. Since the binding affinity depends on the DNA structure adopted amongst a vast parameter space of surface-binding modes and folding conformations, binding and rearrangement kinetics must also be a factor. ${ }^{92,162,168}$ Thus, we hypothesize that the nature and strength of aptamer-AuNP interactions could change significantly over time, with a strong likelihood of extensively restructured aptamer conformations becoming trapped on AuNP surfaces and unresponsive towards the target. It follows that the competition between aptamer-AuNP and aptamer-target interactions would also be time dependent, which would suggest different strategies for creating a strong sensor response. Put differently, we question whether AuNP aggregation assays are even conducted at equilibrium - with facile exchange between aptamers adsorbed to AuNPs and free in solution, or whether the sensor response actually relies on metastable aptamers whose prevalence diminishes over time. For small molecule targets, the challenge may be compounded because such aptamers typically have small ligand binding domains and modest structural changes driven by the target binding, leaving them more vulnerable to being dominated by interactions with AuNPs. 
In this work the stability of aptamer-coated AuNPs was examined and it was found that over time, they become significantly more resistant to salt-induced aggregation, and their sensor response suppressed accordingly. This finding prompted to rethink about the sequence of steps in the sensor assay. Instead of conducting a dissociation assay with aptamer coated AuNPs, the sequence of addition of AuNPs was inverted in an association assay as shown in scheme 3.1. Here, the free aptamer is first incubated with the target, followed by AuNPs. Target-bound aptamers are less likely to adsorb to AuNPs, and salt-induced aggregation can be triggered before aptamers become trapped on surfaces. It was shown that the inverted association assay significantly enhances the sensitivity compared with the dissociation assay. The limit of detection (33.3 nM) surpasses previously published work and is a direct result of our new understanding about AuNP-aptamer interaction dynamics. Finally, by establishing the importance of aptamer-AuNP interaction kinetics on the sensor performance, this work highlights the need for new SELEX strategies that select aptamers on the basis of how they will function in sensor devices. 
Scheme 3.1 Possible mechanism of the signal enhancement for the aptamer dissociation assay (target last) format compared to the aptamer association assay (target first) format.

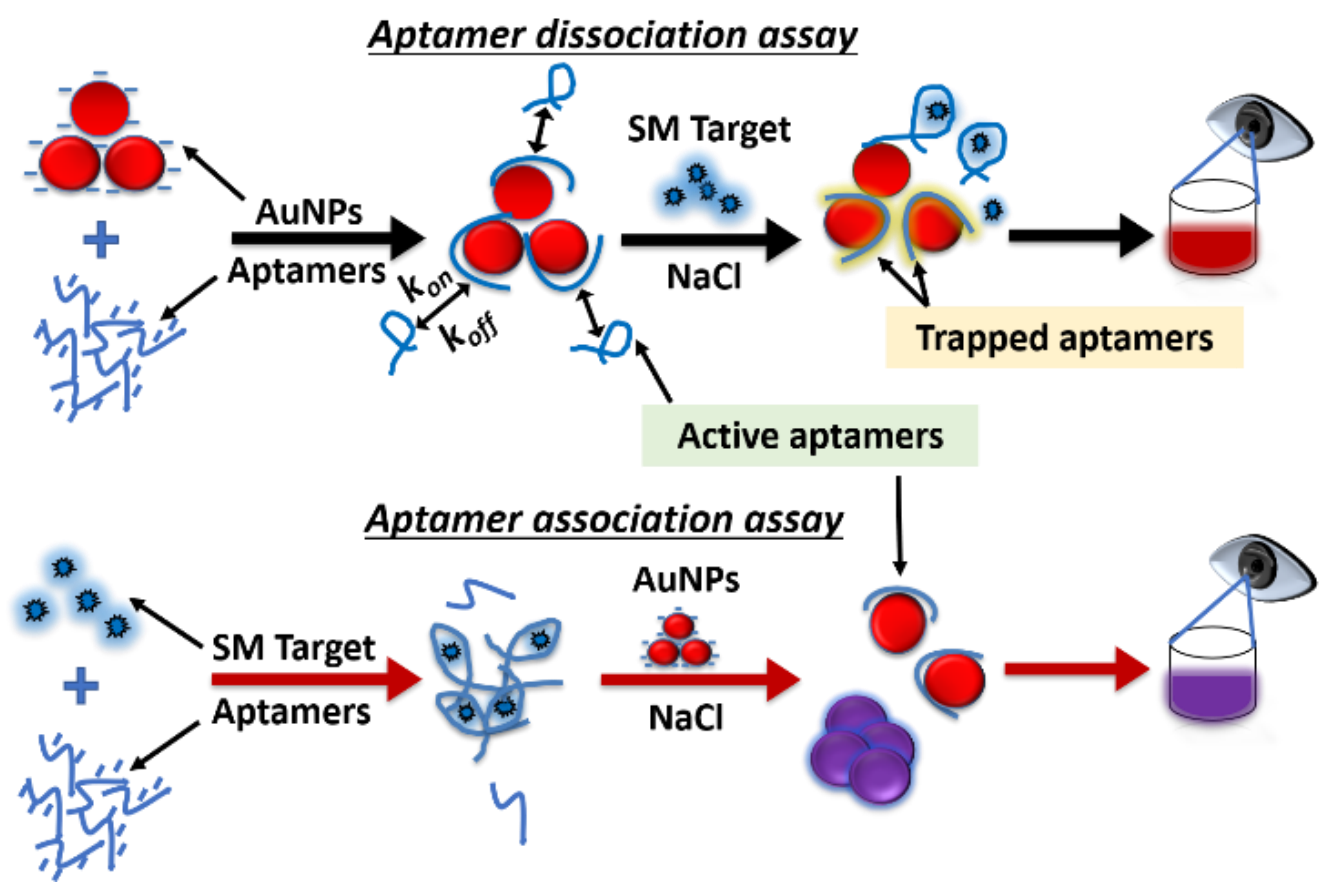

\subsection{Experimental section}

\subsubsection{Reagents and chemicals}

Methamphetamine-HCl (meth/MA), MDMA-HCl, MDA-HCl, amphetamine-HCl (Amp), ephedrine- $\mathrm{HCl}$ (Eph), pseudoephedrine- $\mathrm{HCl}$ (Pseudo) was provided by our collaborators, Institute of Environmental Science and Research (ESR) NZ. Chloroauric acid $\left(\mathrm{HAuCl}_{4}\right)$ and sodium citrate were purchased from Sigma-Aldrich. Binding and washing buffer (BWB) were prepared as follows (2 mM Tris- $\mathrm{HCl}$ at $\mathrm{pH} 7.5,10 \mathrm{mM} \mathrm{NaCl}, 0.5 \mathrm{mM} \mathrm{KCl}, 0.2 \mathrm{mM} \mathrm{MgCl} 2,0.1 \mathrm{mM} \mathrm{CaCl}$ ). All chemicals for BWB purchased from Sigma Aldrich. Deionized water (Milli-Q), class resistivity $18 \mathrm{M} \Omega$-cm was used in all experiments (unless stated otherwise), and all other chemicals were of analytical grade. Meth aptamer ABL Met S-03 75-mer aka parent aptamer (or untruncated aptamer), truncated Met S-03 34-mer, random 34-mer DNA were purchased from Alpha DNA. The parent aptamer 75-mer was produced by SELEX technique which was conducted via similar methods reported for the generation of an E2 binding aptamer. ${ }^{67}$ An affinity-based SELEX 
approach was used in which the meth target was tethered to agarose beads. The selection buffer used for SELEX had the same composition as BWB except for the presence of $0.05 \%$ Pluronic. The ssDNA preparations were dissolved in Tris-EDTA (TE) buffer (10 mM Tris-HCl, $1 \mathrm{mM}$ disodium EDTA, $\mathrm{pH} 7.5$ ) and kept at $-5^{\circ} \mathrm{C}$ before use.

Table 3.1: Sequences of the used oligonucleotides (in 5'-3' direction)

\begin{tabular}{|l|l|}
\hline Type & Sequence 5'-3' \\
\hline $\begin{array}{l}\text { ABL Met } \\
\text { S-03 ATACGAGCTTGTTCAATAGCGTTTCTATCTGGCTGTATCGTGATAGT } \\
\text { (Parent 75- } \\
\text { mer) }\end{array}$ & AAGAGCACTAATGATAGTAAGAGCAATC \\
\hline $\begin{array}{l}\text { Truncated } \\
\text { Met S-03 } \\
\text { 34-mer }\end{array}$ & \\
\hline $\begin{array}{l}\text { Random } \\
\text { 34-mer }\end{array}$ & AGGGCTTAGATCTTAGCTATATCGTGAGCACTAA \\
\hline
\end{tabular}

\subsubsection{Methods}

\section{Synthesis and characterization of citrate stabilized AuNPs}

AuNPs were synthesized by the reduction of $\mathrm{HAuCl}_{4}$ by tri-sodium citrate in solution. $1 \mathrm{mM}$ of $\mathrm{HAuCl}_{4}$ solution is brought to a boil followed by the addition of sodium citrate. Within one minute the pale-yellow color of solution fades to a colorless before turning into a bright red, signaling the formation of AuNPs. The concentration of AuNPs was estimated via UV-Visible spectroscopy to be approximately around $13 \mathrm{nM}$ from calculation based on the Beer Lambert law using an extinction coefficient of $2.01 \times 10^{8} \mathrm{M}^{-1} \mathrm{~cm}^{-1}$ at $520 \mathrm{~nm}$. ${ }^{101}$ The aggregation maxima was located at $670 \mathrm{~nm}$. The surface potential of purified citrate stabilized AuNPs (11 nM) was found to be -25.6 $\pm 0.4 \mathrm{mV}$ (reported as average value of triplicates \pm Standard deviation) from zeta potential measurements and the average size measurements by DLS suggested an approximate diameter between $15-20 \mathrm{~nm}$. 


\section{AuNPs-Aptamer incubation for aptamer dissociation assay [(AuNPs,Aptamer) +Target]}

$1 \mathrm{~mL}$ of AuNPs was mixed with $3.3 \mu \mathrm{L}$ from a $10 \mu \mathrm{M}$ stock of 75 -mer and/or 34-mer aptamers in an optimized stoichiometric ratio 3:1 Aptamer: AuNPs, vortexed for 1 minute and incubated for no less than 30 minutes in an Eppendorf tube. The mixture was then transferred to a 96-microtitre plate and exposed to $12 \mu \mathrm{L}$ of buffer or target solution. Incubation for less than 30 minutes was found to be insufficient for successful adsorption.

\section{Target-Aptamer incubation for aptamer association assay [(Target,Aptamer) + AuNPs]}

Briefly, $0.33 \mu \mathrm{L}$ from a $10 \mu \mathrm{M}$ Aptamer working stock were mixed with $12 \mu \mathrm{L}$ of buffer or target solution and incubated for 15 minutes in an Eppendorf tube before addition of $100 \mu \mathrm{L}$ of AuNPs. The (34-mer, meth) + AuNPs mixture was left for no less than 30 minutes and an optimized concentration of salt added, followed by a visual color change which was quantified by UV-Vis spectroscopy.

\section{Salt titration experiments for finding the optimal ionic strength}

The optimal salt concentration was determined by titrating various volumes of $0.5 \mathrm{M} \mathrm{NaCl}$ into bare AuNps, bare AuNPs + $100 \mathrm{nM}$ meth, (AuNPs,75-mer) and (AuNPs,75-mer) + $100 \mathrm{nM}$ meth for aptamer dissociation assay format. The optimization procedure was repeated for truncated and random DNA sequences. The same steps with changing the sequence of addition of AuNPs (added last in case of target samples) were repeated for aptamer association assay format. The corresponding ratio of absorbances at $670 \mathrm{~nm}$ and $521 \mathrm{~nm}$ were recorded as a measure of degree of aggregation.

\section{Colorimetric sensing assay and data collection}

In a typical sensing assay $100 \mu \mathrm{L}$ of (AuNPs + Aptamer) probe was mixed with $12 \mu \mathrm{L}$ of buffer (for reference samples) or target and incubated for 15 minutes before adding $8 \mu \mathrm{L} \mathrm{NaCl}$ to give a total reaction volume of $120 \mu \mathrm{L}$. After 10 minutes of reaction, UV-Vis data was collected using an Envision multilabel plate reader at $521 \mathrm{~nm}$ and $670 \mathrm{~nm}$ and the ratio of absorbances at 670/521 
was simultaneously calculated by the software. The ratio $\mathrm{A}_{670} / \mathrm{A}_{521}$ was used to evaluate the response of the sensor throughout the experiment. The absorption maxima varied between 520$521 \mathrm{~nm}$ for different AuNPs batches after aptamer adsorption, thus UV-Vis characterization was done every time after a new AuNPs batch was prepared and prior to plate reading analysis.

\section{SYBR green assay $I$}

Parent 75-mer aptamer was mixed in BWB solution with the fluorescence dye SG then a Meth titration was conducted, a fluorescence signal is monitored along the Meth titration. $10 \mu 1$ of SG (7x) and $10 \mu \mathrm{l}$ of aptamer $(10 \mu \mathrm{M})$ were mixed. A range of Meth concentrations varying from 0 to $1 \mu \mathrm{M}$ was prepared in BWB and added directly to the SG-aptamer solution to deliver a final volume of $1000 \mu \mathrm{L}$. The fluorescence emission spectra were recorded from 500 to $650 \mathrm{~nm}$ using an excitation wavelength of $497 \mathrm{~nm}$. The emission fluorescence at $520 \mathrm{~nm}$ was measured.

\section{Time dependent salt titration experiments to monitor optimal salt concentration}

In order to probe any changes in the optimal salt concentration over time, salt titration experiments as described previously were performed to find out the optimal salt concentration after (AuNPs, Aptamer) incubation for 30, 60 and 90 minutes. The relative absorption at $521 \mathrm{~nm}\left(\mathrm{~A}_{521}\right.$ (rel.)) was plotted vs ionic strength to evaluate the degree of aggregation.

\section{Zeta-potential measurements}

To monitor the stability of interactions between aptamer and ANPs over time, zeta potential measurements were performed on a Zetasizer Nano ZS equipped with a $633 \mathrm{~nm}$ laser (Malvern instrument, UK). Samples were prepared as described previously. ${ }^{67}$

\section{Selectivity of the aptasensors}

In order to investigate the specificity of the aptasensor, the truncated 34-mer aptamer sequence was mixed with meth or other potential interferants (from the amphetamine class of drugs) including meth pre-cursor such as amphetamine, ephedrine, pseudoephedrine, MDA and MDMA, each at a concentration $200 \mathrm{nM}$. 


\section{Circular dichroism studies}

In a typical experiment, $1.5 \mu \mathrm{M}$ of 34-mer aptamer in BWB (without target) and with $3 \mu \mathrm{M}$ of target in BWB was analysed in a CD instrument. $\mathrm{CD}$ analysis was performed in a $1 \mathrm{~mm}$ path length Quartz cuvette in a Chirascan spectrometer. The spectra were recorded from 380 to $180 \mathrm{~nm}$ at $20^{\circ} \mathrm{C}$ and corrected by subtraction of background scan of BWB.

\section{Isothermal titration calorimetry}

Calorimetric experiments were achieved for the aptamer and a mutated version. Isothermal titration calorimetry (ITC) assays were performed on an Affinity ITC system (TA Instruments Inc., New Castle, DE, USA). $100 \mu \mathrm{M}$ of 34-mer aptamer was injected into the sample cell and $1 \mu \mathrm{L}$ of $5 \mathrm{mM}$ meth was programmed to be added into the cell at a regular interval of 3 minutes for 30 injections after loading into the syringe. The experiment was carried out at $20^{\circ} \mathrm{C}$ under $125 \mathrm{rpm}$ stirring. The samples were degassed for 30 minutes before use. The reference cell was maintained at the same volume as the sample cell.

\subsection{Results and discussion}

Investigations began by considering the sensing responses of the 75-mer meth binding aptamer in BWB buffer following the conventional aptamer dissociation assay. The 75-mer aptamer was chosen among the four, meth binding parent aptamer candidates after a screening using the colorimetric aptasensing assay which revealed the 75-mer as the most responsive aptamer to meth (Figure A1). Successful adsorption of aptamers on AuNPs was confirmed via salt titration which revealed increasing resistance towards aggregation with increasing aptamer coverage (Figure A3 A) and DLS measurements (Figure A2 B). The optimal ionic strength was found to be $50.6 \mathrm{mM}$ (Figure A5 A). This is the ionic strength at which the bare AuNPs are fully aggregated and the (AuNPs, Aptamer) are on the verge of instability therefore most sensitive to the presence of target which can induce a conformational change leading to desorption from and considerable aggregation of AuNPs causing a visible color change. Relative sensitivity investigations showed that the most sensitive response was obtained at an [Aptamer]:[AuNPs] molar ratio 3:1. (Figure A3 B). 


\section{Colorimetric sensing using dissociation assay}

Sensing response of the parent 75-mer meth binding aptamer can be seen in Figure 3.1, (AuNPs, Aptamer) samples were mixed with various concentrations of meth $(0,5,10,20,50,100,200$, 500,1000 and $1500 \mathrm{nM}$ ). This range was chosen because of our aim to reliably detecting meth at the legal threshold for oral fluids of $\sim 167 \mathrm{nM}$ or $25 \mathrm{ng} / \mathrm{mL}$. As seen in Figure 3.1, the lowest level of meth that can be detected visually is $1 \mu \mathrm{M}$ with a linear detection range of $0.1-1 \mu \mathrm{M}$ and an $\mathrm{R}^{2}$ $=0.94$ (refer to Figure A6 A for the calibration curve). No detection signal was observed (neither with naked eye nor UV-Vis) below $0.1 \mu \mathrm{M}$ meth. The calculated Level of Detection (LOD) using $3.3 \times$ (Standard error of intercept $\div$ Slope) was found to be $260 \mathrm{nM}$. The colorimetric LOD achieved is higher than the cut-off levels of detection at which meth is to be monitored in real scenarios. It was confirmed that the sensor response was triggered by the aptamer coating by observing no response with bare AuNPs exposed to meth indicating meth triggered aggregation of AuNPs is negligible at the concentrations tested (Figure 3.1). Only at concentrations above 20 $\mu \mathrm{M}$, meth triggered aggregation of AuNPs gradually leading to total aggregation at concentrations $\sim 0.5 \mathrm{mM}$ meth. Also, after addition of salt, the bare AuNPs-meth samples all destabilize completely due to the absence of protection by aptamers (Figure 3.1). This indicated that meth had no stabilizing/protection effect on the AuNPs.

Having ruled out two potential effect of SMs interference with the assay, it was also important to evaluate the effect of meth on aptamer adsorption i.e. whether meth inhibited aptamer adsorption on the AuNPs as has been observed with other SMs. ${ }^{113}$ The results obtained (Figure A7) showed no such effect at $500 \mathrm{nM}$ meth rather, at a higher meth concentration of $50 \mu \mathrm{M}$ meth it was observed that the meth did interact with AuNPs (which is expected owing to the primary amine group and a net positive charge on meth at physiological $\mathrm{pH}$ ) and destabilized them on its own, therefore it was concluded that meth concentration above $20 \mu \mathrm{M}$ were unsuitable for application to colorimetric assays. Lastly a negative control aptamer was tested to validate the observed response. A 17ß-Estradiol binding aptamer (35-mer E2) was used for that purpose and the lack of a binding response successfully confirm the that the observed response with the meth binding aptamer was because of the meth induced desorption of the aptamer (Figure 3.1) ${ }^{67}$ Apart from the control studies to rule out non-specific response, an independent technique to evaluate the affinity or the dissociation constant (Kd) between the aptamer and meth was investigated by 
Fluorescence Syber green assay (Figure A9). The Kd obtained was $200 \mathrm{nM}$ which further demonstrated the credibility of the response obtained via the colorimetric assay.

The sensitivity of the assay was enhanced then by truncating the parent 75-mer sequence into a 34-mer sequence (see Figure 3.1). The truncations were done systematically to ensure no loss of binding activity. Various different truncated aptamer sequence with varying truncated lengths were tested before establishing a 34-mer truncated sequence being most promising. In the past, several reports showed enhanced detection sensitivity by using truncated or shortened aptamer versions of the parent sequence, because flanking regions that are not directly involved in target binding can impede aptamer dissociation from the AuNPs, regardless of whether the target is bound to the aptamer. $^{169,67}$

Truncated aptamer structures were predicted using Mfold. ${ }^{170}$ The 75-mer aptamer was shortened to generate a 34-mer meth binding aptamer, estimating the binding pocket to be around the double stranded stem loop region indicated in Figure 3.2.

Figure 3.1 shows the response of the aptasensors occurring at different concentrations of meth. The optimal salt concentration for the truncated 34-mer aptamer was found to be $30 \mathrm{mM}$ consistent with the idea that longer aptamers provide better protection against salt once adsorbed compared to shorter aptamers. ${ }^{165}$ As seen in Figure 3.1, there is a slight visible improvement in the detection sensitivity with the truncated 34-mer which is evident if compared with the naked eye detectable color change occurring for the two sequences. Ideally, visible color changes at the cut off level of meth $(\sim 167 \mathrm{nM})$ for practical applications are highly desirable due to potentially eliminating the need for an instrument and assist in an instant yes/no type of output. However, as demonstrated this clearly could not be achieved with the truncated 34-mer using the dissociation assay.

The LOD achieved spectrophotometrically was $50 \mathrm{nM}$ with a linear detection range of 50-500 $\mathrm{nM}$ and $\mathrm{R}^{2}$ value 0.97 (refer to Figure A6 $\mathbf{B}$ for the calibration curve). The calculated LOD using the standard formula $3.3 \mathrm{x}$ (standard error of intercept $\div$ Slope) was $81.2 \mathrm{nM}$. A 3-fold improvement in LOD was observed compared to the parent 75-mer.

The observed 3-fold improvement in LOD from the truncation approach is much smaller than those reported previously, which report up to 25 -fold improvement from truncations. ${ }^{67}$ To account for the lack of improvement in sensitivity upon truncation, it was suggested that aptamers may become 
stuck on AuNPs in conformations that do not respond to the presence of the target. Indeed, it has been previously demonstrated via NMR spectroscopy that in a cocaine-binding aptamer, doublestranded DNA interactions are essential for target binding, yet these will be disrupted via interaction with AuNPs. ${ }^{171}$ It has also been reported previously that strong binding interactions between AuNPs and aptamers may lead to decreased sensitivity or total sensor failure. ${ }^{172,173,174}$ Therefore, it was imperative to gain more knowledge on the nature of the adsorption interactions between aptamers and AuNPs.

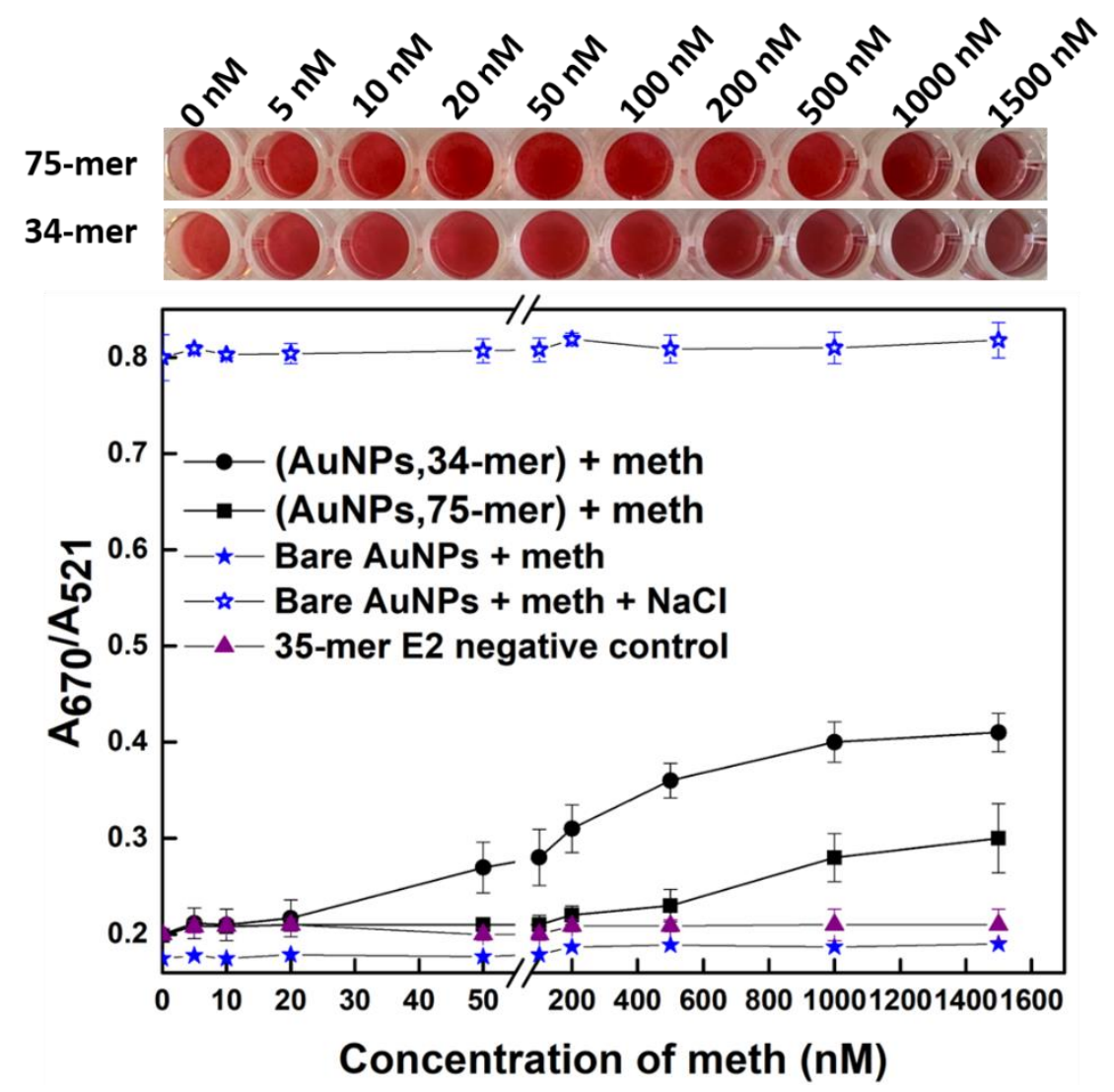

Figure 3.1 Colorimetric response of the parent 75-mer and truncated 34-mer towards a range of meth concentrations using the aptamer dissociation assay format. Error bars represent standard deviation of the mean of three measurements. 


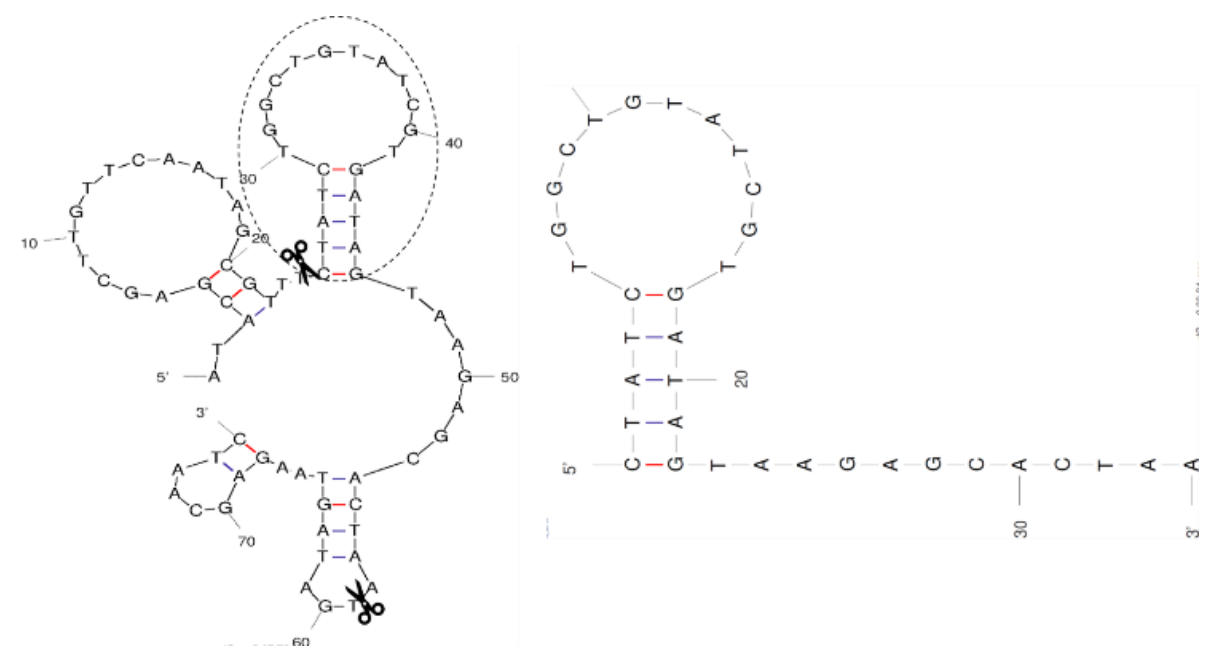

Figure 3.2 Secondary structure of the 75-mer aptamer predicted by the Mfold program indicating the truncation positions represented by a pair of scissors. The generated 34-mer truncated aptamer consists of the probable binding region (stem loop domain).

\section{Investigation of tight binding interactions between AuNPs and aptamer}

To further understand the nature of aptamer-AuNP interactions, it was investigated whether there was a time-dependent drift in the adsorption strength of aptamers to AuNPs. This was done by measuring the salt concentration required to elicit the highest response at a given concentration of target/Aptamer/AuNPs. It was postulated that in a closed system consisting of an optimized ratio of [aptamers] : [AuNPs], the optimized salt concentration required to elicit the strongest response or destabilize the AuNPs colloid would remain unchanged with time. On the other hand, if the metastable aptamers fall into a thermodynamic stable state (by being more strongly bound to the AuNPs) in a time dependent manner, this will result in a higher average surface coverage of aptamers, strong surface binding, and ultimately a higher salt concentration required to trigger a colorimetric response. It was observed that with time the optimal salt concentration shifted to higher concentrations, which indicated that the sensor became less sensitive to meth over time indeed suggesting that aptamers become thermodynamically stabilized on AuNPs and less functionally available over time (Figure 3.3 A,B). A shift of the salt concentration needed to trigger aggregation clearly suggested an increased aptamer protection which is only possible when 
target binding aptamer structure rearranges/disintegrates to ssDNA structures and provides the observed protection.

To check that the time-varying resistance to salt is caused by a stabilizing interaction of adsorbed aptamers with AuNPs over time, the time-dependence of the zeta potential was measured. From Figure 3.4 it was seen that the negative zeta potential value for the AuNPs + 34-mer system increased in magnitude over time, and the difference between the zeta- potential values with and without meth commensurately diminished over time - consistent with the suppressed sensor response over time.

To understand more about how the stability increased over time, the zeta potential dynamics of the AuNP-34mer was compared with a system comprising of a randomized version (Figure A11) of the same aptamer sequence. Although both sequences featured the same total base composition, the only difference was that the target-binding 34-mer aptamer featured a longer double-stranded stem region, which was expected to change the way that it interacted with AuNPs. It is known that sequences with longer double-stranded stem regions (typically from target binding aptamers) adsorb to AuNPs at a lower density compared with sequences with shorter double stranded regions that have more conformational flexibility. ${ }^{145}$ While the negative magnitude of the zeta potential for the 34-mer aptamer system increases from $-33 \mathrm{mV}$ to $-43 \mathrm{mV}$ from 30 - to 90 -min incubation, the random aptamer remains stable at around $-42 \mathrm{mV}$ throughout (Table A1). This comparison leads us to conclude that the 34-mer aptamer initially adopts its target binding stem-loop conformation on the AuNP surface, but over time, the aptamer may undergo significant structural rearrangement and exhibits behavior resembling single stranded sequence that adsorbs strongly to AuNPs but cannot interact with the target. The strong binding to AuNPs leads to imbalanced adsorption and desorption kinetics, and thus more densely packed aptamers over time, consistent with a more negative zeta potential.

The decreasing gap over time between the zeta potential values with and without meth confirm that these observations are directly linked to the suppression of the sensor function. It could be argued that diminished signal sensitivity over time is simply due to excess aptamer density accumulating, which means that AuNPs could remain stable even if some aptamers desorbed. While it is true that excess aptamers suppress signal sensitivity, the driving force towards higher surface density, and convergence over time with the randomized aptamer, is strong evidence that 
the increasing aptamer packing density is a result of stronger 'single-stranded' surface binding modes disintegrating the stem-loop structures needed for target recognition. The zeta potential data also suggests that in the dissociation AuNP sensor assay with adsorbed aptamers, an even shorter lag time before exposing the target would result in higher sensitivity.

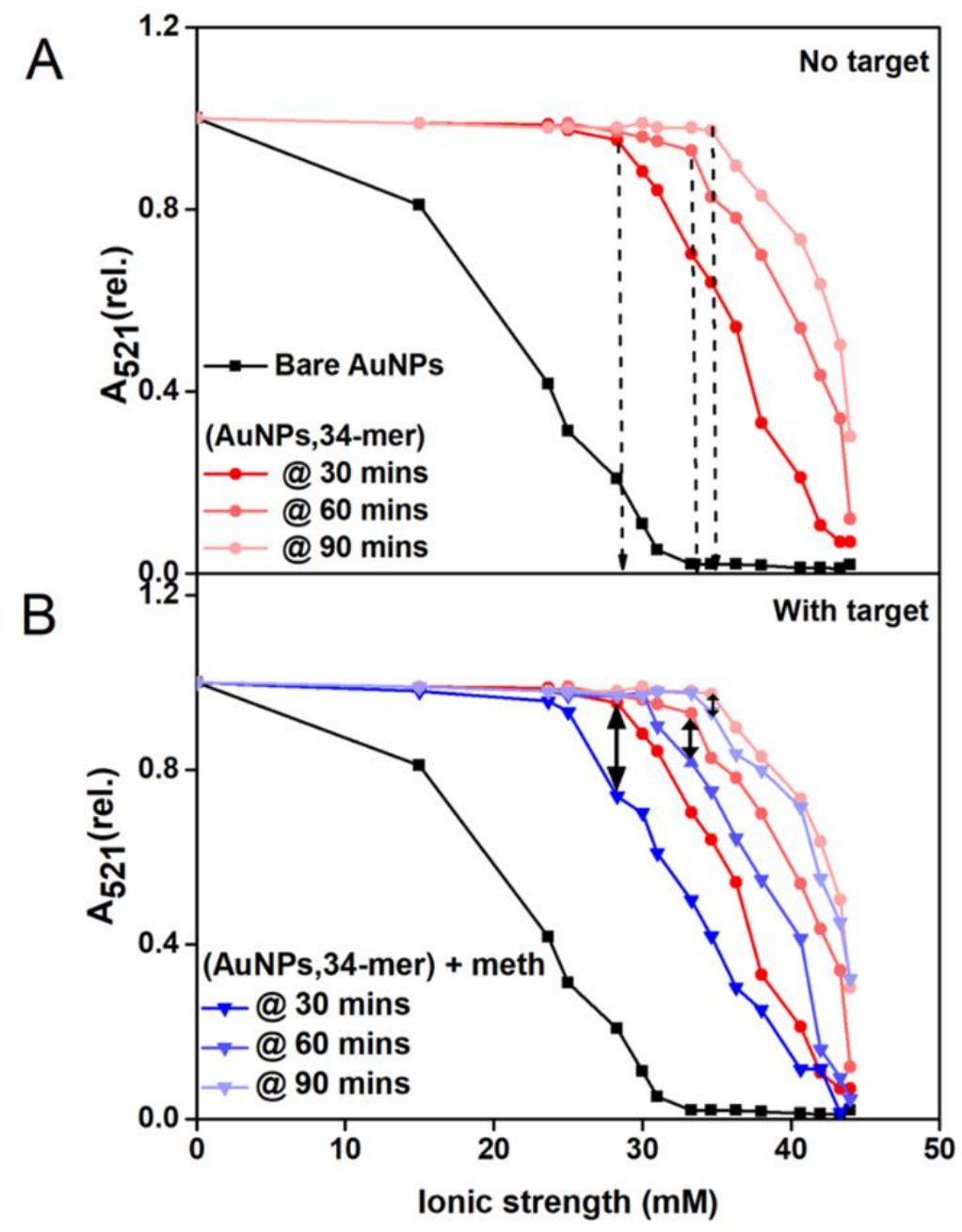

Figure 3.3 (A) Salt titration curve showing the optimal salt concentration shifting towards right as a function of time for the 34-mer meth binding aptamer. (B) Salt titration curve with target showing the decreased sensitivity towards meth over time. 


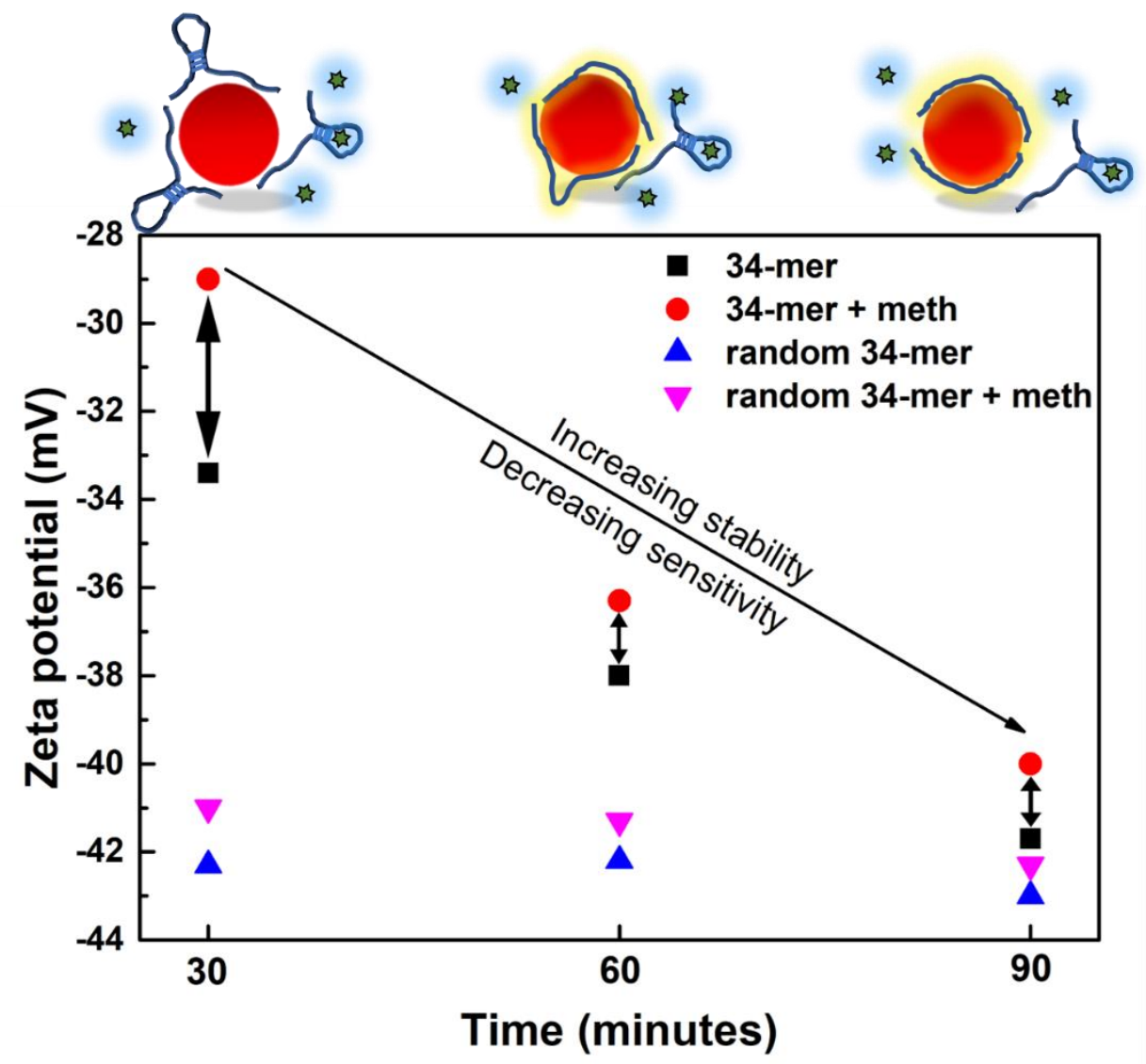

Figure 3.4 Plot of zeta potential vs time showing the increasing stability of aptamer adsorbed AuNPs (3:1) and decreasing sensitivity in the presence of meth over time. The top panel shows the proposed hypothesis.

Having established that the aptamers become less sensitive over time to the presence of meth while adhered to AuNPs, it was hypothesized that this problem could be circumvented by changing the sequence of mixing AuNPs, aptamers, and meth. In the new format - the so called 'aptamer association assay'- rather than exposing aptamers to the target when the aptamers are already adsorbed on AuNPs, instead it was ensured that the aptamers were first incubated with meth in solution, and then add AuNPs, followed by salt. In this way, the aptamers that do not bind meth will preferentially adsorb on the AuNPs, and a greater extent of aggregation and color change may be observed when meth-bound stable complexes cannot adsorb to the AuNPs.

\section{Colorimetric sensing using association assay}

The optimum ionic strength for the aptamer association assay format was found to be $28 \mathrm{mM}$ which is less than for the adsorbed assay format $(30 \mathrm{mM})$. This difference might seem modest, but 
it is instrumental in obtaining an optimal sensor response. The lower salt concentration required to destabilize the AuNPs in the aptamer association assay is consistent with a lower surface coverage of aptamers when meth-bound aptamers remain in solution, consistent with our idea that aptamer deactivation on AuNP surfaces.

Figure 3.5B shows that the colorimetric sensing response of the aptamer association assay is superior to the standard dissociation assay. The lowest level of meth that the aptamer association assay can detect is $20 \mathrm{nM}$ spectrophotometrically and $200 \mathrm{nM}$ colorimetrically with a linear detection range of $20-200 \mathrm{nM}$ and $\mathrm{R}^{2}=0.97$. The calculated statistical LOD was $33.3 \mathrm{nM}$ which was lower than that observed for the dissociation assay format $(81.2 \mathrm{nM})$. It was observed that by exposing the aptamer towards target first before exposing to AuNPs, there is a remarkable enhancement in the nanomolar level detection response and a visible improvement in the naked eye detectable color change when compared to the aptamer dissociation assay (Figure 3.5A). Through our colorimetric experimental results, it was illustrated that the order of addition of AuNPs (whether AuNPs-aptamer exposed to target or target-aptamers exposed to AuNPs) determines the extent of visible response (color change) obtained. Based on our observation we may assume that this difference in the two assays is significant because the aptamer is unable to undergo further conformational change to form the necessary binding pocket for accommodating the small molecule meth while adsorbed to AuNPs. This also highlights the importance of investigating the specific interactions between AuNPs and aptamer as well as between aptamer and targets extensively for developing high performance colorimetric sensors. The enhancement in response obtained also helped us to determine that the aptamer is more sensitive to the presence of meth while it is free in solution than while adhered to AuNPs. The LOD may be even lower when lower aptamer coverage is used, however it was found that low aptamer coverage is compromised by worse reproducibility. 

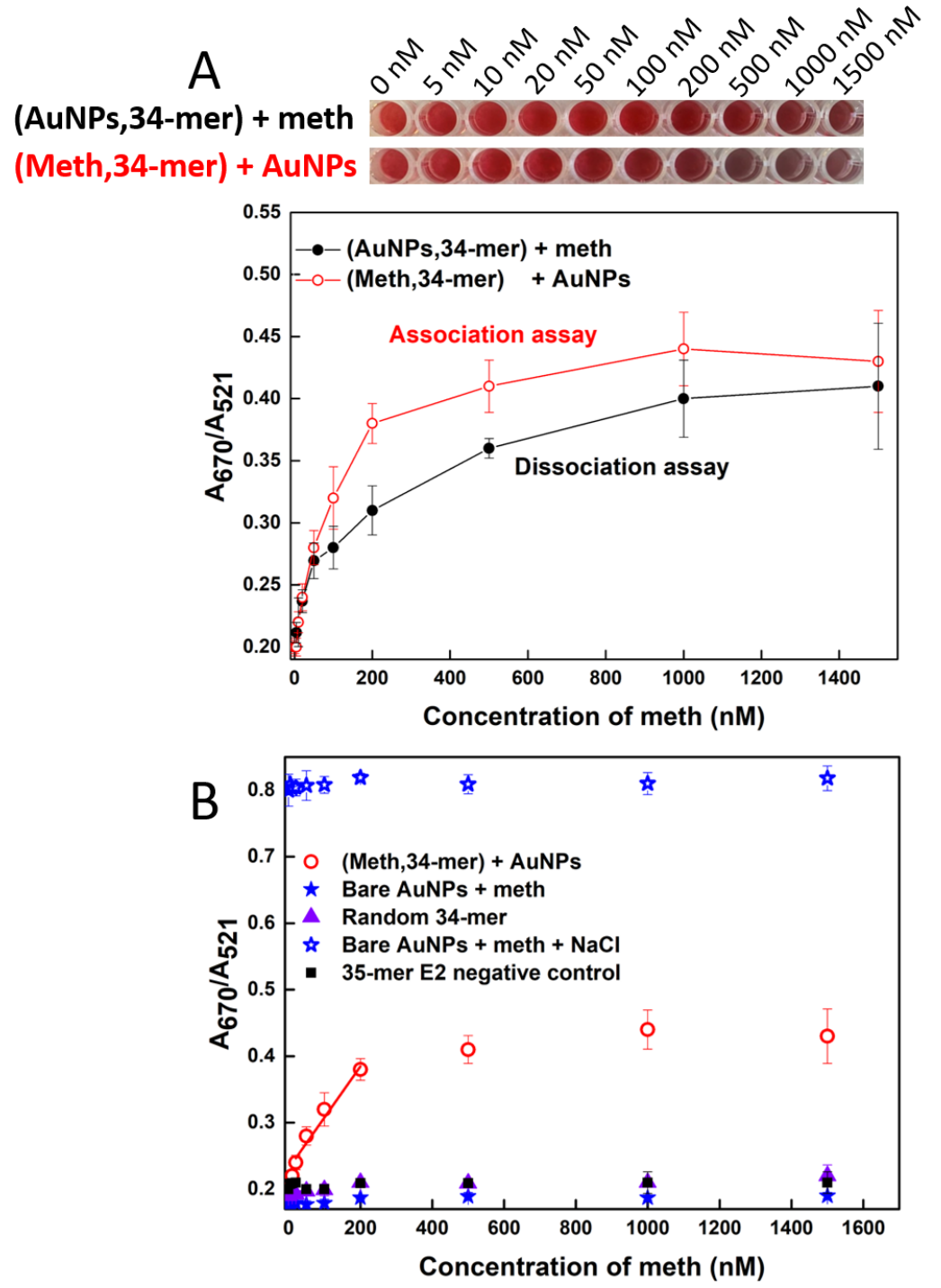

Figure 3.5 (A) Colorimetric aptasensors response comparison for the 34-mer meth binding aptamer using the aptamer association assay vs dissociation assay. (B) 34-mer aptamer association assay response along with bare and random 34-mer controls and inset showing the linear fit. Error bars represent standard deviation of the mean of three measurements.

A summary of responses of the meth binding aptamers and comparison of the two assay formats can be seen in Figure A12. It was concluded that an enhancement in the detection sensitivity is due to increased opportunities of binding between the aptamer and meth, which was previously hindered by kinetic trapping of aptamers in inactive forms on AuNP surfaces. In the association assay, not only can the aptamers interact with the target before the AuNPs are introduced, the time that the aptamers contact the AuNPs is also reduced. The LOD ultimately achieved is promising 
when compared to other AuNPs based colorimetric methamphetamine sensors reported in literature, as shown in Table 3.2. ${ }^{175,176}$

Table 3.2 Comparison between sensors for methamphetamine detection reported in literature and present work

\begin{tabular}{|l|l|l|l|l|}
\hline Serial no & Detection method & Strategy & Detection limit & $\begin{array}{l}\text { Matrix } \\
\text { detection }\end{array}$ \\
\hline 1 & Visual/colorimetric $^{177}$ & $\begin{array}{l}\text { AuNPs aggregation } \\
\text { assay }\end{array}$ & $0.82 \mu \mathrm{M}$ & Urine \\
\hline 2 & Colorimetric $^{178}$ & $\begin{array}{l}\text { AuNPs aggregation } \\
\text { assay }\end{array}$ & $\mu \mathrm{M}$ range & Water \\
\hline 4 & Electrochemistry & & \\
\hline 5 & Colorimetric (present work) & AuNPs aggregation & $33.3 \mathrm{nM}$ & Buffer \\
\hline
\end{tabular}




\section{Specificity investigations using association assay}

The specificity of the aptamer towards meth was evaluated by testing its response towards potential interferants of meth. Five closely related compounds namely amphetamine, pseudoephedrine, ephedrine, MDMA and MDA were chosen and the ratio of absorbances at $670 \mathrm{~nm}$ and $521 \mathrm{~nm}$ were recorded. As seen in Figure 3.6, the response of the aptamer towards meth is considerably stronger than the other compounds, but MDMA is found to exhibit some degree of interfering response. In order to rule out any non-specific interactions between the interfering molecules and AuNPs, bare AuNPs were incubated with each interfering molecule. It was observed that no nonspecific interaction was significant at the tested concentrations (Figure A8). It was also found that the sensor can selectively respond to meth in the presence of other interferants, which could be a useful feature for detecting a class of similar drugs.

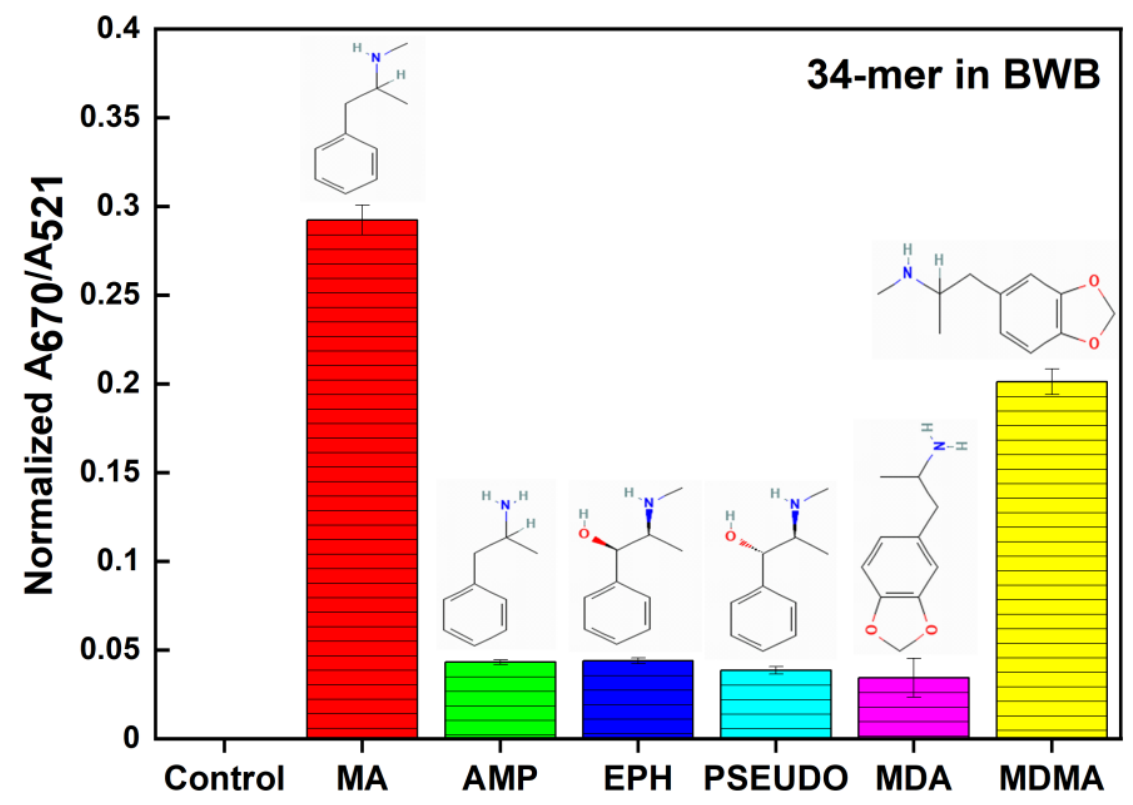

Figure 3.6 Specificity examinations of interfering molecules methamphetamine (MA), amphetamine (AMP), ephedrine (EPH), pseudoephedrine (PSEUDO), 3,4-methylenedioxyamphetamine (MDA), 3,4methylenedioxymethamphetamine (MDMA) at $200 \mathrm{nM}$ in BWB. Normalized absorption ratios were generated by subtracting the blank value. Error bars represent standard deviation of the mean of three measurements. 
Table 3.3 Summary of main aptamer sequences used, assay formats used and LOD achieved

\begin{tabular}{|l|l|l|l|}
\hline Aptamer sequence ID & Length & Assay format & LOD \\
\hline ABL Met S-03 & $75-\mathrm{mer}$ & Dissociation assay & $240 \pm 3 \mathrm{nM}$ \\
\hline Truncated Met S-03 & $34-\mathrm{mer}$ & Dissociation assay & $81.2 \pm 3 \mathrm{nM}$ \\
\hline ABL Met S-03 & $75-\mathrm{mer}$ & Association assay & $200 \pm 2 \mathrm{nM}$ \\
\hline Truncated Met S-03 & $34-\mathrm{mer}$ & Association assay & $33 \pm 5 \mathrm{nM}$ \\
\hline
\end{tabular}

\section{Complementary characterization techniques to gain qualitative insight into the nature of binding}

\section{$C D$}

CD studies showed that there was no significant change in the CD spectrum of 34-mer aptamer in the presence of target methamphetamine (Figure 3.7). This observation was not surprising because it is well known that the conformational change induced by a small molecule target is not always significant unless the aptamer is forming a well-defined three-dimensional structure upon target binding such as a G-Quadruplex. ${ }^{179}$ Small molecule targets may be groove binders or intercalators depending on their mode of interaction and it is rarely possible for groove binding small molecules to induce any conformational change in the DNA structure and hence these interactions are challenging to analyse using CD. ${ }^{180}$ Therefore to investigate further, it was necessary to look at the ITC profile the interaction between the aptamer and meth. 


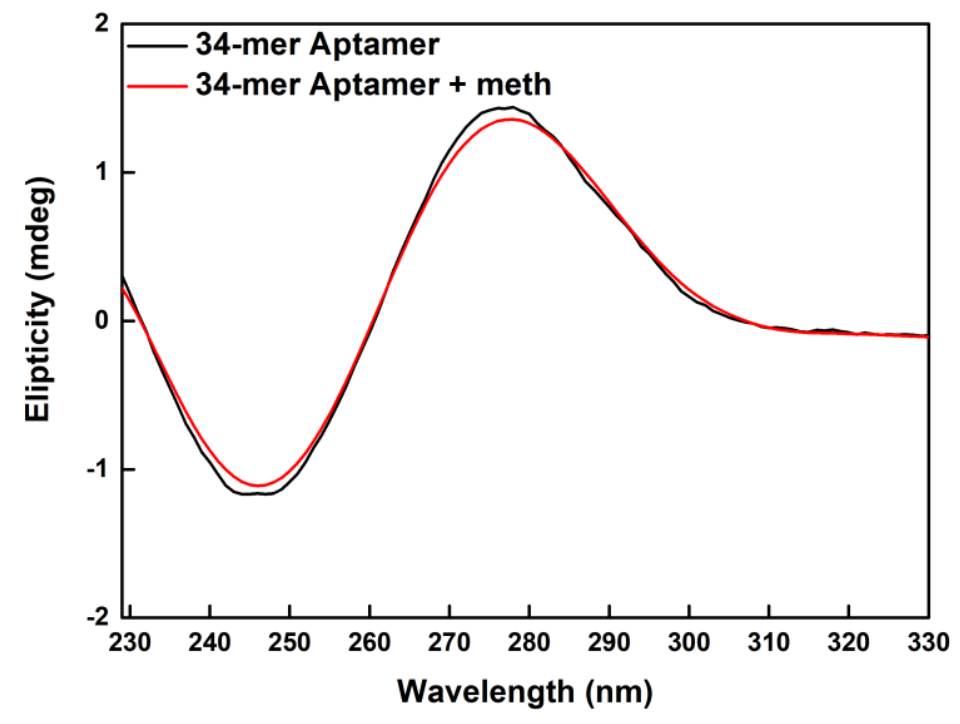

Figure 3.7 CD experimental data for 34-mer meth aptamer alone and in the presence of meth show the characteristic B-form ellipticity peaks at $279 \mathrm{~nm}$ (positive) and $249 \mathrm{~nm}$ (negative) suggesting no conformational change in the structure of aptamer induced by meth.

Therefore, it was important to investigate the binding via ITC to get a qualitative insight into the nature of binding and also, it may serve as a complementary evidence for proving a binding event.

\section{ITC}

The ITC was able to detect heats of binding as shown in Figure 3.8. When substances bind heat is absorbed or generated. Depending on whether the molar enthalpy is negative or positive, it is possible to say whether a reaction is exothermic or endothermic respectively. Each peak in an ITC thermogram represents the heat change associated with binding which happens after each injection of a small volume of titrant. It was possible to get a binding curve however, the experiment may not have been perfectly optimized due to which quantitative information such as the stoichiometry of binding given by the n-value may be unreliable. Moreover, the Kd obtained $(\sim 35 \mu \mathrm{M})$ and the n-value $(\sim 9.5)$ is an indication of high nonspecific interaction taking place between meth and aptamer at the high concentrations used during ITC. Since meth is a small molecule it is possible for the meth to interact with the aptamer non-specifically at places other than the binding domain. Therefore these values were not indicative of the specific complex formation and cannot relate to the Kd value found using Fluorescence assay $(\sim 200 \mathrm{nM})$ or the LOD $(\sim 33.3 \mathrm{nM})$ obtained using 
the colorimetric assay where the concentration of aptamer and target were at far lower concentrations. Hence, for finding a reliable $\mathrm{Kd}$ for this particular system, the ITC was not a very suitable technique because it did not reflect how the sensor actually worked. However, it is possible to discuss the other parameters deduced from the binding such as the nature of interactions. The ITC suggests that the interaction between the target and aptamer is largely entropically driven as suggested by the high positive entropy value, which is very typical of many small molecule's interaction with DNA aptamers. 

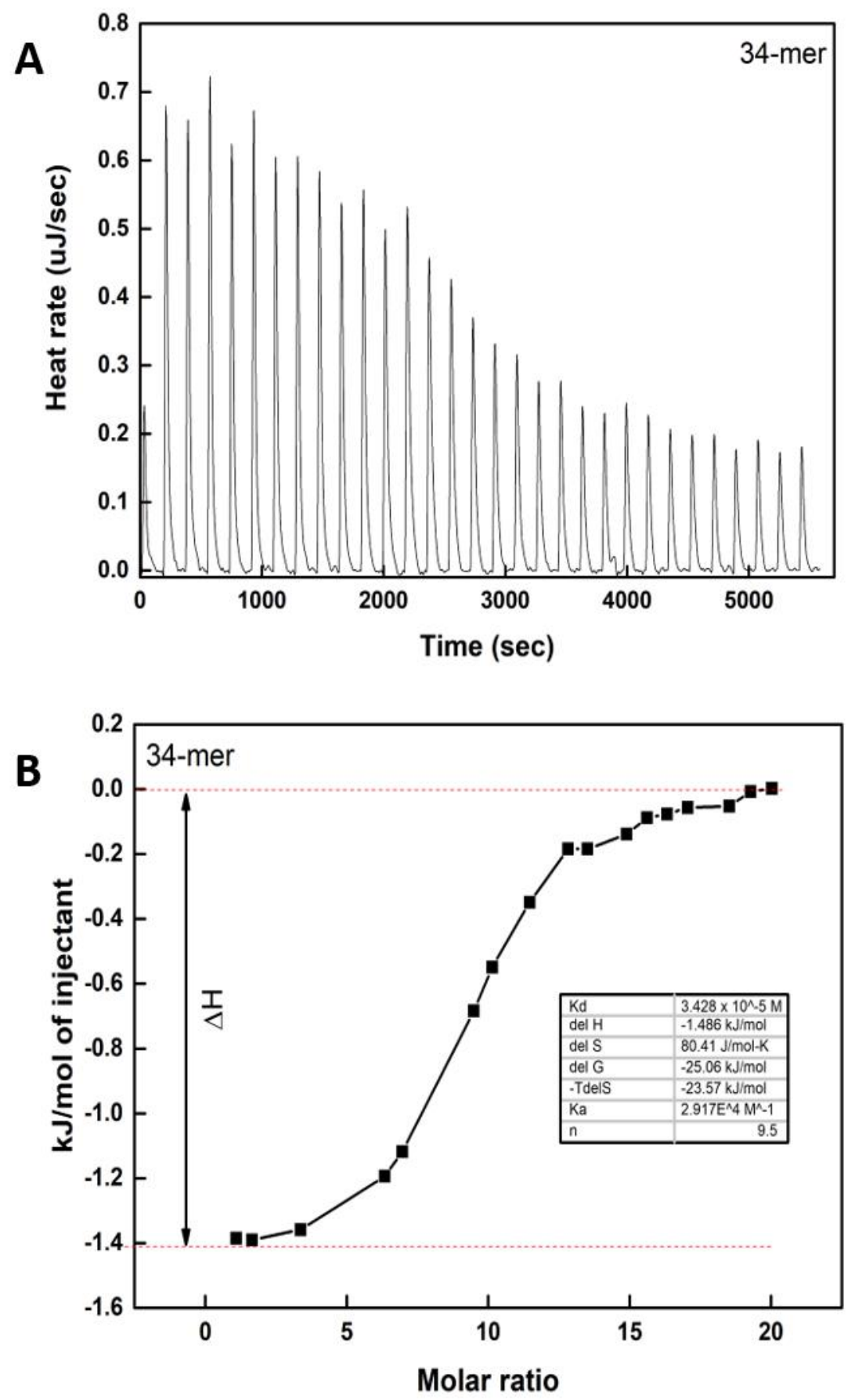

Figure 3.8 (A) Raw ITC thermogram for the 34-mer aptamer and meth interaction and (B) the integrated heat profile of the 34-mer aptamer interaction with meth. The data shown is with the blank value subtracted. 
The enthalpy is very slightly negative which suggests that there is no large conformational change taking place on binding to target, because the enthalpy change depicts the amount of heat energy required to attain a particular state signifying a conformational change involving hydrogen bond formations. Large, favourable negative enthalpy values are common for binding reactions but not omnipresent. ${ }^{150}$ On the other hand entropy is a measure of how the energy is distributed among various energy levels. Positive entropy is also a common characteristic of binding reactions.

Here, the values of entropy and enthalpy obtained strongly suggests groove binding as the mechanism of binding between meth and 34-mer aptamer which are always entropically driven and involves no obligatory conformational change in the B-form DNA structure. ${ }^{150,181}$ Entropy signifies disruption of order which is mainly caused by the disruption of solvation interactions resulting in the release of water and signifies hydrophobic interactions. In the ITC data it can be seen that the binding interaction between the aptamer and small molecule target is entropically driven signifying non-covalent interactions but it is very challenging to exactly attribute the contribution of different non-covalent interactions such as electrostatic, hydrogen bonding, solvation and hydrophobic interaction, Van-der Waals interaction. It could be a combination of all these non-covalent interactions leading to a binding interaction between the aptamer and small molecule, but certainly no significant conformational change takes place in the aptamer conformation involving bond formation induced by the target. As proposed by Freire et al. ${ }^{182,183}$ binding enthalpy may be used to signify bond formation involving hydrogen and van-der Waals bonding suggesting specific binding and whereas an entropically driven interaction may signify non-specific hydrophobic interactions.

The existence of non-specific hydrophobic binding interactions is not very surprising for small molecule aptamer development. Especially when using affinity-based SELEX methods to develop aptamers for small molecules. Because for small molecule aptamer development it is very challenging to avoid non-specific interactions between small molecules and aptamer (which contribute largely to non-specific affinity) except in rare cases, the focus should be on achieving specificity over affinity. These findings are well in line to explain the CD results.

Moreover, another possibility which may be discussed is the chances of non-specific electrostatic interactions with the highly negatively charged backbone of the DNA aptamer which could be accompanying the hydrophobic binding interactions as well. It may be possible that this could be 
overcome by using a high ionic strength buffer since that may help to stabilize the negatively charged backbone of the DNA and screen the possibility of non-specific interactions between meth and DNA backbone. However, more investigations may be required to discuss further about this. Based on the information from the ITC, it is possible to propose that the non-covalent interactions between the binding pocket of aptamer and meth may be hydrophobic in nature indicated by the large positive (favourable) entropy value which is characteristic of desolvation driven by hydrophobic interactions and very negligible formation of hydrogen bonds suggested by the low negative enthalpy value. The Gibbs free energy value which is a result of the opposing enthalpy and entropy contributions is negative and favourable suggesting the reaction takes place spontaneously.

Finally, having proven that aptamers become inactive over time on AuNP surfaces, it was considered whether aptamer selection strategies might adapt to deliver aptamers that are better suited to particular sensing formats. The parent aptamer was selected by a standard affinity-based SELEX procedure, in which aptamer sequences with the highest affinity to the immobilized target are amplified. However, it was shown here that eliciting a sensing signal in the colorimetric AuNP assay also requires strong consideration of the AuNP-aptamer interactions occurring in competition with target binding. Hence, it was concluded that alternative SELEX strategies that complement the desired sensor format should be developed, which impart evolutionary pressure towards aptamers with the properties that will perform best in a sensor platform. For example Kim et al. and others demonstrated the importance of the effect of screening method on the selection of aptamers by developing an immobilization free reduced Graphene Oxide-SELEX to select a novel ss-DNA aptamer sequence against glycated human serum albumin (GHSA) free in solution and found enhanced sensitivity (in nanomolar range compared to micromolar ranges reported previously for GHSA) and specificity of the aptamer towards target when compared to other aptamers selected via unmodified SELEX. ${ }^{184}$ Also, AuNP SELEX for developing aptamers for small molecules such as brassinolide and bisphenol have been reported which could be extended for developing more sensitive, specific and conditioned aptamers for meth in the future. ${ }^{75}$ 


\subsection{Chapter conclusion}

Whereas colorimetric AuNP aggregation assays assume an equilibrium competition between aptamer adsorption to AuNPs and binding to target molecules, it was demonstrated here that aptamer-AuNP interactions are in fact time dependent. Over time, aptamers become more tightly bound to AuNP surfaces and less available to recognize and bind to target molecules, thus rendering the sensor less effective. Having illustrated this phenomenon via time-dependent zeta potential and salt stability assays, a modified assay to circumvent the effect of inactive aptamer structures becoming trapped on AuNP surfaces developed. Instead of probing aptamer-target interactions via the target-induced dissociation of metastable aptamer structures from AuNPs, free aptamers were exposed to the target before allowing association with AuNPs. By inverting the sequence of steps, as well as eliminating excess bases from the sequence, it was demonstrated that an 8-fold improvement in the level of detection/sensitivity for a methamphetamine aptasensors was achieved. Although our approach that accounts for time-dependent aptamer-AuNP interactions offers significant improvements, it also reveals shortcomings in the typical way that SELEX is used for aptasensors. SELEX evolves aptamers with high affinity for the target, without regard for how downstream signals will be transduced. In the case of colorimetric AuNP aggregation assays, aptamers would ideally be pre-conditioned to maintain dynamic equilibrium between free and adsorbed states that remain available to interact with the target. Moreover, CD studies show no significant conformational change taking place on target binding which suggests that the degree of structural rearrangement induced by the target is quite insignificant and the ITC studies support the idea of groove binding mechanism between aptamer and meth which is consistent with what CD shows. ITC data serve as a complimentary evidence to colorimetric assay results to support the binding interaction taking place between aptamer and meth and also sheds light on some thermodynamic parameters which help in knowing the mechanism and nature of binding interactions taking place. 


\section{CHAPTER 4}

AuNP SELEX generated aptamers for the detection of methamphetamine using AuNP aggregation assay 


\subsection{Abstract}

Modified SELEX strategies are becoming an emerging choice because they have been shown to enhance the performance of aptamers especially for small molecule targets. This motivated the application of AuNP SELEX selected aptamers for the detection of meth using AuNP aggregation assay. The problem of inactivated aptamers due to tight adsorption on the surface of AuNPs, demonstrated in Chapter 3 necessitated the investigation of an AuNP SELEX selected aptamer to further explore the hypothesis stated in Chapter 3. The findings not only demonstrate the credibility of the claims made in Chapter 3 but improved levels of detection as low as $10 \mathrm{nM}$ were obtained with the modified SELEX selected aptamer which indicated a 3-fold improvement in the LOD compared to traditional SELEX selected aptamer $(33 \mathrm{nM})$. Having demonstrated this, the success of using modified SELEX strategies that account for the downstream application of aptamers becomes clearer and may encourage the development of more successful aptamers for SMs detection. 


\subsection{Introduction}

As discussed in Chapter 1, traditionally, oligonucleotides with binding affinity to a molecule have been isolated via nucleotide library incubation with an affinity matrix that present a bead immobilized stabilized target molecule as shown in Figure 1.12, Chapter $1 .^{78,185}$ This restricts access of the oligonucleotides to the entire molecule, and in particular, the functional group(s) often used for conjugation purposes. The range of targets used in SELEX varies from proteins, immunogens, organisms, whole cells, small molecule etc. . $^{186,187,188}$

More recently, the focus indeed has shifted to in-solution methods of selection, isolating the library from solution rather than the target molecule. Especially for small molecule targets, switching to in-solution methods of selection have proved to generate aptamers with enhanced binding affinities as demonstrated previously by $\mathrm{Gu}$ et al. ${ }^{76}$ who developed non-immobilized graphene assisted SELEX procedure for developing aptamers with high affinity and specificity for Okdaic acid, a low molecular weight molecule and implemented the aptamer into a aptasensors platform for detection of OA in shellfish samples. Similarly, a reduced graphene oxide (rGO) based, in solution SELEX was developed to select aptamers that exhibited high specificity and sensitivity towards a glycated human serum albumin. ${ }^{184}$ The selected aptamer was then applied to a rGO based sensor platform for detection of GHSA with higher sensitivity and specificity when compared to other aptamers for GHSA screened through other methods. The work clearly demonstrates that the screening method determines the performance of an aptamer for downstream diagnostic purposes and such considerations deserve much focus. Stoltenberg et al. ${ }^{74}$ developed a special CaptureSELEX procedure to enable selection of DNA aptamers for solute SMs citing traditional SELEX as unsuitable for selecting aptamers for SMs.

However, small molecule targets such as methamphetamine present a unique challenge for aptamer selection using the traditional SELEX technology, as methods of selection require immobilization of the target molecule in order to enable removal of non-binding oligos from the library pool. These methods require utilization/occupancy of functional groups of the target molecule, which can reduce the affinity and specificity of the aptamer especially in case of SMs which do not have multiple functional groups. Moreover, the immobilization process may alter the original conformation of the SM target and steric hindrance may mess with the aptamers binding site, all leading to generation of aptamers with compromised specificity. 
Because of all these shortcomings, it was necessary to investigate alternative or modified SELEX strategies which would not only facilitate selection of aptamers for SMs free in solution but also be compatible with the aptamer's downstream application for example it's translation into a sensor platform. Therefore, the SELEX group from the School of Biological Sciences department developed an in-solution method of generating aptamers for methamphetamine. The method utilized the nucleotide binding properties of AuNPs, and the ability of the target molecule to dissociate nucleotides that exhibit affinity to the molecule from the AuNP surface. The in-solution method was performed to generate an enriched library that was sequenced by standard plasmid ligation and cloning protocols, from which six aptamer candidates were randomly selected. They were characterized using an AuNP assay and ITC, against a comparator methamphetamine aptamer that was generated using traditional SELEX. The schematic of the AuNP SELEX can be found in Scheme 4.1.

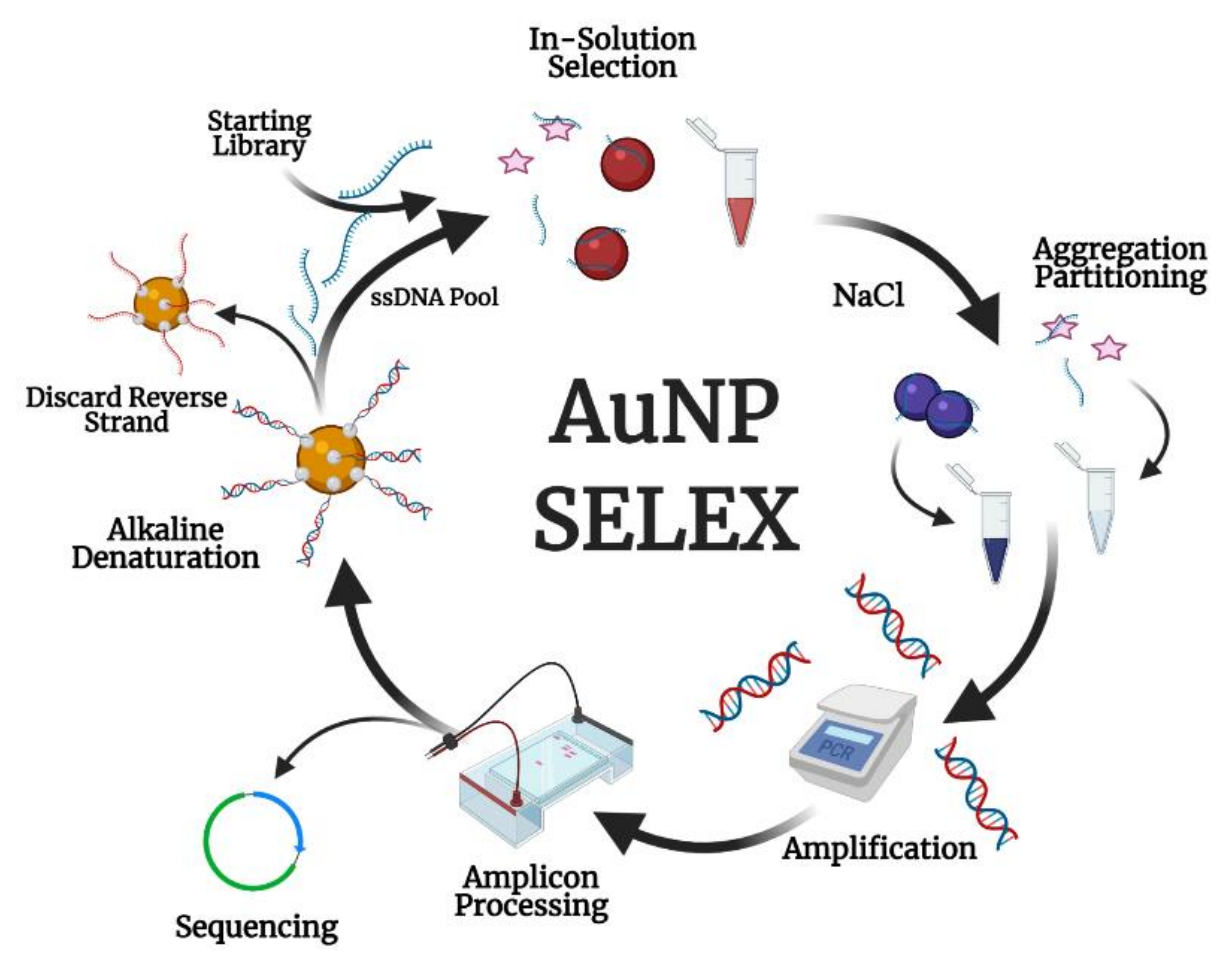

Scheme 4.1 Illustration of the AuNP SELEX procedure which uses in-solution selection of aptamers for small molecule targets that are non-immobilized and free in solution. This scheme was made by William Odey School of Biological Science as part of an unpublished work. 
In this work to further the proposition made in the previous chapter (Chapter 3), the effect of a modified SELEX strategy, the AuNP SELEX has been demonstrated. The generated aptamers that are pre-conditioned to match the AuNPs aggregation sensor platform were tested. It was hypothesized that this would result in higher performance owing to the reasons discussed above. Hence, new generation aptamer candidates selected were provided which were optimized and it was found that among the three potential candidates, a 81-mer aptamer sequence sensing response observed was remarkably promising compared to the 75-mer long counterpart selected via traditional SELEX. The findings help to prove our hypothesis regarding the effect of selection on downstream application of aptamers and circumvent the challenges associated with small molecule binding aptamers.

\subsection{Experimental section}

The materials and methods used were identical to those mentioned in Chapter 3. Both the association and dissociation assay were carried out to learn about the generality of the finding described in Chapter 3. All measurements with the 34-mer aptamer were repeated when comparing the response with the new aptamers in this chapter. It must be noted that, absolute values of the response cannot be stressed upon owing to the nature of the aggregation assays including some unavoidable variations among AuNPs batches (which is accounted for with the salt optimization steps). Therefore, it must be noted that the focus should be on consistency of trends in responses rather than on the absolute ratio of aggregation values. This is because the absorbance values at $670 \mathrm{~nm}$ is prone to changes.

\subsection{Results and discussion}

\section{Initial trials with AuNP SELEX generated aptamer candidates}

Initial trials were carried out to record responses of the new generation SELEX selected aptamers, OMC-9 81-mer and OMC-15 81-mer and compare them with that of the affinity-based SELEX selected aptamer that has been discussed in Chapter 3. The response of the new aptamer sequences was comparable to the affinity-based SELEX selected aptamer 75-mer. In this chapter, responses of the affinity-based SELEX selected aptamers will be discussed only for comparative purposes. 


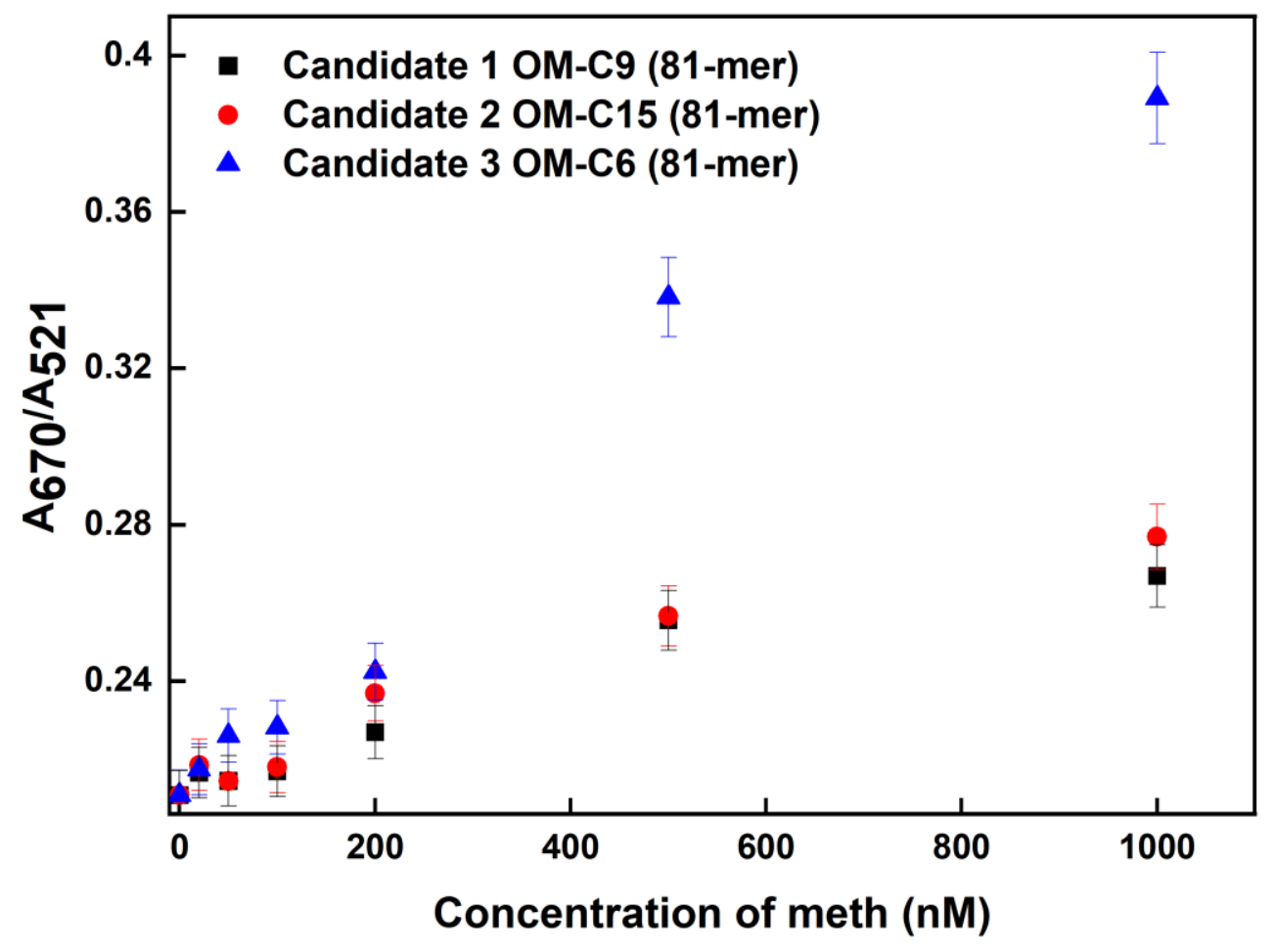

Figure 4.1 OM-C6 responds best among the other new generation SELEX developed candidates OM-C9 and OM-C15. Error bars represent the standard deviation of the mean of three measurements.

Subsequently, another aptamer sequence selected via the new generation SELEX was isolated and on recording the response of the aptamer, it was found that the response of OMC-6 81-mer or simply the 81-mer surpassed those of the previously tested new generation aptamers as shown in Figure 4.1 and therefore the 81-mer was selected to do further sensing studies. Interestingly it was found that the 81-mer aptamer's response using the colorimetric assay towards meth was far superior than the 75-mer and comparable to the truncated 34-mer which had been our best aptamer (in the previous chapter) until now. In the subsequent sections, the optimization and developement of the 81-mer aptamer has been discussed and it's response is discussed in comparison to the aptamer sequences discussed in Chapter 3. 


\section{Salt optimization and aptasensing with OM-C6 81-mer aptamer}

Figure 4.2 shows the salt optimization curve for the 81-mer aptamer. The optimal salt concentration was found to be $30 \mathrm{mM}$. The optimal salt concentration was found to remain unchanged on changing the sequence of addition (data not shown) and this was an important finding which led to further confirm the hypothesis proposed, discussed and investigated in Chapter 3 regarding the compromised dynamic equilibrium which needs to be maintained at the surface of the AuNPs. Moreover, it provides a better understanding of the importance and the effect of the screening/SELEX strategies on the performance of the aptamers during their downstream applications, which was also a concluding point of discussion proposed in Chapter 3.

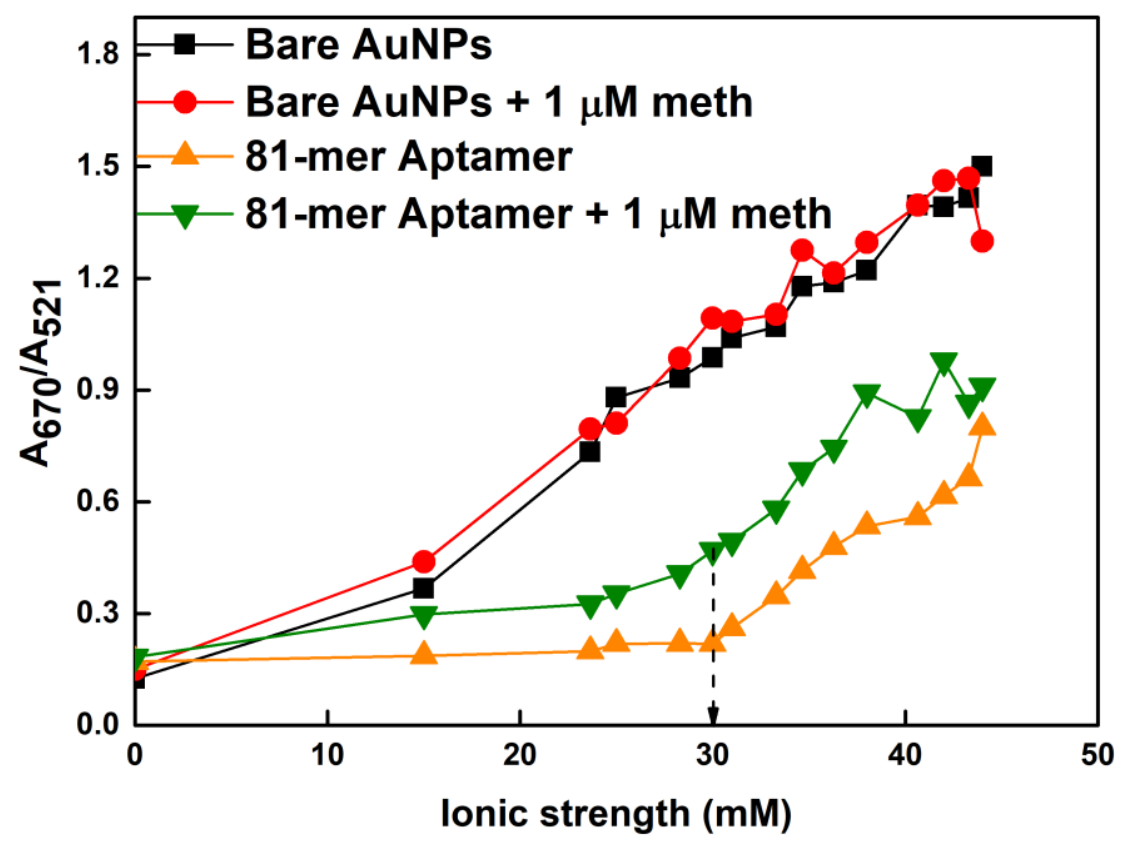

Figure 4.2 Salt optimization graph for the OM-C6 81-mer. The optimal salt concentration at $30 \mathrm{mM}$ is indicated by the dashed arrow.

The response of the 81-mer aptamer in comparison with 75-mer can be seen in Figure 4.3. AuNPsaptamer samples using the association assay for the 81-mer were mixed with various concentrations of meth $(0,0.1,0.5,1,2$ and $5 \mu \mathrm{M})$. the response recorded for the 75 -mer was with the dissociation assay because of reasons discussed in Chapter 3. This range covered the cut -off 
level for meth detection ( $167 \mathrm{nM}$ or $25 \mathrm{ng} / \mathrm{mL})$ and this also made sense because from previous experience, it has been shown that longer aptamers fail to detect low nanomolar concentrations of meth reliably. Therefore, low micromolar concentrations were used to begin with.

LOD for 75-mer was found to be $240 \pm 3 \mathrm{nM}$ and for 81 -mer it was $110 \pm 4 \mathrm{nM}$. This difference is quite similar to that observed between the 75-mer and 34-mer aptamers in Chapter 3, which suggested that the response of the 81-mer was comparable to the truncated 34-mer version of the 75-mer affinity based-SELEX selected aptamer. This was interesting because of two reasons, first it provided evidence that the modified SELEX strategy did help in generating better performing aptamers which satisfied the claims made in the previous chapter. Secondly, the response of the 81-mer motivated the investigation of truncated sequences which might perform even better and lead to high performance saliva sensor developement. 


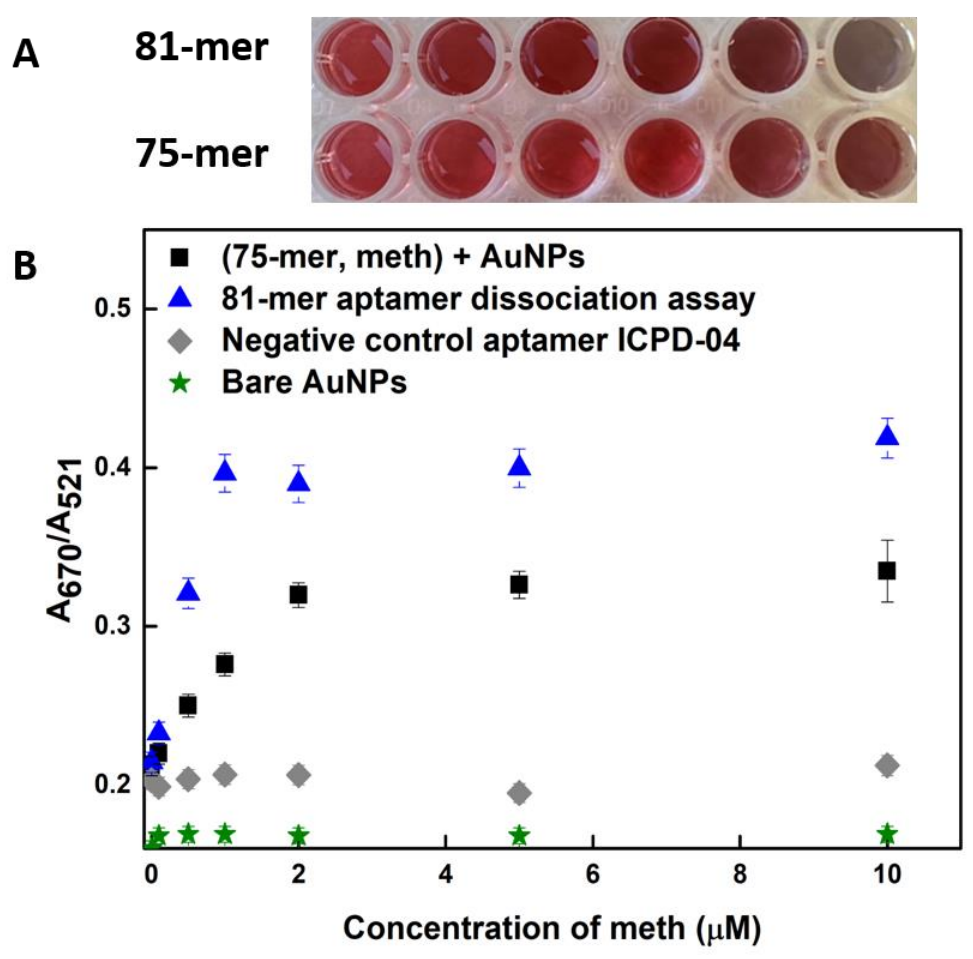

Figure 4.3 (A) Colorimetric sensing response of 75-mer and 81-mer aptamers with bare AuNPs and negative control aptamer ICPD-04. (B) Visual image of the same. The 75-mer response was recorded using association assay as dissociation assay was unsuitable for application as described in Chapter 3. Error bars represent standard deviation of the mean of three measurements.

\section{Truncation of the 81-mer aptamer}

Since the aptamer was selected using a modified selection strategy, the primers were not truncated like before because it seemed they might very well be a part of the binding domain (since they were very much part of the double stranded stem regions which may be considered as the probable binding region $)^{73}$ and not just present as redundant bases as witnessed earlier in Chapter 3 and in other works which have truncated primers off citing similar reasons. ${ }^{189,190,191}$ 


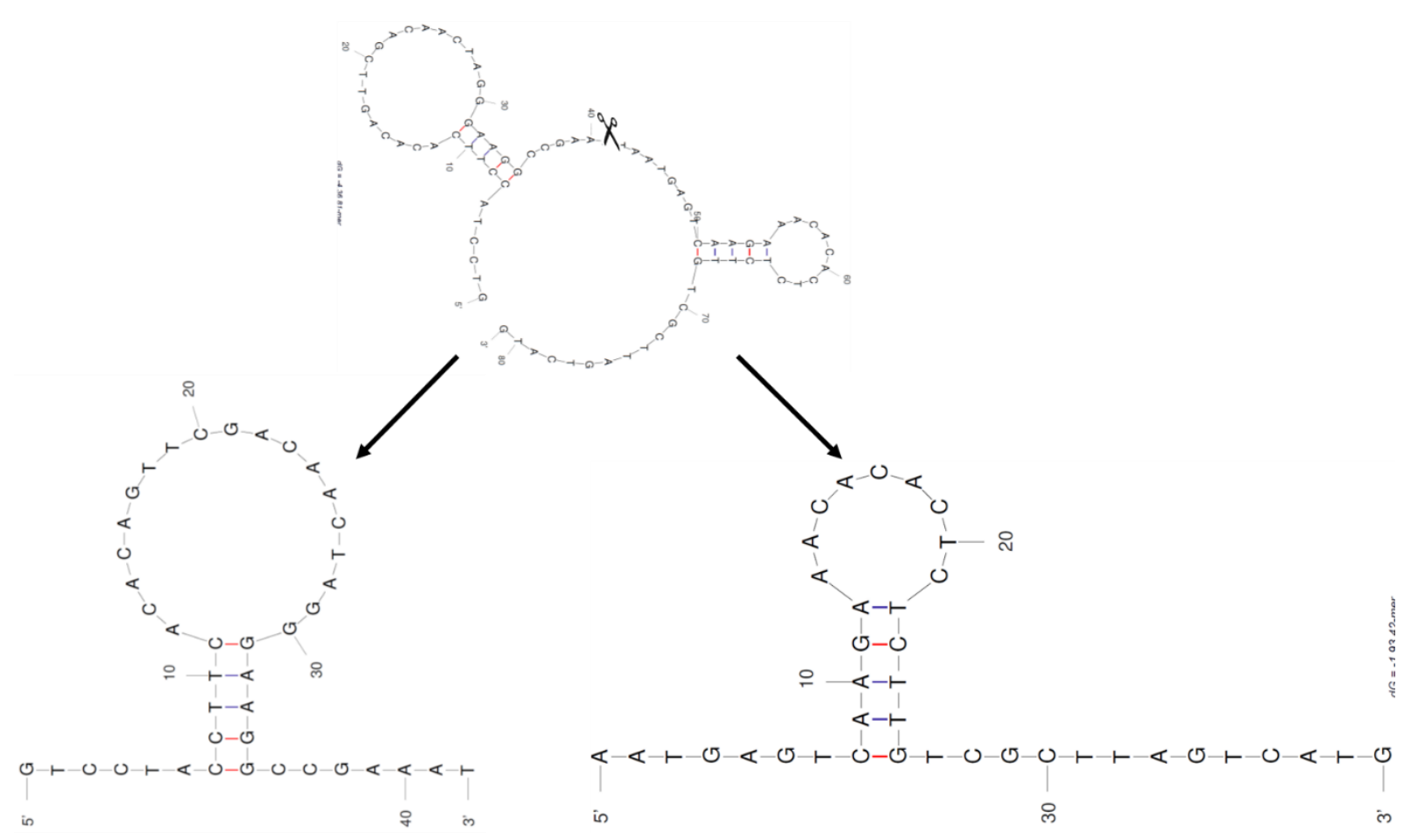

Figure 4.4 M-fold predicted structures of parent 81-mer and it's two halves constituting the intact double stranded probable binding regions, the 42 -mer and the $39-$ mer. The point of cut on the 81 -mer is represented by the black scissors.

Therefore, it made most sense to retain the primers as has opposed to what had been done before (refer to Chapter 3) but truncate the aptamer into halves and test both the double stranded regions to see which one of the two could be the main binding domain. On truncation a 42-mer and a 39mer sequence was generated as shown in Figure 4.4. It was found that both the halves responded to meth as shown in Figure 4.5. The response of the 39-mer was similar to that of the 81-mer and the 42-mer was clearly more responsive to meth suggesting that the main binding domain was comprised by the 42 -mer half. The interaction of the 39 -mer could be due to the meth interacting non-specifically or through cooperative interaction as has been the case shown previously through ITC studies and is quite prevalent for small molecule DNA binding aptamers. So, it may not be surprising to see that the meth could interact with the 81-mer aptamer because of the two potential binding domains. 


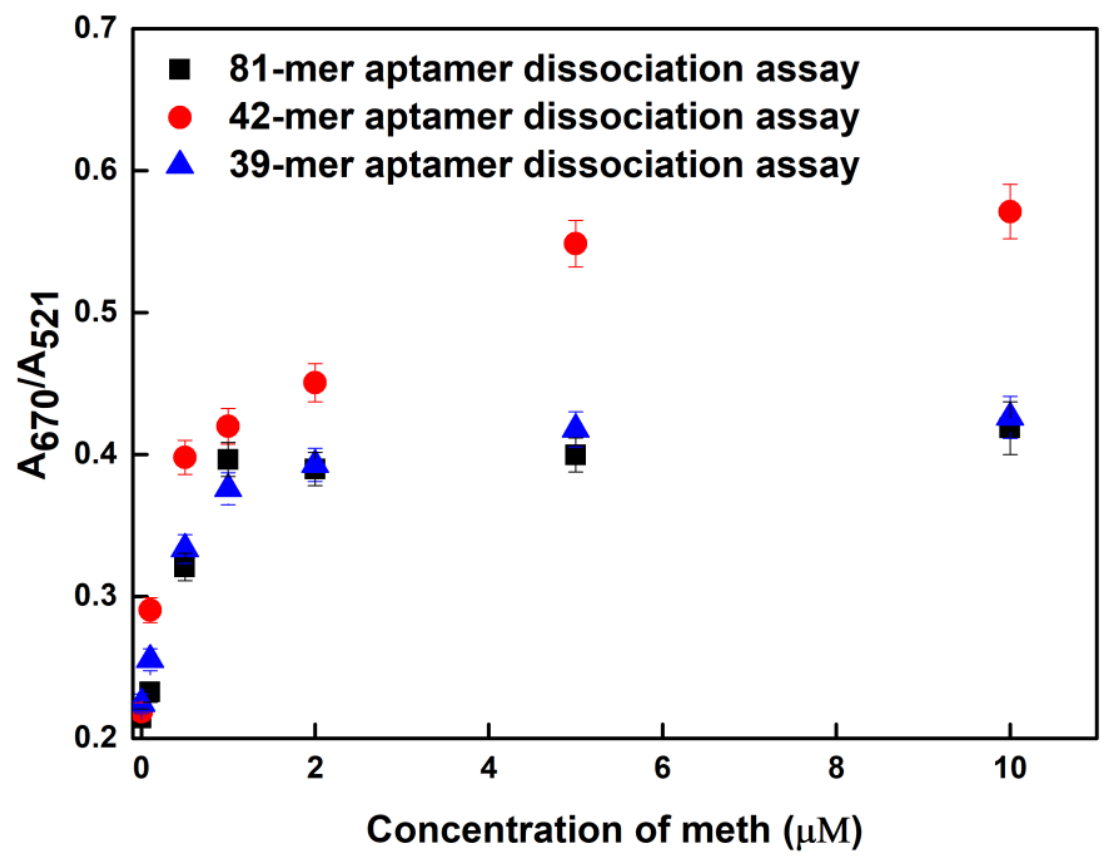

Figure 4.5 Colorimetric response of the parent 81-mer versus the two constituent halves, 42-mer and 39mer. The 39-mer was found to be similar to the 81-mer in its response and the 42-mer was clearly most promising and responsive towards meth. Error bars represent standard deviation of the mean of three measurements.

However, this would require further structural analysis such as native nano-electrospray ionization mass spectrometry (native ESI-MS), NMR and ITC to come to robust conclusions. ${ }^{192}$ However, this did not fall within the scope of this work.

\section{Aptasensing with the 42-mer truncated aptamer}

Since the response of the 42-mer was clearly stronger than the other half, therefore subsequent studies were carried out with the 42-mer sequence. The 42-mer surpasses in response to its parent aptamer and the 34-mer aptamer (Figure 4.8). We can clearly see the remarkable improvement in the detection response of the AuNP-based SELEX generated aptamers compared to the traditional SELEX generated aptamers. The optimal salt concentration of the 42-mer was determined to be $26 \mathrm{mM}$ as shown in Figure 4.6. In Figure 4.7, the sensing response has been recorded at a concentration range of $0-1000 \mathrm{nM}$ meth. The LOD of 42-mer aptamer was found to be $10 \mathrm{nM}$ 
which was a good improvement from the previously reported 34-mer aptamer with an LOD of 33 $\mathrm{nM}$. The colorimetric response of the 42-mer aptamer with inset showing the linear range can be found in Figure 4.7.

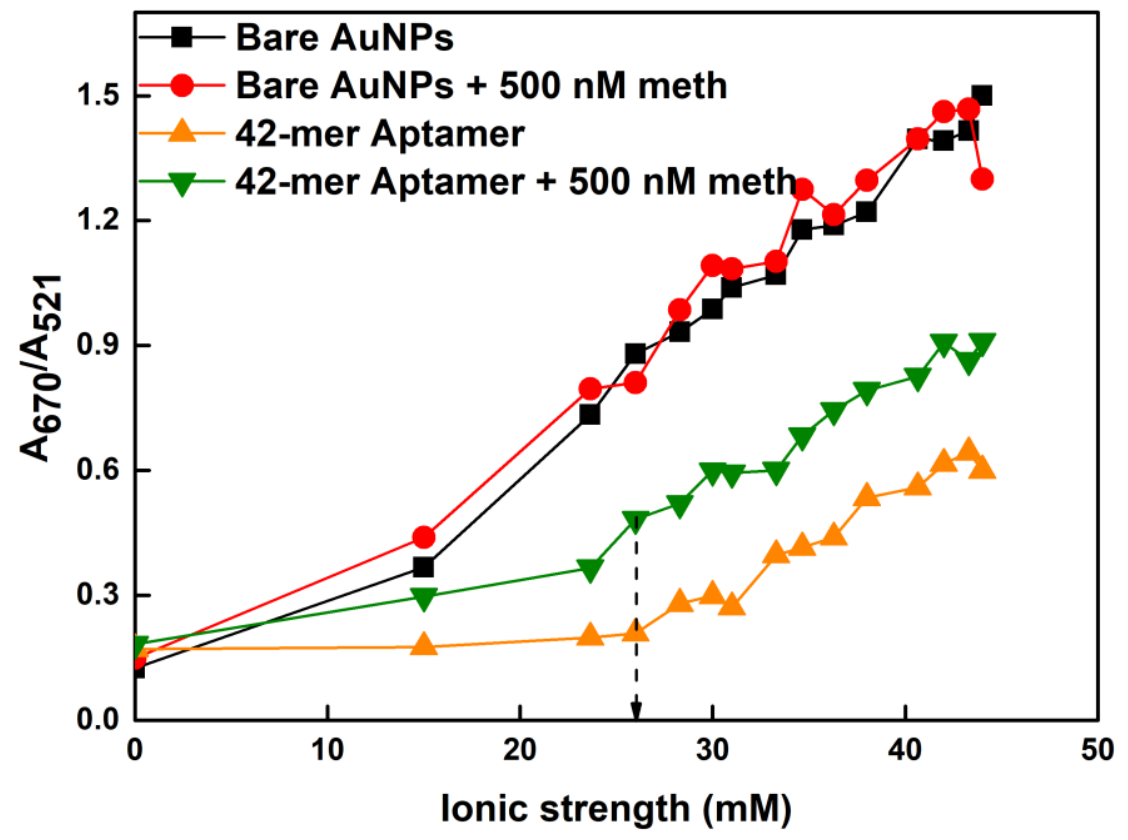

Figure 4.6 Salt optimization curve of the 42-mer. The optimal salt concentration was found to be $26 \mathrm{mM}$ represented by the dashed arrow. 


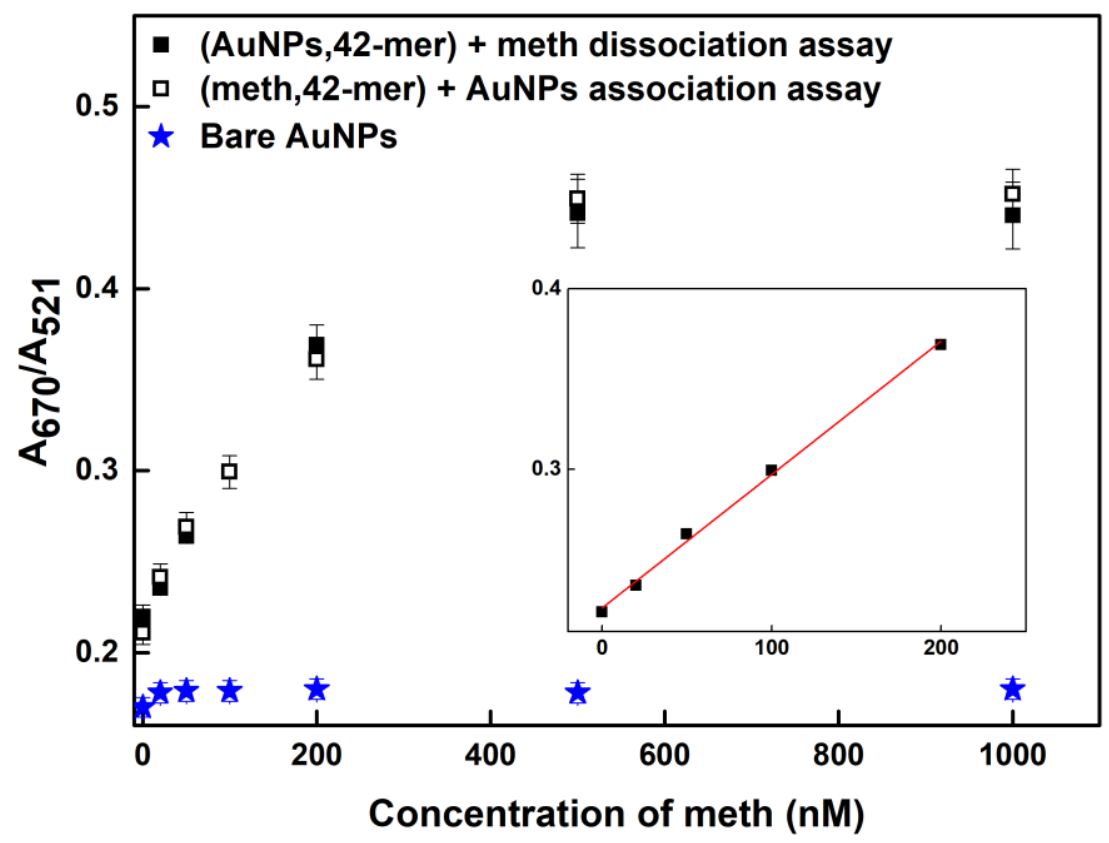

Figure 4.7 Colorimetric aptasensors response of the 42-mer towards meth using the association and dissociation assay to show the effect of the change of assay sequence on the response towards meth. Inset showing the linear range of the 42-mer aptamer from 0-200 nM. Error bars represent standard deviation of the mean of three measurements.

\section{Comparing traditional vs AuNP SELEX selected aptamers}

Although at low nanomolar concentrations there is not much difference in the response of the two parent aptamers (81-mer and 75-mer) because of depressed sensitivity brought about by excess redundant bases (Figure 4.8), the difference in their response is very evident when comparing the two sequences at micromolar concentrations of meth (Figure 4.3). We see that the new generation sequence is much more sensitive to meth in solution probably because of the selection procedure involved which not only pre-conditions the aptamer to retain sensitivity towards meth in the presence of AuNPs but also trains it to identify meth free in solution which is a major advantage of the modified SELEX method. Normally, SELEX procedures used to develop aptamers for small molecules use a target immobilized method which is the general method of affinity based SELEX, however this has often been discussed to be a major point of concern especially in case of small 
molecules because of the chances of compromising the target identifying ability of aptamers when applied to sensors for detection of SMs existing free in solution.

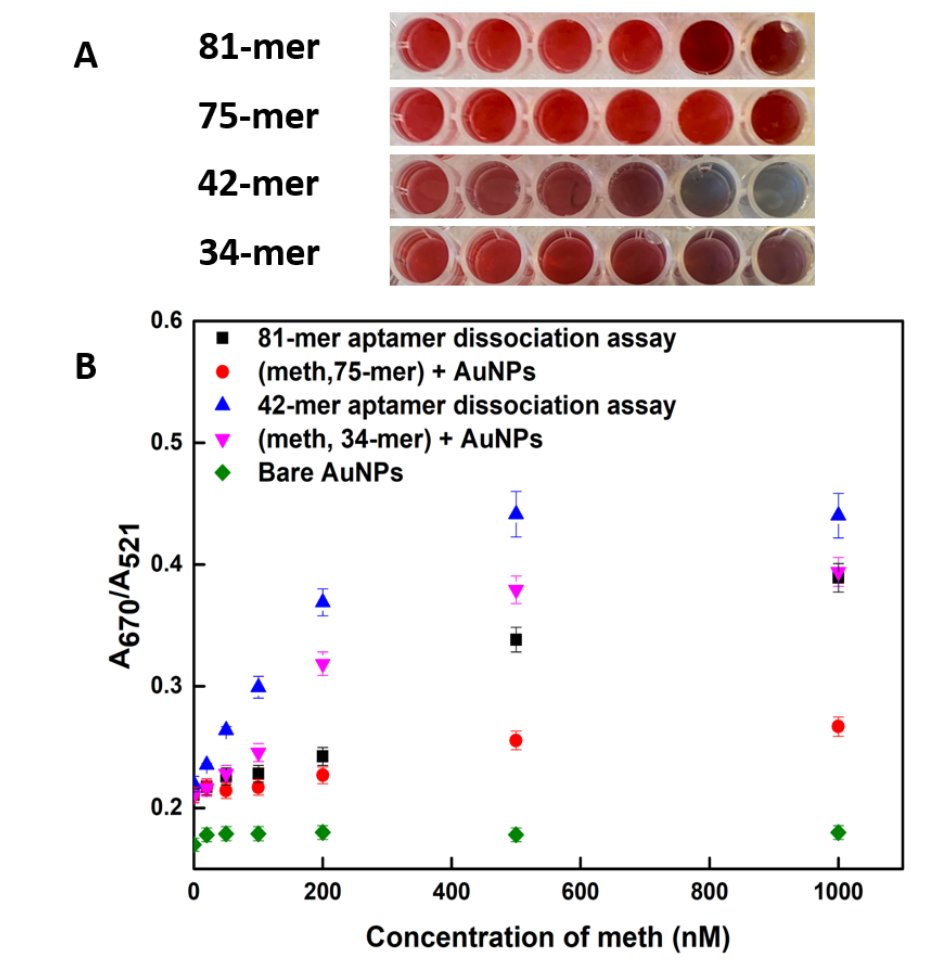

Figure 4.8 (A) Summary of colorimetric responses of the affinity-based SELEX selected aptamers 75-mer and truncated 34-mer and the new generation SELEX selected aptamers 81-mer and truncated 42-mer. (B) Visual image of the same. Error bars represent standard deviation of the mean of three measurements.

The comparison between the LOD's of 34-mer and 42-mer clearly demonstrates the increased efficiency in performance of the modified SELEX selected aptamers. Moreover, the fact that the assay could be carried out following the association assay since there was no significant difference between the two assay formats as has been previously reported in Chapter 3, was an important finding. It provided further knowledge about the problem of aptamers not pre-conditioned to associate and dissociate from AuNPs before identifying target. Moreover, comparison of the optimal salt concentrations for the two parent aptamers 75-mer and 81-mer say volumes about the nature of interaction with the AuNPs. The 81-mer aptamer being longer has a lower optimal salt concentration which demonstrates the fine balance needed to remain adsorbed to the AuNPs and also easily desorb in the presence of target without getting trapped on AuNPs surface. This fine balance may be a result of the selection process which selected target binding aptamers based on 
their ease of adsorption and desorption. Plus, the findings address the problem of identifying targets free in solution (during sensing assay) which may be confusing because of the exposure of aptamers to immobilized targets having extra chemical groups during the traditional SELEX process. These reasons and evidences could be satisfied and addressed by comparing the two aptamers for the same target but selected via different SELEX procedures and helped in advancing the knowledge about while demonstrating the effect of SELEX procedure on the downstream application of aptamers.

Based on the observations it may be concluded that the dependence of the assay response on sequence of addition of AuNPs was not a universally applicable strategy, but it is always worth investigating the assay response by changing the sequence of addition to get the best optimized system for the analyte of interest.

Table 4.1 Summary of main aptamer sequences used, their characteristics and LOD achieved

\begin{tabular}{|l|l|l|}
\hline Aptamer sequence ID & Characteristics (length \& selection) & LOD achieved \\
\hline OM-C6 & 81-mer, AuNP SELEX & $110 \pm 4 \mathrm{nM}$ \\
\hline ABL Met S-03 & 75-mer, traditional SELEX & $240 \pm 3 \mathrm{nM}$ \\
\hline Truncated OM-C6 & 42-mer, AuNP SELEX & $10 \pm 3 \mathrm{nM}$ \\
\hline Truncated Met S-03 & 34-mer, traditional SELEX & $33 \pm 5 \mathrm{nM}$ \\
\hline
\end{tabular}

\section{CD investigations}

CD studies showed that there was no significant change in the CD spectrum of the 42-mer aptamer in the presence of target methamphetamine (Figure 4.9) which was similar to what was found with the 34-mer aptamer in Chapter 3. This indicates that there was no significant structural change on target binding that could be determined by the CD. As mentioned in Chapter 3, the mode of interaction of meth with the 42-mer is apparently similar and may be it interacts with the major grove of the aptamer hence no significant structural rearrangement takes place. ${ }^{180}$ 


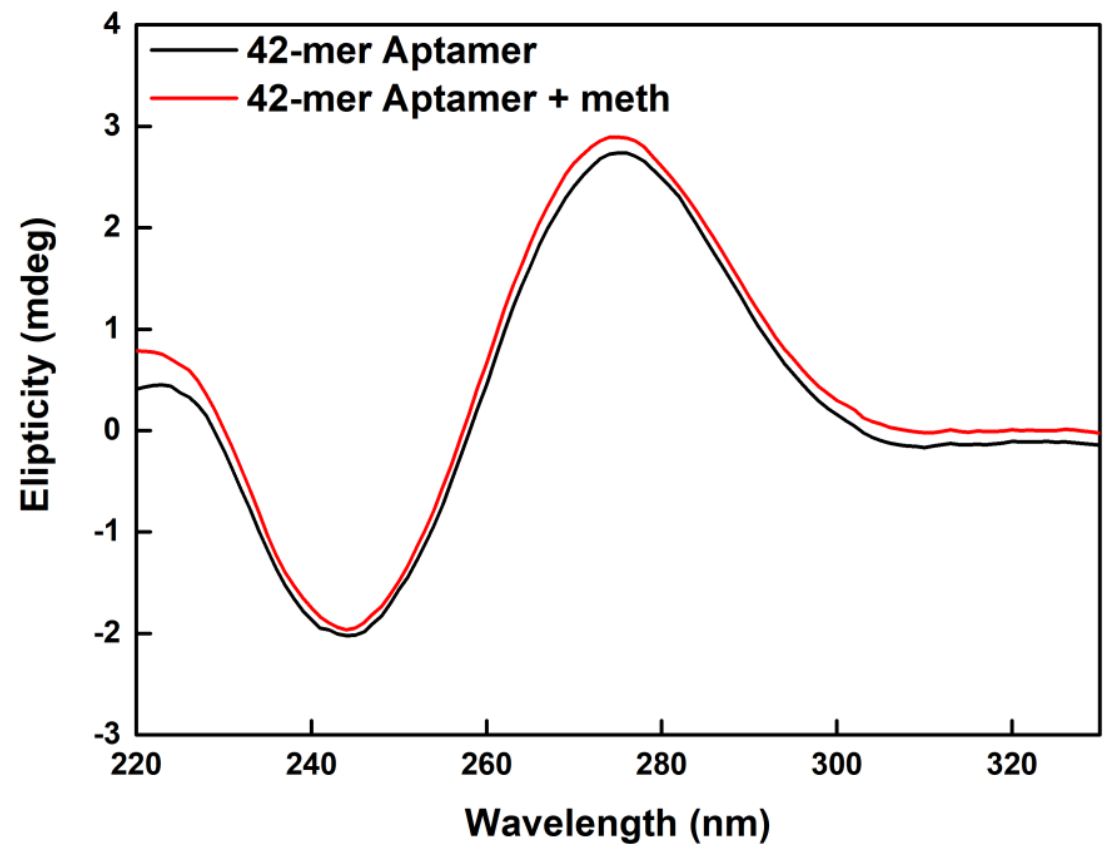

Figure 4.9 CD experimental data for 42-mer meth aptamer alone and in the presence of meth show the characteristic B-form ellipticity peaks at $274 \mathrm{~nm}$ (positive) and $244 \mathrm{~nm}$ (negative) suggesting no conformational change in the structure of aptamer induced by meth.

However, it may be predicted that the aptamer is more sensitive to the presence of meth in solution indicating stronger affinity which facilitates its desorption from the AuNPs to bind to target. Also, this could be owed to the effect of modified AuNP SELEX which accounted for the adsorptiondesorption kinetics and increased recognition sensitivity of non-immobilized meth in solution. 


\subsection{Chapter conclusion}

The findings in this chapter help support the proposed hypothesis and successfully addressed the problem discussed in the previous chapter (Chapter 3). It has been proved consistently, through comparisons, the effect of SELEX selection strategy on the sensor response. SELEX strategies modified to suit the downstream application of the aptamer in the corresponding sensing platform have positive outcomes compared to when they are not. It is also worth noting that the detection of small molecules comes with a lot of challenges especially when it comes to designing aptamers for immobilized small target molecules. This may lead to non-specificity as well as total loss of the aptamer's target identifying ability. In this chapter, a better performing aptamer selected by the AuNPs aggregation based SELEX was applied to the AuNPs aggregation assay sensing format to determine the effect of SELEX on the downstream performance of the aptamer and it was demonstrated that the modified SELEX strategy facilitated the development of aptamers with improved sensitivity and compatibility towards the target and assay format. Lastly, new generation 42-mer aptamer has shown a promising detection level of $10 \pm 3 \mathrm{nM}$ which is a 3 -fold improvement from the previously achieved LOD $(\sim 33 \pm 5 \mathrm{nM})$ with the 34-mer discussed in Chapter 3 . 


\section{CHAPTER 5}

\section{The nature of interferences when}

applying colorimetric aptasensors in human saliva 


\subsection{Abstract}

The detection of methamphetamine in oral fluid is a highly promising alternative diagnostic matrix compared to urine and serum. Detection of methamphetamine in oral fluid would pave way for the easy evaluation of impairment in drivers during roadside drug testing as well as ensure safe working environments by facilitating evaluation of impairment in employees at workplaces. In this chapter, the detection of meth in the challenging matrix of oral fluid has been discussed in detail. A membrane based point-of-care POC friendly pre-treatment technique has been developed which helped in the elimination of interferences caused by salivary proteins and facilitated the demonstration of meth detection in saliva using a AuNP based colorimetric aptasensor platform discussed previously in Chapter 3 and 4. It was found that the colorimetric response in saliva was always suppressed apparently owing to the matrix effects moreover the aptasensor platform was limited by its need for dilution which made it difficult to achieve detection at the cut-off concentrations in saliva. This chapter also describes the successful validation trials with saliva samples from healthy donors spiked with unknown concentrations of meth. The new generation SELEX selected aptamer was found to achieve better levels of detection compared to its 34-mer counterpart consistent with the idea discussed in chapter 4 about the impact of selection strategies on the performance of aptamers. Nevertheless, the detection of meth at nanomolar levels in saliva offers immense promise for the translation of these platforms for on-site diagnostic systems. 


\subsection{Introduction}

Saliva is a unique clear, hypotonic fluid which is composed of secretions from a variety of salivary glands. The term whole saliva is inclusive of secretions from both major and minor salivary glands. 90\% of the salivary secretions are from the submandibular, parotid, and sublingual glands (Figure 5.1). There are also innumerable minor salivary glands which contribute to about $10 \%$ in making up this complex fluid and they can be divided into labial, buccal, palatal, lingual and retromolar glands. ${ }^{193,16}$ The components of saliva include proteins including enzymes, antibodies, sugars, lipids, electrolytes etc. The various functions and composition of saliva can be found in Figure 5.3. The striated duct cells in salivary glands reabsorb ions such as sodium and chloride ions more than they secrete potassium and bicarbonate which makes saliva hypotonic with respect to plasma. ${ }^{194}$ In addition to salivary components whole saliva may also include several non-salivary components such as food debris, microorganisms, bronchial and nasal secretions, etc. which make makes it a unique body fluid.

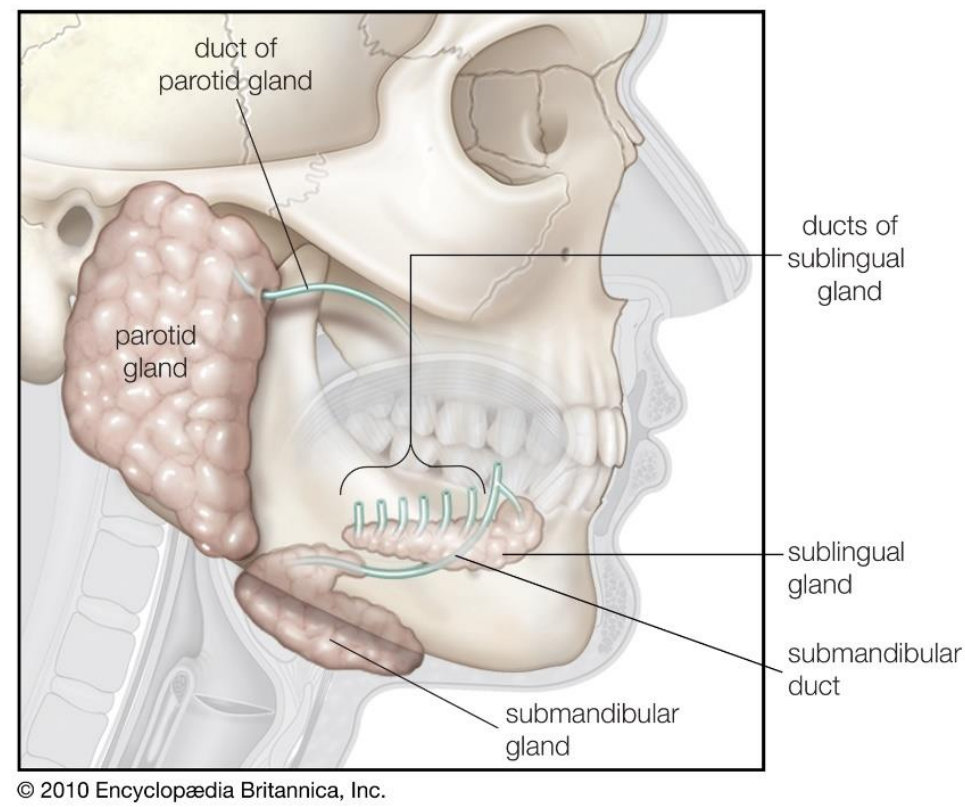

Figure 5.1 The three major pairs of salivary glands. Image taken from Encyclopedia Britannica.

Both intracellular and extracellular pathways facilitate the transport of biomolecules from blood to saliva. The major pathways of transport have been illustrated in Figure 5.2. Saliva is most often referred to as an ultra-filtrate of blood or more specifically it is the interstitial fluid which contains 
most analytes (biomarkers, drugs, hormones etc) at concentrations comparable to serum. ${ }^{16,195,196}$ The major mode of transport of drugs from blood plasma to saliva is via passive diffusion and the transport depends on numerous factors such as protein binding, pKa (hence charge), molecular weight of drugs, lipid solubility and stability in saliva. In order to reach saliva from plasma via diffusion, the molecule has to pass through 5 barriers: blood capillaries, interstitial space, cell membrane of the salivary cells or ducts, cytoplasm of the salivary cells and the luminal cell membrane. Drugs such as methamphetamine which are relatively small (<1900 Da), lipophilic and basic in nature with very low protein binding can easily pass through membranes via passive diffusion and through ultrafiltration because they are small enough to sneak in through the spaces between acinus and ductal cells. Protein bound drugs in the blood are inactivated and it is only the free form drug molecules that are physiologically active, and that free fraction is found in the saliva. The $\mathrm{pH}$ of saliva is slightly acidic ranging between 6.2 - 7.6 which facilitates the deposition of weakly basic drugs such as methamphetamine via ion trapping, in their unmetabolized form. ${ }^{196}$ This makes saliva an important subject for therapeutic and diagnostic applications. ${ }^{197,198,199}$ 


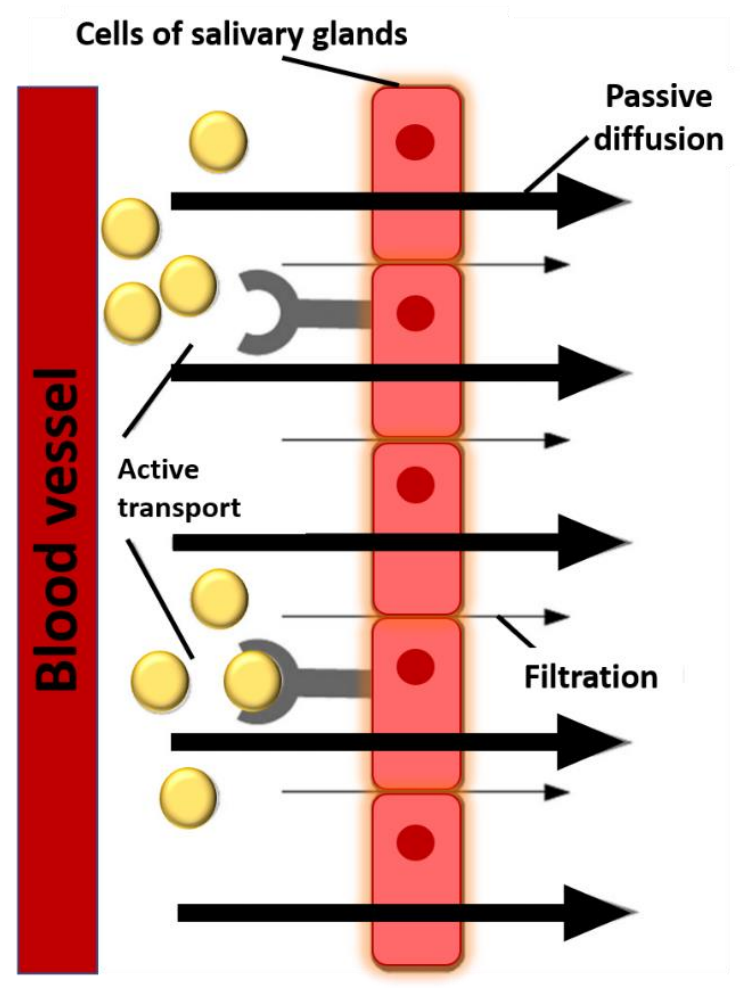

Figure 5.2 Illustration of major modes of transportation (passive diffusion, active transport, and ultrafiltration) of biomolecules and small molecules from blood to saliva. (The figure has been adapted and modified from Pfaffe et al. 2011)

Detection window of methamphetamine in oral fluid has been reported to be relatively shorter when compared to urine. ${ }^{199}$ Detection rate of methamphetamine in oral fluid has been moderate providing a positive test for up to 24 hours after cessation of use compared to urine which provided a detection rate lasting until 3 days as reported in a study. However, the concentration of oral fluid has been found to be higher in saliva when compared to plasma possibly due to the acidic nature of saliva as well as due to contamination of the mouth in cases where drug administration has been done orally. ${ }^{200}$ Both the detection window or the detection time and the rate of detection depend on several factors such as the sensitivity and specificity of the device, dosage pattern, analyte being detected etc. Overall, meth can be detected in oral fluid as early as $0.08 \mathrm{~h}-2 \mathrm{~h}$ following dosing providing a maximal detection of the drug within 2-12 hours. Oral fluid provides the earliest drug use indication.

Attention to using oral fluid in diagnostics is gaining a lot of momentum recently because of certain distinctive advantages it offers over other biofluids such as serum or urine which have been the 
traditional choice for drugs of abuse monitoring especially urine owing to the non-invasive sample collection it has to offer compared to venipuncture. However, urine sampling has been considered as involving an invasion to privacy because of the private act involved which requires privacy but at the same time provides the suspect with opportunities to adulterate the sample to evade accurate monitoring. This problem can be avoided with saliva as a diagnostic fluid as it's sampling can be performed with very little training and can be collected by the donor themselves without requiring any privacy (as in the case of urine collection) and prove cost-effective because of the nonrequirement of elaborate collection premises and procedures. Saliva collection is also suitable and widely applicable to any age group which enables screening of large population. Lastly, saliva drug concentrations indicate the actual unbound and free drug fraction or the circulating drug concentration which is an important indicator of impairment in individuals and was earlier regarded as an acceptable criterion for establishing impairment. Owing to the above mentioned reasons saliva may be considered as a promising alternative especially for drugs of abuse detection at roadside and workplaces. ${ }^{196,195,16}$

Application of saliva in AuNPs aggregation assays can be a challenging task owing to the very complex and variable matrix effect of the fluid. Moreover, the presence of proteins in saliva makes it even more problematic for applying to AuNPs which are known to be a well-established hotspot (owing to their high surface energy) for attracting proteins to their surface thereby forming protein corona which facilitates internalization of AuNPs in cells. ${ }^{201}$ For this reason, though AuNPs have been used extensively as carriers for therapeutic applications in biofluids, their application in conjunction with DNA aptamers as probes for drug detection in biofluids has never been reported before. For application in the biofluids as a sensor it's interaction with the omnipresent proteins in biofluids such as saliva in this case needs to be checked and kept to a minimum because that would lead to unwanted non-specific interaction or sensor fouling and would interfere with the specific response (coming from the interaction between aptamer and analyte of interest) of the biosensor that is being monitored. This problem mandates standardized collection, processing, or pretreatments before application to most biosensors.

Saliva sampling and processing technique plays a huge role in controlling the variability in sample constituents. Since saliva is a unique fluid and the composition and flowrate of saliva depends and varies not just amongst individuals but also within the same individual depending on the diurnal 
circadian, it is pertinent to be cautious about standardizing the collection and handling procedure (Figure 5.3). ${ }^{202,199}$ This is a very relevant problem faced when considering saliva as a diagnostic fluid and is probably the major reason for its less widespread applicability because when it comes to reproducibility and correlation between assay response involving saliva samples, it becomes a tricky situation. This calls for steps to standardize and process samples to limit these variations as much as possible without compromising the analyte concentration. ${ }^{203}$

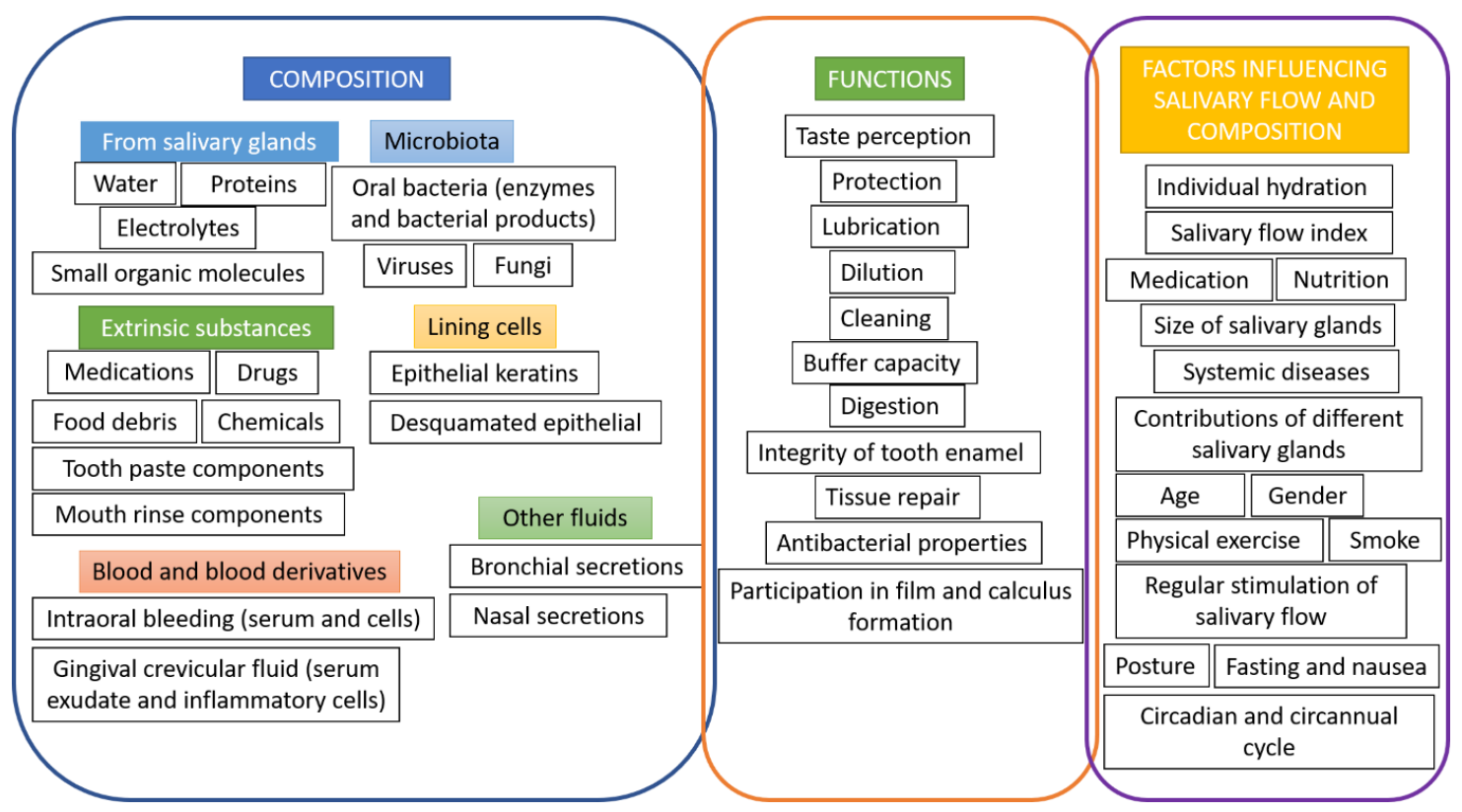

Figure 5.3 The image shows the composition, function and the factors affecting salivary flow and composition. This image has been inspired from the published work of Bellagambi et al. from the publication Saliva Sampling methods and devices.

In this work saliva pre-treatment procedures keeping in mind the POC friendly requirement have been developed and compared. It was found that a combination of syringe filters (which separates on the basis of pore size) and molecular weight (MW) cut-off filters (which separates on the basis of molecular weight) can give promising protein reduction levels in saliva samples without any extensive or laborious process involving overnight incubation etc. The overall protein level reduction was confirmed by DLS and UV-Vis. Subsequently, it was found that very promising levels of protein reduction in samples could be achieved by using a combination of PES membranes and Nylon membranes to remove most of the interfering proteins from samples without the need for a centrifugation step (which is required when using MW cut-off filters). The latter technique allowed the detection of meth at cut-off levels in oral fluid during the 
benchmarking and validation trials in collaboration with ESR. This led to the application of an AuNP aggregation assay aptasensors in saliva for the first time.

\subsection{Experimental section}

\section{Saliva collection}

Details on saliva samples collected by ESR: The saliva samples were collected using Quantasil collection devices, commonly used throughout Australia. They were collected from staff members of the Toxicology group of ESR. All staff members involved donated voluntarily and knew what the saliva was going to be used for, therefore ethics approval not required. All staff members who donated their saliva, were people who had not used drugs of any sort, including any type of prescription or over the counter medication. No staff members in the Toxicology group of ESR use methamphetamine

Saliva samples were sourced from about 12 different people. Depending on the stage of the project, different people were approached. For example, when testing for possible interference by common substances found in oral fluid, like caffeine or nicotine, someone who recently drank coffee or the one smoker in the group provided samples. When samples were being collected for spiking with the wide range of drugs tested, samples were taken well after any food or drink.

Method 1: Saliva was collected by passive drooling method wherein saliva was allowed to drain naturally without any forceful movement of the mouth, into a $10 \mathrm{~mL}$ plastic tube.

Method 2: QuantiSal collection kit was used to collect saliva.

\section{Pre-treatment of saliva samples}

Pre-treatment and subsequent use of saliva samples for target stock preparations were always carried out within 30 minutes of collection. It must be noted that the saliva processing techniques listed in the following section were tried and their performance was evaluated before moving to more targeted techniques of saliva processing. Figure 5.4 summarizes the different pre-treatment techniques tried and the successful ones have been highlighted with a green tick. 


\section{A) General physical pre-processing}

(i) Sonication and centrifugation: Sonication for 30 minutes followed by centrifugation (using Eppendorf Microcentrifuge Model 5424) at 10,000 rpm to remove any cell debris and solid precipitates. Sonication was done to denature and breakdown the large chain muco-polypeptide (mucins), a large chain mucopolysaccharides which impart the viscosity to saliva and make handling difficult.

(ii) Freeze-thaw centrifugation: The collected saliva sample was subjected to a freeze-thaw cycle before centrifuging at 10,000 rpm for 10 minutes. The freeze thawing was done to break down the mucins primarily and reduce the viscosity to facilitate sample handling. Centrifugation was done to remove cellular debris and other solid contents.

\section{B) Chemical pre-treatment to precipitate proteins}

Before the chemical treatments, collected saliva was subjected to centrifugation at 10,000 rpm to remove cellular debris and other solid particles.

(i) Acetone-TCA-DTT method: $500 \mu \mathrm{L}$ of pre-treated saliva $+500 \mu \mathrm{L}$ of $20 \%(\mathrm{w} / \mathrm{v}) \mathrm{TCA}-$ 90\% (v/v) acetone- $20 \mathrm{mM}$ DTT mixture and vortexed to mix thoroughly. The proteins were allowed to precipitate overnight at $-20^{\circ} \mathrm{C}$. Centrifugation at $15000 \mathrm{rpm}$ for 30 minutes and supernatant separated for further use.

C) Targeted pre-processing techniques- Membrane filtration based on molecular weight, pore size and charge

(i) 10 kDa centrifugation: Saliva samples were sonicated and centrifuged at 10,000 rpm before subjecting to Amicon ultra centrifugation through a $10 \mathrm{kDa}$ cut-off filter. The concentrate consisting of the proteins around $10 \mathrm{kDa}$ and higher were discarded and the ultrafiltrate was used for further analysis.

(ii) Combination of $10 \mathrm{kDa}$ centrifugal cut-off filter and $0.2 \mu \mathrm{m}$ GD/X-PES syringe filter: Collected saliva samples were passed through a $0.2 \mu \mathrm{m}$ graded density filtration system and Poly-ether sulfone membrane syringe filter (GDX-PES) to remove solid debris as well as large protein bodies which may be removed on the basis of size. This step allowed us to 
do away with the pre-processing steps of sonication and centrifugation. Then, it was passed through a $10 \mathrm{kDa}$ cut off filter in a microcentrifuge to remove proteins specifically.

\section{(iii) Sequential filtration through $0.2 \mu \mathrm{m}$ GD/X-PES followed by Nylon syringe filters:}

Collected saliva sample was directly diluted 4 times with buffer, then sequentially filtered through a $0.2 \mu \mathrm{m}$ GDX-PES membrane filter followed by a high protein binding nylon filter. The GDX-PES membrane filter could remove the solid contents of the sample as well as reduced the viscosity whereas the high protein binding nylon syringe filter could eliminate most of the proteins in the sample.

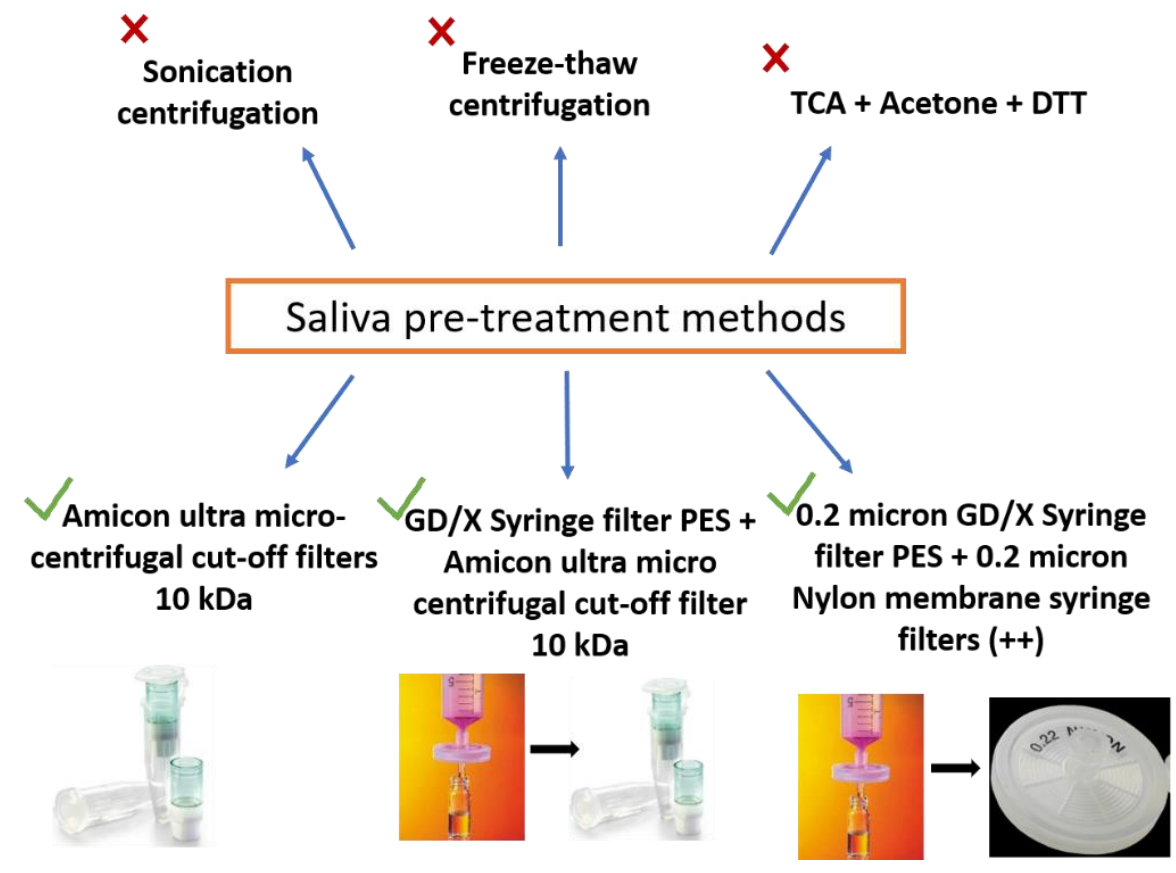

Figure 5.4 The different methods of saliva pre-treatment and processing to remove interfering proteins that were tried and tested. Red cross and green tick indicate the methods that were unsuccessful and successful to remove proteins respectively.

\section{UV-Vis characterization of AuNPs and saliva samples}

UV-Vis characterization was carried out to investigate the surface fouling of the AuNPs due to the salivary matrix after pre-treatment and with whole untreated saliva. Typically, $1.35 \mathrm{~mL}$ of AuNPs was mixed with $150 \mu \mathrm{L}$ of saliva such that the dilution of the saliva sample is 10 times.

\section{DLS investigation of saliva and AuNPs}


DLS investigations were mainly carried out to confirm and compare the protein reduction strategies with two promising techniques. $10 \mathrm{nM}$ AuNPs were used for DLS measurements. AuNPs + Aptamer + cut-off filter centrifuged saliva and AuNPs + Aptamer + syringe filtered saliva was compared using DLS. Since DLS alone cannot differentiate between aggregation and surface adsorption, UV-Vis has been used in conjunction to determine surface adsorption vs aggregation.

\section{Colorimetric aptasensing using conditioned/processed/pre-treated saliva sample to detect meth}

As described in Chapter 3, the same procedure for recording sensing response in oral fluid was carried out after spiking a conditioned or processed saliva sample with meth and quantifying the response using UV-Vis. The response obtained in saliva was also referenced against those obtained in buffer to account for the matrix effects. The saliva sample was also subjected to processing after adding meth to unprocessed and undiluted saliva to mimic the real-world scenario and the recovery of meth after filtration was estimated.

\section{Selectivity of the aptasensors}

In order to investigate the specificity of the aptasensor, the truncated 34-mer aptamer sequence was mixed with meth or other potential interferants (from the amphetamine class of drugs) including meth pre-cursor such as amphetamine, ephedrine, pseudoephedrine, MDA and MDMA, each at a concentration $200 \mathrm{nM}$. Selectivity studies involving a mixture of interferants were also conducted where each drug in the mixture had a concentration of at least $100 \mathrm{ng} / \mathrm{mL}$.

\subsection{Results and discussion}

\section{Evaluating the effect of whole saliva on AuNPs}

In order to evaluate the effect of saliva samples on AuNPs, undiluted whole saliva samples were added into AuNPs solution and it was observed that the solution remained red with no signs of aggregation even after subjecting the samples to $80 \mathrm{mM}$ ionic strength conditions (which was beyond the tolerable limit for even aptamer coated AuNPs). This observation suggested the effect of proteins which were imparting a strong resistance towards aggregation as illustrated in Figure 
5.5. It was found that whole saliva needed to be diluted 160 times (to eliminate effects of viscosity as well as overall matrix) to be applied to AuNPs sensor without any matrix interference (Figure 5.6 B). This was challenging because such high dilution was impractical considering the effect of dilution on the concentration of analyte.

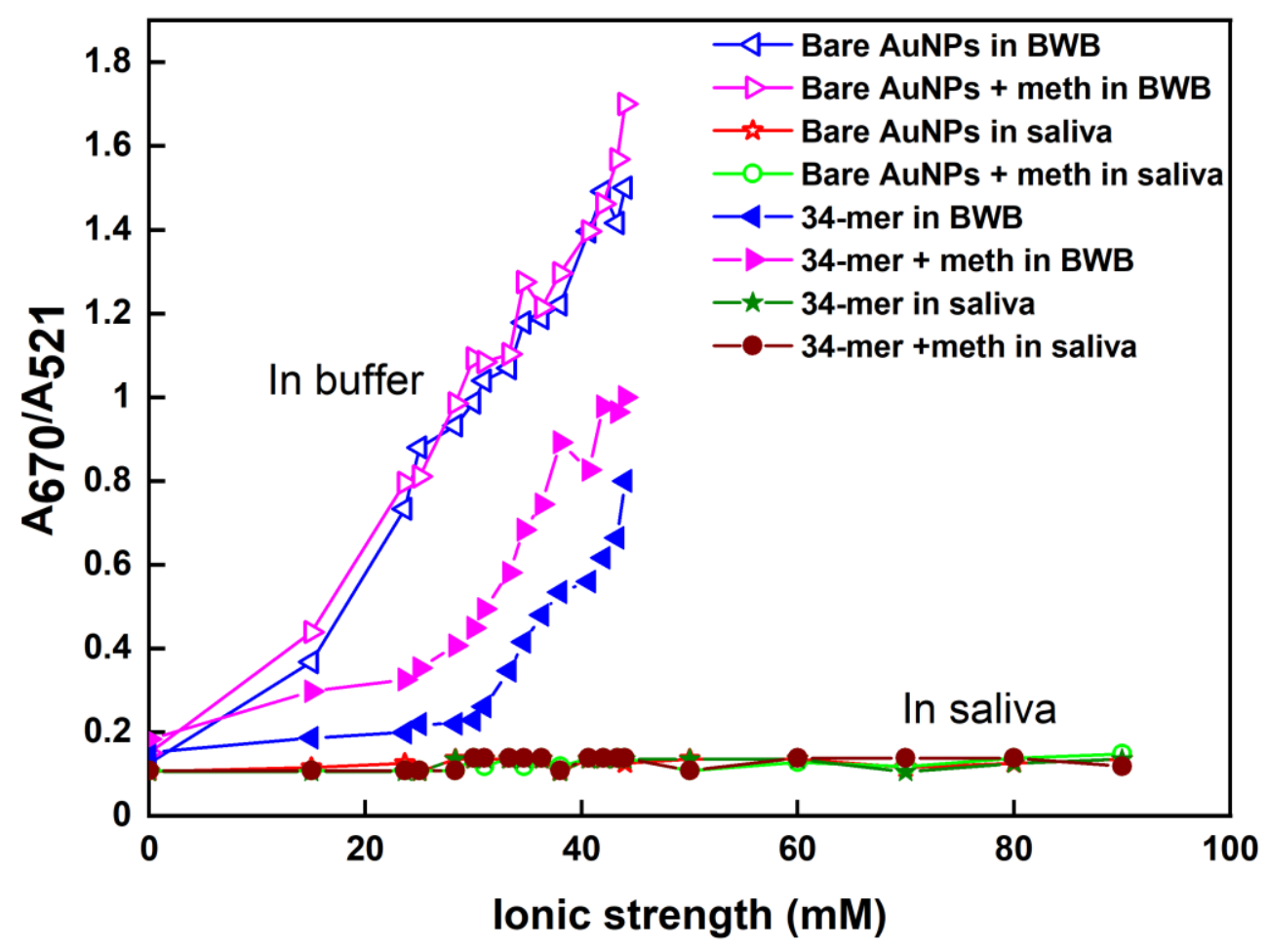

Figure 5.5 Graphical and visual illustration of the problem with application of undiluted/untreated whole saliva into the AuNP aggregation assay with and without aptamer protected AuNPs, clearly highlighting the interfering effect of the biomolecules in saliva which incapacitate the sensor completely. 


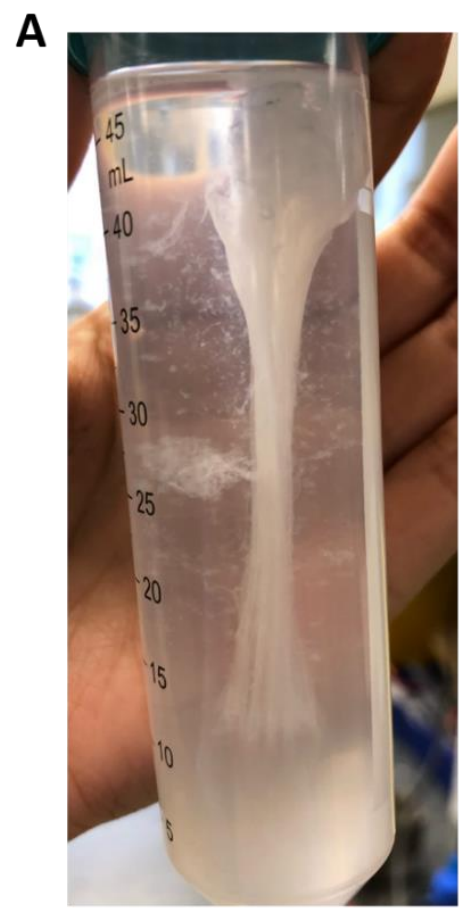

B

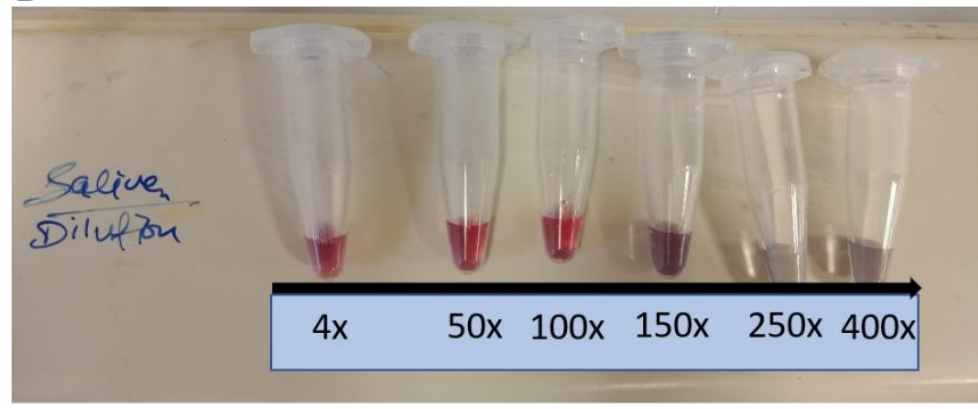

Figure 5.6 (A) Image of pre-treated saliva sample diluted with buffer after sonication for 30 minutes. The white clump could be a mixture of mucous, cell debris and food particulates. (B) Image showing that AuNPs could only be susceptible to aggregation to salt $(0.5 \mathrm{M} \mathrm{NaCl})$ when whole saliva was diluted more than 150 times.

When whole saliva was centrifuged/sonicated/filtered using syringe filter to remove the mucinous proteins (which decreased the viscosity to some extent), and added (undiluted) into bare AuNPs, it was found that within a few seconds the colour of the AuNPs solution changed from bright red to purple which suggested partial aggregation. It must be noted this aggregation was apparently not due to the ionic strength because previously it was observed that samples containing artificial saliva led to aggregation of aptamer coated AuNPs in the absence of target (when salt was added) in contrast to aptamer coated AuNPs in BWB which remained stable under the same ionic conditions (adjusted by adding appropriate amount of $\mathrm{NaCl}$ ). This suggested that the ionic strength was not the only variable that affected the AuNPs stability in saliva rather, the effect of the electrolyte composition i.e. specific interference from certain electrolytes in saliva may be the reason which compromised the sensor capability and gave false positives. ${ }^{204,121}$ Also, bare AuNPs in artificial saliva medium did not aggregate and remained stable suggesting the ionic strength of saliva or artificial saliva which is approximately $43 \mathrm{mM}$ (final concentration in sample $\sim 4 \mathrm{mM}$ ) 
was not enough to induce aggregation of AuNPs colloid (can tolerate upto $13 \mathrm{mM}$ ionic strength). However, in real saliva samples (without the mucins) bare AuNPs partially aggregated and so did aptamer coated AuNPs suggesting interfering reactions between bare/ aptamer coated AuNPs and the salivary matrix.
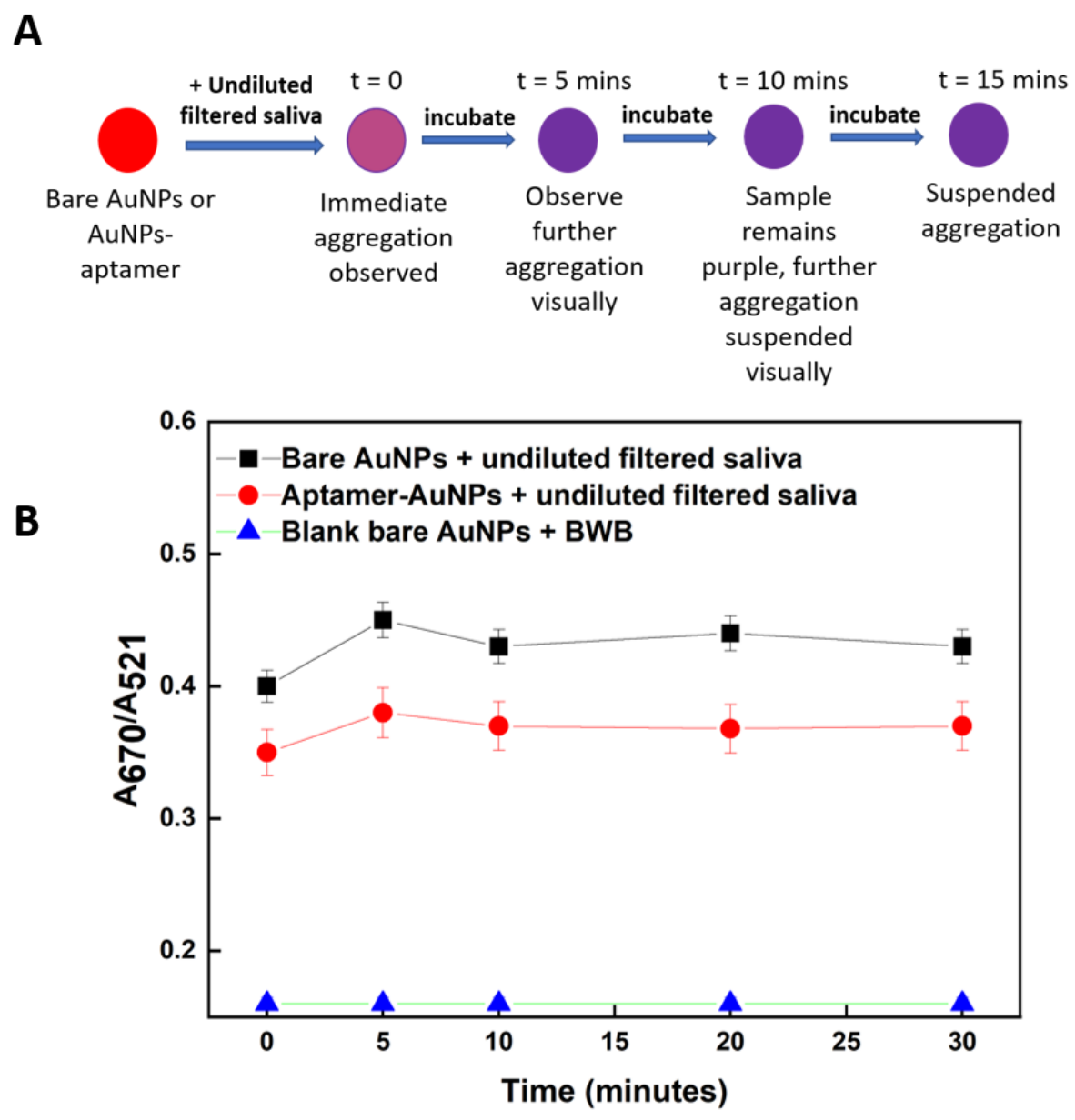

Figure 5.7 Kinetics of aggregation in real undiluted filtered (reduced mucins) saliva. The undiluted filtered saliva causes partial aggregation of bare as well as aptamer coated AuNPs immediately upon addition. However, after a few minutes the aggregation kinetics is stabilized due o the onset of protein adsorption and stabilization of AuNPs. Error bars represent the standard deviation of the mean of three measurements. 


\section{But why do the samples aggregate when filtered saliva is added?}

This question can be answered by considering that the decrease in the overall effect of mucinous proteins may have led to the effect of certain electrolytes or certain organic compounds to gain mobility/kinetic advantage over other proteins and react with AuNPs/AuNPs-aptamer to trigger aggregation immediately (which was not possible when the sample was too viscous as it might have impeded the movement of ions and components). Another possibility is that the interfering reaction between the AuNPs /AuNPs-aptamer and the electrolytes may have been happening but the presence of a blanket of long chain polypeptide might have inhibited the AuNPs to come in contact with each other (i.e. no plasmon coupling) and hence no red shift/visual colour change was observed as illustrated in Scheme 5.1 A which shows huddling of AuNPs together without plasmon coupling. In either case no colour change was visible due to the presence of mucoproteins/mucins. However, after the long chain polypeptide chains were reduced in content, the plasmon coupling effect may have been achieved and hence an immediate colour change is observed.

Therefore, it was hypothesized that the aggregation was induced by specific interaction between the electrolytes in filtered saliva (not ionic strength) and its effect of inducing aggregation was faster than the protein adsorption effect due to the kinetic advantage of those electrolytes which are able to reach the AuNPs surface faster than the other proteins in a reduced mucin environment i.e. filtered/sonicated/centrifuged saliva. In support of this hypothesis, it must be noted that in case of artificial saliva medium the aggregation triggered depicted a color change of the sample from red to purple to grey immediately indicating sedimentation at the end. In real saliva due to the presence of other on-mucinous proteins/biomolecules, the effect of the electrolytes in inducing aggregation was in competition with the opposite effect of other non-mucin protein stabilization and that is indicated by the purple color observed in the samples which remain purple over time without undergoing further aggregation/color change. This may be explained by the adsorption and stabilization of AuNPs by proteins which leads the sample to be in a partially aggregated stable state. The kinetics of aggregation is illustrated in Figure 5.7. This observation was important because it answers a key question regarding the contradicting effects of the salivary matrix on AuNPs stability. 


\section{Kinetics of electrolyte interference vs protein interference}

It was important to prove that both the kinetics of ionic strength and protein adsorption had opposing effects on the AuNPs sample but the final effect was a result of the overpowering effect of the protein stabilization kinetics which takes more time to come into effect compared to the electrolytes. However, the problem was undiluted saliva caused immediate aggregation which was in line with that observed with the interfering effects of artificial saliva and indicated that undiluted real saliva was unsuitable for application to AuNPs aggregation assay. Therefore, dilution was necessary for moving further.

By diluting saliva one time, it was found that the electrolyte interference effect could be eliminated as the samples did not aggregate immediately (as in case of undiluted saliva). But, the protein stabilization effect was still there and the samples were resistant to aggregation. However, this provided the opportunity to clearly prove the proposed hypothesis further regarding ionic strength having an immediate effect and protein adsorption happening after a certain incubation time and then leads.

2x diluted saliva (filtered/centrifuged/sonicated) did not aggregate the AuNPs sample as shown in Figure 5.8. Even after addition of $8 \mu \mathrm{L}$ high ionic strength $\mathrm{NaCl}(1 \mathrm{M})$ after an incubation period of 10 minutes the sample showed strong resistance to aggregation. To prove that this stabilizing effect happened due to proteins adsorption it was important to show that without an incubation time, there would be no stabilization effect. 


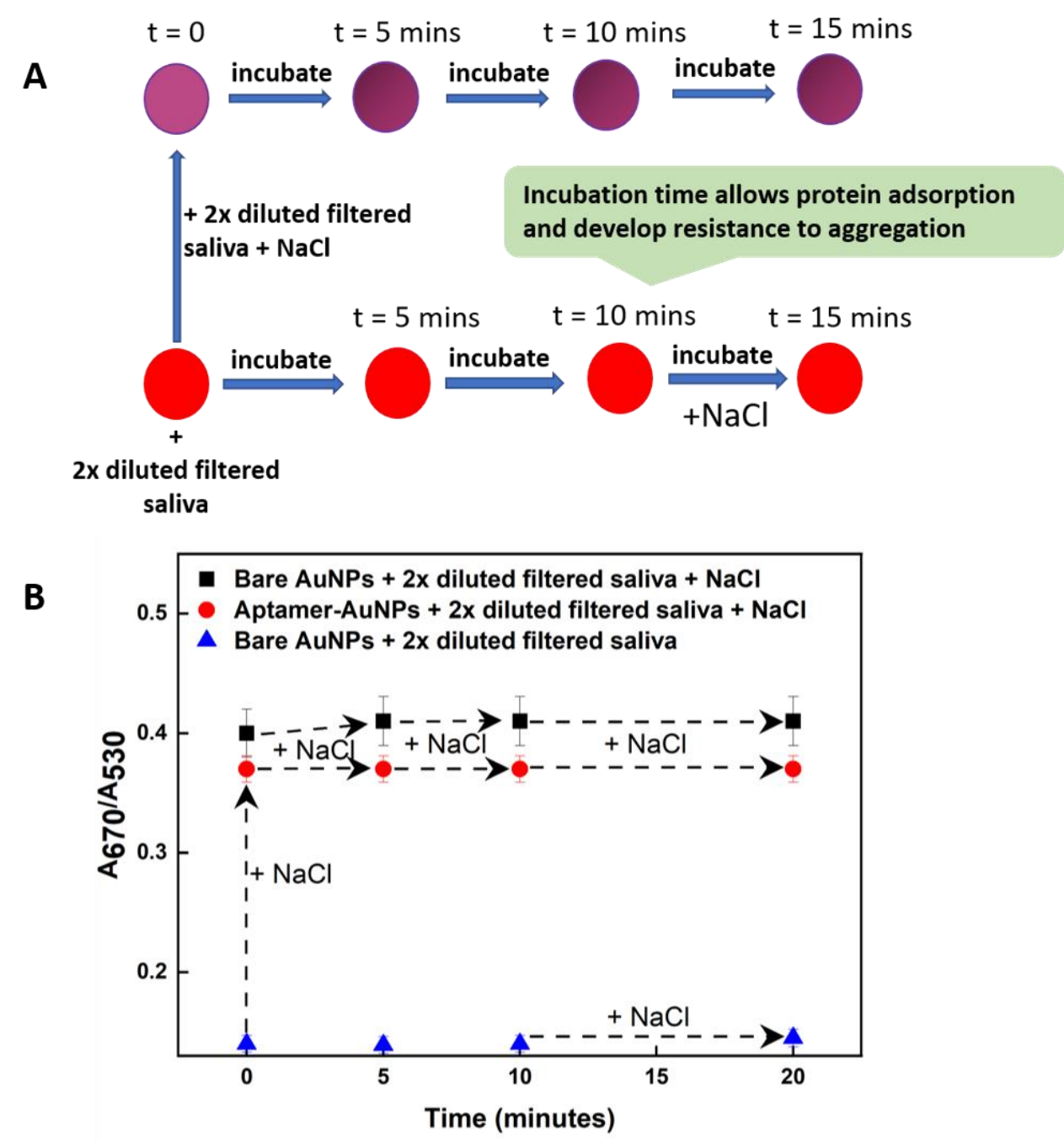

Figure 5.8 Kinetics of electrolyte interference vs protein interference. $2 x$ diluted filtered saliva (reduced mucins) shows a strong resistance to aggregation (blue points) after an incubation time of few minutes (510 minutes) which allows the proteins to adsorb on AuNPs and stabilize against salt induced aggregation. However, on the immediate addition of salt along with the $2 \mathrm{x}$ diluted filtered saliva, the particles (red and black points) partially aggregate due to ionic strength but after a few minutes, the aggregation kinetics stabilizes with the onset of protein stabilization effect such that further addition of salt fail to induce visible colour change. Error bars represent the standard deviation of the mean of three measurements.

Therefore, $2 \mathrm{x}$ diluted saliva was added and immediately $8 \mu \mathrm{L}$ high ionic strength $\mathrm{NaCl}(1 \mathrm{M})$ was also added and as per the hypothesis the sample was partially aggregated confirming the immediate effect of ionic strength due to the kinetic advantage of electrolytes over proteins. However, after 5 minutes more $\mathrm{NaCl}$ was added to see whether the partially aggregated state of the AuNPs can be further aggregated or not. However, it was found that on further addition of $\mathrm{NaCl}$ there was no 
aggravating the stability of the solution clearly proving that the protein adsorption effect needs time to stabilize the samples against salt induced aggregation. Having proved this, three clear conclusions overall were drawn:

1) whole undiluted/untreated saliva not suitable for application to AuNPs (as demonstrated in the beginning) due to viscosity due to mucinous proteins and their immediate stabilization effect Scheme 5.1 A.

2) Undiluted but filtered saliva (reduced mucins) were not suitable for application to AuNPs assay due to aggregation Scheme 5.1 B.

3) Filtered and $2 x$ diluted saliva was also not suitable for application to AuNPs due to protein stabilization effect and incubation was a necessary step in AuNPs aggregation assay Scheme 5.1 C.

\section{Therefore, further dilution was necessary to eliminate the protein effects but how much?}

Moving further, same protein stabilizing effect was observed for higher fold dilution (more than $4 \mathrm{x})$. The target was to achieve a dilution limit of $4 \mathrm{x}$ due to various reasons, first, $4 \mathrm{x}$ dilution was an acceptable dilution limit found in some conventional saliva collection devices and second, further dilution would only make it challenging to analyze ultra-low meth concentrations in diluted saliva. Therefore, a balance was necessary to mitigate the matrix effects while not diluting the sample too much.

Hence, it was imperative to process saliva samples to remove proteins before adding to AuNPs and then eliminate the $\mathrm{pH}$, viscosity and other matrix effects by dilution. Scheme $\mathbf{5 . 1}$ shows the illustrated possible mechanisms of saliva matrix interference with the AuNPs sensor all of which suggest the need for saliva processing measures for protein removal. 


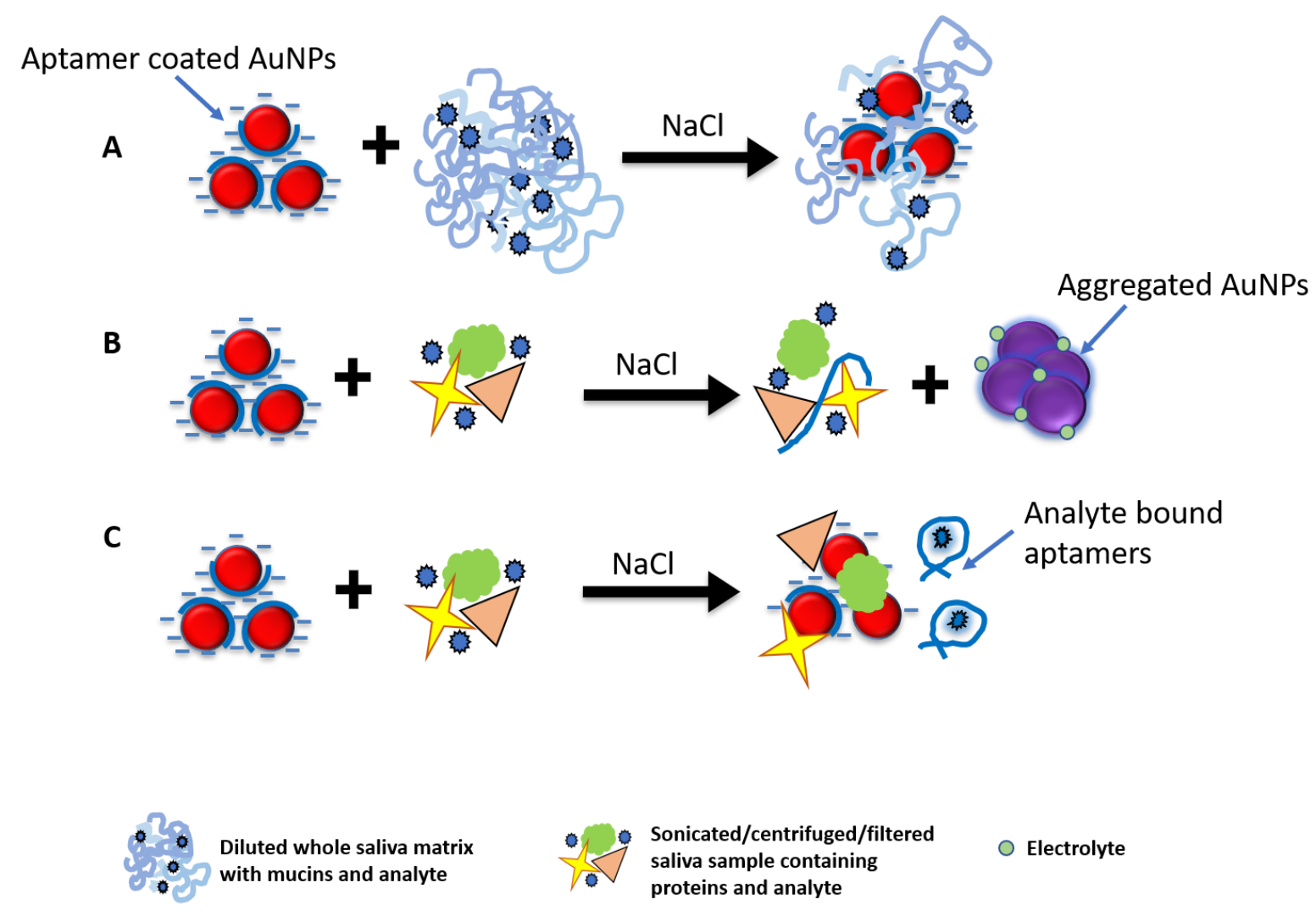

Scheme 5.1 Illustration of proposed mechanisms of interference from the salivary matrix and AuNPs aggregation aptasensors. A. Mucins/long chain polypeptides inhibit overall kinetics of target/aptamer movement by forming a viscous matrix which stabilized the AuNPs against aggregation hence the retention of red colour. B. sonicate/centrifuged/filtered saliva having reduced mucins may aggregate the AuNPs due to the electrolyte effect on AuNPs which may displace aptamers to interact with AuNPs leading to aggregation. C. Diluted saliva samples with reduced mucins eliminated the electrolyte effect but protein stabilization effect was strong due to which strong resistance to aggregation was observed.

\section{Protein elimination techniques and investigations}

\section{General physical processing and chemical precipitation techniques}

Physical processing techniques (freeze-thaw cycle-centrifugation and sonication-centrifugation), were unsuccessful in protein removal, they apparently only reduced the viscosity by removing mucins. In other words, the tolerance of AuNPs against salt induced aggregation was still very high indicating the dominance of proteins and other biomolecules in the samples after physical pre-processing. The total protein concentration or a detailed proteomic study was not investigated because it was neither the aim nor the requirement for this work. Anyway, after this observation it 
was realized that freeze-thaw cycles followed by centrifugation or, sonication followed by centrifugation were not effective in protein removal and therefore chemical protein extraction method was tried. A combination of acid (TCA), ketone (Acetone) and reducing agents (DTT) were used to denature and precipitate proteins. The mixture was left overnight followed by centrifugation to separate the precipitate. The supernatant was expected to be protein free to a certain extent. However, the presence of acid, alcohol and DTT was detrimental for use in the AuNPs assay. Subsequently, it became clear that chemical treatment to remove proteins was not a viable alternative owing to the dilution required afterwards to mitigate the $\mathrm{pH}$ and other effects of the chemically treated sample before application to AuNPs assay. Therefore, next steps involved more targeted pre-treatment methods.

\section{Targeted processing strategies}

Separation on the basis of molecular weight: Centrifugation at 10,000 $\mathrm{g}$ to remove the cell debris and other solid contents followed by collection and centrifugation of the supernatant with an Amicon ultra $10 \mathrm{kDa}$ centrifugal cut off filter to remove majority proteins on the basis of molecular weight (because most proteins in saliva are in the $20-40 \mathrm{kDa}$ range) was investigated. ${ }^{205}$ This technique showed a considerable reduction in the salt tolerance and also confirmed our hypothesis that the proteins were indeed interacting with the AuNPs and stabilizing them against salt induced aggregation.

However, in an effort to make the saliva processing steps POC friendly and short, it was found that a $0.2 \mu \mathrm{m}$ GDX/PES syringe filter could effectively remove solid contents from the saliva and also resulted in an overall reduction in the protein content followed by the $10 \mathrm{kDa}$ centrifugation for the final clear sample to be used in the sensor. ${ }^{203}$ 


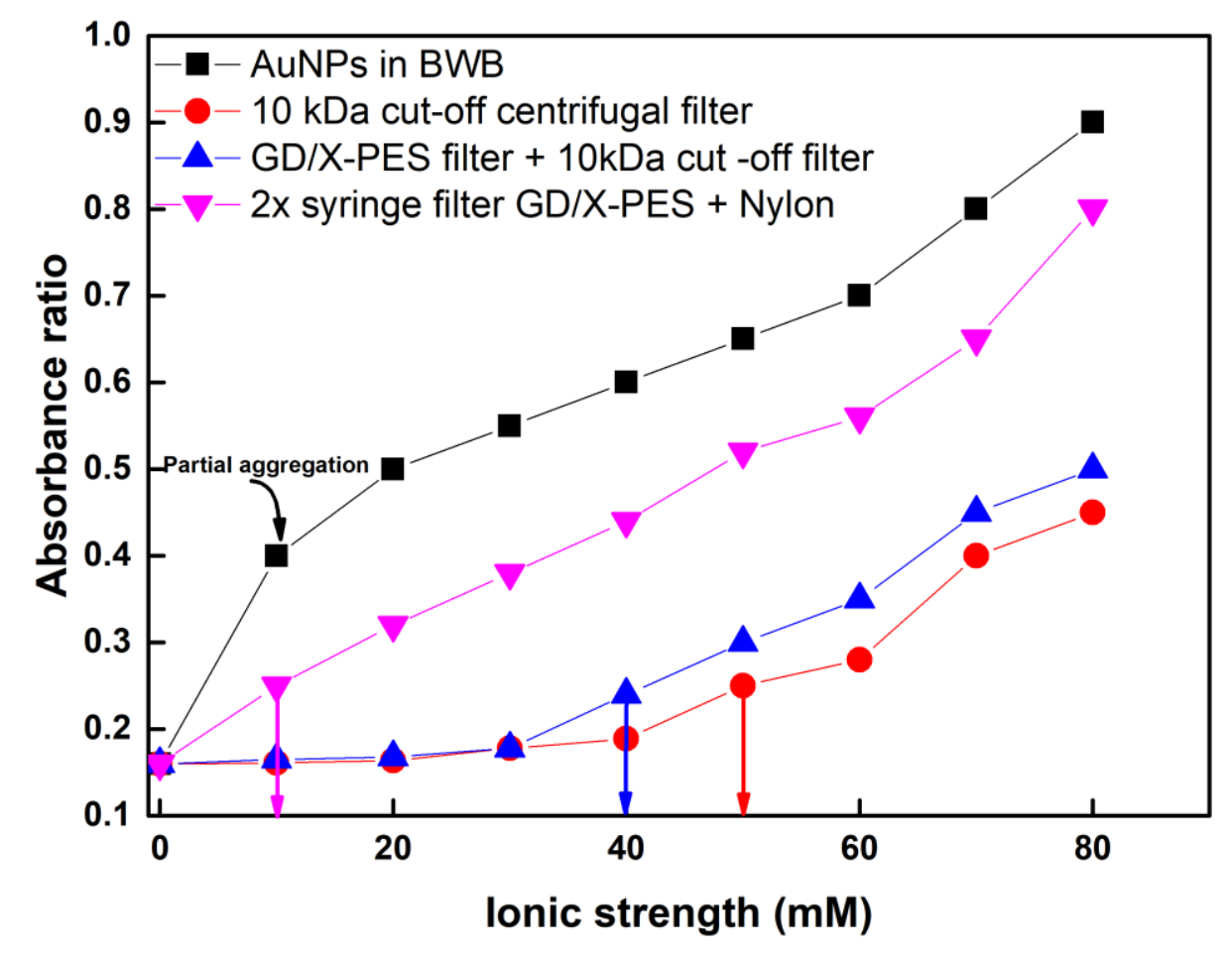

Figure 5.9 Graphical Illustration of the increasing sensitivity towards increasing ionic strength with each targeted protein removal strategy. 2x syringe filter strategy using PES and Nylon membrane filters were found to be most effective in protein removal.

Separation on the basis of charge: In further developments, it was possible to eliminate the centrifugation step completely by using a two-step syringe filter procedure wherein PES filters removed large mucopolysaccharides and solid contents and the high protein binding positively charged nylon membranes remove most negatively charged proteins from the samples on the basis of charge (because most proteins in saliva have a $\mathrm{pI}$ less than equal to 5 which indicates that they mostly exist in negatively charged form at $\mathrm{pH} 7.5) .{ }^{205}$

Therefore, among all the processing techniques these two proved most promising in reducing the protein content from the samples. Figure 5.9 illustrates graphically the improved sensitivity observed in a salt titration assay using the targeted protein removal strategies. 


\section{UV-Vis and DLS investigations to evaluate the extent of AuNPs surface fouling after targeted protein separation strategies}

The fact that the processing techniques (to remove proteins) involving $10 \mathrm{kDa}$ centrifugal cut-off

filter and a combination of syringe and centrifugal cut off filter were most promising was confirmed via UV-Vis and DLS measurements. The effect of filtered undiluted salivary matrix on the AuNPs using UV-Vis can be seen in Figure 5.10. Citrate stabilized bare AuNPs have a characteristic peak at $520 \mathrm{~nm}$ which undergoes partial aggregation depicted by an immediate colour change to blue/purple. The aggregation is indicated by a reduction in the plasmon peak intensity at $520 \mathrm{~nm}$ and a peak shift from $520 \mathrm{~nm}$ to a broader peak at $531 \mathrm{~nm}$.

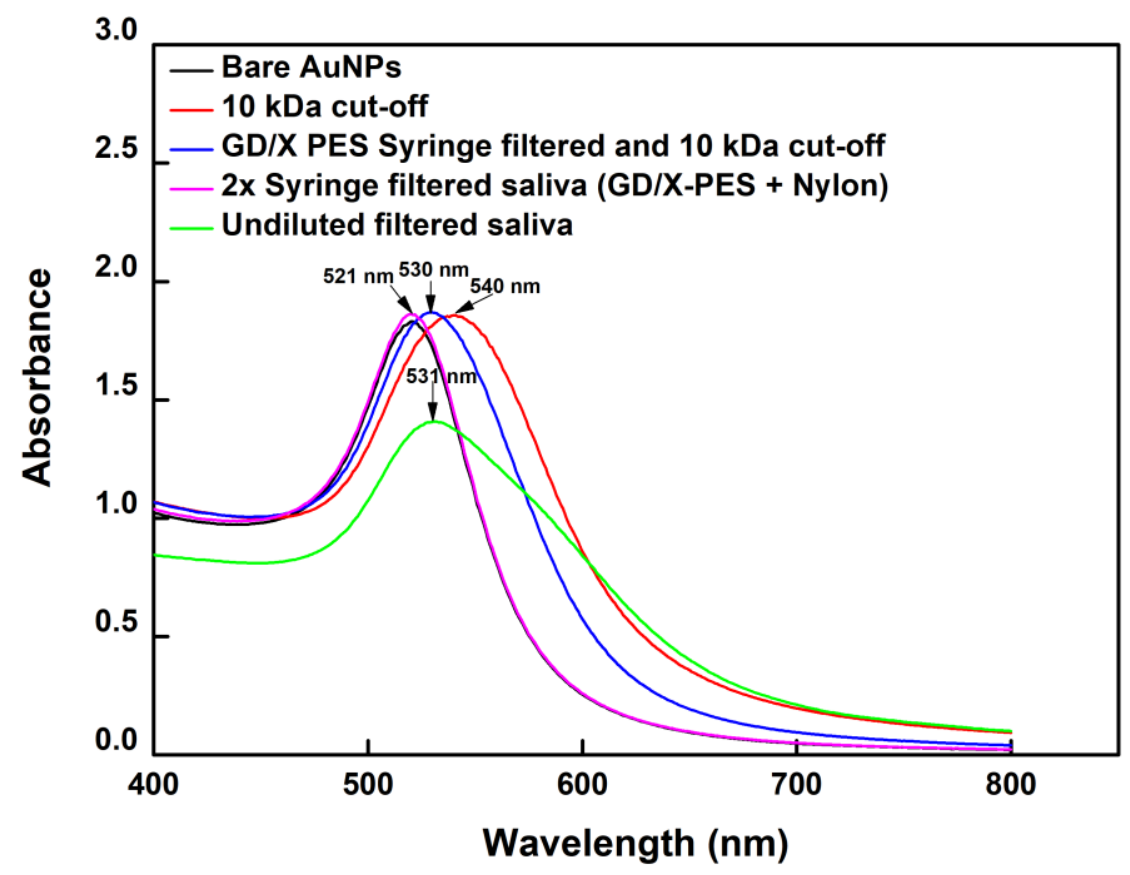

Figure 5.10 UV-Vis characterization of interactions between AuNPs and salivary matrix under different targeted processing strategies employed to pre-treat saliva.

Next, the effect of saliva sample subjected to centrifugation at 10,000g followed by centrifugation with a $10 \mathrm{kDa}$ cut-off filter (with $4 \mathrm{x}$ dilution) was added to the AuNPs + Aptamer sample and investigated. It was found that there was a significant surface fouling of the aptamer protected AuNPs. After exposure to saliva samples that had been processed with a $10 \mathrm{kDa}$ cut-off filter to 
remove proteins $10 \mathrm{kDa}$ and above, it was seen that there was a shift of the UV-Vis peak to 540 $\mathrm{nm}$ confirming the adsorption of proteins on the surface of AuNPs + aptamer samples but not aggregation (because the plasmon peak does not decrease in the intensity). Therefore the adsorption of proteins on AuNPs surface (indicated by a peak shift) suggests a change in the refractive index of the AuNPs. ${ }^{201}$ This may indicate two scenarios, one, the proteins adsorb to AuNPs at places not covered by the aptamers or the proteins are adsorbing to AuNPs by competitively displacing aptamers because AuNPs exposed to biological fluid have a high tendency to adsorb proteins in order to reduce their surface energy and form a stable (both tightly and loosely bound) protein corona. ${ }^{201}$

Using a GD/X-PES syringe filter followed by 10kDa cut-off filter led to a further decrease in the protein content indicated by a shift of plasmon peak to the left from $540 \mathrm{~nm}$ for the $10 \mathrm{kDa}$ sample to $529 \mathrm{~nm}$ which was consistent with the decreased resistance towards salt induced aggregation observed previously.

Finally, two step (2x) syringe filter-based separation was used to separate proteins and the resulting sample was added to the AuNPs sample. A $0.2 \mu \mathrm{m}$ GDX-PES membrane was used for reducing solid contents and cell debris and reduce the viscosity of the saliva sample and then filtered again through a high protein binding $0.2 \mu \mathrm{m}$ Nylon membrane to separate proteins from sample. Surprisingly, the UV-Vis data (Figure 5.10) showed and confirmed that the surface fouling is reduced considerably which is evident by a slight peak intensity change at $521 \mathrm{~nm}$. The plasmon intensity shift still indicated the presence of some biomolecules which adsorb to the AuNPs surface, however in comparison to other separation methods, this proved most promising due to the fact that it involved no sample processing downtime or use of laboratory specific instruments. 


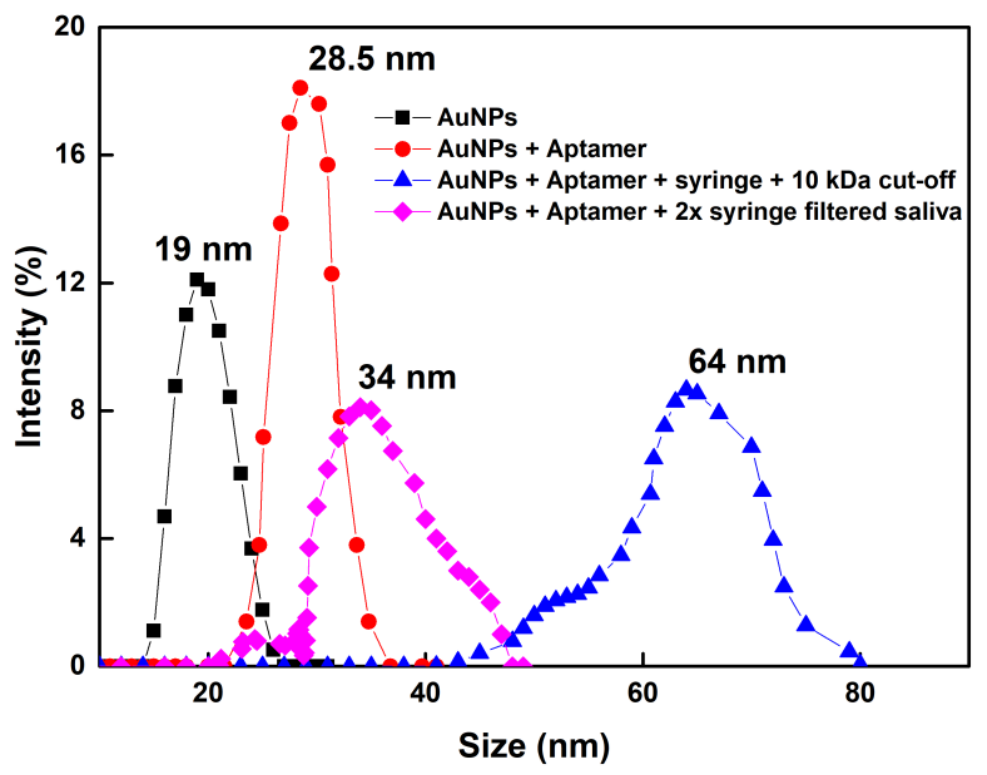

Figure 5.11 DLS characterization of the effect of two targeted protein removal approach on AuNPsaptamer samples. Among the two promising strategies, 2x syringe filter strategy for protein removal proved most efficient.

The protein adsorption to AuNPs was also confirmed via DLS as shown in Figure 5.11. It was found that the 2x syringe filter-based method enabled maximum protein removal compared to other targeted methods. It must be noted that DLS could not resolve between aptamer displacement from AuNPs surface by the salivary proteins or salivary protein interactions with aptamer coated AuNPs as shown in Figure 5.12. Moreover from the DLS data in Figure 5.12, bare AuNPs with the highly conditioned saliva (HCS) i.e. after treatment through PES and nylon syringe filters exhibited a size almost similar to that exhibited by aptamer coated AuNPs in HCS which showed that protein interaction with aptamer protected AuNPs was still possible and that the aptamers did not confer any protection from biomolecules (probably because of high affinity of proteins to AuNPs than aptamers which allowed them to displace aptamers from surface). Therefore, increasing aptamer coverage was not a solution.

In any case this knowledge would not have helped resolve the interference problem faced by the assay because it could be established that in either case, no visual response was obtainable, and 
the assay failed. Therefore, the primary aim was eliminating salivary proteins considerably to acceptable levels and that was successfully achieved.

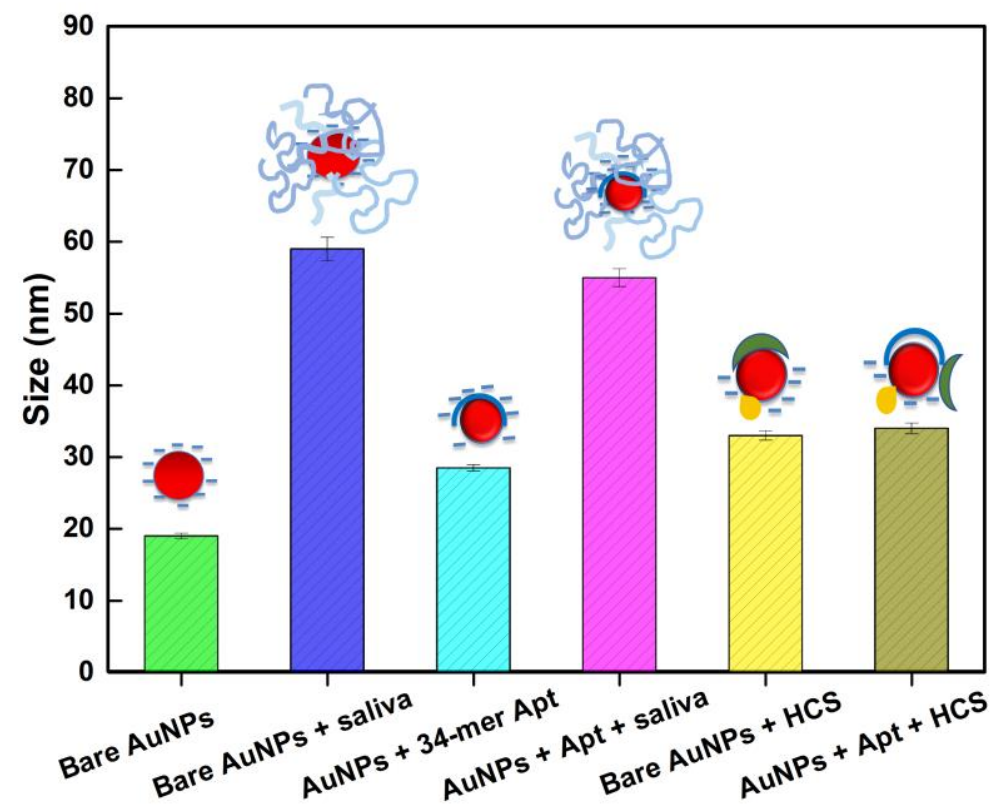

Figure 5.12 Summary of DLS size investigations of bare AuNPs with and without aptamer in diluted untreated saliva and in the final HCS (Highly conditioned saliva) obtained after GD/X syringe filter PES + Nylon syringe filter treatment.

\section{Validation of targeted protein removal strategies with random saliva samples}

Saliva samples from various healthy donors were collected using Quantisal collection kit and spiked with meth concentrations 800, 2000, 4000 and $8000 \mathrm{ng} / \mathrm{mL}$. These samples were provided by Helen Poulsen from ESR as part of a validation trial to test the validity of our saliva processing technique and sensing assay developed.

The spiked saliva samples obtained were received in a Quantisal collection tube (diluted 4 times with buffer). These saliva samples containing meth were pre-treated in the lab initially using the GD/X PES syringe filter followed by $10 \mathrm{kDa}$ centrifugal cut-off filter. It was observed that the concentrations over $4000 \mathrm{ng} / \mathrm{mL}$ ( $2680 \mathrm{nM}$ final concentration in assay sample) were likely to be detected by the assay with greater reproducibility than those below $4000 \mathrm{ng} / \mathrm{mL}$. 4 out of the 5 trials conducted were tested using the syringe filter combined with centrifugation method stated 
above. In the $5^{\text {th }}$ trial, the $2 \mathrm{x}$ filtration technique with GD/X 0.2 micron PES syringe filter and nylon membranes was used (as it was developed eventually) and it was found that the POC friendly procedure was very efficient, a finding consistent with the findings till now. Only in the $5^{\text {th }}$ trial concentrations $800 \mathrm{ng} / \mathrm{mL}(\sim 530 \mathrm{nM})$ could be detected easily. These trials with random saliva samples helped to validate the pre-treatment strategies developed.

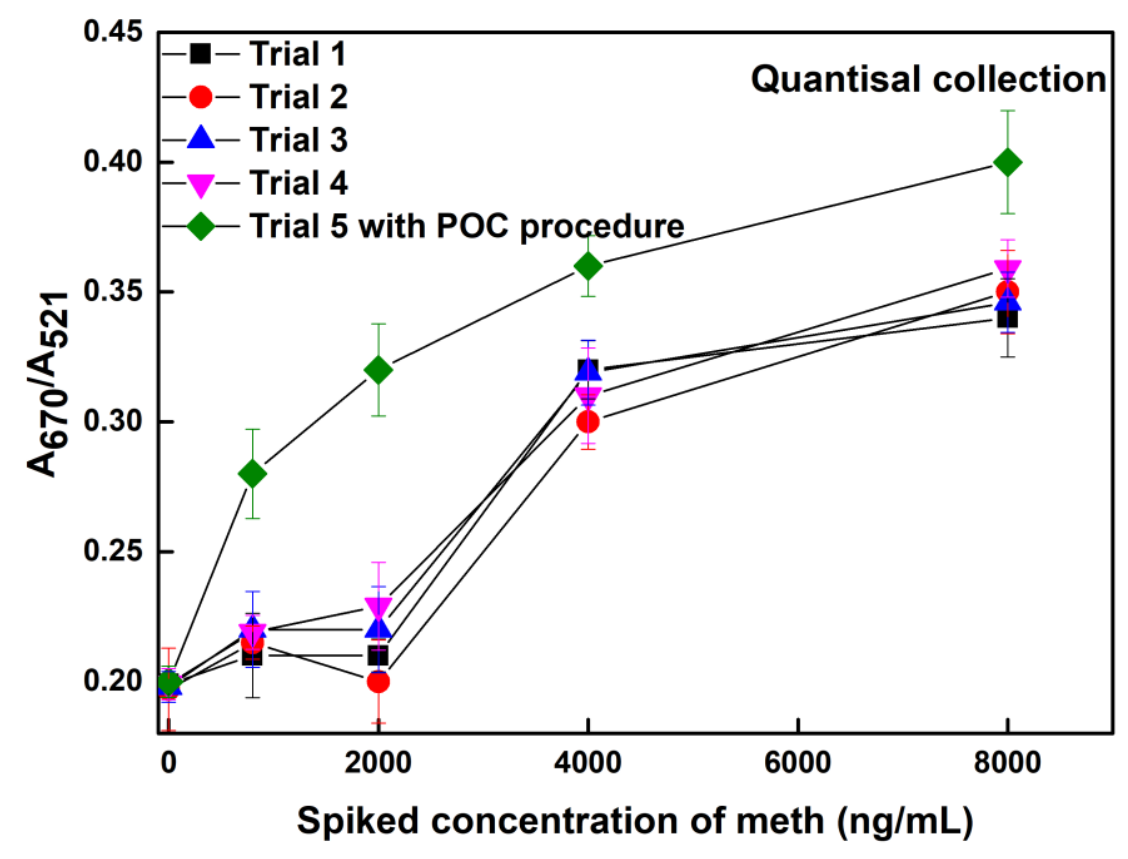

Figure 5.13 Plot clearly showing the improvement in response obtained by using the POC technique (sequential syringe filtration through $0.2 \mu \mathrm{m}$ GD/X PES membrane and $0.2 \mu \mathrm{m}$ positively charged Nylon membrane) to remove proteins. All data shown with the 34-mer aptamer. Error bars represent standard deviation of the mean of three measurements.

The validation findings were very promising because it confirmed the hypothesis that the positively charged nylon membranes were further able to remove majority of the proteins on the basis of charge.

\section{Calibration of response obtained with the 34-mer in saliva using association assay}

Figure 5.14 shows the optimization data for the 34-mer aptamer in oral fluid. The optimal ionic strength was found to be $53.6 \mathrm{mM}$ - indicating a high resistance to aggregation, presumably due 
to the proteins and other biomolecules in the complex salivary matrix blocking the surface of bare as well as aptamer coated AuNPs.

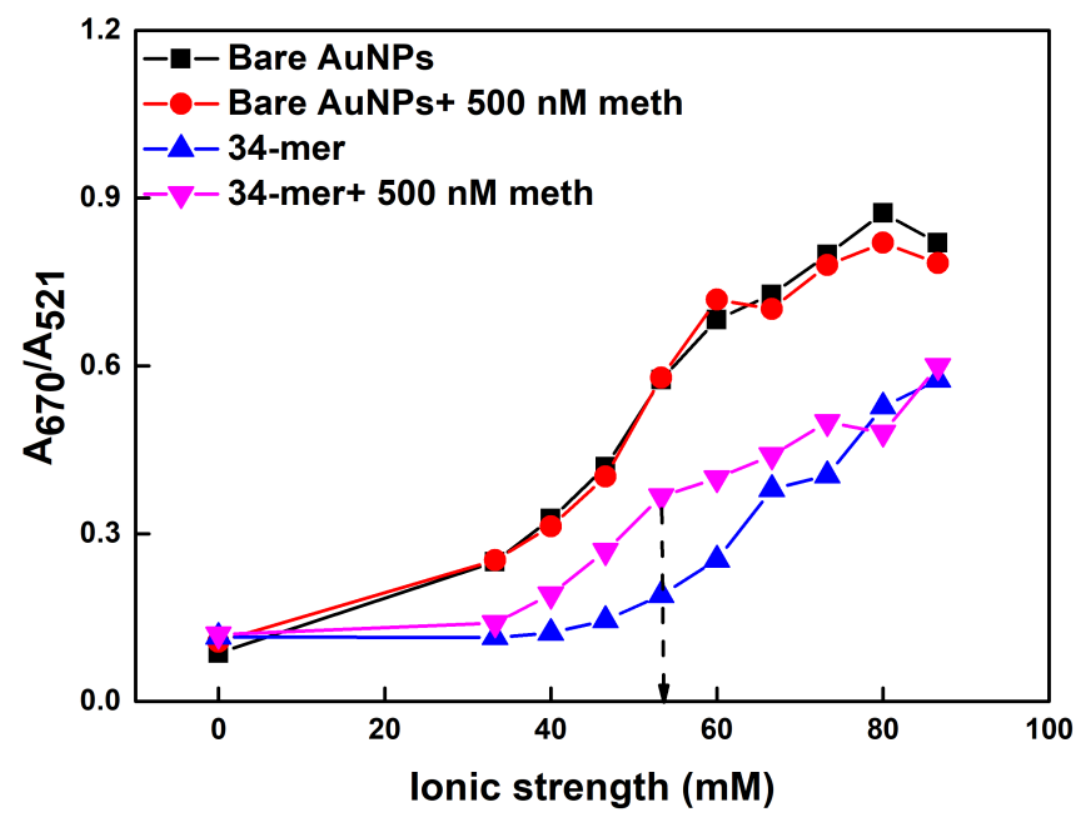

Figure 5.14 Salt optimization of 34-mer aptamer in oral fluid. The optimal salt concentration was found to be $53.6 \mathrm{mM}$ indicated by the arrow.

The sensing response in Figure 5.15 shows a linear range from 50 to $500 \mathrm{nM}\left(\mathrm{R}^{2}=0.90\right)$. The calculated LOD was found to be $161 \mathrm{nM}$ in diluted saliva medium (or $644 \mathrm{nM}$ in undiluted saliva medium). These signals were referenced against the standard calibration curve $\boldsymbol{y}=\mathbf{8 . 0 4} \times \mathbf{1 0}^{-4} \boldsymbol{x}+$ 0.23308 obtained in buffer for the association assay (Chapter 3) to quantify the effect of matrix interference on the sensor response as seen in Table 5.1. It was found that the detection of meth in saliva was always less than that in buffer - on average suppressed by approximately $47 \%$ at biologically relevant meth concentrations. It should be noted that the saliva collection method used here was passive drooling. This signal suppression is likely due to salivary proteins sequestering meth or interfering with the colorimetric response of AuNP-aptamer system (e.g., blocking meth binding or AuNP aggregation), or perhaps both. It was noted that the saliva collection method needed to be highly standardized for obtaining a reliable assay response. When collecting saliva on any other time of the day and under any other condition than that reported in this work resulted in failure of obtaining an assay response. Despite these practical application challenges, the 
improved sensitivity gained from the understanding of AuNP-aptamer interactions led to the first demonstration of a colorimetric aptasensor for meth in oral fluid.

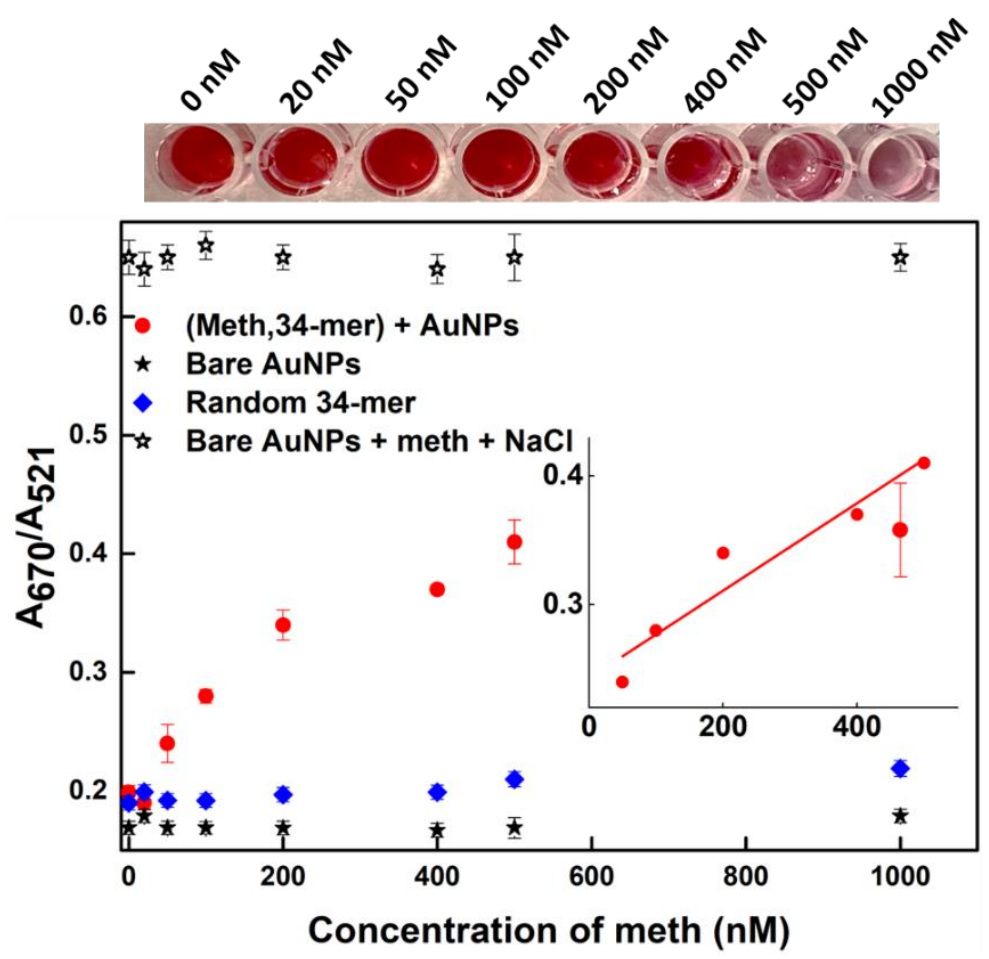

Figure 5.15 Colorimetric response of the 34-mer aptamer using the association assay in oral fluid with bare AuNPs and random-34 mer controls. Inset showing the linear fit. Error bars represent standard deviation of the mean of three measurements.

Table 5.1 The measurement of meth detected in oral fluid.

\begin{tabular}{|l|l|l|l|l|l|}
\hline $\begin{array}{l}\text { A670/A521 } \\
\text { ratio }\end{array}$ & $\begin{array}{l}\text { Spiked } \\
\text { concentration } \\
\text { of meth }(\mathrm{nM})\end{array}$ & $\begin{array}{l}\text { Concentration } \\
\text { of meth in } \\
\text { assay }(\mathrm{nM})\end{array}$ & $\begin{array}{l}\text { Found meth } \\
(\mathrm{nM})\end{array}$ & Recovery (\%) & RSD (\%) \\
\hline 0.248 & 500 & 50 & 18.5 & 37 & 2 \\
\hline 0.280 & 1000 & 100 & 57.4 & 57.4 & 4.3 \\
\hline 0.340 & 2000 & 200 & 132.89 & 66.4 & 6.1 \\
\hline
\end{tabular}




\section{Estimating loss of meth due to syringe filtration}

The evaluation of the loss of meth due to syringe filtration was also investigated as shown in Figure 5.16 and it was found that on an average, 90\% of meth can be recovered after filtration. This could be owed to the small size of the meth and some loss depicts the meth lost due to sequestration by the proteins. The recovery rates indicate the success of implementing the AuNP aggregation assay platform in detecting meth in treated whole saliva.

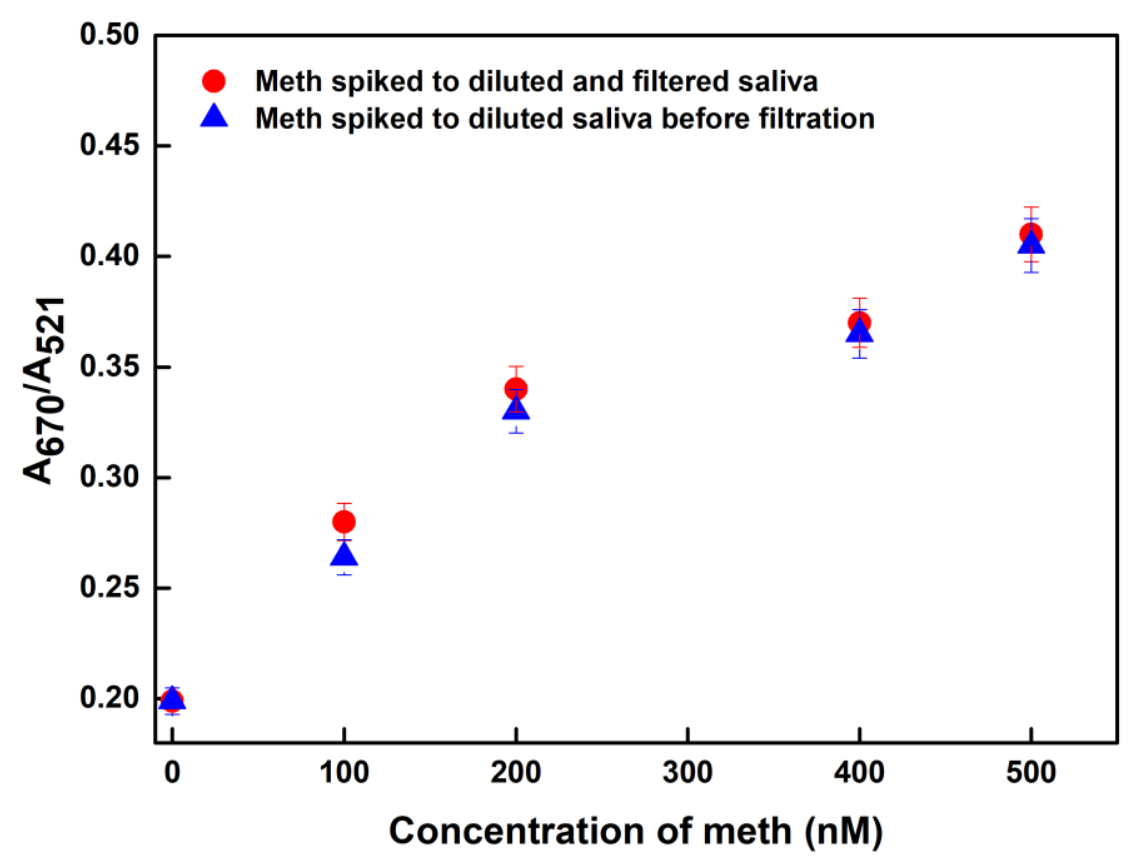

Figure 5.16 Graphical illustration of the effect of filtration on the concentration of meth. It was found that on an average more than $90 \%$ of meth could be recovered after filtration. Error bars represent standard deviation of the mean of three measurements.

\section{Estimating recovery of meth when meth is spiked into undiluted saliva}

The recovery of meth after spiking meth in whole oral fluid prior to pre-treating the sample and adding to the sensor (as would be the case in real scenarios) was investigated to estimate the loss due to pre-treatment of oral fluid as shown in Table 5.2. The concentrations spiked were 1000, 3000 and $5000 \mathrm{nM}$. The signal obtained was referenced against the calibration curve obtained in 
saliva $y=3.4 \times 10^{-4} x+\mathbf{0 . 2 4 3}$ to estimate the predicted concentration of meth spiked in undiluted saliva.

Table 5.2 The spiked vs predicted concentrations obtained when meth was spiked before dilution and pretreatment to evaluate real world application suitability. Concentration in undiluted saliva is obtained by multiplying by 10 (to account for assay dilution) and multiplying by 4 (to account for buffer dilution of saliva)

\begin{tabular}{|l|l|l|l|}
\hline $\mathrm{A}_{670} / \mathrm{A}_{521}$ & $\begin{array}{l}\text { Spiked concentrations in } \\
\text { undiluted saliva }(\mu \mathrm{M})\end{array}$ & $\begin{array}{l}\text { Actual predicted } \\
\text { concentration in } \\
\text { undiluted saliva }(\mu \mathrm{M})\end{array}$ & RSD $(\%)$ \\
\hline 0.246 & 1 & 0.8 & 2.6 \\
\hline 0.270 & 3 & 3.1 & 4.1 \\
\hline 0.30 & 5 & 6.7 & 3.4 \\
\hline
\end{tabular}

\section{Validation of the assay response with unknown saliva samples using dissociation assay}

Five saliva samples with unknown concentrations of meth spiked were provided. A blank for optimization purposes was provided as well. The saliva samples X1, X2, X3, X4 and a blank X0 were tested using the dissociation assay. The A670/A521 response values obtained were compared to the reference saliva sample calibration curve obtained via the dissociation assay to obtain a predicted value.

It was found that the predicted values obtained from calculation using the linear equation $\boldsymbol{y}=\mathbf{1 . 6}$ $\times \mathbf{1 0}^{-4} \boldsymbol{x}+\mathbf{0 . 2 1 7 3 2}$ for the saliva calibration curve were $342.6,550.5,847.6$ and $1085.2 \mathrm{nM}$ (final concentrations in assay). The actual spiked concentrations were then obtained from ESR and they were, 0, $800(5360 \mathrm{nM}), 2000(13402 \mathrm{nM}), 4000(26804 \mathrm{nM})$ and $8000(53608 \mathrm{nM}) \mathrm{ng} / \mathrm{mL}$ as obtained and assuming that the loss of meth during filtration is not too significant, the final concentrations (considering the dilution factor is 10 in the assay) in the AuNP assay sample may 
be assumed to be approximately $80(536 \mathrm{nM}), 200(1340 \mathrm{nM}), 400(2680 \mathrm{nM})$ and $800 \mathrm{ng} / \mathrm{mL}$ (5360 nM).

Table 5.3 Spiked vs predicted values when referencing against passive drooling collection method of saliva. Concentration in undiluted saliva is obtained by multiplying by 10 (to account for assay dilution) and multiplying by 4 (to account for buffer dilution of saliva)

\begin{tabular}{|l|l|l|}
\hline $\mathrm{A}_{670} / \mathrm{A}_{521}$ & $\begin{array}{l}\text { Spiked unknown concentrations } \\
\text { received }(\mu \mathrm{M})\end{array}$ & $\begin{array}{l}\text { Actual predicted concentration in } \\
\text { undiluted saliva }(\mu \mathrm{M})\end{array}$ \\
\hline 0.28 & 5.36 & 13.68 \\
\hline 0.31 & 13.4 & 22.02 \\
\hline 0.36 & 26.8 & 33.90 \\
\hline 0.4 & 53.6 & 43.40 \\
\hline
\end{tabular}

It is evident that the predicted concentrations using the calibration equation from the reference saliva samples (collected using passive drooling) are greater than the actual concentrations spiked. This was expected because the collection methods of the unknown samples were different from the collection method used when making the saliva calibration curve. Therefore, this actually highlights the effect of collection procedure on the sensor response. A possible explanation is that the saliva samples collected via the Quantisal procedure had less effect of the salivary matrix to begin with owing to the design of the saliva collection kit which has a cellulose pad that absorbs $1 \mathrm{~mL}$ of pooled saliva in the mouth before placing it back into a tube containing $3 \mathrm{~mL}$ buffer. Ideally this device is supposed to facilitate the extraction of the drug from the cellulose pad into the buffer with minimum salivary contamination and the buffer containing drugs are used for subsequent analysis. However, in the present scenario, this device has been primarily used to standardize collection procedure and obviously, since saliva was collected from healthy and nonmeth users, meth was subsequently spiked into the tube containing buffer (after accounting for the dilution factor) after incubating the cellulose pad containing saliva for a few hours. This may have resulted in a sample with little salivary interference compared to the method used in the laboratory which deals with results in more salivary interference. This explains the stronger signal. 
Therefore, it was hypothesized that the predicted values would have almost matched with the unknown values had the reference calibration curve used to predict been in saliva samples collected via Quantisal. Therefore, a calibration curve in saliva collected using Quantisal collection method was used to obtain a standard calibration curve. Using the equation of the curve $\boldsymbol{y}=\mathbf{1 . 8} \times \mathbf{1 0}^{-4} \boldsymbol{x}+$ 0.23893, the predicted values of the unknown samples can be found in Table 5.4. The values obtained were still offset but it was nearer to the actual values than those obtained when compared against a different collection method. Figure 5.17 shows the relationship between spiked and predicted values using the sensor assay. The predicted values were found to be correlatable with the actual spiked values with some discrepancies because of manual error and as well as due to effects of sample variability. Despite the challenges the validation trials were promising. However, more validation trials could not be done and may be part of future plans especially using the association assay.

Table 5.4 Spiked vs predicted values when referencing against Quantisal collection method of saliva. Concentration in undiluted saliva is obtained by multiplying by 10 (to account for assay dilution) and multiplying by 4 (to account for buffer dilution of saliva)

\begin{tabular}{|l|l|l|}
\hline $\mathrm{A}_{670} / \mathrm{A}_{521}$ & $\begin{array}{l}\text { Spiked unknown concentrations } \\
\text { received }(\mu \mathrm{M})\end{array}$ & $\begin{array}{l}\text { Actual predicted concentration in } \\
\text { undiluted saliva }(\mu \mathrm{M})\end{array}$ \\
\hline 0.28 & 5.36 & 7.89 \\
\hline 0.31 & 13.4 & 15.56 \\
\hline 0.36 & 26.8 & 26.51 \\
\hline 0.4 & 53.6 & 36.28 \\
\hline
\end{tabular}




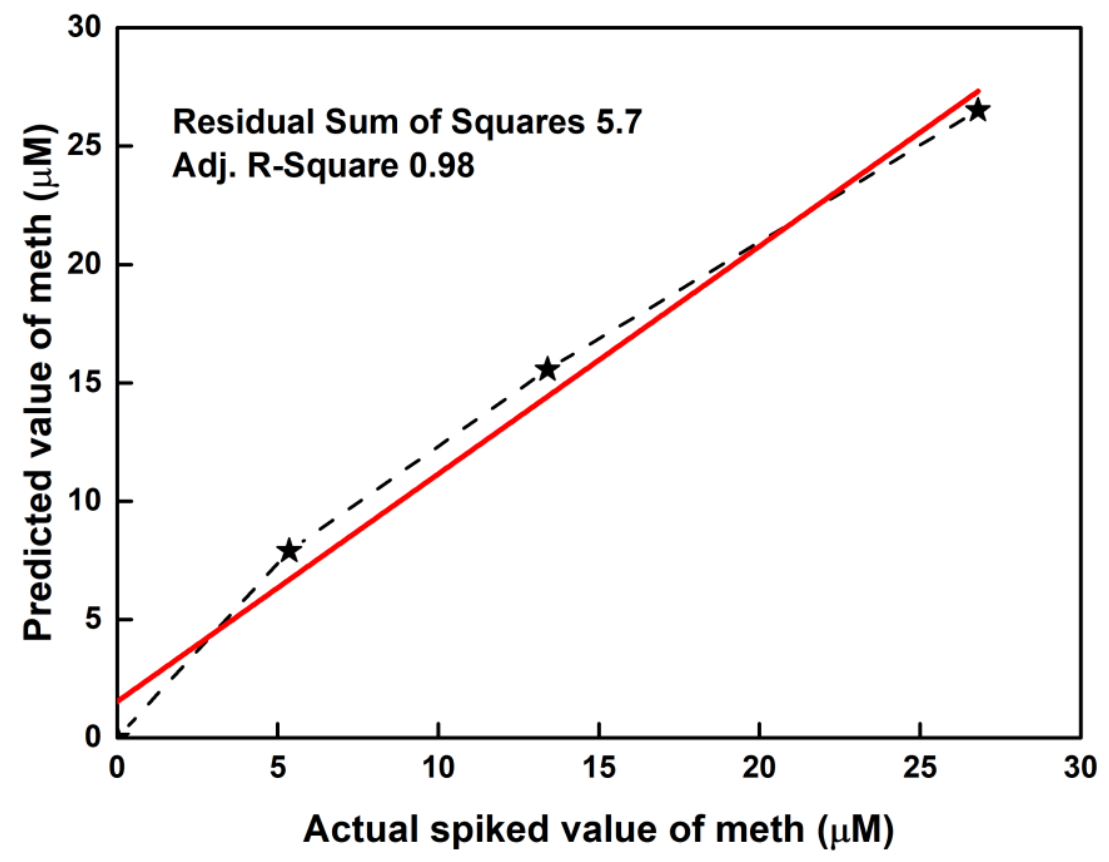

Figure 5.17 Graphical representation of spiked vs predicted values correlation in undiluted saliva. The correlation is linear and beyond this concentration range the sensor response saturates. The RSS and Rsquared value indicate that the model fit is good with immense scope for improvements.

The recovery of meth in the unknown samples were calculated after referencing the signals against those obtained in buffer to elucidate the amount of meth the sensor is able to detect in the salivary matrix and it was found that the average recovery rates $\sim 69 \%$ were higher than those obtained when the collection method was passive drooling of whole saliva directly into a tube (average recovery 49\%) suggesting that the two collection methods had a significant impact on the assay response. In other words, the signal suppression in saliva media collected via Quantisal was less $(\sim 30 \%)$ compared to those collected via passive drooling $(\sim 50 \%)$ which resulted in more signal suppression due to reasons explained previously. Figure 5.18 illustrates the relationship between the two different saliva collection methods and their suppressed sensitivities in comparison to buffer. 


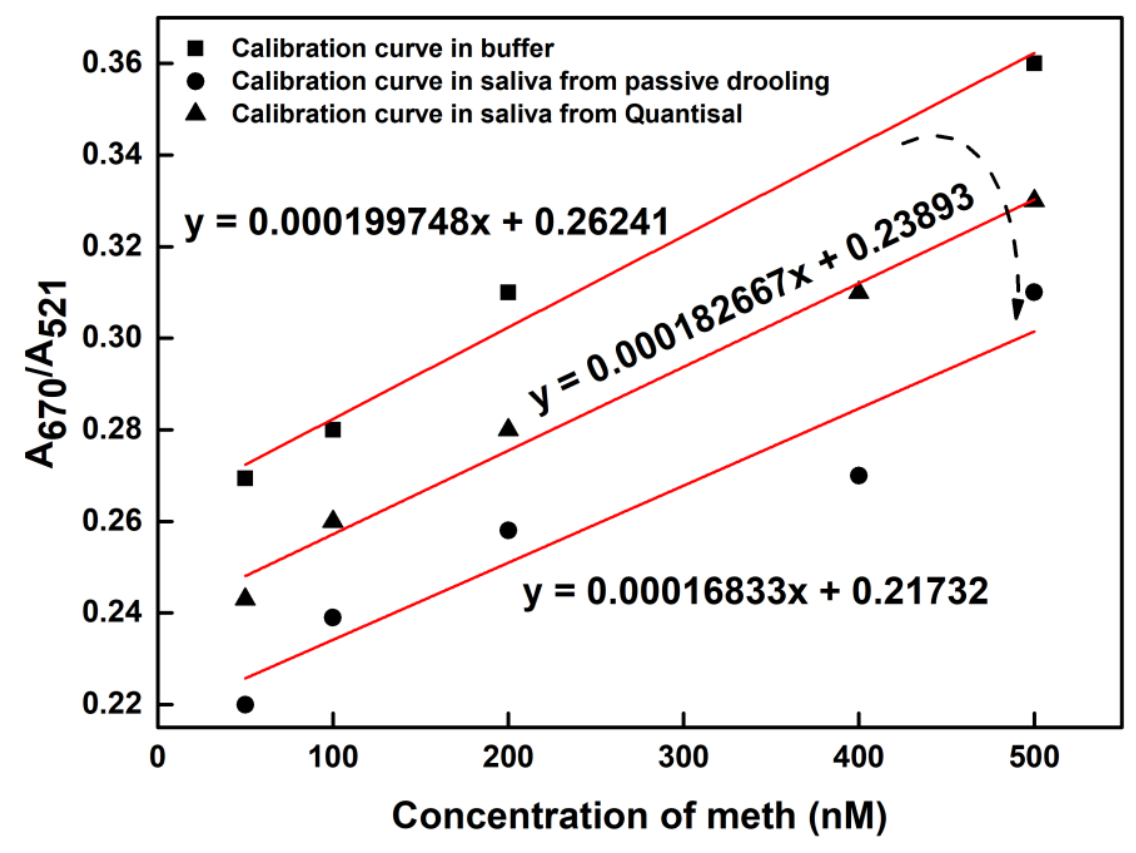

Figure 5.18 Illustration of the signal suppression in saliva medium collected via two different methods, Passive drooling and Quantisal methods. Sensitivity of response obtained with both methods were compared against that in buffer and as can be seen, the passive drooling method results in greater signal suppression compared to Quantisal collected saliva.

\section{Interference studies in oral fluid donated by healthy volunteers}

Interference studies with different compounds showed that the meth aptamer responded strongly to meth but also significantly to structurally very similar compounds such as MDMA and very slightly with MDA (could be due to the concentration in the samples and due to MDA itself because it is a primary amine like amphetamine). Interference signals from amphetamine, ephedrine, pseudoephedrine was relatively low compared to meth, MDMA and MDA.

Trials with ESR involving different interfering compounds in saliva showed no interference for compounds such as caffeine, ibuprofen, colchicine, clobazam etc at biologically relevant concentrations.

Samples containing mixture of interferants with and without meth were also tested to mimic the real-world scenario. Initial trials with high concentrations of 8-10 interferants proved unsuitable 
and led to sensor failure due to an overall effect of the ionic strength of all interferants which led to charge screening of the colloid. However subsequent trials where low concentrations were used proved difficult to obtain a response owing to the intrinsic limitation of our sensor in detecting low nanomolar levels of target. Therefore, groups of samples containing not more than 3 different mixtures of interferants were used with concentrations in the mid nanomolar range (100 ng/mL) which was suitable for obtaining a response for the presence of analyte while did not disrupt the colloidal stability. ESR provided with the samples containing a mixture of different compounds which were unknown before testing. The response obtained with the unknown samples $\mathrm{A}-\mathrm{F}$ can be found in Figure 5.19 and the details of the unknown samples can be found in Table 5.5.

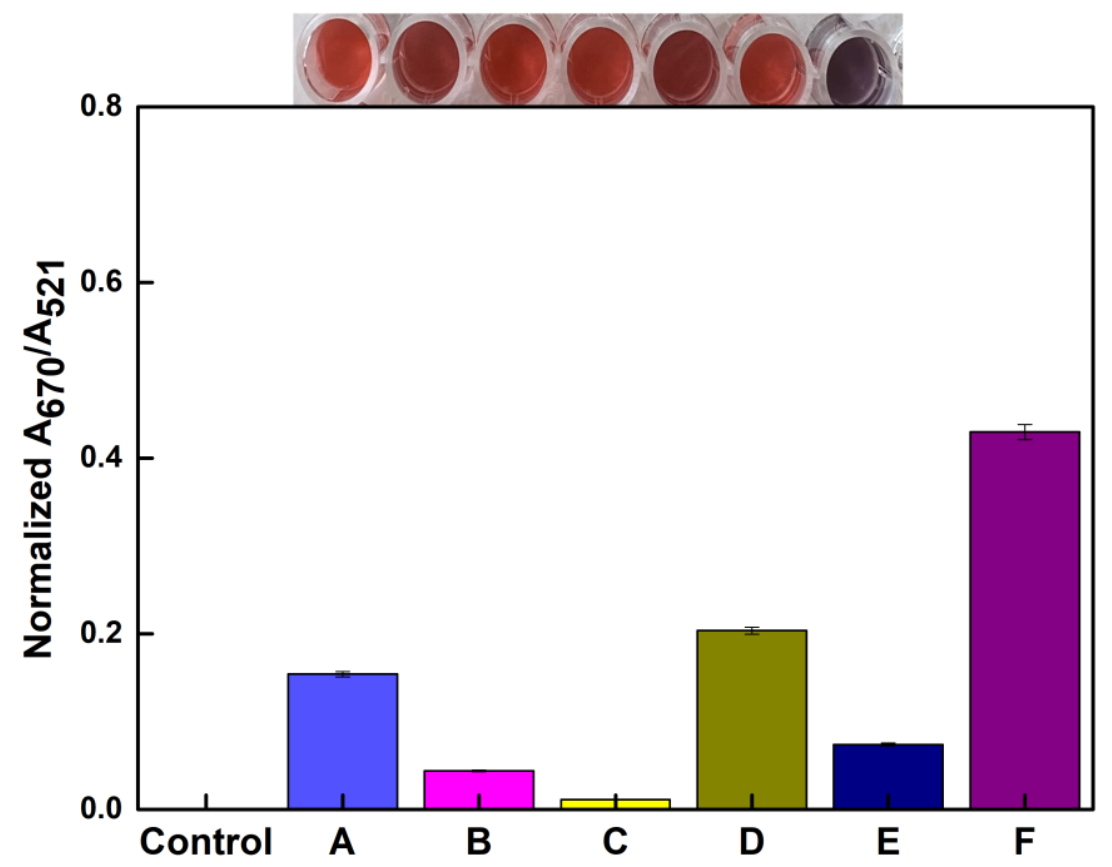

Figure 5.19 Specificity examinations of unknown mixtures of interfering molecules in saliva with the 34mer dissociation AuNPs assay 
Table 5.5 Details of the sample mixtures containing a mixture of 3 drugs (each at $100 \mathrm{ng} / \mathrm{mL}$ )

\begin{tabular}{|l|l|}
\hline Sample ID & Constituents (each at $100 \mathrm{ng} / \mathrm{mL}$ ) \\
\hline A & MDA, ibuprofen, caffeine \\
\hline B & Ibuprofen, amphetamine, caffeine \\
\hline C & Clobazam, colchicine, \\
\hline D & Meth, ibuprofen, amphetamine \\
\hline E & Colchicine, pseudoephedrine, ephedrine \\
\hline F & MDMA, meth, clobazam \\
\hline
\end{tabular}

In order to resolve and identify which compounds caused the interference, samples containing single suspected compounds were also tested as shown in Figure 5.20. As found previously in buffer, the 34-mer aptamer responded to meth and MDMA significantly. However, no interference signal was obtained with other potential and commonly found compounds such as amphetamine, precursor pseudoephedrine, commonly occurring compounds which may be found in saliva samples such as caffeine, ibuprofen etc. 


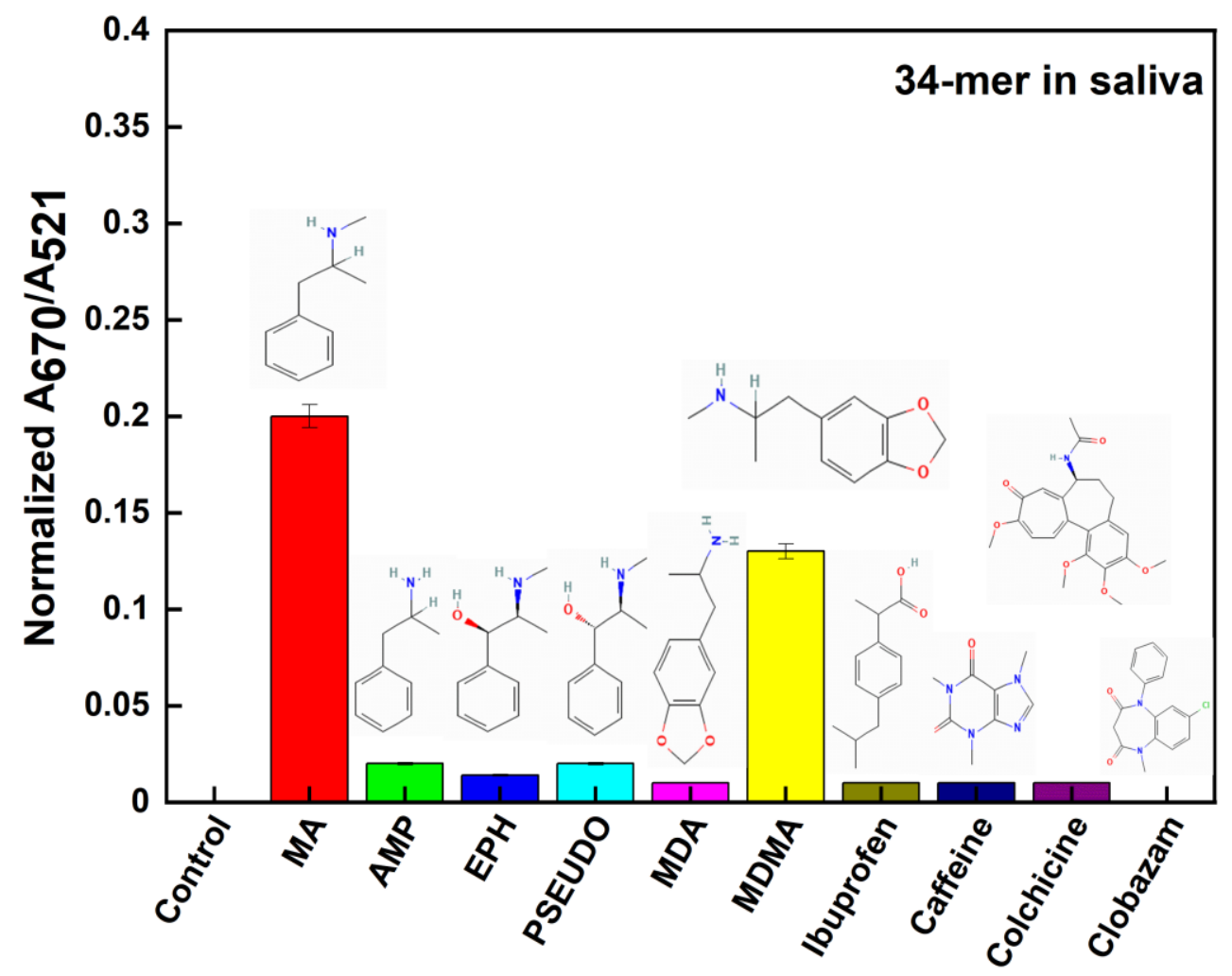

Figure 5.20 Specificity examinations in saliva samples containing single interfering compound.

In the next section, sensing responses of AuNPs based SELEX selected aptamers in saliva has been discussed. Error bars represent standard deviation of the mean of three measurements.

\section{Colorimetric sensing of meth in saliva with aptamers selected via AuNP SELEX}

The response of the new generation SELEX selected sequence, the 81-mer and it's truncated version, the 42-mer was recorded in saliva. As reported previously, the sensing responses were recorded to detect meth spiked both after and before dilution to evaluate the matrix effect and to assess the actual response closest to mimicking the real-world scenario respectively. Salt optimization studies of the 81-mer and truncated 42-mer aptamers can be found in Figure 5.21 and 5.22 respectively. The optimal salt concentration was found to be $48 \mathrm{mM}$ and $42 \mathrm{mM}$ respectively, consistent with the idea that longer aptamers once adsorbed impart a higher protection against salt induced aggrgetaion. ${ }^{206}$ 


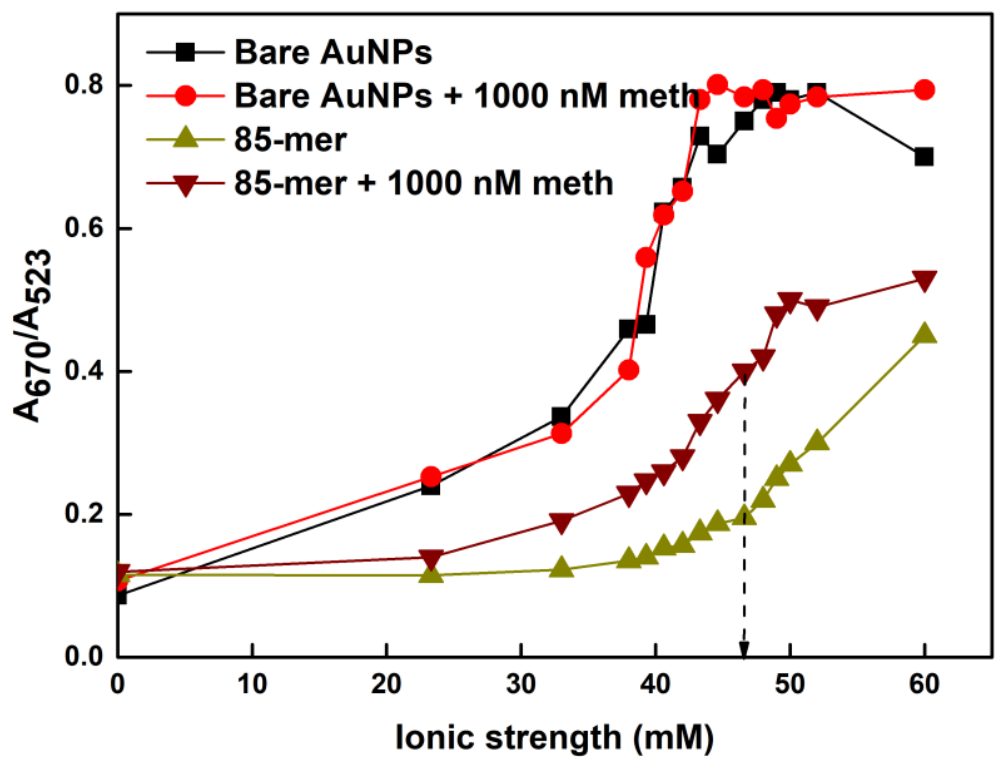

Figure 5.21 Optimum salt concertation/ionic strength needed for the optimum response of the colorimetric sensor in saliva was found to be $48 \mathrm{mM}$, depicted by the black arrow. 


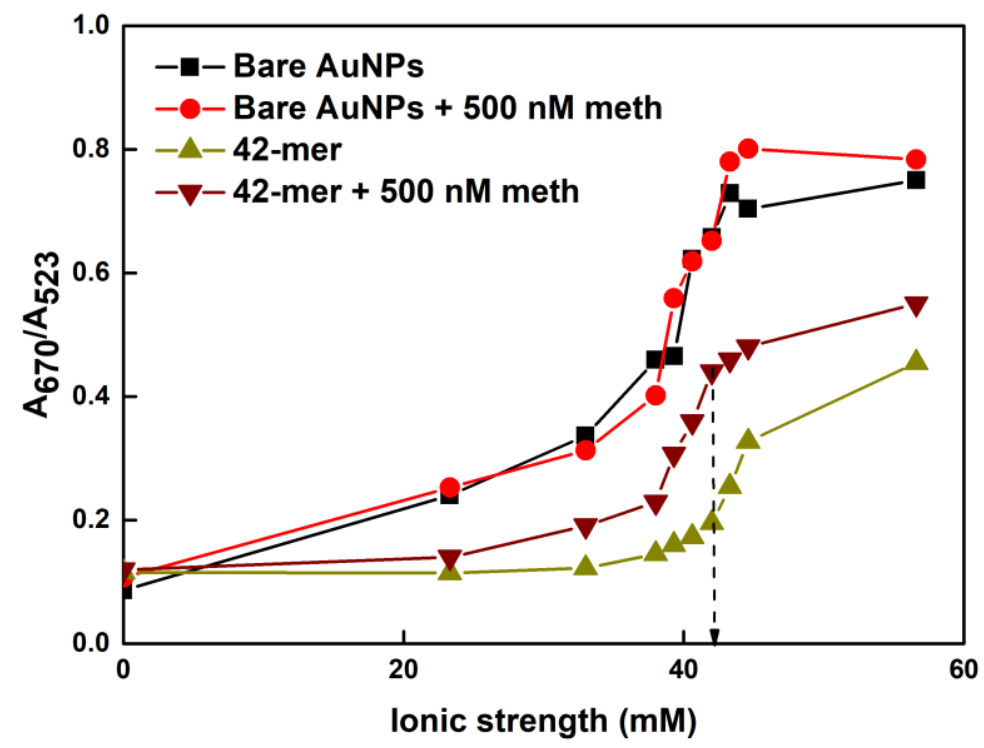

Figure 5.22 Optimum salt concertation/ionic strength needed for the optimum response of the colorimetric sensor in saliva was found to be $42 \mathrm{mM}$, depicted by the black arrow.

Figure 5.23 shows the colorimetric aptasensors response with the 42-mer in meth spiked saliva (after dilution and pre-treatment) in the concentration range 0-1000 $\mathrm{nM}$. The linear range was found to be between 20 - $400 \mathrm{nM}$ of meth with an R squared value 0.95. The calculated LOD was found to be $60 \mathrm{nM}$ ( $240 \mathrm{nM}$ in undiluted saliva) which is much lower than the LOD $161 \mathrm{nM}(\sim$ $644 \mathrm{nM}$ in undiluted saliva) obtained with the 34-mer selected via traditional bead immobilized affinity-based SELEX. 


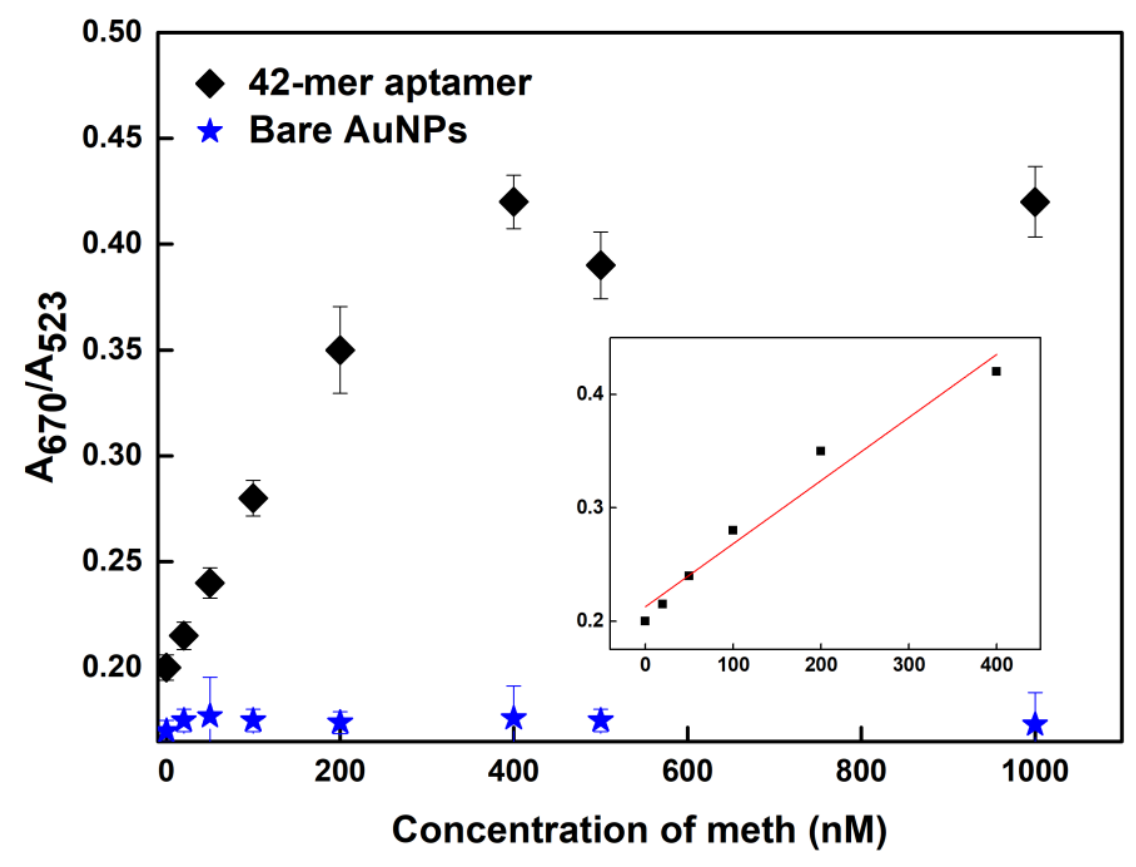

Figure 5.23 Colorimetric response of 42-mer aptamer in saliva that was spiked with meth after dilution and pre-treatment. The response is compared with bare AuNPs control to rule out non-specific adsorption of meth. Inset showing the linear range. Error bars represent standard deviation of the mean of three measurements.

In order to evaluate the signal suppression and the matrix effect of diluted saliva, the signals obtained at spiked meth concentrations of 100, 300 and $500 \mathrm{nM}$ of meth with the 42-mer in saliva were referenced against that obtained in buffer using the calibration equation $y=7.4 \times 10^{-4} x+$ 0.223 (Table 5.6). It was found that the recovery rates were consistent with those found previously with the 34-mer and on an average there was $50 \%$ signal suppression in saliva. 
Table 5.6 Calibration of the obtained signal from the 42-mer aptamer in saliva samples that were spiked with meth after dilution/pe-treatment

\begin{tabular}{|l|l|l|l|}
\hline $\mathrm{A}_{670} / \mathrm{A}_{523}$ ratio & Spiked Concentration $(\mathrm{nM})$ & Found meth $(\mathrm{nM})$ & $\%$ recovery \\
\hline 0.26 & 100 & 50 & 50 \\
\hline 0.36 & 300 & 185 & 62 \\
\hline 0.37 & 500 & 199 & 40 \\
\hline
\end{tabular}

Figure 5.24 shows the summary of colorimetric response of parent aptamers 81-mer and 75-mer selected via AuNPs aggregation-based SELEX and traditional bead immobilized affinity-based SELEX respectively, and their truncated versions 42-mer and 34-mer respectively against meth concentrations ranging from $0-2 \mu \mathrm{M}$ spiked in saliva after treatment. This concentration range was selected to accommodate for the response of the long aptamers which were unable to respond to low nanomolar concentrations of meth. 


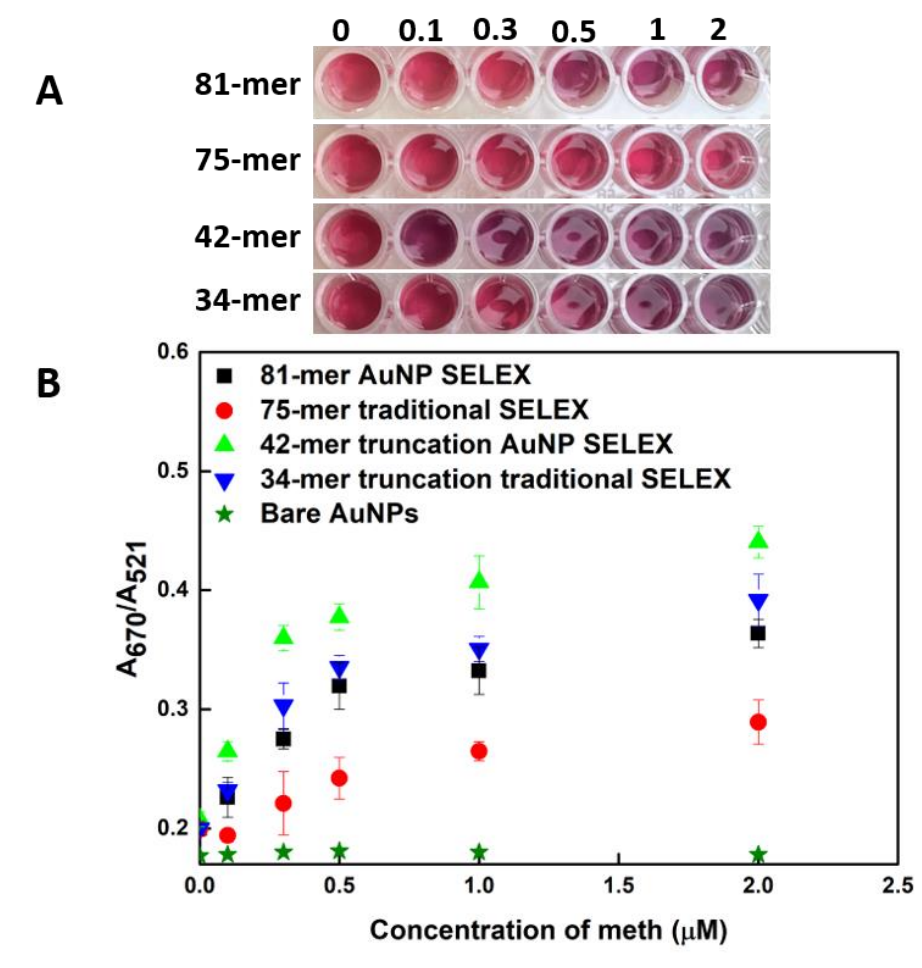

Figure 5.24 (A) Visual (B) Graphical comparison of sensing responses of traditional vs AuNP SELEX selected aptamers in saliva that was spiked with meth after dilution and pre-treatment. Error bars represent standard deviation of the mean of three measurements.

Alternatively, collected saliva samples were spiked with 1, 3, 5, 10 and $20 \mu \mathrm{M}$ of meth prior to dilution and pre-treatment. It was found that 42-mer had the most promising response among the other sequences followed by 34-mer and 81-mer which have almost comparable response and the 75-mer which is least responsive to meth in saliva which yet again highlights the effect of selection strategy on the performance of the aptamer (Figure 5.25). The obtained signals of the 42-mer in saliva were referenced against that obtained in buffer using the equation $y=7.4 \times 10^{-4} x+0.223$. It was found that the recovery rates were very low $<1 \%$ (Table 5.7) when compared to the amount of meth spiked in the saliva initially (before dilution and pre-treatment). This was expected because a lot of meth (4 times) is lost due to dilution. Therefore, for a successful sensing response the meth present in saliva needs to be at a high concentration apparently above the cut-off that is required to account for the dilution. But high concentrations of meth in saliva is not biologically expected always. This of course is challenging and may be regarded as a limitation of the present work because dilution is necessary for application in AuNP assays. 


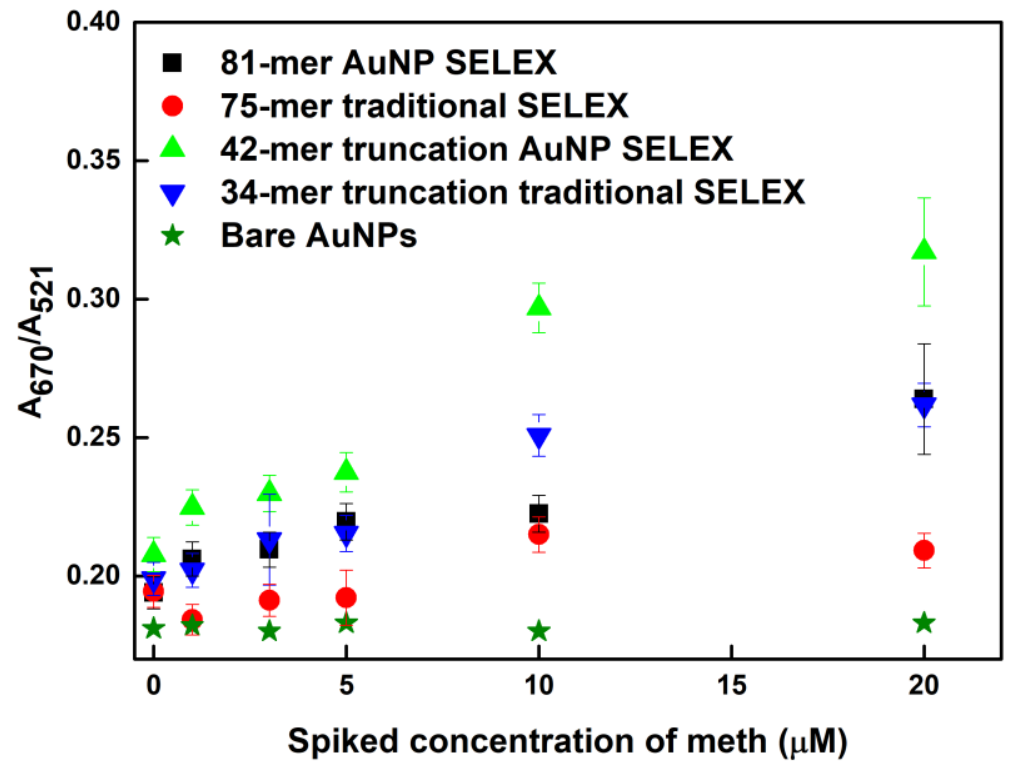

Figure 5.25 Colorimetric response obtained from old vs new SELEX selected aptamers at meth concentrations spiked prior to pre-treatment of saliva samples. Error bars represent standard deviation of the mean of three measurements.

Table 5.7 Calibration of the obtained signal from 42-mer aptamer after spiking meth prior to pre-treatment in saliva

\begin{tabular}{|l|l|l|l|}
\hline A670/A523 ratio & Spiked Concentration $(\mu \mathrm{M})$ & Found meth $(\mathrm{nM})$ & $\%$ recovery \\
\hline 0.237 & 5 & 18.93 & 0.37 \\
\hline 0.297 & 10 & 100 & 1 \\
\hline 0.317 & 20 & 128 & 0.64 \\
\hline
\end{tabular}




\subsection{Chapter conclusion}

The challenge of using oral fluid as a diagnostic fluid in AuNPs based aggregation assay has been demonstrated in this chapter. A detailed study of interference of biomolecules and electrolytes present in saliva was demonstrated and then successfully mitigated for the purpose of detecting meth at biologically relevant concentrations.

Both chemical and physical methods to extract or remove proteins were investigated to determine the most suitable protein removal technique compatible with the assay as well as keeping in mind the POC application requirements. It was found that a sequential syringe filtration using a GD/X PES filter membrane and positively charged Nylon membrane was most effective in reducing levels of overall protein in saliva and this was confirmed by both DLS and UV-Vis studies. Sensing studies in saliva were successfully conducted with some extent of interference from salivary matrix which was evident from the average signal suppression of about 40-50\% observed when referenced against the signals obtained in buffer. The detection was always suppressed in saliva.

Validation trials with samples obtained from collaborators was done extensively and most trials were successful in getting successful signals in saliva spectroscopically when the concentrations were above $800 \mathrm{ng} / \mathrm{mL}$ and even visually at higher concentrations. These results show immense promise in the application of saliva for the detection of drugs of abuse. The level of detection obtained with the 34-mer aptamer spectroscopically was $96 \mathrm{ng} / \mathrm{mL}(644 \pm 3 \mathrm{nM})$ in undiluted saliva which is above the cut-off level of $25 \mathrm{ng} / \mathrm{mL}$ for meth in oral fluid required for the initial screening tests at workplaces and roadside. Due to the necessity of dilution in the present work, it was challenging to obtain the required detection level below the cut-off value.

Interference studies showed that the 34-mer meth aptamer was responsive to meth as well as MDMA and MDA. Although the response was not as strong as in the presence of meth, it was difficult to ascertain the absolute specificity solely towards meth. More importantly, the interference from these interferants were not very surprising because of the striking structural similarity of these closely related compounds. The 34-mer aptamer gave no interference response towards the presence of other non-related compounds such as caffeine, ibuprofen, paracetamol etc. which are also some of the commonly found compounds in oral fluid. 
Lastly, the response of a new generation SELEX, AuNP SELEX selected aptamer for meth was compared with the 34-mer aptamer which had been selected via the traditional affinity-based SELEX involving bead immobilized target. As discussed in Chapter 4, the sequence gave promising results when compared to the 34-mer in saliva. The LOD achieved with the 42-mer aptamer was $240 \pm 3 \mathrm{nM}(\sim 35.8 \mathrm{ng} / \mathrm{mL})$ which is also above the cut-off value of meth required to be detected in saliva but there has been a $\sim 3$ fold improvement of in the detection level with the AuNP SELEX strategy. This finding was extremely promising and highlights the possibility of application of the current assay in real saliva with biologically relevant detection levels of meth in the future. Overall, it can be concluded that there is scope for further improvements around aptamer developement as well as saliva processing techniques to include electrolyte removal steps as well as more advanced protein removal techniques so that dilution can be minimized. Despite the challenges and limitations, demonstration of a successful colorimetric sensor in saliva was achieved. 


\section{CHAPTER 6}

\section{Fabrication and validation of a cost-}

effective and sensitive handheld device for methamphetamine detection 


\subsection{Abstract}

There is a widespread need for reliable and accurate quantification of colorimetric signals on-site. This has motivated the development of a handheld portable light meter device that can reliably transduce the aptasensors response into absorbance, facilitating the determination of analyte concentrations on-site. This is crucial due to the prevalent unreliability and sensitivity problems of the conventional drug test kits as mentioned in Chapter 1. Therefore, a portable detector system for meth has been developed to in this chapter to quantify the visual colorimetric response obtained from the meth aptasensors which may be used for on-site application purposes. The fabricated light meter device response was validated against a standard UV-Vis spectrometer to confirm reliability. A 42-mer meth aptamer selected via METH SELEX, described in Chapter 4 was used for aptasensing purposes. Finally, a portable and cost-effective handheld detector device whose sensitivity was found to be comparable to the well-established UV-Vis spectrophotometry-based instrument. The easy to use device could potentially serve as a prototype for a commercial device. 


\subsection{Introduction}

While meth abuse continues to be a concerning situation in NZ as stated in the previous chapters, there is an urgent need for roadside and workplace drug evaluation devices that are highly accurate, sensitive, and reliable. Such point-of-care devices would not only facilitate widespread testing but also provide cost-effective diagnosis and resource utilization. As demonstrated in previous chapters the promising performance of colorimetric aptasensors in detecting meth in oral fluid led to the idea of addressing these demands by quantifying colorimetric responses. Quantification is preferred for accuracy and reliability.

Recent news articles suggest that in 2019, meth drugged driving has resulted in more deaths in 2019 compared to alcohol in New Zealand. ${ }^{207}$ Of the limited reports in the literature regarding the specific effect of meth impairment on driving, a study by Silber et al. ${ }^{208}$ reports the effect on driving, under the influence of meth. The study was conducted using healthy volunteers, dosing them with ethically controlled quantities of meth before subjecting them to a performance testing session on a driving simulator. The reported effects of meth on simulated driving compared to the control subject involve releasing of brakes inappropriately when stopping, driving too fast for the traffic conditions and travelling slower on the freeway in an emergency. Since the levels of meth administered were relatively low compared to what might be in an actual scenario, the authors extrapolate to the effect that a higher impairment may have had. Similarly, Bosanquet ${ }^{209}$ studied and compared meth users with non-meth users again evaluating the performance in a driving simulator. The results were consistent with those found earlier by Silber et.al. and demonstrated an increased tendency to risky driving behaviour compared to controls. Studies by Stough and Reamaker et al. ${ }^{210}$ also followed a similar procedure reporting increased aggression in groups of meth users compared to control subjects. Such culpability and behavioral studies on a driving simulator provides enough evidence to delineate the risk associated with drugged driving.

Owing to the shortcomings of the traditional and existing methods for detecting meth, colorimetric aptasensors already described in Chapter 1 are particularly attractive in terms of simplicity and high sensitivity of detection. The detector there typically used for quantifying responses is the UVVis spectrophotometer. The instrument is obviously bulky and impractical for on-site applications. Therefore, it is important to look for portable hand-held detectors which can reliably detect meth at biologically relevant concentrations on site in order to quantify a visual yes/no response reliably. 
Several examples already exist in literature which have combined AuNP colorimetric biosensors with portable detection systems or smartphone camera-based image processing applications. ${ }^{125,123,211}$

In this chapter, the design and application of a prototype hand-held device integrated with a sample holder, a $520 \mathrm{~nm}$ LED and a light meter device which measures the changes in the transmittance induced by the meth in sample is discussed. The principle as described in previous chapters is based on the meth induced desorption of aptamers and the subsequent aggregation of unprotected AuNPs leading to a visual colour change, and a transmittance value that is directly proportional to the degree of aggregation.

\subsection{Results and discussion}

\section{Design and fabrication of portable light meter device}

\section{Requirements for the device}

1) Absorption spectroscopy: AuNPs exhibit unique optical properties owing to surface plasmon resonance. Since the detection is based on absorption spectroscopy with a single wavelength LED i.e. $520 \mathrm{~nm}$, this wavelength must be resonant with the frequency of oscillation of conduction electrons of AuNPs for them to absorb the wavelength and facilitate accurate determination of absorbance from the transmitted intensity.

2) Single channel detector: A single channel detector only detects photons of a particular wavelength that are transmitted through the sample. In this case, the active sample is AuNPs that absorb the incident wavelength from the $520 \mathrm{~nm}$ LED and the transmitted light intensity is then detected by the detector. A detector must be stable and provide reproducible data.

3) Sample holder: A sample holder is a necessary component that is required for holding the sample. A sample holder should be non-reactive with the sample and clean to avoid scattering.

4) Stability: Stability is a foremost requirement of any instrument which determines repeatable and reproducible results. The long-term stability of a detector is important for 
consistent high-quality measurement. We define stability as the ability of the detector to maintain the measured property at a constant value over time.

5) Cost of device: One of the characteristic attributes of a POC device is its cost-effectiveness. The total cost of the fabricated device can be divided into cost of the detector + cost of the coupled device prototype which was $60+16=76$ NZD.

\section{Design of the prototype device}

The proposed design was executed by the School of Chemical and Physical Sciences workshop. A power bank or laptop can be used to power the LED. Data was recorded in UNI-T's phone application iENV.

\section{Materials}

The LED was purchased online from RS Components (3.55 V green LED with dominant wavelength $520 \mathrm{~nm}$ ). The Light meter device was purchased online from Hvactools (Product name UNI-T UT383BT Bluetooth mini digital light meter LUX). Vials were cut out/extracted from the multi-well plates used during the sensing experiments discussed in the previous chapters.

\section{Resolving a stable baseline}

The device was made to facilitate stable data collection. Without a proper housing, it was observed that the LED would move due to inevitable handling errors, resulting in inconsistent values that did not permit reliable interpretation. It was hypothesized that a proper housing would lead to stable readings of the transmitted intensity. The designed light proof attachment concept can be seen in Figure 6.1. 


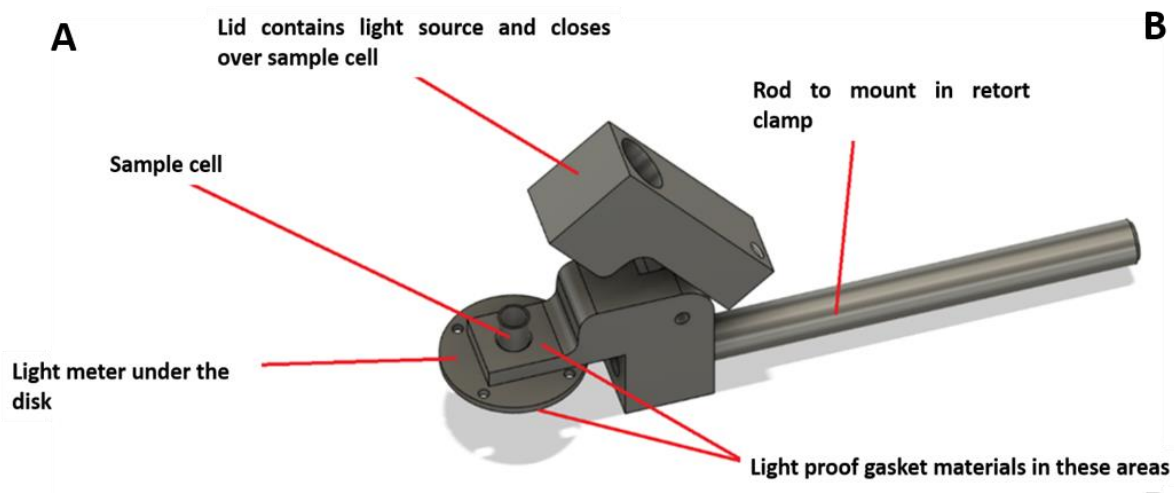

B
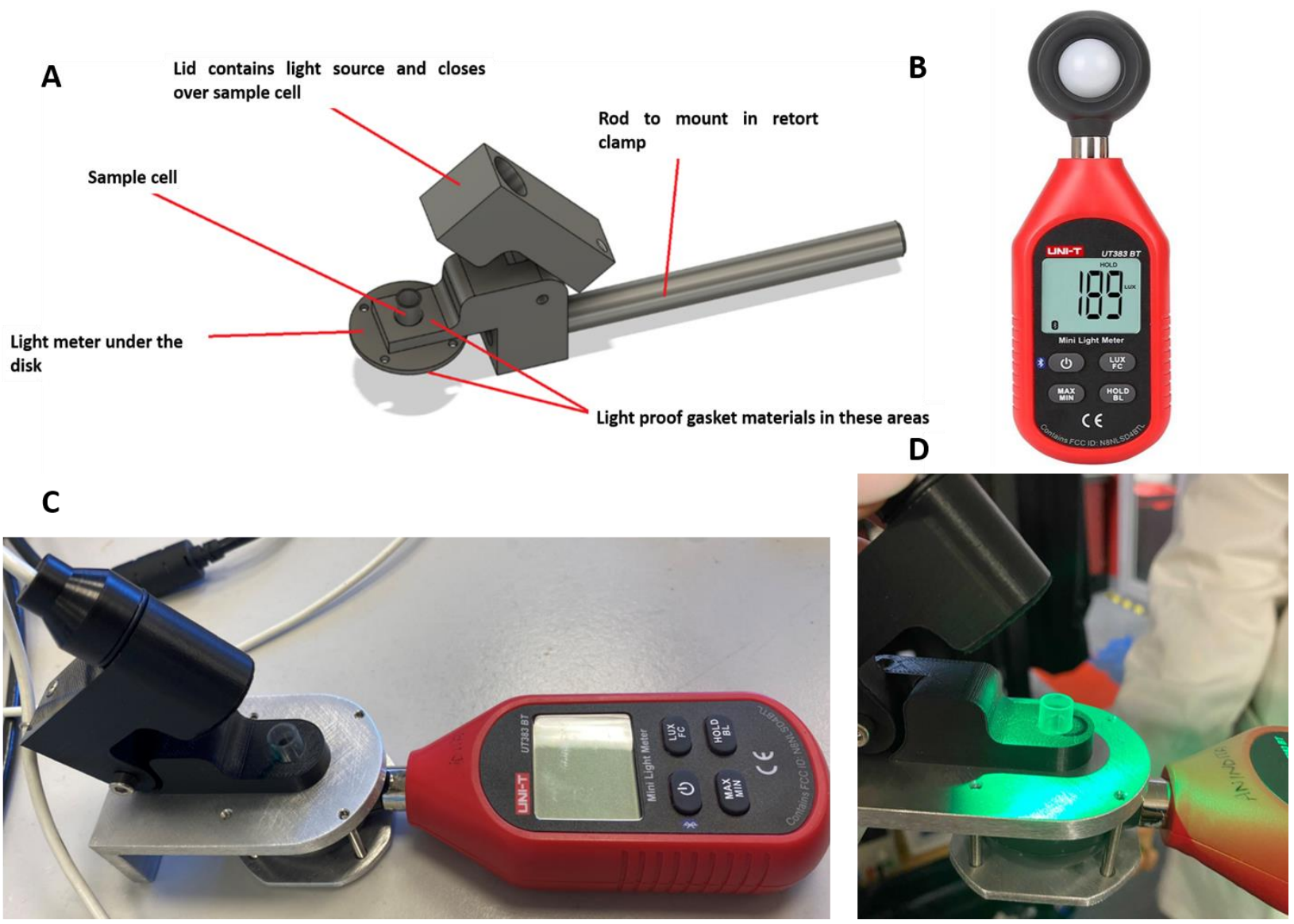

Figure 6.1 (A) Concept of the light proof attachment containing the light source and sample holder, parts labelled. (B) The mini Bluetooth digital light meter C) The final device that was made by attaching the light proof attachment with the digital light meter detector. D) Close-up image of the LED attached to the lid and closing over the sample vial.

The vials made by the workshop had a lot of minute cracks probably due to the extraction and polishing process after extraction from the plate. This led to an extremely noisy and unreliable baseline because of the vial due to unpredictable scattering of light, which lead to irreproducible readings every time the vial was removed and placed back into the device. Removing the vial was necessary because there were different sample concentrations in different vials. Moreover, the nature of the assay which has multiple incubation steps necessitates removal of vials in order to make the process time efficient. However, this was proving difficult due to the scattering problem arising from the vials such that each vial had their own unique defects and the problem was even worse because even in a single vial, there were positional defects. It was hypothesized that the 
problem arising out of errors from changing orientation of the vial could be resolved if the vial be placed at the exact same position every time it is removed and placed back.

A stable baseline was therefore achieved by marking the vial when placed at a certain position to align with a marking on the device such that it was possible to place the vial in the exact same orientation and position. This resolved the problem of unstable baseline. Therefore, each vial was marked in the same way to ensure that once a vial was removed it could be put back into the exact same position and same stable baseline to start from.

\section{Stability studies of light meter device}

In order to investigate the stability of the light source and the detector, a sample of AuNPs in the sample vial was placed in the sample holder. A reading of transmitted intensity was noted at time $\mathrm{t}=0$ and left for 30 minutes with intensity readings noted every 5 minutes. As shown in Figure 6.2, the readings were stable over a span of 30 minutes. The sample vial was also taken off and put back in to confirm reliability of the method discussed in the previous section to eliminate scattering induced instability. 


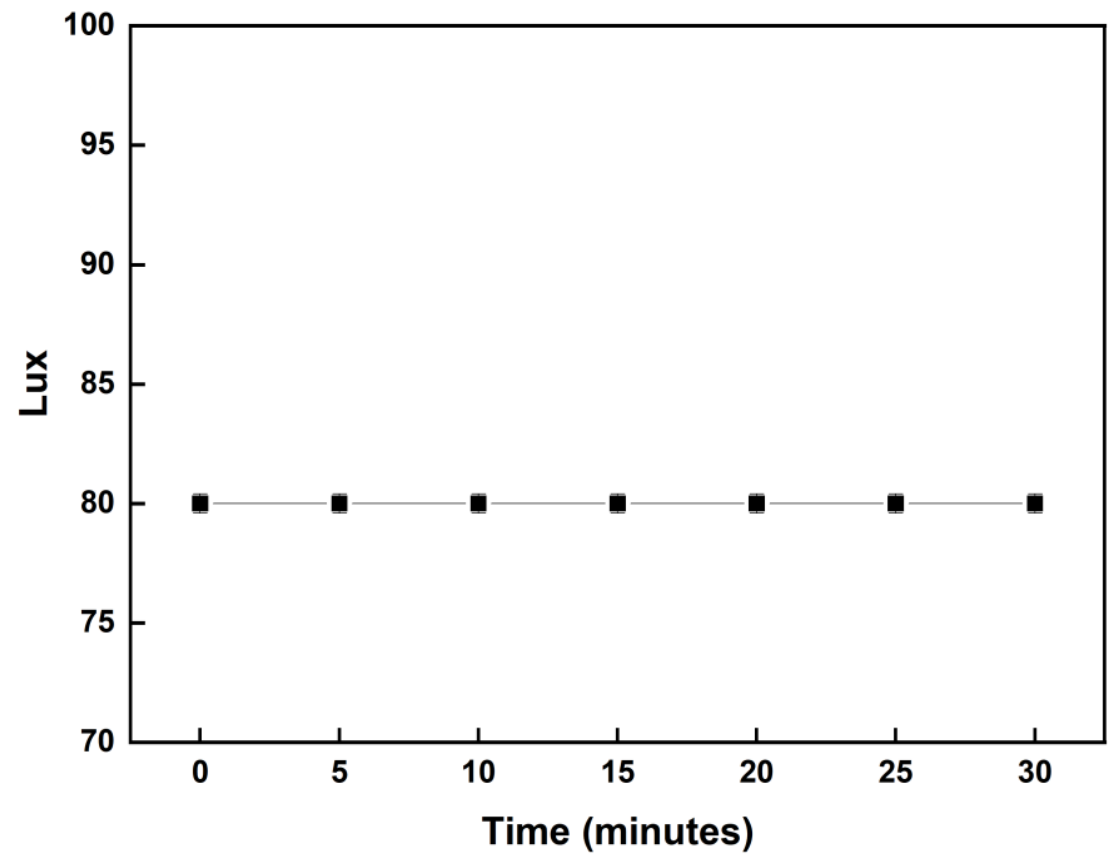

Figure 6.2 Graphical illustration of stability study of the light source and detector over time to confirm the stability of the set up. Error bars represent the standard deviation of mean of three measurements.

Once, a stable working system was achieved, colorimetric aptasensing experiments were conducted. The 42-mer aptamer was selected as the probe for performing the biosensing experiments, because, as described in Chapter 4, this was the most promising in efficient detection of meth with an LOD of $10 \mathrm{nM}$ in aqueous solution. The 42-mer could be used to adsorb to AuNPs following the dissociation assay as it was selected using the modified AuNP SELEX which accounted for the association/adsorption and dissociation/desorption interactions as mentioned in Chapter 4 . The meth concentrations used were $0,0.1,0.3,1$ and $2 \mu \mathrm{M}$. Following the procedure described in Chapter 3, the aptasensing experiment response was recorded first by the light meter device and then by the plate reader as shown in Figure 6.3.

\section{Calculation of the absorbance}

The reading of each sample vial without the sample gave us the light intensity $\mathrm{I}_{0}$ in terms of lux which measures light intensity/luminous flux per unit area $\left(\mathrm{Lux}=1 \mathrm{lumen} / \mathrm{m}^{2}\right)$. These values were recorded. 
After addition of $100 \mu \mathrm{L}$ of AuNPs + aptamer followed by $12 \mu \mathrm{L}$ of buffer/meth, the sample vial reading was recorded providing the baseline reading at $0 \mathrm{NaCl}$. This was necessary to get the actual reading of the intensity arising after addition of $\mathrm{NaCl}$. Since each vial was different, it was necessary to consider the baseline of each vial separately so that the actual intensity could be obtained by subtracting the baseline signal from the final intensity reading.

The intensity of the samples was recorded after addition of $8 \mu \mathrm{L}$ of $\mathrm{NaCl}$ which resulted in a colour change and an increase in the transmitted light intensity, signifying aggregation due to presence of meth in samples other than the control.

The signal obtained by subtracting the respective baseline of each vial was used to compare the response among the samples. It was seen that the transmitted light intensity increased with concentration.

Next, the values were converted into absorbance by using the formula

$$
A=-\log T, \text { where } T=I / I_{0}
$$

The absorbance was inversely proportional to the concentration, consistent with increasing aggregation caused by increasing desorption of aptamers with increasing concentration of meth was indicated by a decrease in the absorbance at $520 \mathrm{~nm}$. 


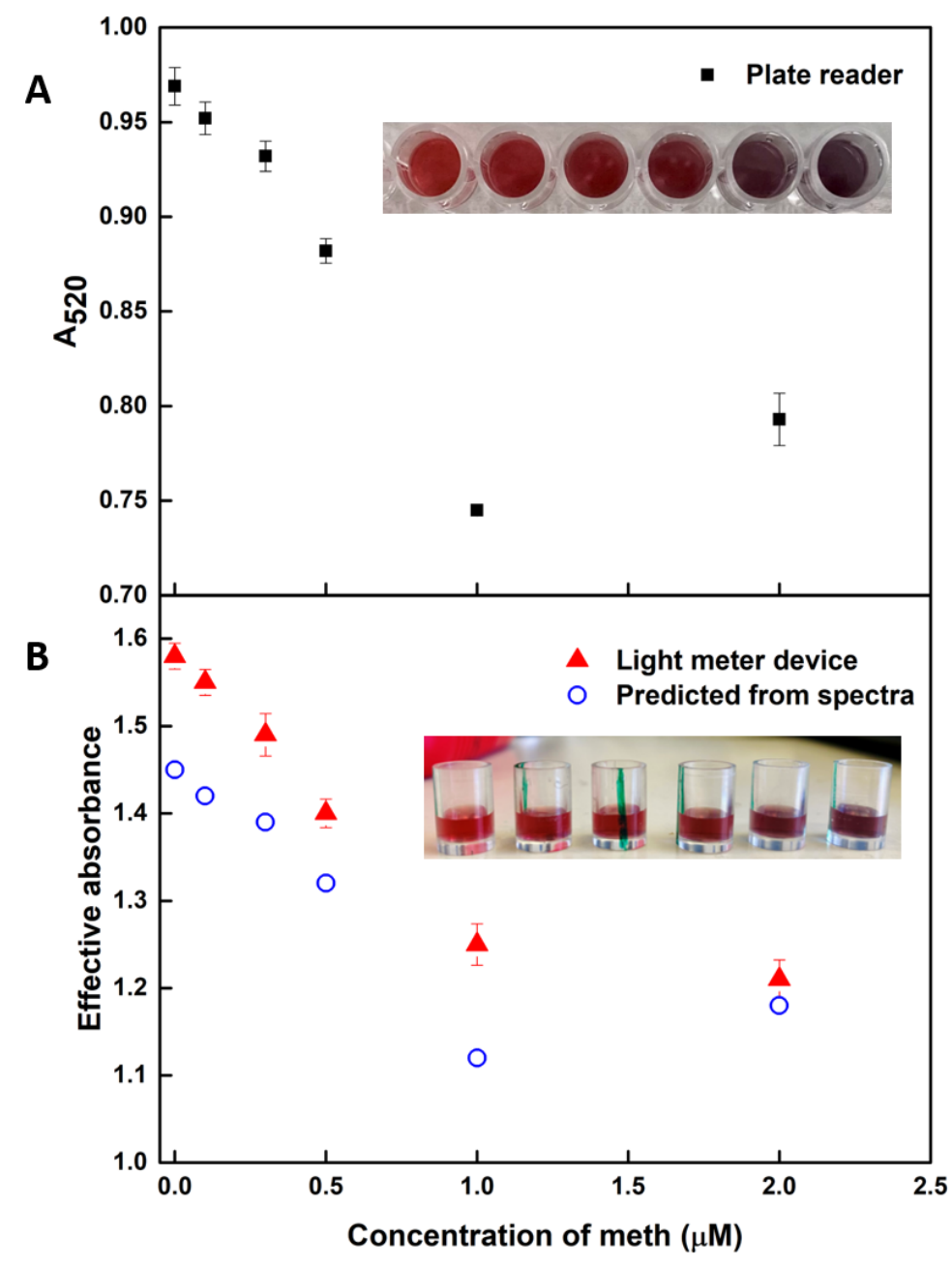

Figure 6.3 (A) Colorimetric response of the 42-mer meth aptasensor in terms of absorbance at $520 \mathrm{~nm}$ obtained from the standard plate reader instrument. Inset shows the visual colour response over the concentration range 0-2 $\mu \mathrm{M}$. (B) Colorimetric response obtained from the light. Error bars represent standard deviation of the mean of three measurements.

Theoretically, the absorbance of the same sample measured by different devices (spectrometers/colorimeters) should return the same values, however, in practice this might not be the case due to the differences in the light source, detectors and optics.

The difference in absorbance value levels seen in Figure 6.3 for the same sample when measured with the plate reader and light meter device can be accounted for by considering the overlap of the absorption spectrum of a sample from a spectrophotometer with that of the LED emission spectrum, and calculating the effective absorbance. The reason the absorbance values from the 
light meter device do not match the plate reader UV-Vis is that the former values are not exactly the absorbance at $520 \mathrm{~nm}$, but rather an effective absorbance over a wider range. It is important to account for this difference to prove/validate that the values displayed by the device accurately reflect the absorbance of the sample.

\section{Calculation of the effective absorbance from overlapping integral analysis}

The following procedure was used to determine the effective absorbance:

From the UV-Vis absorption spectrum, the ratio of transmitted light intensities to the input intensity can be obtained i.e. $\frac{I}{I_{0}}(\lambda)=10^{-\mathrm{O} . \mathrm{D}}$, dependent on wavelength $(\lambda)$.

The LED light emission spectrum from the specification sheet shows the relative $\mathrm{I}_{0}$ dependant on wavelength.

If $\mathrm{I}_{0}=\mathrm{n}(\lambda)$ is the emission spectrum of the LED spectra, the transmitted intensity $\mathrm{I}(\lambda)$ after passing through the sample can be found by

$\frac{I}{I_{0}}(\lambda) \times n(\lambda)=\mathrm{I}(\lambda)$

By summing over/integrating over the different wavelengths, it is possible to find the total intensity of transmitted photons i.e. $\sum \frac{I}{I_{0}}(\lambda) \times n(\lambda)=\sum \mathrm{I}(\lambda)=\mathrm{I}$

To get the effective absorbance, the number of transmitted photons I can be divided by the true number of incident photons or $\mathrm{I}_{0}$ and $\mathrm{I}_{0}$ can be represented in terms of $\mathrm{f}(\lambda) \times \mathrm{N}$ where $\mathrm{f}$ is relative number of photons and is obtained from the lamp spectra dependent on wavelength and $\mathrm{N}$ is the normalization factor.

The formula used to obtain the effective O.D therefore becomes $\mathrm{I} / \mathrm{I}_{0}=\sum_{\lambda}\left(\mathrm{I} / \mathrm{I}_{0}\right)_{\lambda} \times \mathrm{n}(\lambda) / \sum_{\lambda} \mathrm{n}(\lambda)$, where $\mathrm{n}=\mathrm{fx} \mathrm{N}$ therefore $\sum\left(\mathrm{I} / \mathrm{I}_{0}\right)_{\lambda} / \sum \mathrm{f}(\lambda)$ which gives $\mathrm{I} / \mathrm{I}_{0}=-\log$ O.D.

After calculations, the effective $\mathrm{I}_{\mathrm{I}}$ obtained from this analysis was found to be 0.03 , corresponding to an effective absorbance of 1.5. This effective absorbance can be used to predict the absorbance values that should be given by the LED and can be seen in Figure 6.3 (B). It was seen that the predicted effective values were slightly different but close to those observed by the 
light meter device. This could be due to a variety of factors: such as the path length may be varied due to the incident light from the light source in the device not following a straight path but bouncing around the sample and reaching the detector without passing through the sample. Also, the detector may be saturated due to which the values are different from the predicted ones. Given that the device gives a value higher than those predicted it should be emphasized that the light which is not a collimated beam can undergo internal reflection and travel longer paths through the sample, rather than the shortest path i.e. it has a longer effective pathlength. If stray light was not going through the sample before reaching the detector, then the effective absorbance would have been reduced (which is not observed).

By calculating the effective absorbance from the overlap integral analysis, it was possible to prove that the numbers measured with the light meter device were the absorbance of the sample. Now, it can be ascertained that the light meter device can accurately correlate with the aptasensors response and serve as an on-site device that can be used to quantify the colorimetric response for meth as well as other small molecules and analytes.

\section{Comparison of the LOD}

Figure 6.4 shows the calibration curve obtained from the two devices. The linear range was between 0.1-1 uM (100 - $1000 \mathrm{nM})$. The calculated LOD was $63 \pm 2 \mathrm{nM}$ for the light meter device and $159 \pm 3 \mathrm{nM}$ obtained from the standard plate reader. Both values were promising as they were well below the cut-off $167 \mathrm{nM}$ required for the conventional drug testing standards.

Interestingly, the LOD obtained from the UV-Vis plate-reader was higher that than obtained from the light meter device which suggested the comparable and superior sensitivity offered by the portable light meter device which facilitated an improvement in the LOD. This achievement can be owed to the stability of the fabricated device which enabled reliable and sensitive detection of the colorimetric aptasensors response. 


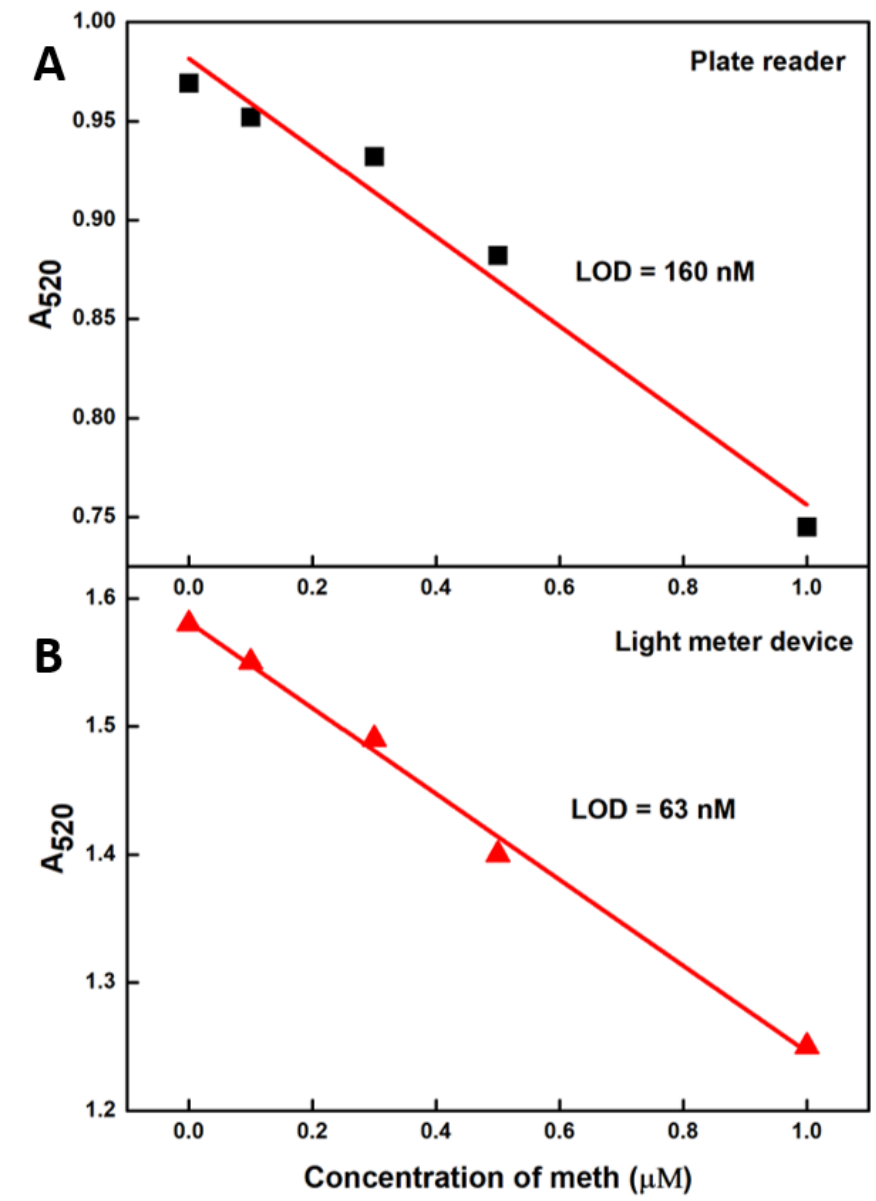

Figure 6.4 Calibration curve of the 42-mer aptasensor obtained with (A) the standard plate reader absorbance values (B) the light meter device. 


\subsection{Chapter conclusion}

In this chapter the design and development of a handheld POC friendly detector device integrated with a sample holder, LED light source and detector has been demonstrated. Validation of the signals obtained from the light meter device was achieved by analysing the LED emission spectrum and the sample absorbance spectrum from a UV-Vis spectrophotometer. It was found that the LED light meter device gave more sensitive levels of detection. A cost-effective $(<\$ 100)$ and sensitive POC friendly device was developed capable of accurately detecting meth in solution at levels below the cut-off levels of meth in biological matrices. In short, this chapter demonstrates the development of a suitable prototype with immense scope for customization and improvement. 


\section{CHAPTER 7}

\section{Conclusion and future perspectives}




\section{Conclusion}

The aim of this thesis was to optimize and develop a colorimetric aptasensor system for the detection of methamphetamine in oral fluid at legally relevant detection levels of $25 \mathrm{ng} / \mathrm{mL}$.

In Chapter 3, the optimization and development of a sensor using a methamphetamine aptamer selected by the traditional SELEX method was discussed. It was found that the competitive association and dissociation reactions between the aptamer and AuNPs in the presence of target, which form the basis of these AuNP aggregation assays, were not in a state of equilibrium. Consequently, the aptamers were found to interact with AuNPs in a time dependent manner, which hindered their target binding capabilities. This equilibrium problem was illustrated via time dependent salt titration and zeta potential studies. Moreover, it was shown that by reversing the sequence of addition of AuNPs in the assay so as to reduce the chances of aptamers getting trapped on AuNPs before binding to target, the response of the assay improved significantly. The new so called 'association assay' facilitated the detection of meth with an 8-fold improvement in the detection sensitivity. This observation further proved the hypothesis that the behaviour of aptamers are affected significantly in the presence of AuNPs, which can impede their target binding abilities compared with free solution binding.

The findings in Chapter 3 hint at the potential disadvantages of selecting aptamers for small molecule targets using traditional bead immobilized affinity-based SELEX, which does not account for the downstream application of aptamers for detection in sensor platforms with various transduction modalities. This can be quite disadvantageous for the aptamer as shown in Chapter 3 because the SELEX process should have regarded the need for pre-conditioning aptamers to maintain the dynamic equilibrium between the free and adsorbed states that remain available to interact with the target. In the end the need for alternative SELEX strategies is highlighted.

In Chapter 4, the hypothesis that the SELEX method may have a significant impact on the way aptamers behave in subsequent downstream applications has been addressed. A new methamphetamine aptamer selected in solution, based on a novel AuNP SELEX procedure was tested. It was an achievement to find out that the new aptamer not only exhibited improved response towards meth, but also confirmed the previous hypothesis regarding the compromised equilibrium between the free and adsorbed states of aptamer in the presence of AuNPs. In short, 
the modified SELEX process (developed and implemented by collaborators) explicitly selected for aptamer sequences that maintained dynamic equilibrium and target-induced dissociation from AuNPs. The effect of the AuNP SELEX was evident when it was found that the response of the AuNP SELEX selected aptamers did not depend on the sequence of addition i.e. it was irrespective of dissociation vs association assay format. This suggested unconstrained ability of the aptamer in attaining target binding conformations when adhered to AuNPs unlike that observed in Chapter 3 i.e. the aptamer structures did not easily disintegrate on adsorption to AuNPs.

Having created new knowledge to develop meth sensors capable of detection below the legal threshold of $25 \mathrm{ng} / \mathrm{mL}$, albeit in spiked buffer solutions, Chapter 5 explores meth detection in oral fluid at biologically relevant concentrations. The challenge of application of AuNP aggregation assay in saliva was extensively discussed and demonstrated with UV-Vis and DLS studies. Subsequently, a point-of-care friendly saliva processing technique using syringe filters was successfully developed to address the challenge. In Chapter 5, the signals obtained in saliva were referenced against those in buffer in order to validate the salivary response. It was found that on an average, the signal response in saliva was suppressed 30-50\% depending on the saliva collection method used. As it was found that when the saliva was collected using a conventional saliva collection device, Quantisal, the recovery of meth in those samples were higher than when whole saliva was collected using passive drooling. Moreover, it was also established that around $90 \%$ of the meth in saliva can be recovered during syringe filtration assuring insignificant loss of meth during the filtration steps. Successful validation of the assay was demonstrated with blind sample trials that were spike-prepared by the collaborators, which was highly promising. Overall, the assay response was found to be inconsistent and irreproducible when the collection of saliva was not standardized. Despite all the practical challenges faced it was possible to achieve promising levels of detection first with the 34-mer dissociation assay $\sim 96 \mathrm{ng} / \mathrm{mL}$ and subsequently with the 42-mer, an improved LOD $35.8 \mathrm{ng} / \mathrm{mL}$ was achieved. Both the levels were although higher than the legally required cut off level of $25 \mathrm{ng} / \mathrm{mL}$, they were nonetheless very promising in highlighting the scope of these assays for further developments.

Finally, the need to develop a portable detection system to facilitate the application of the assay on-site was investigated in Chapter 6. A reliable, sensitive and portable light meter device integrated with an LED of $520 \mathrm{~nm}$ was designed. The cost-effective device, which cost $<\$ 100$, 
facilitated improved LOD achievement when compared to the standard UV-Vis plate reader. This was highly promising and may serve as a prototype for a more advanced future version. In conclusion, this thesis successfully achieved the specific objectives described in Chapter 1.

\section{Future perspectives}

As discovered in Chapter 3 aptamers selected via traditional assays get incapacitated on AuNPs surface, this knowledge was further used to develop aptamers using modified SELEX strategies which was also demonstrated in the thesis, but it must be noted that in spite of developing the aptamers using modified AuNP SELEX, the binding mechanism between aptamers and meth was similar to that for the traditional SELEX aptamer, the advantage was only in the attainment of equilibrium adsorption and desorption kinetics. Therefore, there is still immense scope for improvement in developing aptamers that undergo more prominent structural rearrangement leading to more specific target binding. This would enable the efficient utilization of these assays for the detection of SMs analytes. Aptamers that involve more hydrogen bond formation when undergoing a conformational change may be most suitable for the application in these assays.

ITC investigations are a work in progress, and it is a time consuming but important characterization technique for gaining more knowledge about the binding interactions between aptamers and analytes. Therefore, future investigations with various optimization parameters need to be tested to gain more insight and knowledge about the binding and how it can be improved to gain enhanced selection. Some reports about the possibility to change the binding mechanism by altering ionic conditions highlight the scope for improved binding characterization and optimization using ITC.

As investigated in Chapter 5, targeted saliva processing techniques proved most effective for mitigating protein effects in the matrix. It may be possible that further investigations into targeted electrolyte removal may be able to do away with the dilution. This may be possible with gel filtration techniques to desalt a sample, but it must be noted that this can also affect small molecules in the sample alike. Therefore, it may be challenging and requires further research. Electrophoresis may be a promising option for separating meth from proteins and other ions on the basis of charge and size before application to AuNPs assay. Dilution free techniques are especially preferred considering the challenge of detecting SMs in diluted sample given their low availability in 
undiluted biofluids to begin with. Strategies to remove electrolyte as well as proteins need to be investigated primarily to minimize dilution.

Lastly, Chapter 6 that demonstrates the successful validation of a light meter device against a standard UV-Vis spectrometer, can be further modified in terms of sample preparation methods for a complete on-site application. It may be possible to investigate microfluidic platforms that can be integrated with the device which would eliminate the use of pipettes and other lab-based consumables. Also, paper-based platforms for colorimetric assays may be an attractive alternative which offers disposable and flexible platform for colorimetric sensing and the visual response can be quantified using image analysis. 


\section{APPENDIX}

\section{Supplementary information relating to Chapter 3}

1) Colorimetric screening of meth binding aptamer candidates

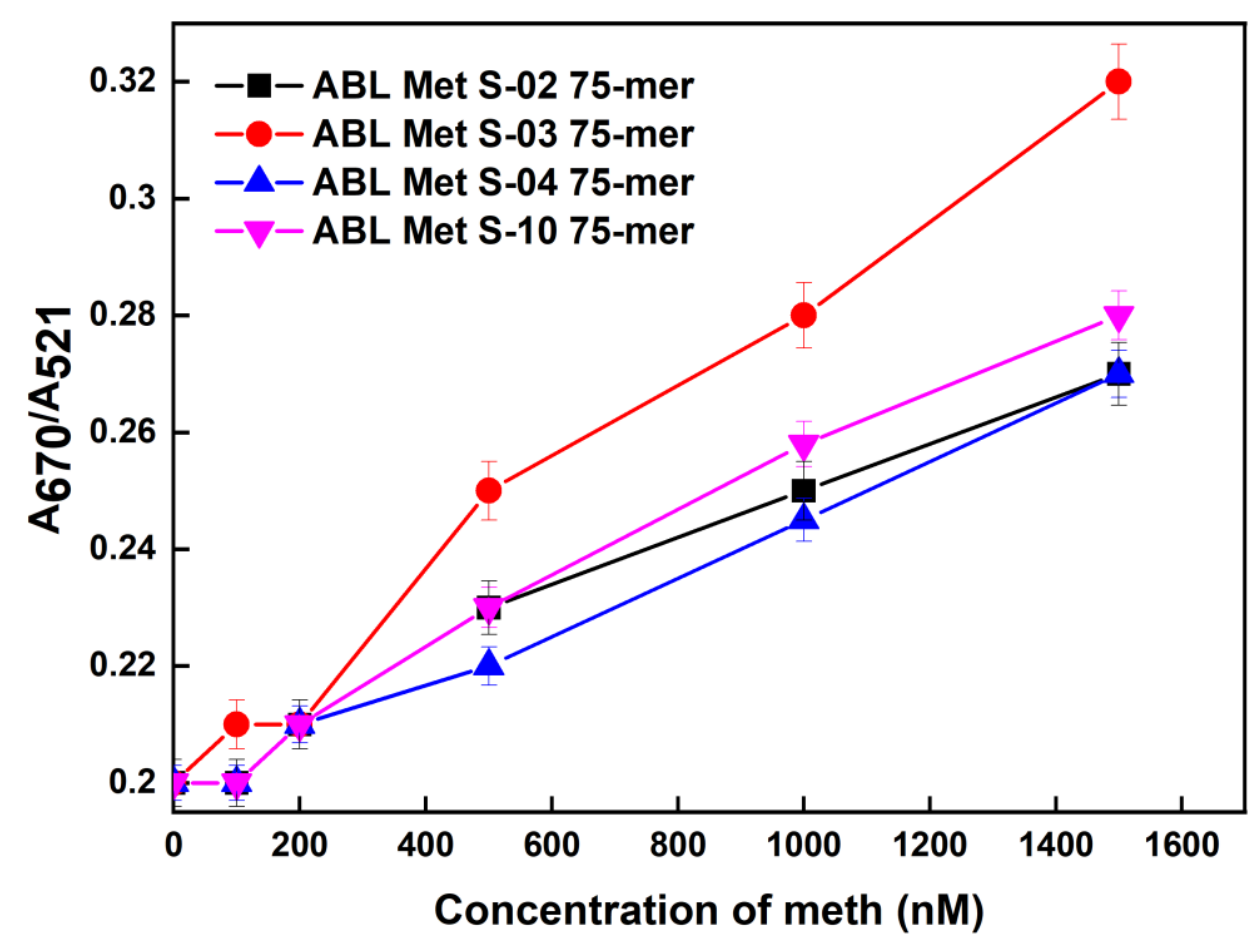

Figure A1 Colorimetric screening of a family of meth aptamers obtained from Auramer Bio. ABL Met S03 75-mer was the most promising among the other candidates. Error bars represent the mean of the standard deviation of three measurements. 


\section{2) UV-Vis and Dynamic Light Scattering (DLS) Size Characterization}
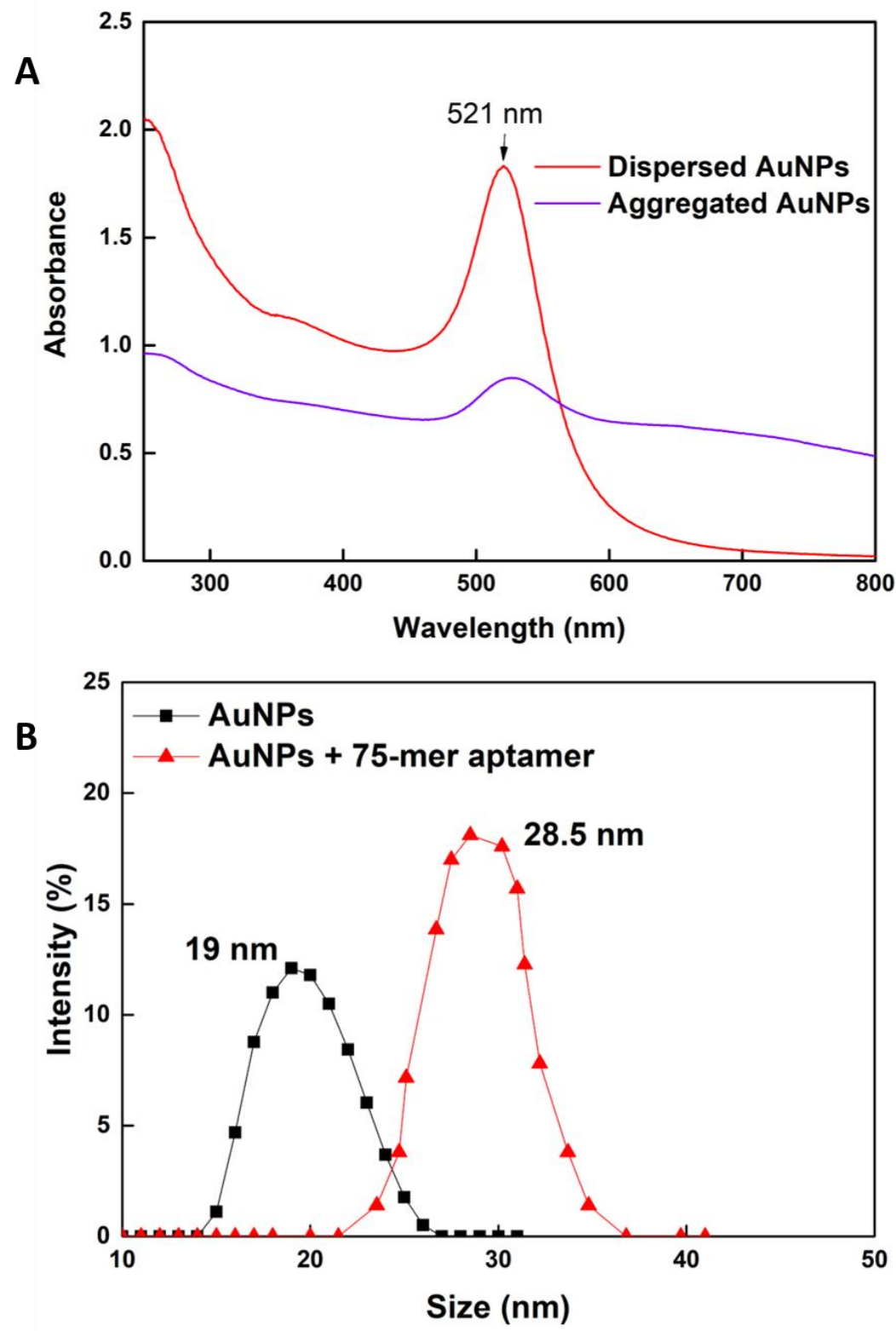

Figure A2 (A) UV-Vis characterization of AuNPs in dispersed and aggregated states. (B) DLS size characterization for AuNps and (AuNPs, 75-mer Aptamer) in aqueous media with a Poly dispersity index (Pdi) of 0.28 , viscosity $0.8872 \mathrm{cP}, \mathrm{RI} 1.33$ and z-average $19 \mathrm{~nm}$.

\section{3) pH, sensitivity, and salt optimization}

$\underline{\text { Sensitivity optimization }}$ 
A

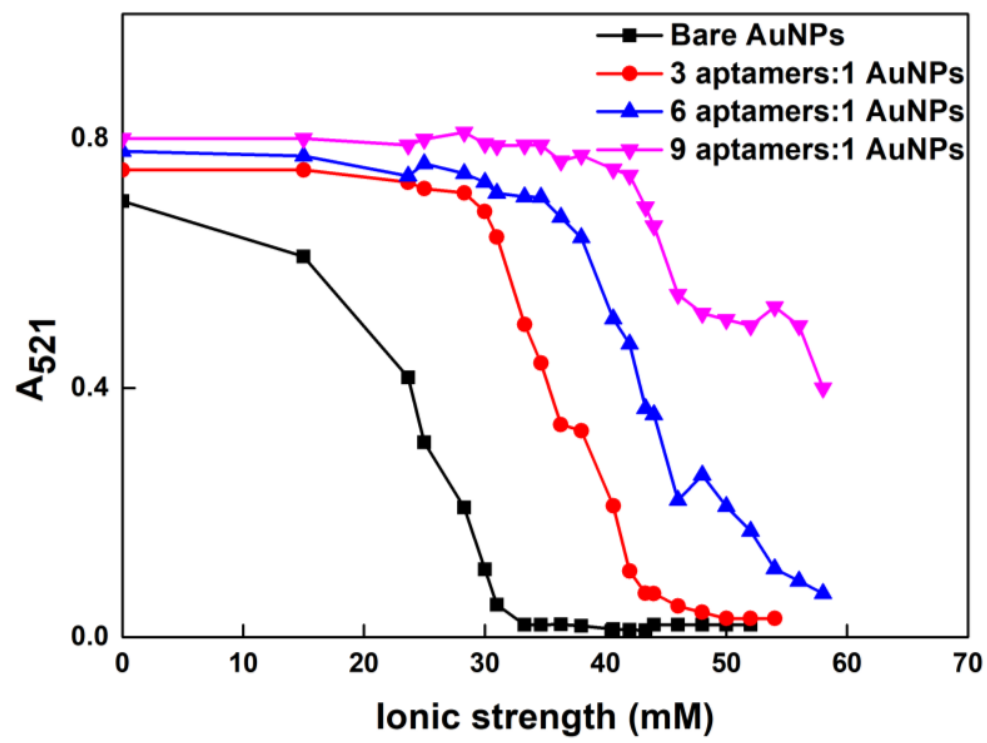

B

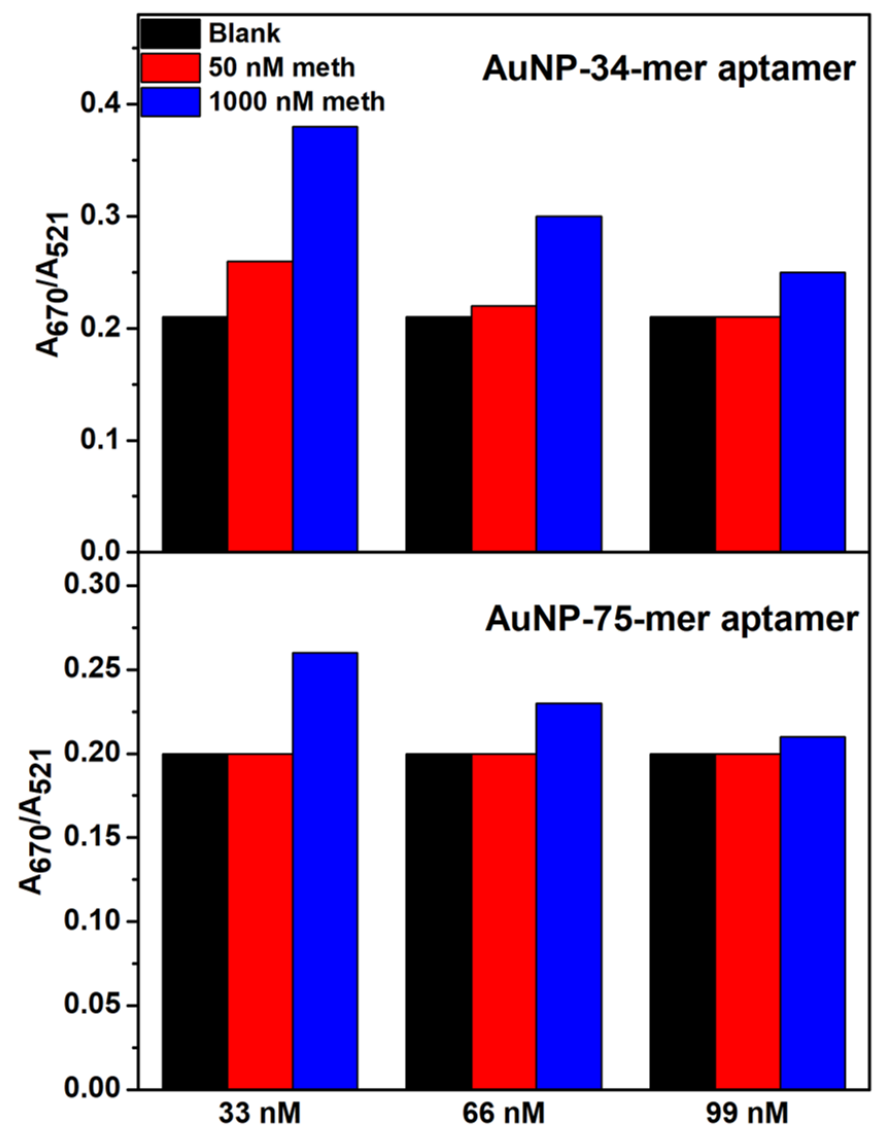

Figure A3 (A) Salt dependent aggregation for AuNPs mixed with increasing 75-mer aptamer coverage. (B) Sensitivity optimization of various concentrations of aptamer (33, 66 and $99 \mathrm{nM}$ ) such that the stoichiometric ratio of [Aptamer] : [AuNPs] are 3:1, 6:1 and 9:1 where [AuNPs] $=11 \mathrm{nM}$. 


\section{pH optimization}

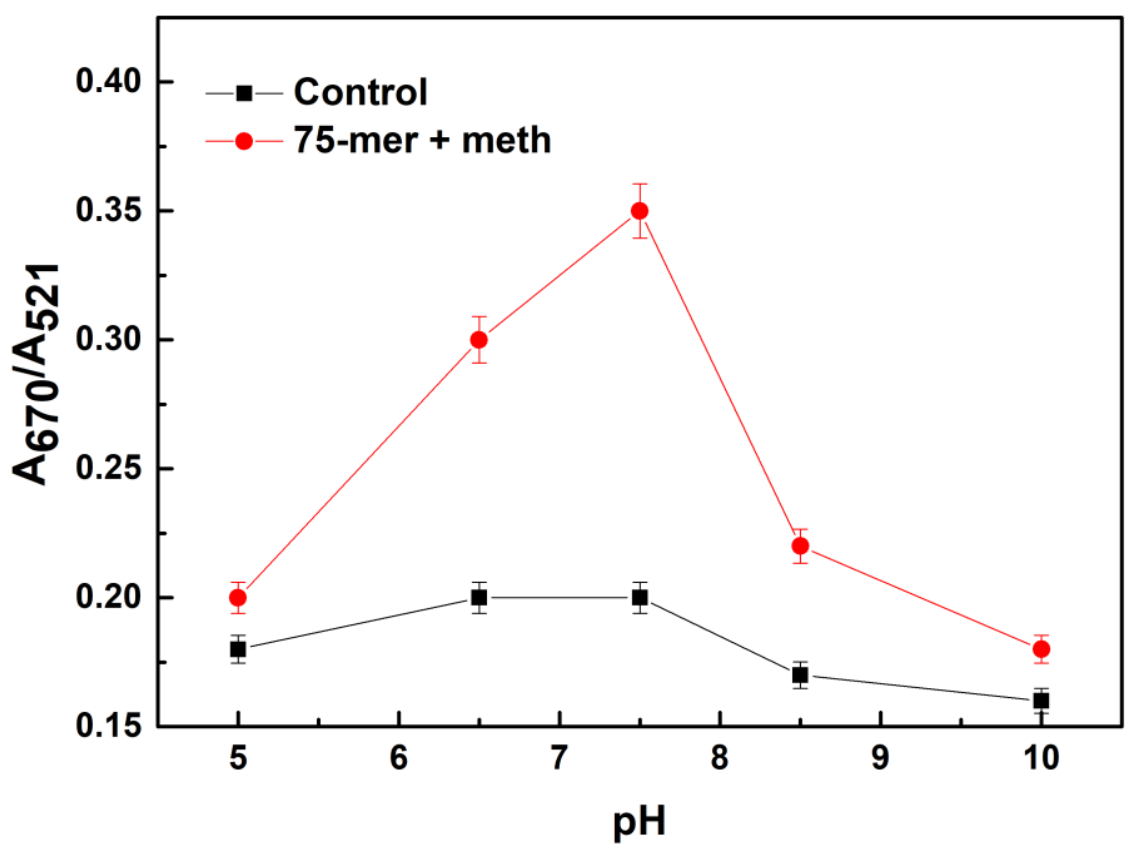

Figure A4 The response of the sensor at $\mathrm{pH} 5,6.5,7.5,8.5$ and 10. The concentration of meth was $1 \mu \mathrm{M}$. The best colorimetric response was obtained at $\mathrm{pH} 7.5$ and since the $\mathrm{pH}$ of oral fluid ranges between 6.2 7.6, $\mathrm{pH} 7.5$ was chosen as the optimal $\mathrm{pH}$. 
$\underline{\text { Salt concentration optimisation }}$
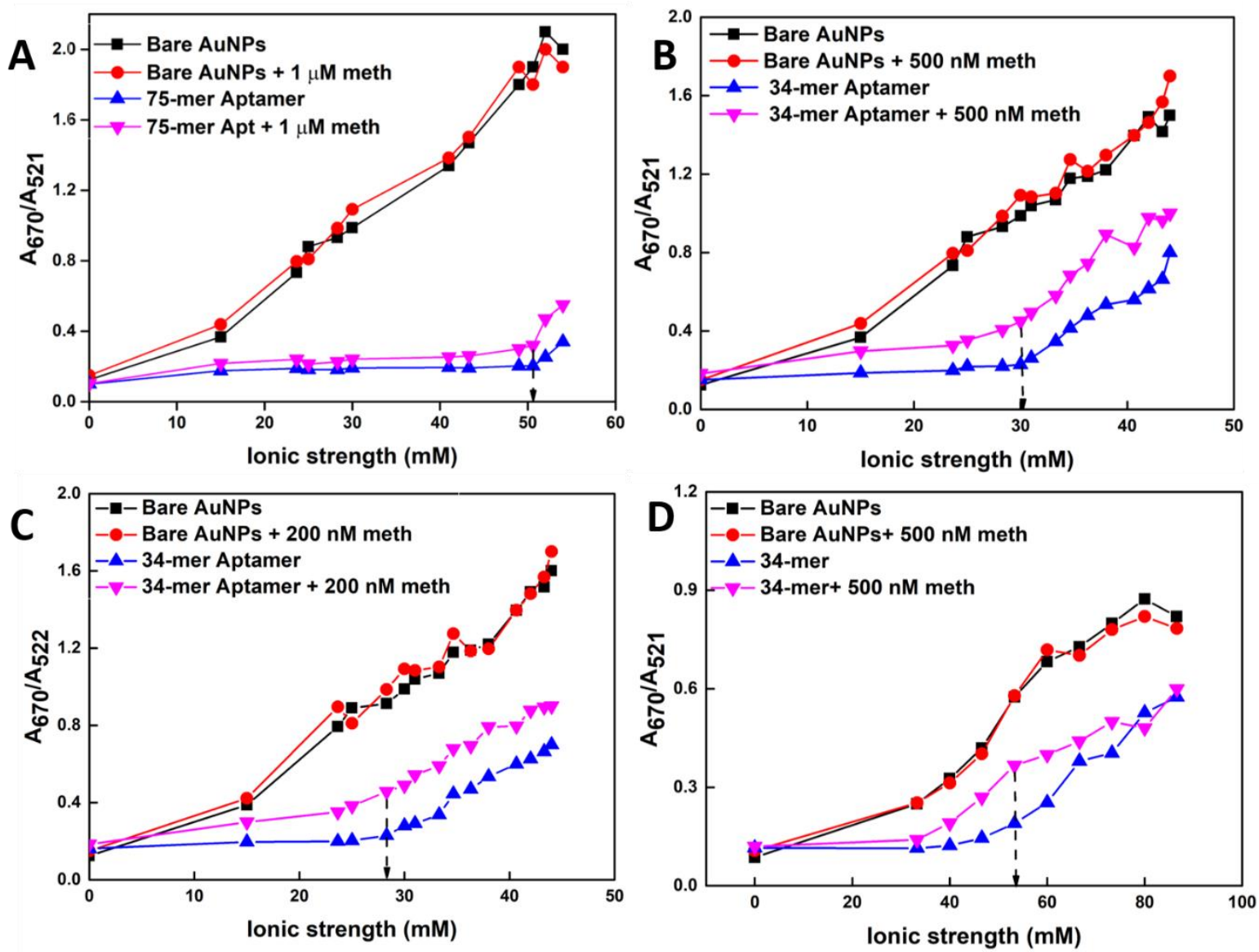

Figure A5 Salt optimization graph for A) 75-mer aptamer following aptamer dissociation assay. The optimal ionic strength $(51 \mathrm{mM})$ is indicated by the arrow. B) 34-mer aptamer following aptamer dissociation assay. The optimal ionic strength $(30 \mathrm{mM})$ is indicated by the arrow. C) 34-mer following aptamer association assay. The optimal ionicstrength $(28 \mathrm{mM})$ is indicated by the arrow. Inset image illustrates the visually detectable state at the optimal ionic strength. D) 34-mer aptamer in oral fluid. The optimal ionic strength $(53.6 \mathrm{mM})$ is indicated by the arrow. 


\section{4) Linear fit for 75-mer and 34-mer aptamers using dissociation assay}
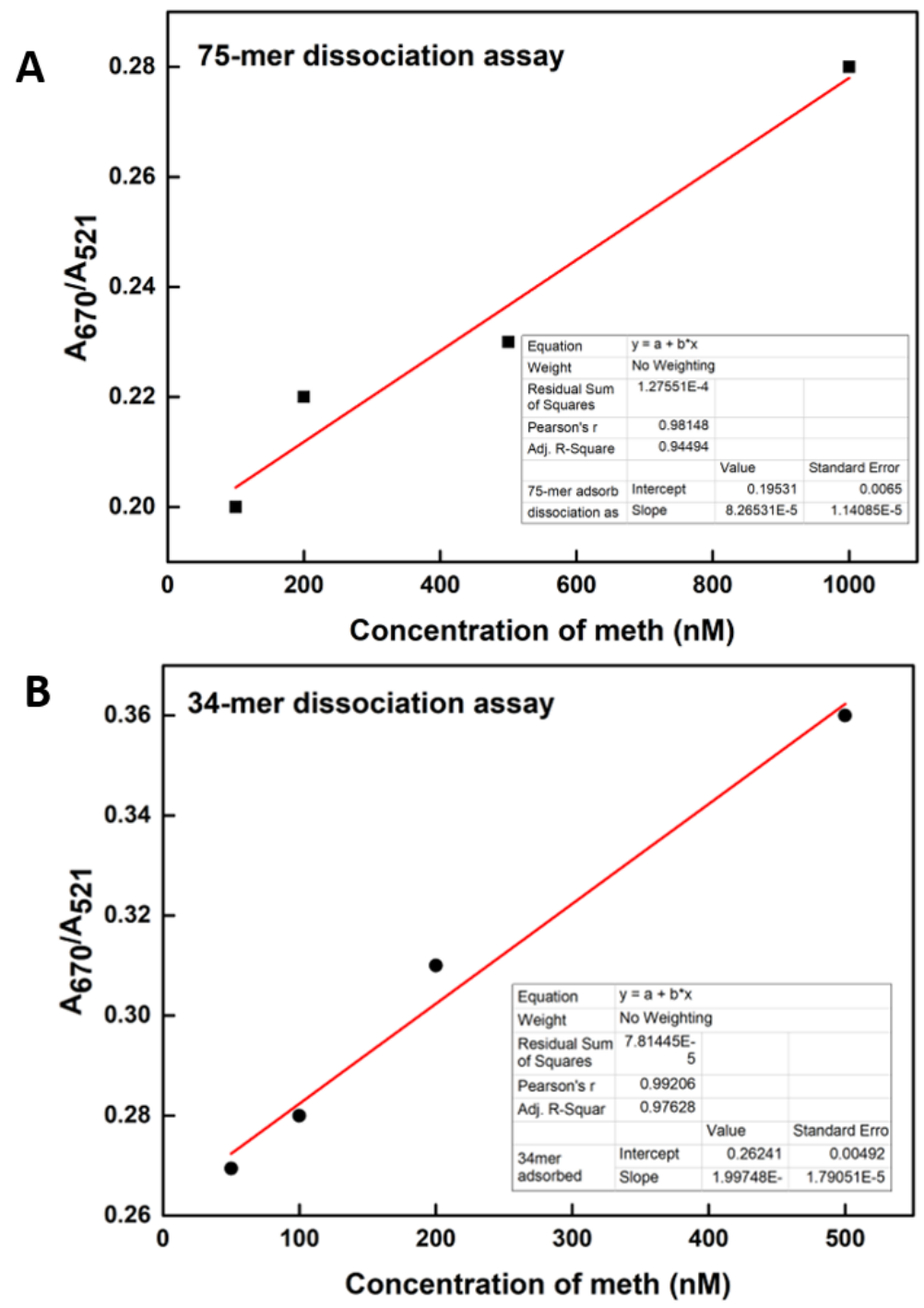

Figure A6 Linear fit curves of A) 75-mer and B) 34-mer. 


\section{5) Control studies to rule out non-specific binding}

\section{Evaluating the effect of meth on aptamer adsorption}

Optimal salt concentration for the AuNPs + 34-mer aptamer samples was found to be $30 \mathrm{mM}$. This was considered as the reference sample. AuNPs + meth was incubated for 30 minutes and no destabilizing effect of met hon AuNPs was observed at $500 \mathrm{nM}$ meth. Next, 34-mer aptamer was added to the sample and incubated for another $30 \mathrm{mins}$, the same salt concentration $(30 \mathrm{mM})$ was added to see if the sample remained stable or aggregated. It was found that the sample remained stable suggesting successful adsorption of aptamer in the presence of $500 \mathrm{nM}$ meth and no inhibition effect of meth towards aptamer adsorption. Then, AuNPs +34-mer aptamer followed by meth was added and after addition of salt the samples aggregated like expected suggesting target induced aptamer desorption. Thus, ruling out any significant effect of meth on aptamer adsorption at $500 \mathrm{nM}$.

Same steps were repeated for a higher meth concentration, $50 \mu \mathrm{M}$ and an onset of aggregation in AuNPs $+50 \mu \mathrm{M}$ samples were observed. This clarified that meth above $20 \mu \mathrm{M}$ was unsuitable for the colorimetric sensing assay. The observation was not surprising owing to the likelihood of an interaction between first, a primary amine containing molecule and secondly, the positive charge of meth made it unsuitable for application at higher concentrations (above $20 \mu \mathrm{M}$ ). 


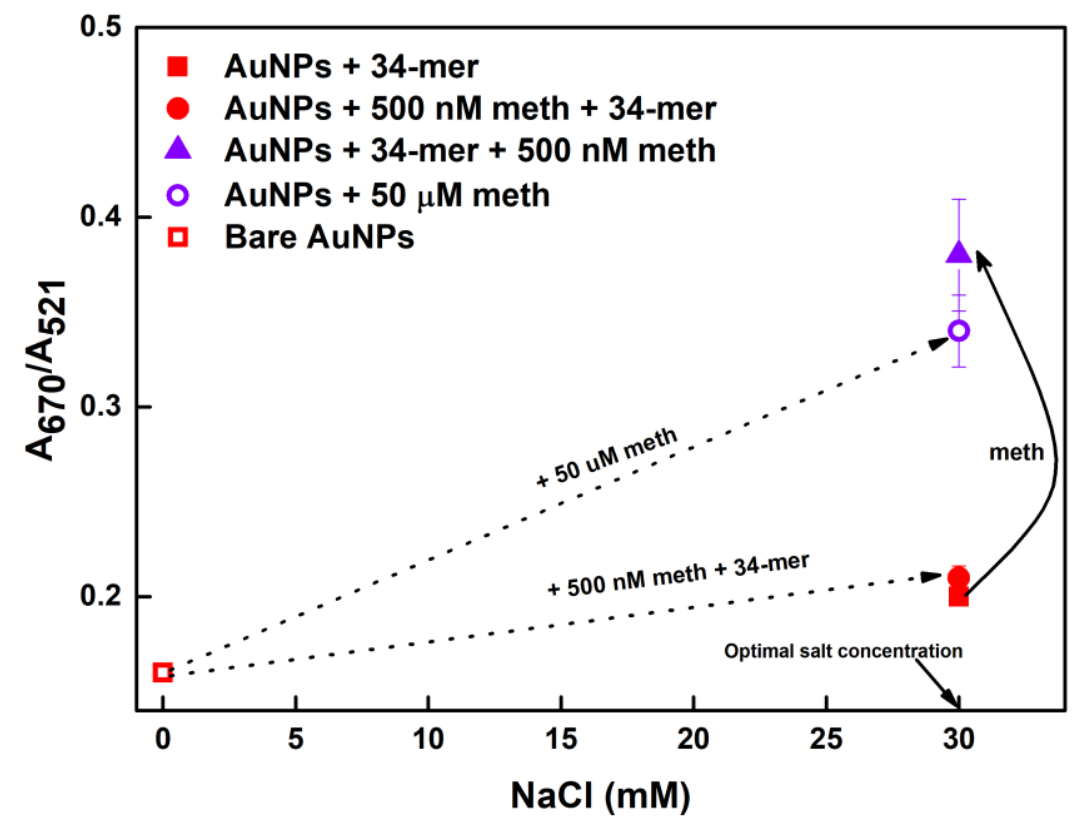

Figure A7 Control studies to evaluate the effect of meth on the adsorption of aptamer on AuNPs. It was found that meth at nanomolar concentrations tested in the present study showed no interfering abilities and at higher than $20 \mu \mathrm{M}$ meth destabilized AuNPs. 


\section{Control with bare AuNPs + interfering molecules}

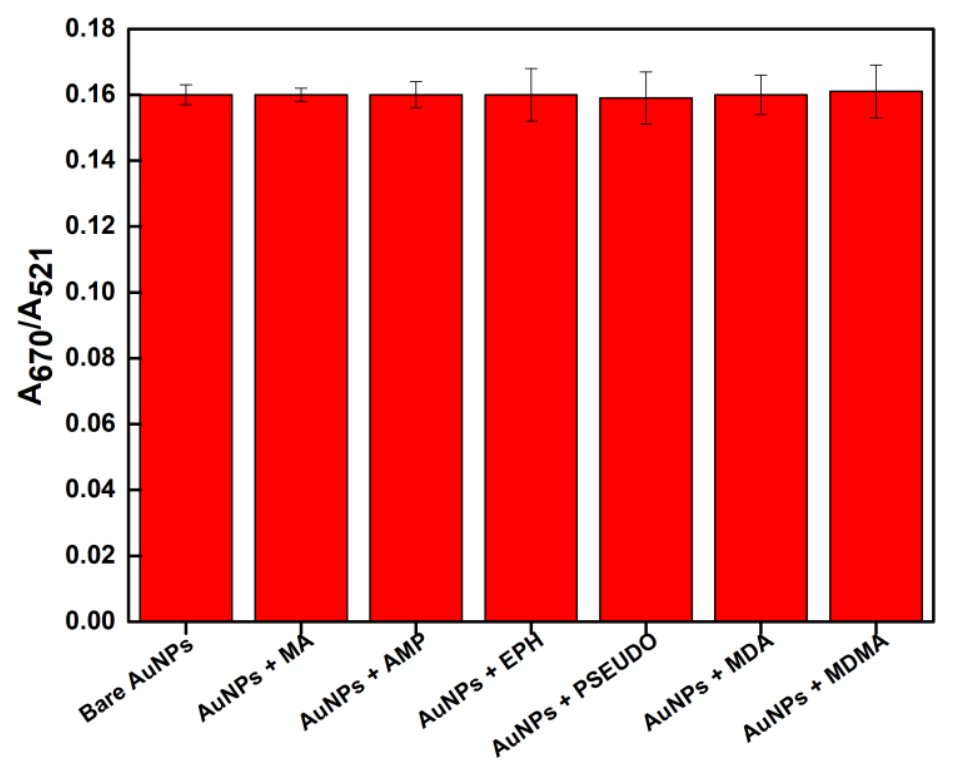

Figure A8 Bare AuNPs control for the different interfering molecules at $200 \mathrm{nM}$. The control showed no effect on stability of the AuNPs.

\section{6) Fluorescence assay reveals binding affinity $\sim$ independent binding technique}

This assay is based on the ability of the SG fluorescence dye to intercalate with partially doublestranded DNA (pdsDNA). A fluorescence emission only occurs when SG is intercalated into pdsDNA; when the SG is displaced from the DNA strand the fluorescence emission ceases.

Five different samples of the parent 75-mer aptamer-SG complex at a given concentration with increasing Meth concentrations were monitored by fluorescence spectroscopy. A proportional decrease of fluorescence intensity at $520 \mathrm{~nm}$, with increasing meth concentration, is monitored. This fluorescence decrease is produced by the competition between the meth and the SG molecule to interact with the DNA strand or it is produced by the release of the SG molecule due to an aptamer structural change. 

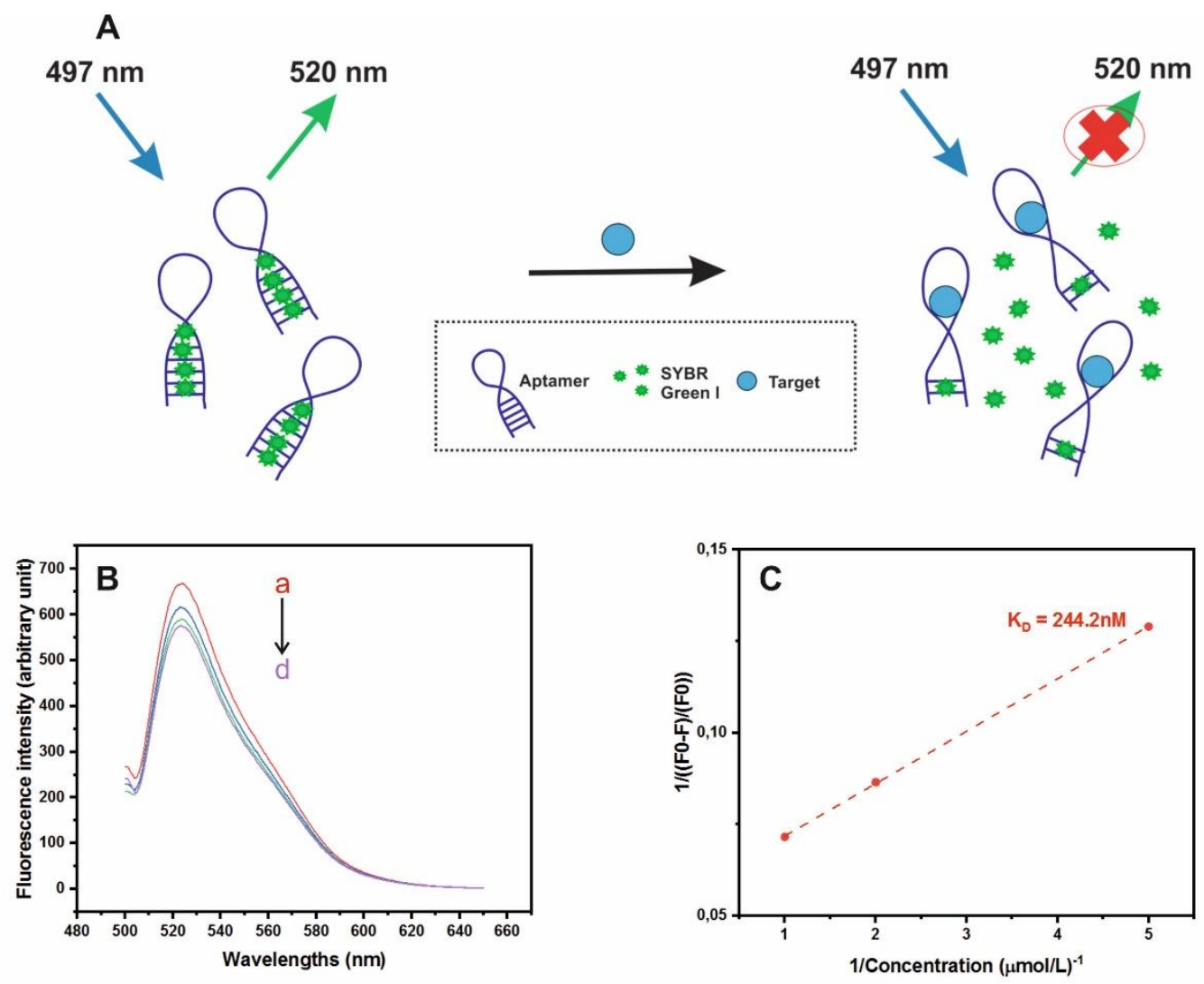

Figure A9. SG assay with a methamphetamine titration $(0 \mu \mathrm{M}$ (a), $0.2 \mu \mathrm{M}$ (b), $0.5 \mu \mathrm{M}$ (c) and $1 \mu \mathrm{M}$ (d)) for the parent 75-mer aptamer. (A) General schematic of the SG assay (B) A fluorescence decrease at 520 $\mathrm{nm}$ is monitored. (C) Linearization of the Langmuir isotherm.

The normalized fluorescence raw data plotted in function of the meth concentration gives the known Langmuir behavior. Therefore, a Langmuir isotherm has been drawn for the parent 75-mer aptamer. The equation of the Langmuir isotherm, related to a fluorescence experiment where the $[$ Meth] is greater than the [Aptamer], is the following:

$$
\frac{F_{0}-F}{F_{0}}=B \max \times \frac{[\text { Meth }]}{[\text { Meth }]+K_{D}}
$$

where, $F_{0}$ is the initial fluorescence of the Aptamer-SG complex without Meth, $F$ represents the fluorescence of the Aptamer-SG-Meth complex at one meth concentration, Bmax is the isotherm constant, [Meth] represents the concentration of Meth and $K_{D}$ is the dissociation constant. Based on the linearization of equation (1), $K_{D}$ values was calculated. 
The above described procedure was used to similarly extract the Kd for 34-mer meth binding aptamer and a $\mathrm{Kd}$ of $133 \pm 15 \mathrm{nM}$ was obtained. A negative control with a 40-mer mutated aptamer was also done following the same procedure and no fluorescence signal drop was observed with increasing concentration of meth, confirming that the stem and the resulting loop region is essential for binding.
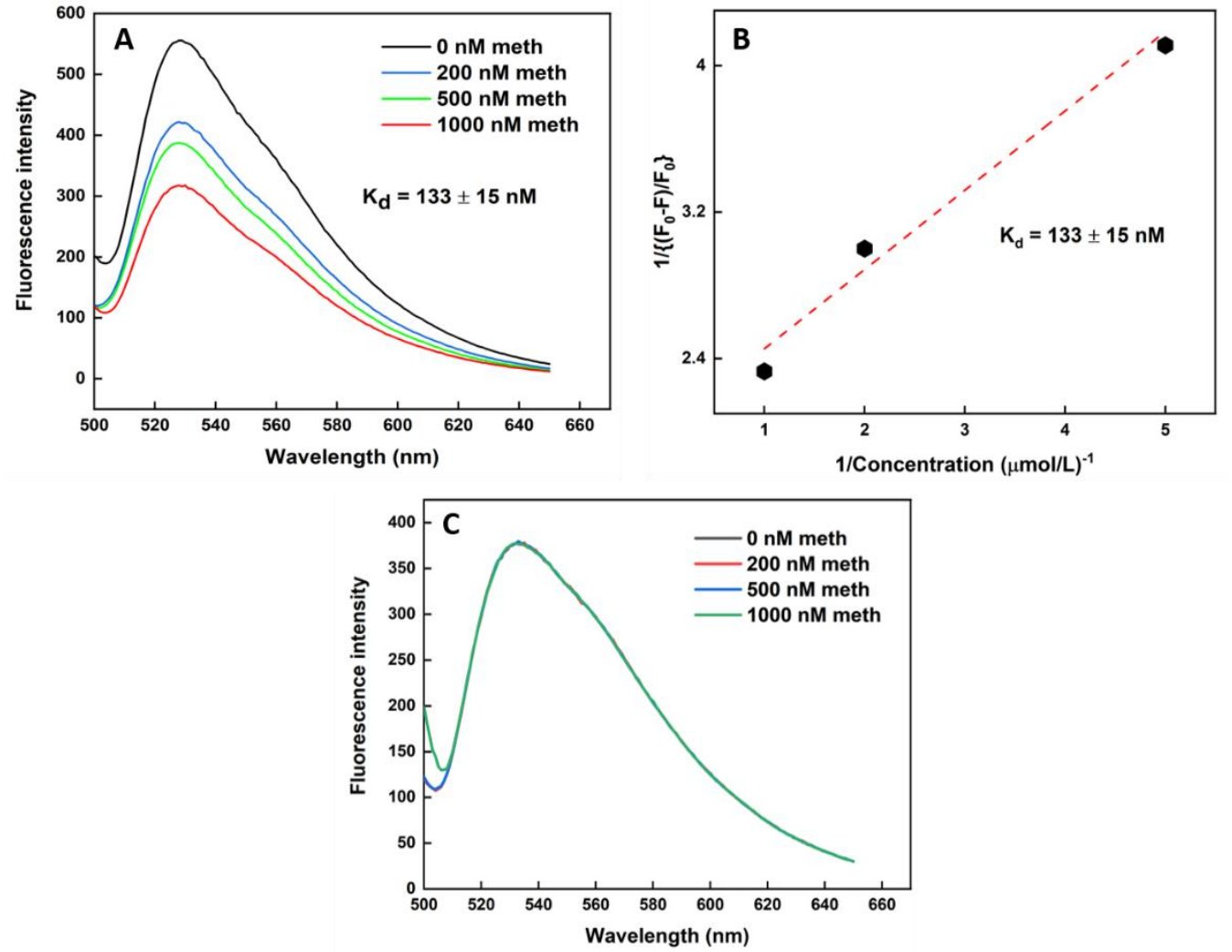

Figure A10. SG assay with a methamphetamine titration $(0,0.2,0.5$ and $1 \mu \mathrm{M})$ for the 34-mer aptamer and a mutant 40-mer aptamer. (A) A fluorescence decrease at $520 \mathrm{~nm}$ is monitored for the 34-mer aptamer. (B) Linearization of the Langmuir isotherm. (C) No fluorescence decrease at $520 \mathrm{~nm}$ is observed for the mutant 40 -mer. 


\section{7) Zeta-potential table of values}

Table A1. Zeta-potential values of 34-mer meth binding aptamer and 34-mer random aptamer with and without meth. \pm STD, $n=3$. pH 7.5, viscosity of 0.8872 cP, R.I 1.33

\begin{tabular}{|c|c|c|c|}
\hline Sample & $\begin{array}{ll}\text { Incubation } & \text { Time } \\
\text { (minutes) } & \end{array}$ & $\begin{array}{l}\text { Zeta-potential of } \\
\text { AuNPs, Aptamer } \\
(\mathrm{mV})\end{array}$ & $\begin{array}{l}\text { Zeta-potential of } \\
(\text { AuNPs,Aptamer)+meth } \\
(\mathrm{mV})\end{array}$ \\
\hline 34-mer & 30 & $-33.4 \pm 0.18$ & $-29 \pm 0.65$ \\
\hline Random 34-mer & & $-42.3 \pm 0.47$ & $-41 \pm 0.81$ \\
\hline 34-mer & & $-38 \pm 0.81$ & $-36.3 \pm 0.92$ \\
\hline Random 34-mer & & $-42.2 \pm 0.61$ & $-41.3 \pm 0.5$ \\
\hline 34-mer & 90 & $-41.7 \pm 1.35$ & $-40 \pm 0.8$ \\
\hline Random 34-mer & & $-43 \pm 0.74$ & $-42.3 \pm 0.95$ \\
\hline
\end{tabular}




\section{8) Random sequence m-fold predicted structure}

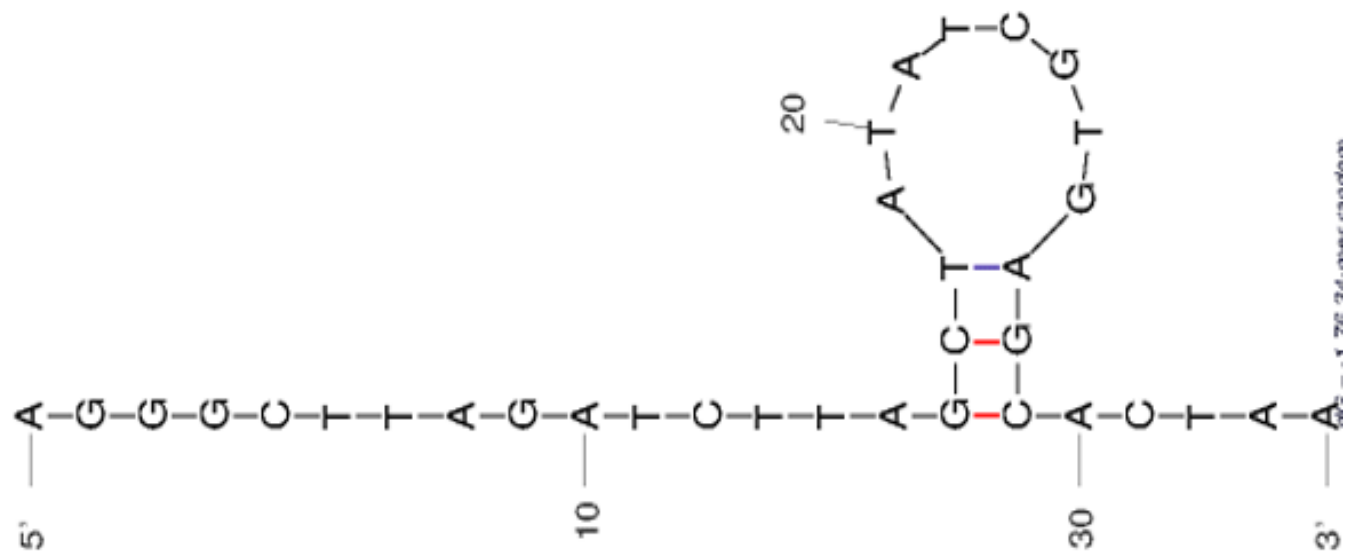

Figure A11 Predicted M-fold 2-D structure of 34-mer random sequence 


\section{9) Comparison of responses from 75-mer and 34-mer in dissociation and association assays}

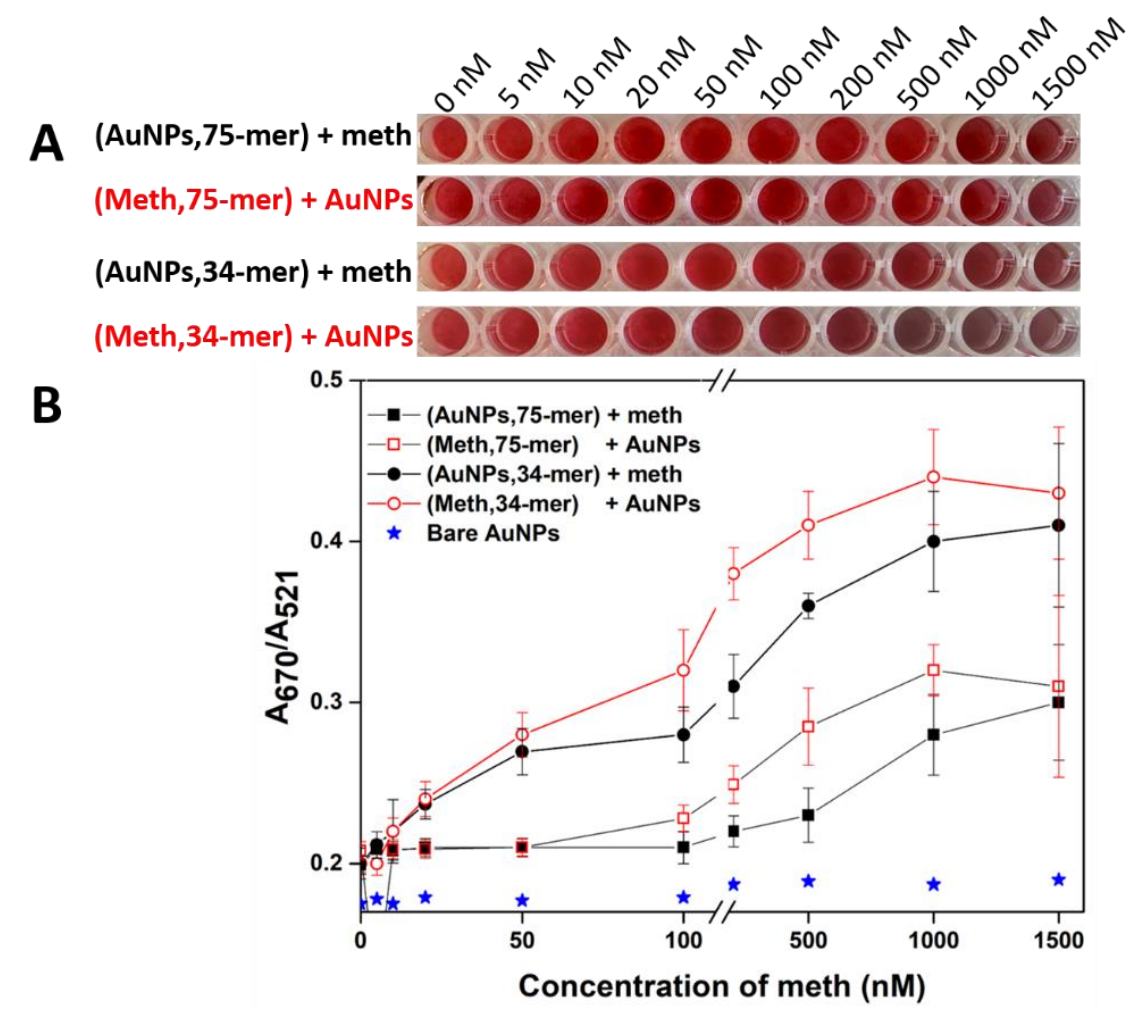

Figure A12 (A) Illustration of colour changes occurring across samples over a range of meth concentrations from 0 - $1500 \mathrm{nM}$. (B) Aggregation plot showing ratio of absorbances at 670/521 vs concentration of meth $(\mathrm{nM})$ to compare the sensing responses between parent 75-mer and truncated 34-mer using the dissociation and aptamer association assay responses. 
10) Response of 34-mer in oral fluid using aptamer dissociation and association assay

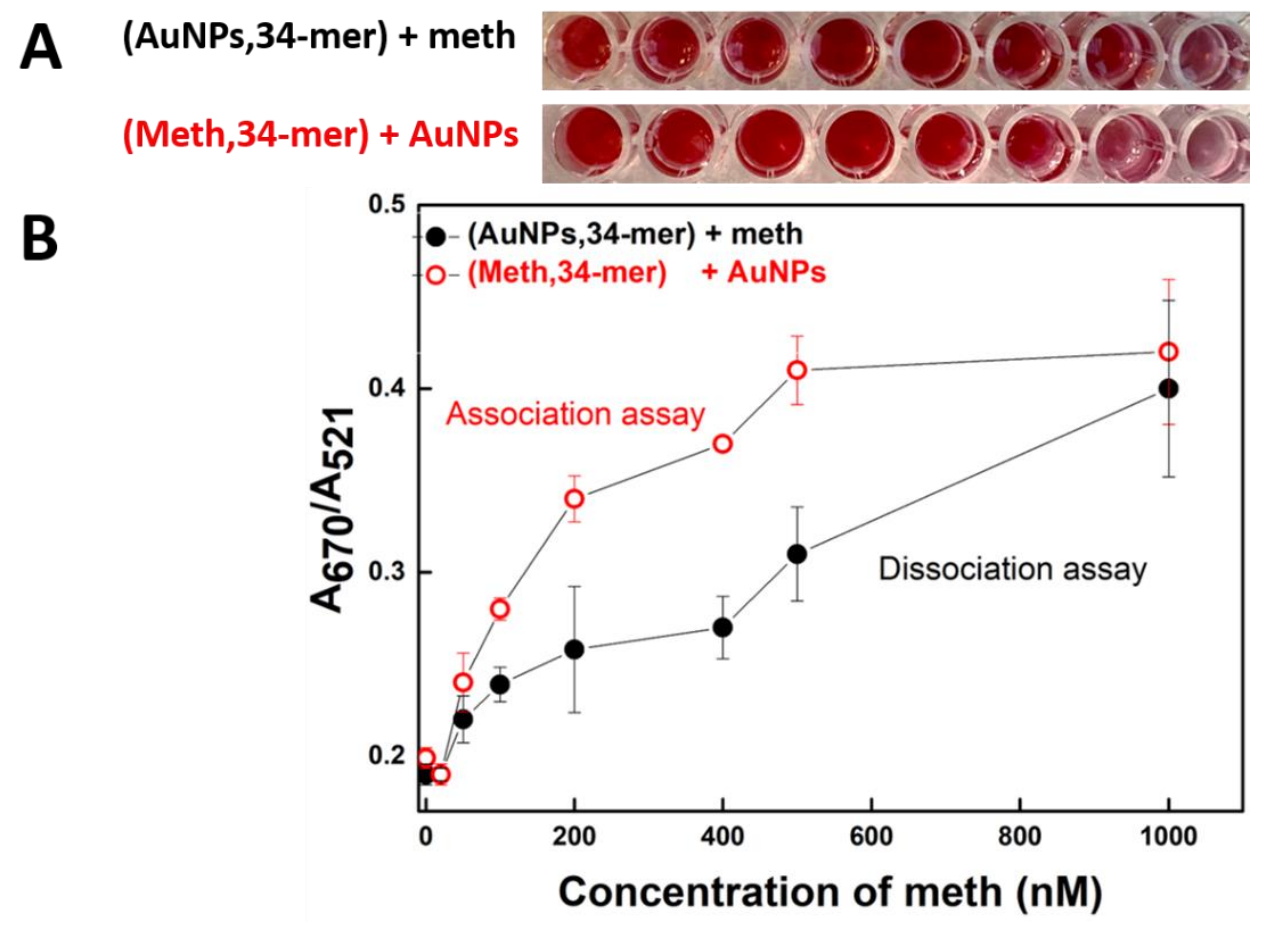

Figure A13 Comparison of colorimetric responses from the aptamer association and aptamer dissociation assay formats in oral fluid. 


\section{REFERENCES}

(1) Anglin, M. D.; Burke, C.; Perrochet, B.; Stamper, E.; Dawud-Noursi, S. History of the Methamphetamine Problem. J. Psychoactive Drugs 2000, 32 (2), 137-141.

(2) Parsons NL. Meth mania: A history of methamphetamine. Boulder, CO: Lynne Rienner Publishers, Incorporated; 2014.

(3) Matsumoto T, Kamijo A, Miyakawa T, Endo K, Yabana T, Kishimoto H, Okudaira K, Iseki E, Sakai T, Kosaka K. Methamphetamine in Japan: the consequences of methamphetamine abuse as a function of route of administration. Addiction. 2002;97(7):80917.

(4) Maxwell, J. C.; Brecht, M.-L. Methamphetamine: Here We Go Again? Addict. Behav. 2011, 36 (12), 1168-1173. https://doi.org/10.1016/j.addbeh.2011.07.017.

(5) Karila, L.; Weinstein, A.; Aubin, H.-J.; Benyamina, A.; Reynaud, M.; Batki, S. L. Pharmacological Approaches to Methamphetamine Dependence: A Focused Review. Br. J. Clin. Pharmacol. 2010, 69 (6), 578-592. https://doi.org/10.1111/j.1365-2125.2010.03639.x.

(6) Albertson, T. E.; Derlet, R. W.; Van Hoozen, B. E. Methamphetamine and the Expanding Complications of Amphetamines. West. J. Med. 1999, 170 (4), 214-219.

(7) Brinded, P. M.; Simpson, A. I.; Laidlaw, T. M.; Fairley, N.; Malcolm, F. Prevalence of Psychiatric Disorders in New Zealand Prisons: A National Study. Aust. N. Z. J. Psychiatry 2001, 35 (2), 166173. https://doi.org/10.1046/j.1440-1614.2001.00885.x.

(8) HUESTIS, M. A.; CONE, E. J. Methamphetamine Disposition in Oral Fluid, Plasma, and Urine. Ann. N. Y. Acad. Sci. 2007, 1098, 104-121. https://doi.org/10.1196/annals.1384.038.

(9) Kish, S. J. Pharmacologic Mechanisms of Crystal Meth. CMAJ Can. Med. Assoc. J. 2008, 178 (13), 1679-1682. https://doi.org/10.1503/cmaj.071675.

(10) Wang, G.-J.; Volkow, N. D.; Chang, L.; Miller, E.; Sedler, M.; Hitzemann, R.; Zhu, W.; Logan, J.; Ma, Y.; Fowler, J. S. Partial Recovery of Brain Metabolism in Methamphetamine Abusers After Protracted Abstinence. Am. J. Psychiatry 2004, 161 (2), 242-248. https://doi.org/10.1176/appi.ajp.161.2.242.

(11) Crouch, D. J.; Day, J.; Baudys, J.; Fatah, A. A. Evaluation of Saliva/Oral Fluid as an Alternate Drug Testing Specimen. 77.

(12) Cone, E. J. Saliva Testing for Drugs of Abuse. Ann. N. Y. Acad. Sci. 1993, 694, 91-127. https://doi.org/10.1111/j.1749-6632.1993.tb18346.x.

(13) Dawes, C. Considerations in the Development of Diagnostic Tests on Saliva. Ann. N. Y. Acad. Sci. 1993, 694, 265-269. https://doi.org/10.1111/j.1749-6632.1993.tb18359.x.

(14) Cone, E. J.; Kumor, K.; Thompson, L. K.; Sherer, M. Correlation of Saliva Cocaine Levels with Plasma Levels and with Pharmacologic Effects after Intravenous Cocaine Administration in Human Subjects. J. Anal. Toxicol. 1988, 12 (4), 200-206. https://doi.org/10.1093/jat/12.4.200.

(15) Kinetics, salivary excretion of amphetamine isomers, and effect of urinary pH - Wan - 1978 Clinical Pharmacology \&amp; Therapeutics - Wiley Online Library https://ascpt.onlinelibrary.wiley.com/doi/abs/10.1002/cpt1978235585 (accessed 2021 -09-27).

(16) Bellagambi, F. G.; Lomonaco, T.; Salvo, P.; Vivaldi, F.; Hangouët, M.; Ghimenti, S.; Biagini, D.; Di Francesco, F.; Fuoco, R.; Errachid, A. Saliva Sampling: Methods and Devices. An Overview. TrAC Trends Anal. Chem. 2020, 124, 115781. https://doi.org/10.1016/j.trac.2019.115781.

(17) Cheng, W.-C.; Mok, V. K.-K.; Chan, K.-K.; Li, A. F.-M. A Rapid and Convenient LC/MS Method for Routine Identification of Methamphetamine/Dimethylamphetamine and Their Metabolites in Urine. Forensic Sci. Int. 2007, 166 (1), 1-7. 
(18) Kuroda, N.; Nomura, R.; al-Dirbashi, O.; Akiyama, S.; Nakashima, K. Determination of Methamphetamine and Related Compounds by Capillary Electrophoresis with UV and LaserInduced Fluorescence Detection. J. Chromatogr. A 1998, 798 (1-2), 325-334.

(19) Ruangyuttikarn, W.; Moody, D. E. Comparison of Three Commercial Amphetamine Immunoassays for Detection of Methamphetamine, Methylenedioxyamphetamine, Methylenedioxymethamphetamine, and Methylenedioxyethylamphetamine. J. Anal. Toxicol. 1988, 12 (4), 229-233. https://doi.org/10.1093/jat/12.4.229.

(20) Nieddu, M.; Burrai, L.; Baralla, E.; Pasciu, V.; Varoni, M. V.; Briguglio, I.; Demontis, M. P.; Boatto, G. ELISA Detection of 30 New Amphetamine Designer Drugs in Whole Blood, Urine and Oral Fluid Using Neogen ${ }^{\circledR}$ "Amphetamine" and "Methamphetamine/MDMA" Kits. J. Anal. Toxicol. 2016, 40 (7), 492-497. https://doi.org/10.1093/jat/bkw060.

(21) Oiestad, E. L.; Johansen, U.; Christophersen, A. S. Drug Screening of Preserved Oral Fluid by Liquid Chromatography-Tandem Mass Spectrometry. Clin. Chem. 2006, 53 (2), 300-309. https://doi.org/10.1373/clinchem.2006.074237.

(22) Pujadas, M.; Pichini, S.; Civit, E.; Santamariña, E.; Perez, K.; de la Torre, R. A Simple and Reliable Procedure for the Determination of Psychoactive Drugs in Oral Fluid by Gas ChromatographyMass Spectrometry. J. Pharm. Biomed. Anal. 2007, 44 (2), 594-601. https://doi.org/10.1016/j.jpba.2007.02.022.

(23) Ebrahimi, M.; Johari-Ahar, M.; Hamzeiy, H.; Barar, J.; Mashinchian, O.; Omidi, Y. Electrochemical Impedance Spectroscopic Sensing of Methamphetamine by a Specific Aptamer. Biolmpacts BI 2012, 2 (2), 91-95. https://doi.org/10.5681/bi.2012.013.

(24) Nieddu, M.; Burrai, L.; Baralla, E.; Pasciu, V.; Varoni, M. V.; Briguglio, I.; Demontis, M. P.; Boatto, G. ELISA Detection of 30 New Amphetamine Designer Drugs in Whole Blood, Urine and Oral Fluid Using Neogen ${ }^{\circledR}$ "Amphetamine" and "Methamphetamine/MDMA" Kits. J. Anal. Toxicol. 2016, 40 (7), 492-497. https://doi.org/10.1093/jat/bkw060.

(25) Pujadas, M.; Pichini, S.; Civit, E.; Santamariña, E.; Perez, K.; de la Torre, R. A Simple and Reliable Procedure for the Determination of Psychoactive Drugs in Oral Fluid by Gas ChromatographyMass Spectrometry. J. Pharm. Biomed. Anal. 2007, 44 (2), 594-601. https://doi.org/10.1016/j.jpba.2007.02.022.

(26) Oiestad, E. L.; Johansen, U.; Christophersen, A. S. Drug Screening of Preserved Oral Fluid by Liquid Chromatography-Tandem Mass Spectrometry. Clin. Chem. 2006, 53 (2), 300-309. https://doi.org/10.1373/clinchem.2006.074237.

(27) Vindenes, V.; Yttredal, B.; Øiestad, E. L.; Waal, H.; Bernard, J. P.; Mørland, J. G.; Christophersen, A. S. Oral Fluid Is a Viable Alternative for Monitoring Drug Abuse: Detection of Drugs in Oral Fluid by Liquid Chromatography-Tandem Mass Spectrometry and Comparison to the Results from Urine Samples from Patients Treated with Methadone or Buprenorphine. J. Anal. Toxicol. 2011, 35 (1), 32-39. https://doi.org/10.1093/anatox/35.1.32.

(28) Perkins, M.; Langford, V. Application of Routine Analysis Procedures to a Direct Mass Spectrometry Technique: Selected Ion Flow Tube Mass Spectrometry (SIFT-MS). Rev. Sep. Sci. 2021, 3 (1), e21003-e21003. https://doi.org/10.17145/rss.21.003.

(29) Asbridge, M.; Ogilvie, R. A Feasibility Study of Roadside Oral Fluid Drug Testing. 21.

(30) Concheiro, M.; de Castro, A.; Quintela, Ó.; Cruz, A.; López-Rivadulla, M. Confirmation by LC-MS of Drugs in Oral Fluid Obtained from Roadside Testing. Forensic Sci. Int. 2007, 170 (2), 156-162. https://doi.org/10.1016/j.forsciint.2007.03.034.

(31) Sun J, Xianyu Y, Jiang X. Point-of-care biochemical assays using gold nanoparticle-implemented microfluidics. Chemical Society Reviews. 2014;43(17):623953.

(32) Bhalla, N.; Jolly, P.; Formisano, N.; Estrela, P. Introduction to Biosensors. Essays Biochem. 2016, 60 (1), 1-8. 
(33) Zahra, Q. ul A.; Luo, Z.; Ali, R.; Khan, M. I.; Li, F.; Qiu, B. Advances in Gold Nanoparticles-Based Colorimetric Aptasensors for the Detection of Antibiotics: An Overview of the Past Decade. Nanomaterials 2021, 11 (4), 840. https://doi.org/10.3390/nano11040840.

(34) Mungroo, N. A.; Neethirajan, S. Biosensors for the Detection of Antibiotics in Poultry Industry-A Review. Biosensors 2014, 4 (4), 472-493. https://doi.org/10.3390/bios4040472.

(35) Wang, Q.; Yang, Q.; Wu, W. Progress on Structured Biosensors for Monitoring Aflatoxin B1 From Biofilms: A Review. Front. Microbiol. 2020, 11, 408. https://doi.org/10.3389/fmicb.2020.00408.

(36) Kawamura, A.; Miyata, T. 4.2 - Biosensors. In Biomaterials Nanoarchitectonics; Ebara, M., Ed.; William Andrew Publishing, 2016; pp 157-176. https://doi.org/10.1016/B978-0-323-371278.00010-8.

(37) Mehrotra, P. Biosensors and Their Applications - A Review. J. Oral Biol. Craniofacial Res. 2016, 6 (2), 153-159. https://doi.org/10.1016/j.jobcr.2015.12.002.

(38) Damborský, P.; Švitel, J.; Katrlík, J. Optical Biosensors. Essays Biochem. 2016, 60 (1), 91-100. https://doi.org/10.1042/EBC20150010.

(39) Takemura K. Surface plasmon resonance (SPR)-and localized SPR (LSPR)-based virus sensing systems: Optical vibration of nano-and micro-metallic materials for the development of nextgeneration virus detection technology. Biosensors. 2021;11(8):250.

(40) Mora, M. F.; Wehmeyer, J. L.; Synowicki, R.; Garcia, C. D. Investigating Protein Adsorption via Spectroscopic Ellipsometry. Biol. Interact. Mater. Surf. 2009, 19-41. https://doi.org/10.1007/9780-387-98161-1_2.

(41) Dixon, M. C. Quartz Crystal Microbalance with Dissipation Monitoring: Enabling Real-Time Characterization of Biological Materials and Their Interactions. J. Biomol. Tech. JBT 2008, 19 (3), 151-158.

(42) Pimková, K.; Bocková, M.; Hegnerová, K.; Suttnar, J.; Cermák, J.; Homola, J.; Dyr, J. E. Surface Plasmon Resonance Biosensor for the Detection of VEGFR-1--a Protein Marker of Myelodysplastic Syndromes. Anal. Bioanal. Chem. 2012, 402 (1), 381-387. https://doi.org/10.1007/s00216-0115395-3.

(43) Man, Y.; Liang, G.; Li, A.; Pan, L. Recent Advances in Mycotoxin Determination for Food Monitoring via Microchip. Toxins 2017, 9 (10). https://doi.org/10.3390/toxins9100324.

(44) Fernández, F.; Hegnerová, K.; Piliarik, M.; Sanchez-Baeza, F.; Homola, J.; Marco, M.-P. A LabelFree and Portable Multichannel Surface Plasmon Resonance Immunosensor for on Site Analysis of Antibiotics in Milk Samples. Biosens. Bioelectron. 2010, 26 (4), 1231-1238.

https://doi.org/10.1016/j.bios.2010.06.012.

(45) Choi, Y.; Kwak, H.; Hong, S. Quantification of Arsenic(III) in Aqueous Media Using a Novel Hybrid Platform Comprised of Radially Porous Silica Particles and a Gold Thin Film. Anal. Methods 2014, 6 (17), 7054-7061. https://doi.org/10.1039/C4AY01297F.

(46) Krpetić, Z.; Guerrini, L.; Larmour, I. A.; Reglinski, J.; Faulds, K.; Graham, D. Importance of Nanoparticle Size in Colorimetric and SERS-Based Multimodal Trace Detection of $\mathrm{Ni}(\mathrm{II})$ lons with Functional Gold Nanoparticles. Small Weinh. Bergstr. Ger. 2012, 8 (5), 707-714. https://doi.org/10.1002/smll.201101980.

(47) Zhao, W.; Brook, M. A.; Li, Y. Design of Gold Nanoparticle-Based Colorimetric Biosensing Assays. Chembiochem Eur. J. Chem. Biol. 2008, 9 (15), 2363-2371.

https://doi.org/10.1002/cbic.200800282.

(48) Sepúlveda B, Angelomé PC, Lechuga LM, Liz-Marzán LM. LSPR-based nanobiosensors. Nano today. 2009 Jun 1;4(3):244-51.

(49) Liu, B.; Zhuang, J.; Wei, G. Recent Advances in the Design of Colorimetric Sensors for Environmental Monitoring. Environ. Sci. Nano 2020, 7 (8), 2195-2213. 
(50) Aldewachi, H.; Chalati, T.; N. Woodroofe, M.; Bricklebank, N.; Sharrack, B.; Gardiner, P. Gold Nanoparticle-Based Colorimetric Biosensors. Nanoscale 2018, 10 (1), 18-33. https://doi.org/10.1039/C7NR06367A.

(51) Liu, Y.; Zhang, L.; Wei, W.; Zhao, H.; Zhou, Z.; Zhang, Y.; Liu, S. Colorimetric Detection of Influenza A Virus Using Antibody-Functionalized Gold Nanoparticles. The Analyst 2015, 140 (12), 39893995. https://doi.org/10.1039/c5an00407a.

(52) Ku, T.-H.; Zhang, T.; Luo, H.; Yen, T. M.; Chen, P.-W.; Han, Y.; Lo, Y.-H. Nucleic Acid Aptamers: An Emerging Tool for Biotechnology and Biomedical Sensing. Sensors 2015, 15 (7), 16281-16313. https://doi.org/10.3390/s150716281.

(53) Doroszkowski, A. 6 - The Physical Chemistry of Dispersion. In Paint and Surface Coatings (Second Edition); Lambourne, R., Strivens, T. A., Eds.; Woodhead Publishing Series in Metals and Surface Engineering; Woodhead Publishing, 1999; pp 198-242. https://doi.org/10.1533/9781855737006.198.

(54) Sardar, R.; Funston, A. M.; Mulvaney, P.; Murray, R. W. Gold Nanoparticles: Past, Present, and Future. Langmuir 2009, 25 (24), 13840-13851. https://doi.org/10.1021/la9019475.

(55) Selegård, R.; Enander, K.; Aili, D. Generic Phosphatase Activity Detection Using Zinc Mediated Aggregation Modulation of Polypeptide-Modified Gold Nanoparticles. Nanoscale 2014, 6 (23), 14204-14212. https://doi.org/10.1039/C4NR02791D.

(56) Sato, K.; Hosokawa, K.; Maeda, M. Non-Cross-Linking Gold Nanoparticle Aggregation as a Detection Method for Single-Base Substitutions. Nucleic Acids Res. 2005, 33 (1), e4. https://doi.org/10.1093/nar/gni007.

(57) Sato, K.; Hosokawa, K.; Maeda, M. Rapid Aggregation of Gold Nanoparticles Induced by NonCross-Linking DNA Hybridization. J. Am. Chem. Soc. 2003, 125 (27), 8102-8103. https://doi.org/10.1021/ja034876s.

(58) Li; Rothberg, L. J. Label-Free Colorimetric Detection of Specific Sequences in Genomic DNA Amplified by the Polymerase Chain Reaction. J. Am. Chem. Soc. 2004, 126 (35), 10958-10961. https://doi.org/10.1021/ja048749n.

(59) Li, H.; Rothberg, L. Colorimetric Detection of DNA Sequences Based on Electrostatic Interactions with Unmodified Gold Nanoparticles. Proc. Natl. Acad. Sci. U. S. A. 2004, 101 (39), 14036-14039. https://doi.org/10.1073/pnas.0406115101.

(60) Thanh NT, Rosenzweig Z. Development of an aggregation-based immunoassay for anti-protein A using gold nanoparticles. Analytical chemistry. 2002 1;74(7):1624-8.

(61) Hone, D. C.; Haines, A. H.; Russell, D. A. Rapid, Quantitative Colorimetric Detection of a Lectin Using Mannose-Stabilized Gold Nanoparticles. Langmuir 2003, 19 (17), 7141-7144. https://doi.org/10.1021/la034358v.

(62) Wang, Z.; Lévy, R.; Fernig, D. G.; Brust, M. Kinase-Catalyzed Modification of Gold Nanoparticles: A New Approach to Colorimetric Kinase Activity Screening. J. Am. Chem. Soc. 2006, 128 (7), 22142215. https://doi.org/10.1021/ja058135y.

(63) Guarise, C.; Pasquato, L.; Filippis, V. D.; Scrimin, P. Gold Nanoparticles-Based Protease Assay. Proc. Natl. Acad. Sci. 2006, 103 (11), 3978-3982. https://doi.org/10.1073/pnas.0509372103.

(64) Chen, C.-K.; Huang, C.-C.; Chang, H.-T. Label-Free Colorimetric Detection of Picomolar Thrombin in Blood Plasma Using a Gold Nanoparticle-Based Assay. Biosens. Bioelectron. 2010, 25 (8), 19221927. https://doi.org/10.1016/j.bios.2010.01.005.

(65) Zhang, L.; Zhao, J.; Jiang, J.; Yu, R. Enzyme-Regulated Unmodified Gold Nanoparticle Aggregation: A Label Free Colorimetric Assay for Rapid and Sensitive Detection of Adenosine Deaminase Activity and Inhibition. Chem. Commun. 2012, 48 (89), 10996-10998. https://doi.org/10.1039/C2CC36240F. 
(66) Kim, Y. S.; Kim, J. H.; Kim, I. A.; Lee, S. J.; Jurng, J.; Gu, M. B. A Novel Colorimetric Aptasensor Using Gold Nanoparticle for a Highly Sensitive and Specific Detection of Oxytetracycline. Biosens. Bioelectron. 2010, 26 (4), 1644-1649. https://doi.org/10.1016/j.bios.2010.08.046.

(67) Alsager, O. A.; Kumar, S.; Zhu, B.; Travas-Sejdic, J.; McNatty, K. P.; Hodgkiss, J. M. Ultrasensitive Colorimetric Detection of 17ß-Estradiol: The Effect of Shortening DNA Aptamer Sequences. Anal. Chem. 2015, 87 (8), 4201-4209. https://doi.org/10.1021/acs.analchem.5b00335.

(68) Colorimetric and ratiometric aggregation assay for streptomycin using gold nanoparticles and a new and highly specific aptamer | SpringerLink https://link.springer.com/article/10.1007\%2Fs00604-016-1798-3 (accessed 2018 -04 -26).

(69) Ellington, A. D.; Szostak, J. W. In Vitro Selection of RNA Molecules That Bind Specific Ligands. Nature 1990, 346 (6287), 818-822. https://doi.org/10.1038/346818a0.

(70) Matsuda Y, Nonaka Y, Futakawa S, Imai H, Akita K, Nishihata T, Fujiwara M, Ali Y, Bhisitkul RB, Nakamura Y. Anti-angiogenic and anti-scarring dual action of an anti-fibroblast growth factor 2 aptamer in animal models of retinal disease. Molecular Therapy-Nucleic Acids. 2019 6;17:819-28.

(71) Roderick, W. R. The Double Helix. A Personal Account of the Discovery of the Structure of DNA (Watson, James D.). J. Chem. Educ. 1968, 45 (9), 623. https://doi.org/10.1021/ed045p623.2.

(72) O'Reilly RK, Turberfield AJ, Wilks TR. The evolution of DNA-templated synthesis as a tool for materials discovery. Accounts of Chemical Research. 2017 17;50(10):2496-509.

(73) Keefe, A. D.; Pai, S.; Ellington, A. Aptamers as Therapeutics. Nat. Rev. Drug Discov. 2010, 9 (7), 537-550. https://doi.org/10.1038/nrd3141.

(74) Stoltenburg, R.; Nikolaus, N.; Strehlitz, B. Capture-SELEX: Selection of DNA Aptamers for Aminoglycoside Antibiotics. J. Anal. Methods Chem. 2012, 2012, e415697. https://doi.org/10.1155/2012/415697.

(75) Lee, E.-S.; Kim, E.-J.; Park, T.-K.; Bae, D.-W.; Cha, S.-S.; Kim, T.-W.; Kim, Y.-P. Gold NanoparticleAssisted SELEX as a Visual Monitoring Platform for the Development of Small Molecule-Binding DNA Aptasensors. Biosens. Bioelectron. 2021, 191, 113468.

https://doi.org/10.1016/j.bios.2021.113468.

(76) Gu, H.; Duan, N.; Wu, S.; Hao, L.; Xia, Y.; Ma, X.; Wang, Z. Graphene Oxide-Assisted NonImmobilized SELEX of Okdaic Acid Aptamer and the Analytical Application of Aptasensor. Sci. Rep. 2016, 6 (1), 21665. https://doi.org/10.1038/srep21665.

(77) Pan, W.; Clawson, G. A. The Shorter the Better: Reducing Fixed Primer Regions of Oligonucleotide Libraries for Aptamer Selection. Molecules 2009, 14 (4), 1353-1369.

https://doi.org/10.3390/molecules14041353.

(78) Stoltenburg, R.; Reinemann, C.; Strehlitz, B. SELEX-A (r)Evolutionary Method to Generate HighAffinity Nucleic Acid Ligands. Biomol. Eng. 2007, 24 (4), 381-403.

https://doi.org/10.1016/j.bioeng.2007.06.001.

(79) Selection and characterization of DNA aptamer against glucagon receptor by cell-SELEX | Scientific Reports https://www.nature.com/articles/s41598-017-05840-w (accessed 2021 -09 16).

(80) Amano R, Furukawa T, Sakamoto T. ITC measurement for high-affinity aptamers binding to their target proteins. InMicrocalorimetry of biological molecules 2019 (pp. 119-128). Humana Press, New York, NY.

(81) Lou, X.; Egli, M.; Yang, X. Determining Functional Aptamer-Protein Interaction by Biolayer Interferometry. Curr. Protoc. Nucleic Acid Chem. 2016, 67, 7.25.1-7.25.15. https://doi.org/10.1002/cpnc.18.

(82) Wang, T.; Chen, C.; Larcher, L. M.; Barrero, R. A.; Veedu, R. N. Three Decades of Nucleic Acid Aptamer Technologies: Lessons Learned, Progress and Opportunities on Aptamer Development. Biotechnol. Adv. 2019, 37 (1), 28-50. https://doi.org/10.1016/j.biotechadv.2018.11.001. 
(83) Jayasena, S. D. Aptamers: An Emerging Class of Molecules That Rival Antibodies in Diagnostics. Clin. Chem. 1999, 45 (9), 1628-1650.

(84) Le, A. T. H.; Krylova, S. M.; Kanoatov, M.; Desai, S.; Krylov, S. N. Ideal-Filter Capillary Electrophoresis (IFCE) Facilitates the One-Step Selection of Aptamers. Angew. Chem. Int. Ed. 2019, 58 (9), 2739-2743. https://doi.org/10.1002/anie.201812974.

(85) Zhong, W.; Pu, Y.; Tan, W.; Liu, J.; Liao, J.; Liu, B.; Chen, K.; Yu, B.; Hu, Y.; Deng, Y.; Zhang, J.; Liu, H. Identification and Application of an Aptamer Targeting Papillary Thyroid Carcinoma Using TissueSELEX. Anal. Chem. 2019, 91 (13), 8289-8297. https://doi.org/10.1021/acs.analchem.9b01000.

(86) Cheng, C.; Chen, Y. H.; Lennox, K. A.; Behlke, M. A.; Davidson, B. L. In Vivo SELEX for Identification of Brain-Penetrating Aptamers. Mol. Ther. Nucleic Acids 2013, 2, e67. https://doi.org/10.1038/mtna.2012.59.

(87) Souza, A. G.; Marangoni, K.; Fujimura, P. T.; Alves, P. T.; Silva, M. J.; Bastos, V. A. F.; Goulart, L. R.; Goulart, V. A. 3D Cell-SELEX: Development of RNA Aptamers as Molecular Probes for PC-3 Tumor Cell Line. Exp. Cell Res. 2016, 341 (2), 147-156. https://doi.org/10.1016/j.yexcr.2016.01.015.

(88) Song, K.-M.; Lee, S.; Ban, C. Aptamers and Their Biological Applications. Sensors 2012, 12 (1), 612-631. https://doi.org/10.3390/s120100612.

(89) Kohlberger, M.; Gadermaier, G. SELEX: Critical Factors and Optimization Strategies for Successful Aptamer Selection. Biotechnol. Appl. Biochem. n/a (n/a). https://doi.org/10.1002/bab.2244.

(90) Cho, E. J.; Lee, J.-W.; Ellington, A. D. Applications of Aptamers as Sensors. Annu. Rev. Anal. Chem. 2009, 2 (1), 241-264. https://doi.org/10.1146/annurev.anchem.1.031207.112851.

(91) Nelson, E. M.; Rothberg, L. J. Kinetics and Mechanism of Single-Stranded DNA Adsorption onto Citrate-Stabilized Gold Nanoparticles in Colloidal Solution. Langmuir 2011, 27 (5), 1770-1777. https://doi.org/10.1021/la102613f.

(92) Koo, K. M.; Sina, A. A. I.; Carrascosa, L. G.; Shiddiky, M. J. A.; Trau, M. DNA-Bare Gold Affinity Interactions: Mechanism and Applications in Biosensing. Anal. Methods 2015, 7 (17), 7042-7054. https://doi.org/10.1039/C5AY01479D.

(93) Kimura-Suda, H.; Petrovykh, D. Y.; Tarlov, M. J.; Whitman, L. J. Base-Dependent Competitive Adsorption of Single-Stranded DNA on Gold. J. Am. Chem. Soc. 2003, 125 (30), 9014-9015. https://doi.org/10.1021/ja035756n.

(94) He H, Dai J, Duan Z, Zheng B, Meng Y, Guo Y, Xiao D. Unusual sequence length-dependent gold nanoparticles aggregation of the ssDNA sticky end and its application for enzyme-free and signal amplified colorimetric DNA detection. Scientific reports. 2016 1;6(1):1-7.

(95) Mao, Y.; Fan, T.; Gysbers, R.; Tan, Y.; Liu, F.; Lin, S.; Jiang, Y. A Simple and Sensitive Aptasensor for Colorimetric Detection of Adenosine Triphosphate Based on Unmodified Gold Nanoparticles. Talanta 2017, 168, 279-285. https://doi.org/10.1016/j.talanta.2017.03.014.

(96) Huo, Y.; Qi, L.; Lv, X.-J.; Lai, T.; Zhang, J.; Zhang, Z.-Q. A Sensitive Aptasensor for Colorimetric Detection of Adenosine Triphosphate Based on the Protective Effect of ATP-Aptamer Complexes on Unmodified Gold Nanoparticles. Biosens. Bioelectron. 2016, 78, 315-320.

https://doi.org/10.1016/j.bios.2015.11.043.

(97) Derbyshire, N.; White, S. J.; Bunka, D. H. J.; Song, L.; Stead, S.; Tarbin, J.; Sharman, M.; Zhou, D.; Stockley, P. G. Toggled RNA Aptamers Against Aminoglycosides Allowing Facile Detection of Antibiotics Using Gold Nanoparticle Assays. Anal. Chem. 2012, 84 (15), 6595-6602. https://doi.org/10.1021/ac300815c.

(98) Song KM, Jeong E, Jeon W, Cho M, Ban C. Aptasensor for ampicillin using gold nanoparticle based dual fluorescence-colorimetric methods. Analytical and bioanalytical chemistry.

2012;402(6):2153-61. 
(99) Shayesteh, O. H.; Ghavami, R. Two Colorimetric Ampicillin Sensing Schemes Based on the Interaction of Aptamers with Gold Nanoparticles. Mikrochim. Acta 2019, 186 (7), 485. https://doi.org/10.1007/s00604-019-3524-4.

(100) Sharma, T. K.; Ramanathan, R.; Weerathunge, P.; Mohammadtaheri, M.; Daima, H. K.; Shukla, R.; Bansal, V. Aptamer-Mediated "turn-off/Turn-on" Nanozyme Activity of Gold Nanoparticles for Kanamycin Detection. Chem. Commun. Camb. Engl. 2014, 50 (100), 15856-15859. https://doi.org/10.1039/c4cc07275h.

(101) Niu, S.; Lv, Z.; Liu, J.; Bai, W.; Yang, S.; Chen, A. Colorimetric Aptasensor Using Unmodified Gold Nanoparticles for Homogeneous Multiplex Detection. PLOS ONE 2014, 9 (10), e109263. https://doi.org/10.1371/journal.pone.0109263.

(102) Ha, N.-R.; Jung, I.-P.; La, I.-J.; Jung, H.-S.; Yoon, M.-Y. Ultra-Sensitive Detection of Kanamycin for Food Safety Using a Reduced Graphene Oxide-Based Fluorescent Aptasensor. Sci. Rep. 2017, 7 (1), 40305. https://doi.org/10.1038/srep40305.

(103) Wang, T.; Chen, L.; Chikkanna, A.; Chen, S.; Brusius, I.; Sbuh, N.; Veedu, R. N. Development of Nucleic Acid Aptamer-Based Lateral Flow Assays: A Robust Platform for Cost-Effective Point-ofCare Diagnosis. Theranostics 2021, 11 (11), 5174-5196. https://doi.org/10.7150/thno.56471.

(104) Zhou, N.; Zhang, J.; Tian, Y. Aptamer-Based Spectrophotometric Detection of Kanamycin in Milk. Anal. Methods 2014, 6 (5), 1569-1574. https://doi.org/10.1039/C3AY41816B.

(105) Abedalwafa, M. A.; Tang, Z.; Qiao, Y.; Mei, Q.; Yang, G.; Li, Y.; Wang, L. An Aptasensor Strip-Based Colorimetric Determination Method for Kanamycin Using Cellulose Acetate Nanofibers Decorated DNA-Gold Nanoparticle Bioconjugates. Mikrochim. Acta 2020, 187 (6), 360. https://doi.org/10.1007/s00604-020-04348-x.

(106) A polyA aptamer-based label-free colorimetric biosensor for the detection of kanamycin in human serum - Analytical Methods (RSC Publishing) https://pubs.rsc.org/en/content/articlelanding/2020/ay/d0ay00326c (accessed 2021 -09 -16).

(107) Kolya, H.; Hashitsume, K.; Kang, C.-W. Recent Advances in Colorimetric Detection of Arsenic Using Metal-Based Nanoparticles. Toxics 2021, 9 (6), 143. https://doi.org/10.3390/toxics9060143.

(108) Li, L.; Li, B.; Qi, Y.; Jin, Y. Label-Free Aptamer-Based Colorimetric Detection of Mercury lons in Aqueous Media Using Unmodified Gold Nanoparticles as Colorimetric Probe. Anal. Bioanal. Chem. 2009, 393 (8), 2051-2057. https://doi.org/10.1007/s00216-009-2640-0.

(109) Zhang, F.; Huang, P.-J. J.; Liu, J. Sensing Adenosine and ATP by Aptamers and Gold Nanoparticles: Opposite Trends of Color Change from Domination of Target Adsorption Instead of Aptamer Binding. ACS Sens. 2020, 5 (9), 2885-2893. https://doi.org/10.1021/acssensors.0c01169.

(110) Hu Y, Huang Z, Liu B, Liu J. Hg (II) adsorption on gold nanoparticles dominates DNA-based labelfree colorimetric sensing. ACS Applied Nano Materials. 2021 8;4(2):1377-84.

(111) Zong, C.; Zhang, Z.; Liu, B.; Liu, J. Adsorption of Arsenite on Gold Nanoparticles Studied with DNA Oligonucleotide Probes. Langmuir 2019, 35 (22), 7304-7311. https://doi.org/10.1021/acs.langmuir.9b01161.

(112) Liu, X.; He, F.; Zhang, F.; Zhang, Z.; Huang, Z.; Liu, J. Dopamine and Melamine Binding to Gold Nanoparticles Dominates Their Aptamer-Based Label-Free Colorimetric Sensing. Anal. Chem. 2020, 92 (13), 9370-9378. https://doi.org/10.1021/acs.analchem.0c01773.

(113) Zhou, J.; Li, Y.; Wang, W.; Lu, Z.; Han, H.; Liu, J. Kanamycin Adsorption on Gold Nanoparticles Dominates Its Label-Free Colorimetric Sensing with Its Aptamer. Langmuir 2020, 36 (39), 1149011498. https://doi.org/10.1021/acs.langmuir.0c01786.

(114) Tao, X.; He, F.; Liu, X.; Zhang, F.; Wang, X.; Peng, Y.; Liu, J. Detection of Chloramphenicol with an Aptamer-Based Colorimetric Assay: Critical Evaluation of Specific and Unspecific Binding of Analyte Molecules. Mikrochim. Acta 2020, 187 (12), 668. https://doi.org/10.1007/s00604-02004644-6. 
(115) Zhang F, Liu J. Label-Free Colorimetric Biosensors Based on Aptamers and Gold Nanoparticles: A Critical Review. Analysis \& Sensing. 2021;1(1):30-43.

(116) Zhang, F.; Wang, S.; Liu, J. Gold Nanoparticles Adsorb DNA and Aptamer Probes Too Strongly and a Comparison with Graphene Oxide for Biosensing. Anal. Chem. 2019, 91 (22), 14743-14750. https://doi.org/10.1021/acs.analchem.9b04142.

(117) Aptamer-Based Colorimetric Probe for Cocaine | Journal of the American Chemical Society https://pubs.acs.org/doi/abs/10.1021/ja0259483 (accessed 2021 -09-04).

(118) Yarbakht, M.; Nikkhah, M. Unmodified Gold Nanoparticles as a Colorimetric Probe for Visual Methamphetamine Detection. J. Exp. Nanosci. 2016, 11 (7), 593-601. https://doi.org/10.1080/17458080.2015.1100333.

(119) Shi, Q.; Shi, Y.; Pan, Y.; Yue, Z.; Zhang, H.; Yi, C. Colorimetric and Bare Eye Determination of Urinary Methylamphetamine Based on the Use of Aptamers and the Salt-Induced Aggregation of Unmodified Gold Nanoparticles. Microchim. Acta 2015, 182 (3-4), 505-511. https://doi.org/10.1007/s00604-014-1349-8.

(120) Mao, K.; Yang, Z.; Li, J.; Zhou, X.; Li, X.; Hu, J. A Novel Colorimetric Biosensor Based on NonAggregated Au@Ag Core-Shell Nanoparticles for Methamphetamine and Cocaine Detection. Talanta 2017, 175, 338-346. https://doi.org/10.1016/j.talanta.2017.07.011.

(121) Sanli, S.; Moulahoum, H.; Ghorbanizamani, F.; Celik, E. G.; Timur, S. Ultrasensitive CovalentlyLinked Aptasensor for Cocaine Detection Based on Electrolytes-Induced Repulsion/Attraction of Colloids. Biomed. Microdevices 2020, 22 (3), 51. https://doi.org/10.1007/s10544-020-00507-2.

(122) Nie, J.; Zhang, D.-W.; Tie, C.; Zhou, Y.-L.; Zhang, X.-X. A Label-Free DNA Hairpin Biosensor for Colorimetric Detection of Target with Suitable Functional DNA Partners. Biosens. Bioelectron. 2013, 49, 236-242. https://doi.org/10.1016/j.bios.2013.05.020.

(123) Lodha, A.; Pandya, A.; G. Sutariya, P.; K. Menon, S. A Smart and Rapid Colorimetric Method for the Detection of Codeine Sulphate, Using Unmodified Gold Nanoprobe. RSC Adv. 2014, 4 (92), 50443-50448. https://doi.org/10.1039/C4RA06269H.

(124) Mao, K.; Yang, Z.; Du, P.; Xu, Z.; Wang, Z.; Li, X. G-Quadruplex-Hemin DNAzyme Molecular Beacon Probe for the Detection of Methamphetamine. RSC Adv. 2016, 6 (67), 62754-62759. https://doi.org/10.1039/C6RA04912E.

(125) Matulakul, P.; Vongpramate, D.; Kulchat, S.; Chompoosor, A.; Thanan, R.; Sithithaworn, P.; Sakonsinsiri, C.; Puangmali, T. Development of Low-Cost AuNP-Based Aptasensors with Truncated Aptamer for Highly Sensitive Detection of 8-Oxo-DG in Urine. ACS Omega 2020, 5 (28), 1742317430. https://doi.org/10.1021/acsomega.0c01834.

(126) Ruppert, C.; Phogat, N.; Laufer, S.; Kohl, M.; Deigner, H.-P. A Smartphone Readout System for Gold Nanoparticle-Based Lateral Flow Assays: Application to Monitoring of Digoxigenin. Mikrochim. Acta 2019, 186 (2), 119. https://doi.org/10.1007/s00604-018-3195-6.

(127) Ramalingam, S.; Collier, C. M.; Singh, A. A Paper-Based Colorimetric Aptasensor for the Detection of Gentamicin. Biosensors 2021, 11 (2), 29. https://doi.org/10.3390/bios11020029.

(128) Makuła, P.; Pacia, M.; Macyk, W. How To Correctly Determine the Band Gap Energy of Modified Semiconductor Photocatalysts Based on UV-Vis Spectra. J. Phys. Chem. Lett. 2018, 9 (23), 68146817. https://doi.org/10.1021/acs.jpclett.8b02892.

(129) Mäntele, W.; Deniz, E. UV-VIS Absorption Spectroscopy: Lambert-Beer Reloaded. Spectrochim. Acta. A. Mol. Biomol. Spectrosc. 2017, 173, 965-968. https://doi.org/10.1016/j.saa.2016.09.037.

(130) Iarossi, M.; Schiattarella, C.; Rea, I.; De Stefano, L.; Fittipaldi, R.; Vecchione, A.; Velotta, R.; Ventura, B. D. Colorimetric Immunosensor by Aggregation of Photochemically Functionalized Gold Nanoparticles. ACS Omega 2018, 3 (4), 3805-3812. https://doi.org/10.1021/acsomega.8b00265. 
(131) Jongjinakool, S.; Palasak, K.; Bousod, N.; Teepoo, S. Gold Nanoparticles-Based Colorimetric Sensor for Cysteine Detection. Energy Procedia 2014, 56, 10-18.

https://doi.org/10.1016/j.egypro.2014.07.126.

(132) Nilam, M.; Hennig, A.; Nau, W. M.; Assaf, K. I. Gold Nanoparticle Aggregation Enables Colorimetric Sensing Assays for Enzymatic Decarboxylation. Anal. Methods 2017, 9 (19), 27842787. https://doi.org/10.1039/C7AY00642J.

(133) Mondal, B.; Ramlal, S.; Lavu, P. S.; N, B.; Kingston, J. Highly Sensitive Colorimetric Biosensor for Staphylococcal Enterotoxin B by a Label-Free Aptamer and Gold Nanoparticles. Front. Microbiol. 2018, 0. https://doi.org/10.3389/fmicb.2018.00179.

(134) Sun L, Jin H, Zhao X, Liu Z, Guan Y, Yang Z, Zhang L, Zhang L. Unfolding and Conformational Variations of Thrombin-Binding DNA Aptamers: Synthesis, Circular Dichroism and Molecular Dynamics Simulations. ChemMedChem. 2014; 9(5):9931001.

(135) Pohl, F. M.; Jovin, T. M. Salt-Induced Co-Operative Conformational Change of a Synthetic DNA: Equilibrium and Kinetic Studies with Poly(DG-DC). J. Mol. Biol. 1972, 67 (3), 375-396. https://doi.org/10.1016/0022-2836(72)90457-3.

(136) Ivanov, V. I.; Minyat, E. E. The Transitions between Left- and Right-Handed Forms of Poly(DG-DC). Nucleic Acids Res. 1981, 9 (18), 4783-4798.

(137) Vorlícková, M. Conformational Transitions of Alternating Purine-Pyrimidine DNAs in Perchlorate Ethanol Solutions. Biophys. J. 1995, 69 (5), 2033-2043.

(138) Hardin, C. C.; Zarling, D. A.; Puglisi, J. D.; Trulson, M. O.; Davis, P. W.; Tinoco, I. Stabilization of ZRNA by Chemical Bromination and Its Recognition by Anti-Z-DNA Antibodies. Biochemistry 1987, 26 (16), 5191-5199. https://doi.org/10.1021/bi00390a044.

(139) Alternate rRNA secondary structures as regulators of translation | Nature Structural \& Molecular Biology https://www.nature.com/articles/nsmb.1962?message-global=remove\&annotation=1 (accessed $2021-08-03$ ).

(140) Vorlíčková, M.; Kejnovská, I.; Bednářová, K.; Renčiuk, D.; Kypr, J. Circular Dichroism Spectroscopy of DNA: From Duplexes to Quadruplexes. Chirality 2012, 24 (9), 691-698. https://doi.org/10.1002/chir.22064.

(141) Xi, H.; Juhas, M.; Zhang, Y. G-Quadruplex Based Biosensor: A Potential Tool for SARS-CoV-2 Detection. Biosens. Bioelectron. 2020, 167, 112494. https://doi.org/10.1016/j.bios.2020.112494.

(142) Shahsavar, K.; Hosseini, M.; Shokri, E.; Xu, G. New Insight into G-Quadruplexes; Diagnosis Application in Cancer. Anal. Biochem. 2021, 620, 114149. https://doi.org/10.1016/j.ab.2021.114149.

(143) Bhattacharjee, S. DLS and Zeta Potential - What They Are and What They Are Not? J. Controlled Release 2016, 235, 337-351. https://doi.org/10.1016/j.jconrel.2016.06.017.

(144) Wang W, Ding X, He M, Wang J, Lou X. Kinetic adsorption profile and conformation evolution at the DNA-gold nanoparticle interface probed by dynamic light scattering. Analytical chemistry. 2014 21;86(20):10186-92.

(145) Alsager, O. A.; Kumar, S.; Zhu, B.; Travas-Sejdic, J.; McNatty, K. P.; Hodgkiss, J. M. Ultrasensitive Colorimetric Detection of $17 \beta$-Estradiol: The Effect of Shortening DNA Aptamer Sequences. Anal. Chem. 2015, 87 (8), 4201-4209. https://doi.org/10.1021/acs.analchem.5b00335.

(146) Hakimian, F.; Ghourchian, H.; Hashemi, A. sadat; Arastoo, M. R.; Behnam Rad, M. Ultrasensitive Optical Biosensor for Detection of MiRNA-155 Using Positively Charged Au Nanoparticles. Sci. Rep. 2018, 8 (1), 2943. https://doi.org/10.1038/s41598-018-20229-z.

(147) Borse, V.; Konwar, A. N. Synthesis and Characterization of Gold Nanoparticles as a Sensing Tool for the Lateral Flow Immunoassay Development. Sens. Int. 2020, 1, 100051. 
(148) Raja DA, Munir F, Shah MR, Bhanger MI, Malik MI. Colorimetric sensing of cephradine through polypropylene glycol functionalized gold nanoparticles. Royal Society open science. 2021 26;8(5):210185.

(149) Kaur, T.; Tavakoli, N.; Slavcev, R.; Wettig, S. Calorimetric Investigations of Non-Viral DNA Transfection Systems; IntechOpen, 2011.

(150) Chaires, J. B. Calorimetry and Thermodynamics in Drug Design. Annu. Rev. Biophys. 2008, 37 (1), 135-151. https://doi.org/10.1146/annurev.biophys.36.040306.132812.

(151) Velázquez-Campoy, A.; Ohtaka, H.; Nezami, A.; Muzammil, S.; Freire, E. Isothermal Titration Calorimetry. Curr. Protoc. Cell Biol. 2004, 23 (1), 17.8.1-17.8.24. https://doi.org/10.1002/0471143030.cb1708s23.

(152) Cordeiro, M.; Ferreira Carlos, F.; Pedrosa, P.; Lopez, A.; Baptista, P. V. Gold Nanoparticles for Diagnostics: Advances towards Points of Care. Diagnostics 2016, 6 (4).

(153) St John, A.; Price, C. The March of Technology through the Clinical Laboratory and Beyond. Clin. Biochem. Rev. 2014, 35 (3), 139-141.

(154) Srinivasan, B.; Tung, S. Development and Applications of Portable Biosensors. J. Lab. Autom. 2015, 20 (4), 365-389. https://doi.org/10.1177/2211068215581349.

(155) Xia, F.; Zuo, X.; Yang, R.; Xiao, Y.; Kang, D.; Vallée-Bélisle, A.; Gong, X.; Yuen, J. D.; Hsu, B. B. Y.; Heeger, A. J.; Plaxco, K. W. Colorimetric Detection of DNA, Small Molecules, Proteins, and Ions Using Unmodified Gold Nanoparticles and Conjugated Polyelectrolytes. Proc. Natl. Acad. Sci. U. S. A. 2010, 107 (24), 10837-10841.

(156) Liu, G.; Lu, M.; Huang, X.; Li, T.; Xu, D. Application of Gold-Nanoparticle Colorimetric Sensing to Rapid Food Safety Screening. Sensors 2018, 18 (12), 4166. https://doi.org/10.3390/s18124166.

(157) Zhang, J.; Wang, L.; Pan, D.; Song, S.; Boey, F. Y. C.; Zhang, H.; Fan, C. Visual Cocaine Detection with Gold Nanoparticles and Rationally Engineered Aptamer Structures. Small Weinh. Bergstr. Ger. 2008, 4 (8), 1196-1200.

(158) Yang, C.; Wang, Y.; Marty, J.-L.; Yang, X. Aptamer-Based Colorimetric Biosensing of Ochratoxin A Using Unmodified Gold Nanoparticles Indicator. Biosens. Bioelectron. 2011, 26 (5), 2724-2727. https://doi.org/10.1016/j.bios.2010.09.032.

(159) O'Sullivan, C. K. Aptasensors - the Future of Biosensing? Anal. Bioanal. Chem. 2002, 372 (1), 4448.

(160) Kerwin, S. M. Nucleic Acids: Structures, Properties, and Functions By Victor A. Bloomfield, Donald M. Crothers, and Ignacio Tinoco, Jr., with Contributions from John E. Hearst, David E. Wemmer, Peter A. Kollman, and Douglas H. Turner. University Science Books, Sausalito, CA. 2000. Ix 794 Pp. $17 \times 25$ Cm. ISBN 0-935702-49-0. \$85.00. J. Med. Chem. 2000, 43 (24), 4721-4722.

(161) Li, H.; Rothberg, L. Colorimetric Detection of DNA Sequences Based on Electrostatic Interactions with Unmodified Gold Nanoparticles. Proc. Natl. Acad. Sci. U. S. A. 2004, 101 (39), 14036-14039.

(162) Surface Science of DNA Adsorption onto Citrate-Capped Gold Nanoparticles | Langmuir

(163) Findenegg, G. H. J. N. Israelachvili: Intermolecular and Surface Forces (With Applications to Colloidal and Biological Systems). Academic Press, London, Orlando, San Diego, New York, Toronto, Montreal, Sydney, Tokyo 1985. 296 Seiten, Preis: \$ 65.00. Berichte Bunsenges. Für Phys. Chem. 1986, 90 (12), 1241-1242.

(164) Sandström, P.; Boncheva, M.; Åkerman, B. Nonspecific and Thiol-Specific Binding of DNA to Gold Nanoparticles. Langmuir 2003, 19 (18), 7537-7543.

(165) Zhang, X.; Servos, M. R.; Liu, J. Surface Science of DNA Adsorption onto Citrate-Capped Gold Nanoparticles. Langmuir 2012, 28 (8), 3896-3902.

(166) Demers, L. M.; Ostblom, M.; Zhang, H.; Jang, N.-H.; Liedberg, B.; Mirkin, C. A. Thermal Desorption Behavior and Binding Properties of DNA Bases and Nucleosides on Gold. J. Am. Chem. Soc. 2002, 124 (38), 11248-11249. 
(167) Ostblom, M.; Liedberg, B.; Demers, L. M.; Mirkin, C. A. On the Structure and Desorption Dynamics of DNA Bases Adsorbed on Gold: A Temperature-Programmed Study. J. Phys. Chem. B 2005, 109 (31), 15150-15160..

(168) Nelson, E. M.; Rothberg, L. J. Kinetics and Mechanism of Single-Stranded DNA Adsorption onto Citrate-Stabilized Gold Nanoparticles in Colloidal Solution. Langmuir 2011, 27 (5), 1770-1777. https://doi.org/10.1021/la102613f.

(169) Kwon, Y. S.; Ahmad Raston, N. H.; Gu, M. B. An Ultra-Sensitive Colorimetric Detection of Tetracyclines Using the Shortest Aptamer with Highly Enhanced Affinity. Chem. Commun. Camb. Engl. 2014, 50 (1), 40-42.

(170) Mfold web server for nucleic acid folding and hybridization prediction | Nucleic Acids Research | Oxford Academic

(171) Mirau, P. A.; Smith, J. E.; Chávez, J. L.; Hagen, J. A.; Kelley-Loughnane, N.; Naik, R. Structured DNA Aptamer Interactions with Gold Nanoparticles. Langmuir 2018, 34 (5), 2139-2146. https://doi.org/10.1021/acs.langmuir.7b02449.

(172) Lee, B. H.; Nguyen, V. T.; Gu, M. B. Highly Sensitive Detection of 25-HydroxyvitaminD3 by Using a Target-Induced Displacement of Aptamer. Biosens. Bioelectron. 2017, 88, 174-180. https://doi.org/10.1016/j.bios.2016.08.011.

(173) Liu, J.; Bai, W.; Niu, S.; Zhu, C.; Yang, S.; Chen, A. Highly Sensitive Colorimetric Detection of 17 $\beta-$ Estradiol Using Split DNA Aptamers Immobilized on Unmodified Gold Nanoparticles. Sci. Rep. 2014, 4 (1), 7571. https://doi.org/10.1038/srep07571.

(174) Alsager, O. A.; Alotaibi, K. M.; Alswieleh, A. M.; Alyamani, B. J. Colorimetric Aptasensor of Vitamin D3: A Novel Approach to Eliminate Residual Adhesion between Aptamers and Gold Nanoparticles. Sci. Rep. 2018, 8 (1), 12947. https://doi.org/10.1038/s41598-018-31221-y.

(175) Yarbakht, M.; Nikkhah, M. Unmodified Gold Nanoparticles as a Colorimetric Probe for Visual Methamphetamine Detection. J. Exp. Nanosci. 2016, 11 (7), 593-601.

(176) Oghli, A. H.; Alipour, E.; Asadzadeh, M. Development of a Novel Voltammetric Sensor for the Determination of Methamphetamine in Biological Samples on the Pretreated Pencil Graphite Electrode. RSC Adv. 2015, 5 (13), 9674-9682.

(177) Shi, Q.; Shi, Y.; Pan, Y.; Yue, Z.; Zhang, H.; Yi, C. Colorimetric and Bare Eye Determination of Urinary Methylamphetamine Based on the Use of Aptamers and the Salt-Induced Aggregation of Unmodified Gold Nanoparticles. Microchim. Acta 2015, 182 (3-4), 505-511. https://doi.org/10.1007/s00604-014-1349-8.

(178) Yarbakht, M.; Nikkhah, M. Unmodified Gold Nanoparticles as a Colorimetric Probe for Visual Methamphetamine Detection. J. Exp. Nanosci. 2016, 11 (7), 593-601.

(179) G-quadruplex aptamer targeting Protein A and its capability to detect Staphylococcus aureus demonstrated by ELONA | Scientific Reports

(180) Wemmer, D. E. Designed Sequence-Specific Minor Groove Ligands. Annu. Rev. Biophys. Biomol. Struct. 2000, 29 (1), 439-461. https://doi.org/10.1146/annurev.biophys.29.1.439.

(181) Chaires, J. B. A Thermodynamic Signature for Drug-DNA Binding Mode. Arch. Biochem. Biophys. 2006, 453 (1), 26-31.

(182) Velazquez-Campoy, A.; Todd, M. J.; Freire, E. HIV-1 Protease Inhibitors: Enthalpic versus Entropic Optimization of the Binding Affinity. Biochemistry 2000, 39 (9), 2201-2207.

(183) Velázquez Campoy, A.; Freire, E. ITC in the Post-Genomic Era...? Priceless. Biophys. Chem. 2005, $115(2), 115-124$.

(184) Kim, A.-R.; Choi, Y.; Kim, S.-H.; Moon, H.-S.; Ko, J.-H.; Yoon, M.-Y. Development of a Novel SsDNA Sequence for a Glycated Human Serum Albumin and Construction of a Simple Aptasensor System Based on Reduced Graphene Oxide (RGO). Biosensors 2020, 10 (10), 141. 
(185) Publishers, B. S. Combinational Chemistry \& High Throughput Screening; Bentham Science Publishers, 1999.

(186) Selection of single-stranded DNA molecules that bind and inhibit human thrombin | Nature

(187) Selection of Aptamers against Live Bacterial Cells | Analytical Chemistry

(188) In Vitro Selection of High-Affinity DNA Ligands for Human IgE Using Capillary Electrophoresis I Analytical Chemistry https://pubs.acs.org/doi/abs/10.1021/ac049857v (accessed 2021 -08 -14).

(189) Le, T. T.; Chumphukam, O.; Cass, A. E. G. Determination of Minimal Sequence for Binding of an Aptamer. A Comparison of Truncation and Hybridization Inhibition Methods. RSC Adv 2014, 4 (88), 47227-47233.

(190) Maio, G.; Enweronye, O.; Zumrut, H. E.; Batool, S.; Van, N.; Mallikaratchy, P. Systematic Optimization and Modification of a DNA Aptamer with 2'-O-Methyl RNA Analogues. ChemistrySelect 2017, 2 (7), 2335-2340.

(191) Wang, X.; Gao, X.; He, J.; Hu, X.; Li, Y.; Li, X.; Fan, L.; Yu, H.-Z. Systematic Truncating of Aptamers to Create High-Performance Graphene Oxide (GO)-Based Aptasensors for the Multiplex Detection of Mycotoxins. Analyst 2019, 144 (12), 3826-3835. https://doi.org/10.1039/C9AN00624A.

(192) Bottari, F.; Daems, E.; de Vries, A.-M.; Van Wielendaele, P.; Trashin, S.; Blust, R.; Sobott, F.; Madder, A.; Martins, J. C.; De Wael, K. Do Aptamers Always Bind? The Need for a Multifaceted Analytical Approach When Demonstrating Binding Affinity between Aptamer and Low Molecular Weight Compounds. J. Am. Chem. Soc. 2020, 142 (46), 19622-19630.

(193) Humphrey, S. P.; Williamson, R. T. A Review of Saliva: Normal Composition, Flow, and Function. J. Prosthet. Dent. 2001, 85 (2), 162-169. https://doi.org/10.1067/mpr.2001.113778.

(194) Punj, A. Secretions of Human Salivary Gland. In Salivary Glands - New Approaches in Diagnostics and Treatment; Adadan Güvenç, I., Ed.; IntechOpen, 2019.

(195) Pfaffe, T.; Cooper-White, J.; Beyerlein, P.; Kostner, K.; Punyadeera, C. Diagnostic Potential of Saliva: Current State and Future Applications. Clin. Chem. 2011, 57 (5), 675-687.

(196) Kaufman. E; Ira. B; The Diagnostic Applications of Saliva- A Review - Lamster, 2002

(197) Schramm, W.; Smith, R. H.; Craig, P. A.; Kidwell, D. A. Drugs of Abuse in Saliva: A Review. J. Anal. Toxicol. 1992, 16 (1), 1-9.

(198) van den Elsen, S. H. J.; Oostenbrink, L. M.; Heysell, S. K.; Hira, D.; Touw, D. J.; Akkerman, O. W.; Bolhuis, M. S.; Alffenaar, J.-W. C. Systematic Review of Salivary Versus Blood Concentrations of Antituberculosis Drugs and Their Potential for Salivary Therapeutic Drug Monitoring. Ther. Drug Monit. 2018, 40 (1), 17-37.

(199) Huestis, M. A.; Cone, E. J. Methamphetamine Disposition in Oral Fluid, Plasma, and Urine. Ann. N. Y. Acad. Sci. 2007, 1098, 104-121.

(200) Schepers, R. J. F.; Oyler, J. M.; Joseph, R. E., Jr; Cone, E. J.; Moolchan, E. T.; Huestis, M. A. Methamphetamine and Amphetamine Pharmacokinetics in Oral Fluid and Plasma after Controlled Oral Methamphetamine Administration to Human Volunteers. Clin. Chem. 2003, 49 (1), 121-132.

(201) Brun, E.; Sicard-Roselli, C. Could Nanoparticle Corona Characterization Help for Biological Consequence Prediction? Cancer Nanotechnol. 2014, 5 (1), 7.

(202) Mohamed, R.; Campbell, J.-L.; Cooper-White, J.; Dimeski, G.; Punyadeera, C. The Impact of Saliva Collection and Processing Methods on CRP, IgE, and Myoglobin Immunoassays. Clin. Transl. Med. 2012, 1, 19.

(203) Atkinson, K. R.; Lo, K. R.; Payne, S. R.; Mitchell, J. S.; Ingram, J. R. Rapid Saliva Processing Techniques for near Real-Time Analysis of Salivary Steroids and Protein. J. Clin. Lab. Anal. 2018, 22 (6), 395-402.

(204) Neyraud E, Prinz J, Dransfield E. NaCl and sugar release, salivation and taste during mastication of salted chewing gum. Physiology \& behavior. 2003 1;79(4-5):7317. 
(205) Loo, J. A.; Yan, W.; Ramachandran, P.; Wong, D. T. Comparative Human Salivary and Plasma Proteomes. J. Dent. Res. 2010, 89 (10), 1016-1023.

(206) Zhang, X.; Servos, M. R.; Liu, J. Surface Science of DNA Adsorption onto Citrate-Capped Gold Nanoparticles. Langmuir 2012, 28 (8), 3896-3902.

(207) Brown R. Drug and alcohol nursing. An Introduction to Community and Primary Health Care. 2020 30:339.

(208) Silber, B. Y.; Croft, R. J.; Downey, L. A.; Camfield, D. A.; Papafotiou, K.; Swann, P.; Stough, C. The Effect of d,I-Methamphetamine on Simulated Driving Performance. Psychopharmacology (Berl.) 2012, 219 (4), 1081-1087.

(209) Bosanquet, D.; MacDougall, H. G.; Rogers, S. J.; Starmer, G. A.; McKetin, R.; Blaszczynski, A.; McGregor, I. S. Driving on Ice: Impaired Driving Skills in Current Methamphetamine Users. Psychopharmacology (Berl.) 2013, 225 (1), 161-172.

(210) Ramaekers, J. G.; Kuypers, K. P. C.; Samyn, N. Stimulant Effects of 3,4 Methylenedioxymethamphetamine (MDMA) $75 \mathrm{Mg}$ and Methylphenidate $20 \mathrm{Mg}$ on Actual Driving during Intoxication and Withdrawal. Addict. Abingdon Engl. 2006, 101 (11), 1614-1621.

(211) Xiao, W.; Xiao, M.; Fu, Q.; Yu, S.; Shen, H.; Bian, H.; Tang, Y. A Portable Smart-Phone Readout Device for the Detection of Mercury Contamination Based on an Aptamer-Assay Nanosensor. Sensors 2016, 16 (11), $1871 .$. 